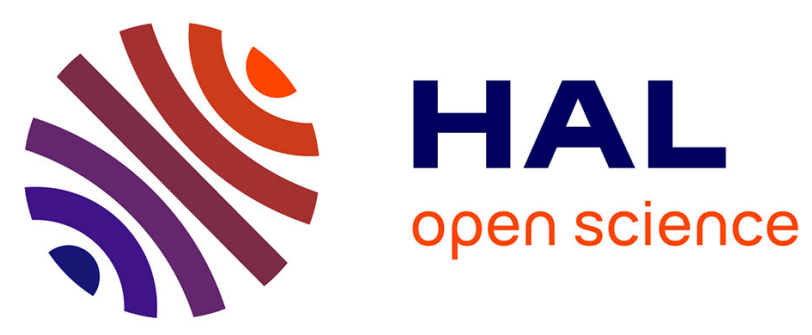

\title{
Measurements of visual binaries with PISCO2 at the Nice 76-cm refractor in 2011-2012
}

René Gili, Jean-Louis Prieur, Jean-pierre Rivet, Farrokh Vakili, Marco

Scardia, Luigi Pansecchi, Robert W. Argyle, Josefina F Ling, Luca Piccotti, Eric Aristidi, et al.

\section{To cite this version:}

René Gili, Jean-Louis Prieur, Jean-pierre Rivet, Farrokh Vakili, Marco Scardia, et al.. Measurements of visual binaries with PISCO2 at the Nice 76-cm refractor in 2011-2012. Astronomical Notes / Astronomische Nachrichten, 2021, 342 (6), pp.865-889. 10.1002/asna.202113985 . hal-03290780v2

\section{HAL Id: hal-03290780 \\ https://hal.science/hal-03290780v2}

Submitted on 26 Jun 2022

HAL is a multi-disciplinary open access archive for the deposit and dissemination of scientific research documents, whether they are published or not. The documents may come from teaching and research institutions in France or abroad, or from public or private research centers.
L'archive ouverte pluridisciplinaire HAL, est destinée au dépôt et à la diffusion de documents scientifiques de niveau recherche, publiés ou non, émanant des établissements d'enseignement et de recherche français ou étrangers, des laboratoires publics ou privés. 


\title{
Measurements of visual binaries with PISCO2 at the Nice 76- cm refractor in 2011-2012
}

\author{
R. Gili ${ }^{\dagger 1}$, J.-L. Prieur ${ }^{2,3, \star}$, J.-P. Rivet ${ }^{4}$ F. Vakili ${ }^{4}$, M. Scardia ${ }^{5,6}$, L. Pansecchi ${ }^{6}$, \\ R.W. Argyle ${ }^{7}$, J.F. Ling ${ }^{8,9}$ L. Piccotti ${ }^{8,9}$, E. Aristidi ${ }^{4}$, L. Koechlin ${ }^{2,3}$, D. Bonneau ${ }^{4}$, \\ L. Maccarini ${ }^{10}$, and $\mathbf{J}$. Serot ${ }^{11}$ \\ 1 Université Côte d'Azur, Observatoire de la Côte d'Azur, CNRS, Unité Mixte de Service Galilée, France \\ 2 Université de Toulouse - UPS-OMP - IRAP, Toulouse, France \\ 3 CNRS - IRAP, 14 avenue Edouard Belin, 31400 Toulouse, France \\ 4 Université Côte d'Azur, Observatoire de la Côte d'Azur, CNRS, Laboratoire Lagrange, France \\ 5 Université Côte d'Azur, Observatoire de la Côte d'Azur - C2PU, Nice, France \\ ${ }^{6}$ INAF - Osservatorio Astronomico di Brera, Via E. Bianchi 46, 23807 Merate, Italy \\ 7 Institute of Astronomy, Madingley Road, Cambridge, CB3 0HA, United Kingdom \\ 8 Observatorio Astronómico R.M. Aller, Avda das Ciencias s/n. Departamento de Matemática Aplicada, \\ Universidad de Santiago de Compostela, 15782, Spain \\ 9 Instituto de Matemáticas y Departamento de Matemática Aplicada, Facultade de Matemáticas, Univer- \\ sidad de Santiago de Compostela, 15782, Spain \\ 10 Desio, Monza and Brianza, Italy \\ 11 Institut Pascal - CNRS UMR 6602, Avenue B. Pascal 63178 Aubière, France
}

Received June 26, 2022; accepted

Key words Stars: binaries: close - binaries: visual — astrometry - photometry — techniques: speckle interferometry, Lucky imaging, direct vector autocorrelation — instruments: 76-cm refractor

We present relative astrometric and photometric measurements of visual binaries made in 2011-2012, with PISCO2 installed at the 76-cm refractor of Côte d'Azur Observatory in Nice (France). Our observing list contains orbital couples as well as binaries whose motion is still uncertain. Three different techniques have been used for obtaining those measurements: Lucky Imaging, Speckle Interferometry and the Direct Vector Autocorrelation method. From our observations of 3136 multiple stars, we obtained 3568 new measurements with angular separations in the range $0^{\prime \prime} .1-10^{\prime \prime}$ and an average accuracy of $0^{\prime \prime} .016$. The mean error on the position angles is $1^{\circ} .7$. Most of the position angles were determined without the usual $180^{\circ}$ ambiguity with the application of the direct vector autocorrelation technique and/or by inspection of the Lucky images or the long integration files. We managed to routinely monitor faint systems $\left(m_{V} \approx 9-11\right)$ with large magnitude difference (up to $\Delta m_{V} \approx 5$ ). We have thus been able to measure many systems containing red dwarf stars that had been poorly monitored since their discovery. We also measured the magnitude difference of the two components of 326 binaries with an estimated error of 0.2 mag. Except for a few objects that have been discussed, our measurements are in a good agreement with the ephemerides computed with published orbital elements, even for the binaries closer than the diffraction limit. Thanks to good seeing images and with the use of high-contrast numerical filters, we have also been able to obtain 196 measurements with an angular separation smaller than the diffraction limit of our instrumentation, and consistent with those obtained with larger telescopes. We also obtained new measurements of the 15th magnitude multiple system CON37, and discovered a faint new double star in its vicinity. Finally, we report 47 measurements of 28 new binaries found during our observations.

${ }^{\dagger}$ Deceased in October 2018

\section{Introduction}

This paper presents the results of speckle observations of visual binary stars made in 2011-2012 with the PISCO2 instrument (Pupil Interferometry Speckle camera and COronagraph, 2nd Version) installed at the

^ Corresponding author: jean-louis.prieur@irap.omp.eu 76-cm refractor telescope of the "Observatoire de la Côte d'Azur", (OCA) in Nice (France).

Built in 1887 , when it was the largest refractor in the world, this famous telescope has a free aperture diameter of $D=74 \mathrm{~cm}$ and a focal length of $17.89 \mathrm{~m}$. It is one of the largest refractors still in operation in 2012 in the world. The good quality of its optics makes Airy rings clearly visible when the seeing is good. And this happens very frequently, despite the vicinity to the city 
of Nice. It has mainly been devoted to binary observation since its construction.

PISCO2 is a speckle camera that was specially designed for this telescope and was used for the first time for those observations.

Our research program aims at obtaining high angular resolution measurements of binary stars with sensitive detectors, by using the good image quality of the site and the optics of the large Nice refractor, that often provides diffraction-limited images. The final purpose is to contribute to a better knowledge of the apparent relative motion of long-period binaries so that very accurate orbits could be determined in the future and fundamental parameters such as stellar masses could be inferred from them. The work presented here is the continuation of the program described in Gili \& Prieur (2012) and Gili et al. (2020), that will be referred as Paper I and PaperII, hereafter.

We describe PISCO2 and the instrumental setup in Sect. 2 and the conditions of our observations in Sect. 3. We introduce our calibration and data-reduction procedures in Sects. 4-5. We present and discuss our relative astrometric and photometric measurements in Sect. 6 . Then in Sect. 7 we compare our measurements with the ephemerides computed with published orbital elements, when available. We discuss the few cases of the largest residuals, and then focus on the measurements of binaries closer than the diffraction limit of our instrumentation. Finally in Sect. 8 we describe our observation of the 15th magnitude multiple system CON37, with new measurements and the discovery of a new binary.

\section{PISCO2}

PISCO2 is a simplified version of PISCO (see Prieur et al. (1998)), a multi-purpose focal instrument that was developed in 1993 for the 2-meter Bernard Lyot telescope (Pic du Midi Observatory, France). PISCO has been operated in 2004-2015 on a dedicated 1-meter telescope in Merate (Brera Observatory, Italy) (see e.g., Scardia et al., 2007, 2011) and since 2015 on the 1meter Epsilon telescope of C2PU (Center for Pedagogy in Earth and Universe Sciences) on the Calern site of OCA (Scardia et al., 2019). PISCO has been mainly used for investigations on visual binary stars with a few thousand measurements and a series of more than twenty publications on that field.

Like PISCO, PISCO2 is a focal instrument that provides high angular resolution images using speckle interferometry techniques that partly overcome the degradation caused by atmospheric turbulence (Labeyrie, 1970). PISCO 2 was entirely made at OCA between 2010 and 2012. It is described in detail in Gili et al. (2014).
Fitted with an external sensitive detector (see Figs. 1, 2), PISCO2 allows the acquisition of short exposure images that are processed in real time by our specially designed software. Two detectors are currently available, the Andor iXon DV885 and the Andor iXon DV897 cameras (see properties in Paper II, and in ANDOR, 2021). The observations presented here were obtained with the DV897 camera only. Two Risley prisms are used for correcting the atmospheric dispersion. Most optical settings, like focusing and Risley prism positioning, are remotely controlled.

The optical design of PISCO2 is shown in Fig. 2. It is similar to that of PISCO but the observing modes are reduced and are mainly limited to speckle observations and to the observation of visual binary stars in particular. Associated with an electronic detector, PISCO2 allows the acquisition of enlarged short exposure images exhibiting "speckles". Those images can then be processed as first suggested by Labeyrie (1970), to provide high-angular resolution information.

\section{Observations}

\subsection{Observing list}

Our list of targets is the same as in Paper I and Paper II, and was presented in Paper I. It basically includes all the visual binaries of the "Washington Double Star Catalog" (Mason et al., 2001, hereafter: WDS) for which new measurements are needed to improve their orbits, and that are accessible with our instrumentation. Among those, we particularly favoured the objects discovered by Paul Couteau (see Couteau, 1999, and Le Contel et al., 2001), since they are not often measured by other observers because most of them are rather faint and have a small separation. Indeed they are particularly well suited to the $76-\mathrm{cm}$ refractor and this site, since most of them were discovered in Nice.

The calendar of the observations made in 2011-2012 is reported in Table 1, with the distribution of the observations during those years and some comments. We indicate the epoch in the first column and the corresponding number of observations in Col. 2. We then give the night distribution of those observations during the month in Col. 3, the number of nights used for the observations in Col. 4, and some comments in Col. 5. In 2011-2012 we had to solve some problems, such as a dome shutter breakdown, a change of the DV897 eprom circuit, dome maintenance, and right ascension tracking problems. Finally the Risley prisms used for the atmospheric correction (see Sect. 2) were implemented to PISCO2 in October 2012.

The distribution of the apparent magnitudes $m_{V}$ of the primary components of the binaries observed in 2011-2012 is presented in Fig. 3a and the difference of magnitudes $\Delta m_{V}$ between the two components in 

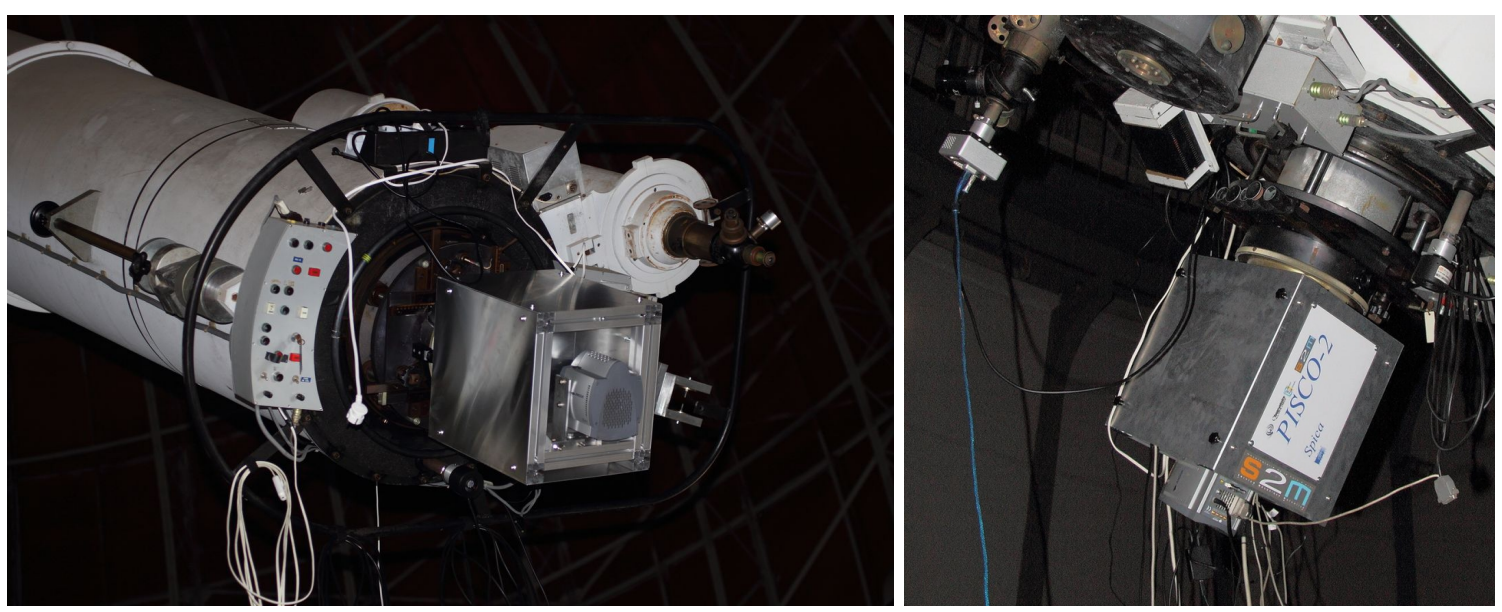

Fig. 1 PISCO2 with the ANDOR DV897 EMCCD camera at the focus of the 76-cm refractor.

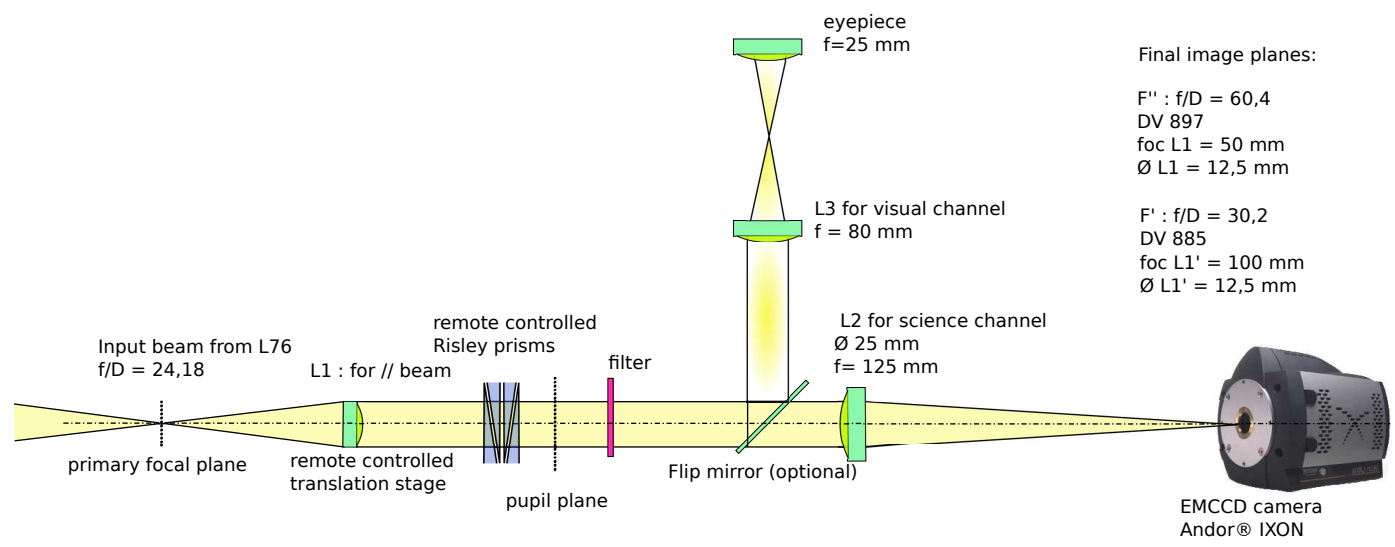

Fig. 2 PISCO2: optical layout, with the two configurations corresponding to the two available cameras that are fitted with the DV885 or the DV897 detectors. The data presented in this paper were obtained with the DV897 only.

Table 1 Calendar of the observations of double stars in 2011-2012.

\begin{tabular}{|c|c|c|c|c|}
\hline Month & Nobs. & Night distribution during the month & Nights & Comments \\
\hline 2011-01 & 155 & $=20+104+31$ & 3 & \\
\hline 2011-02 & 92 & $=64+28$ & 2 & \\
\hline 2011-03 & 360 & $=29+71+64+58+42+50+46$ & 7 & \\
\hline 2011-04 & 234 & $=54+38+50+31+48+13$ & 6 & \\
\hline 2011-05 & 166 & $=47+29+32+29+29$ & 5 & \\
\hline 2011-06 & 291 & $=24+14+15+47+52+37+8+50+52$ & 9 & \\
\hline 2011-07 & 459 & $=52+48+39+50+14+52+31+30+60+40+43$ & 11 & \\
\hline 2011-08 & 47 & & 1 & Shutter breakdown \\
\hline 2011-09 & 183 & $=10+39+34+28+40+32$ & 6 & Resumption from Sept. 21st (with new stepper motor) \\
\hline 2011-10 & 245 & $=40+52+44+50+31+28$ & 6 & DV897 eprom replacement \\
\hline 2011-11 & 41 & $=4+37$ & 2 & \\
\hline $2011-12$ & 29 & $=4+25$ & 2 & \\
\hline Total in 2011 & 2302 & & 60 & \\
\hline $2012-01$ & 80 & $=31+49$ & 2 & \\
\hline 2012-02 & 88 & $=33+31+24$ & 3 & \\
\hline 2012-03 & 236 & $=26+32+52+31+17+22+20+33$ & 8 & \\
\hline 2012-04 & 57 & $=17+19+21$ & 3 & \\
\hline 2012-05 & 178 & $=30+20+26+38+41+23$ & 6 & \\
\hline $2012-06$ & 79 & $=7+31+18+23$ & 4 & Maintenance of the dome \\
\hline 2012-07 & 289 & $=44+30+23+14+21+32+2+12+17+30+34$ & 11 & R.A. issues solved from July 21 st \\
\hline 2012-08 & 211 & $=29+29+31+33+31+20+28+10$ & 8 & \\
\hline 2012-09 & 147 & $=20+30+22+12+1+12+10+2+24+14$ & 10 & \\
\hline $2012-10$ & 105 & $=21+24+10+50$ & 4 & Risley prisms used from Sept. 24 th \\
\hline 2012-11 & 254 & $=20+6+54+2+52+32+30+25+33$ & 9 & \\
\hline $2012-12$ & 193 & $=50+20+2+33+38+51$ & 5 & \\
\hline Total in 2012 & 1917 & & 73 & \\
\hline
\end{tabular}



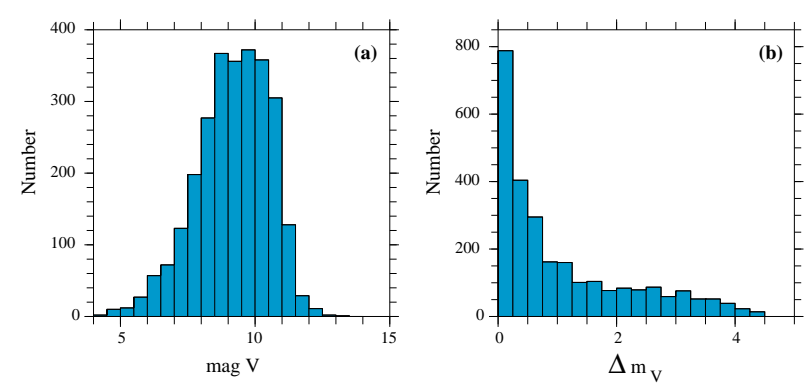

Fig. 3 Distribution of the WDS visual magnitudes of the primary components of the binary systems observed in 2011-2012 (a) and distribution of the WDS magnitude differences $\Delta m_{V}$ between the two components (b).

Fig. 3b. Those data were retrieved from the WDS catalog. The telescope aperture and detector sensitivity led to a limiting magnitude of $m_{V} \approx 14$ for the faintest companions, which corresponded to about $m_{V} \approx 12$ for the binary systems (see Fig. 3a).

Using both the photometric data contained in the WDS catalog, and the Hipparcos parallaxes, we were able to construct the color-magnitude diagram of those binaries, which is displayed in Fig. 4. Out of the 1273 objects whose parallax was measured by Hipparcos, we plotted the 974 objects for which the relative uncertainty on the Hipparcos parallax was smaller than $50 \%$. This plot shows that our observations well sampled most of the main sequence down to the faint red $\mathrm{M}$ stars. Indeed this is shown in Table 2, with the list of the 39 systems with red dwarf stars that have been observed in 2011-2012. This list was built from the spectral types found in the WDS catalog, by selecting the $\mathrm{K}$ and $\mathrm{M}$ stars of luminosity class $\mathrm{V}$ of Table 7 . It should be considered as incomplete, since precise spectral types and luminosity classes are still missing for many binaries in the WDS catalog.

\subsection{Optical setup and observing procedure}

The observations reported here were made with PISCO2 mounted on the "Grand Equatorial" of OCA Observatory. Since its free aperture diameter is $D=$ $74 \mathrm{~cm}$, the limit of diffraction is $\rho_{D}=\lambda / D=0^{\prime \prime} .16$, at $\lambda=570 \mathrm{~nm}$, corresponding to the average central wavelength of the filter we used.

The PISCO2 instrument (see sect. 2) was used here with an ANDOR iXon DV897 EMCCD camera (ANDOR, 2021). The DV897 detector is a thin back illuminated CCD chip with a very good quantum efficiency, which is cooled down $-80^{\circ}$ by an elaborate cooling system (see Paper II).

The observations presented here were obtained with a dedicated acquisition and real time processing program BuildSpeck1 that was developed by J.-L. Prieur.

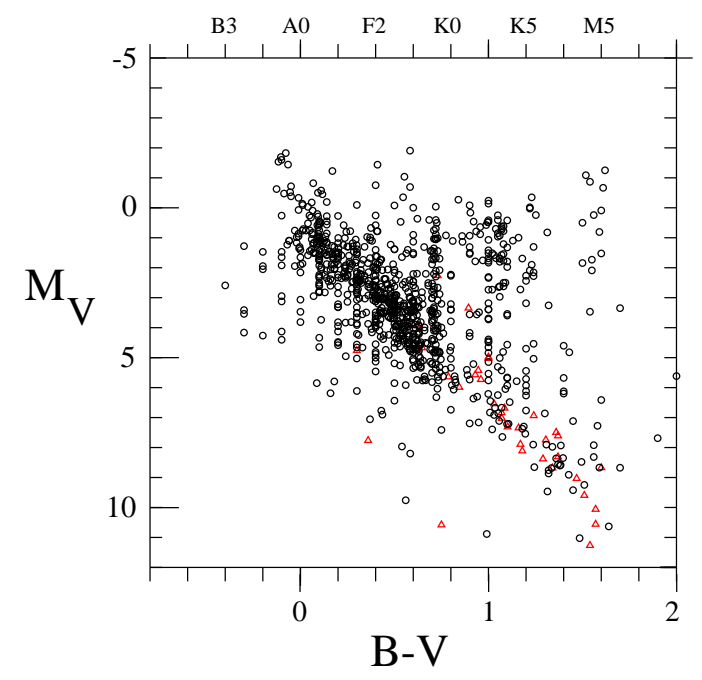

Fig. 4 Color-magnitude diagram of the binaries measured in Table 7, for which the Hipparcos parallaxes have a relative error smaller than $50 \%$ (i.e., 974 objects). In this figure, the systems containing red dwarf stars are plotted as red triangles, whereas the other objects are drawn as black circles.

This program was derived from our PISCO vcrb program that was used in Merate for the real time processing of the video analog signal produced by an ICCD camera (see Prieur et al., 2001). It also allowed a full control of all the available options of the ANDOR cameras. This program was written in $\mathrm{C}++$ language with the Borland $\mathrm{C}++$ (version 5.02) library.

Exposure times of individual frames were set in the range 20-30 msec for all objects, independently of their magnitudes. The standard format of the acquisition window on the DV897 that we used was $128 \times 128$ pixels which corresponded to a field of view of about $8^{\prime \prime} \times 8^{\prime \prime}$. For faint objects or wide pairs, a wider field on the detector was used with a binning factor of $2 \times 2$ or $4 \times 4$ which thus amounted to the same format of $128 \times 128$ pixels for the individual frames. To avoid saturation with bright objects, the EM gain was reduced and sometimes even switched off, which corresponded to observing in conventional CCD mode.

For each object, we recorded FITS cubes of 1000 frames (FITS: Flexible Image Transport System). In average $1^{\prime \prime} .2$ seeing conditions, the typical limiting $V$ magnitude was close to $13-14$ with a binning mode of $1 \times 1$.

As described in Paper I, the AF filter (anti-fringe or V-block) was mounted on the front window of the DV897 detector. This filter bandpass is $450-650 \mathrm{~nm}$ which considerably reduces the secondary spectrum of the $76-\mathrm{cm}$ refractor, with no significant loss of energy in the $V$ band. When combined with the transmission of the lenses and the quantum efficiency response of 
Table 2 List of binary systems of Table 7 that contain a red dwarf star, and data derived from the WDS with the spectral type in Col. 3, the apparent $V$ magnitude of the system in Col. 4 , its $B-V$ index in Col. 5, the difference of $V$ magnitude of the two components in Col. 6 and its absolute magnitude derived from Hipparcos parallax in Col. 7.

\begin{tabular}{|c|c|c|c|c|c|c|}
\hline WDS & Name & Spectral type & mag V & $B-V$ & $\Delta m$ & Mag V \\
\hline $00014+3937$ & HLD60 & $\mathrm{K} 0 \mathrm{~V}+\mathrm{K} 1 \mathrm{~V}$ & 8.61 & 0.79 & 0.68 & 5.16 \\
\hline $00022+2705$ & BU733 & $\mathrm{G} 5 \mathrm{Vb}+\mathrm{K} 5 \mathrm{~V}$ & 9.84 & 0.70 & 3.07 & 2.00 \\
\hline $01354+1126$ & HDS213 & $\mathrm{K} 7 \mathrm{~V}:$ & 11.82 & 1.30 & 0.08 & 6.89 \\
\hline $03035+2304$ & HDS389 & $\mathrm{F} 8 \mathrm{~V}+\mathrm{K} 0 \mathrm{~V}$ & 7.09 & 0.62 & 1.75 & 4.44 \\
\hline $03119+6131$ & HDS407 & M0V & 10.14 & 1.36 & 2.13 & 7.31 \\
\hline $04102+1722$ & HEI35 & $\mathrm{K} 2 \mathrm{~V}+\mathrm{K} 6 \mathrm{~V}$ & 9.07 & 1.07 & 1.47 & 6.45 \\
\hline $08044+1217$ & BU581 & K0V & 7.82 & 0.84 & 0.37 & 5.35 \\
\hline $08286+3502$ & WOR19 & MOV & 10.20 & 1.57 & 0.04 & 8.74 \\
\hline $09252+4606$ & HDS1353 & $\mathrm{K} 5 \mathrm{~V}+\mathrm{M} 1.5 \mathrm{~V}$ & 9.16 & 1.10 & 2.13 & 7.11 \\
\hline $10000+2433$ & CHR145 & $\mathrm{K} 0 \mathrm{~V}$ & 8.01 & 0.93 & 2.31 & 5.45 \\
\hline $11374+4728$ & KU39 & $\mathrm{K} 4 \mathrm{~V}$ & 10.19 & 1.17 & 0.48 & 7.40 \\
\hline $11544+1515$ & WOR20 & MOV & 10.50 & 1.18 & 0.21 & 7.41 \\
\hline $12223+4826$ & YSC45 & $\mathrm{K} 8 \mathrm{~V}$ & 10.39 & 1.03 & 0.10 & 6.55 \\
\hline $12422+2622$ & A1851 & $\mathrm{K} 4 \mathrm{~V}$ & 9.28 & 1.07 & 0.01 & 6.21 \\
\hline $13063+2044$ & HU739 & $\mathrm{K} 4 \mathrm{~V}$ & 9.44 & 1.29 & 2.39 & 8.09 \\
\hline $13198+4747$ & HU644 & $\mathrm{M} 2 \mathrm{~V}$ & 8.54 & 1.47 & 0.76 & 8.46 \\
\hline $13320+3108$ & WOR24 & MOV & 10.30 & 1.60 & 0.23 & 7.83 \\
\hline $13514+2620$ & SKF260 & $\mathrm{K} 6 \mathrm{~V}$ & 11.00 & 1.37 & 2.50 & 7.61 \\
\hline $13514+2620$ & YSC50 & $\mathrm{K} 6 \mathrm{~V}$ & 11.00 & 1.37 & 0.40 & 7.61 \\
\hline $14127+2349$ & YSC53 & $\mathrm{K} 0 \mathrm{~V}$ & 8.88 & 0.96 & 4.10 & 6.31 \\
\hline $15038+4739$ & STF1909 & $\mathrm{F} 7 \mathrm{~V}+\mathrm{K} 4 \mathrm{~V}$ & 4.83 & 0.65 & 0.90 & 4.30 \\
\hline $15360+3948$ & STT298 & $\mathrm{K} 2 \mathrm{~V}$ & 7.65 & 0.95 & 1.28 & 5.91 \\
\hline 17067-0749 & RST3965 & $\mathrm{K} 5 \mathrm{~V}$ & 10.27 & 1.08 & 0.63 & 6.11 \\
\hline $17247+3802$ & HSL1 & $\mathrm{G} 5 \mathrm{~V}+\mathrm{K} 6 \mathrm{~V}$ & 8.60 & 0.30 & 2.50 & 5.08 \\
\hline $17372+2754$ & KUI83 & MoV:p & 9.20 & 1.16 & 0.59 & 6.73 \\
\hline $17465+2743$ & $\mathrm{AC} 7$ & M3.5V & 3.41 & 0.75 & 0.50 & 3.79 \\
\hline $18500+1519$ & YSC12 & $\mathrm{F} 8+\mathrm{M} 2 \mathrm{~V}$ & 7.53 & 0.62 & 3.70 & 3.85 \\
\hline $19074+3230$ & KUI90 & M3V & 5.21 & 0.36 & 0.59 & 2.17 \\
\hline $19121+0254$ & AST1 & M3.5V & 11.09 & 1.54 & 1.82 & 11.05 \\
\hline $19347+2854$ & COU1031 & $\mathrm{K} 0 \mathrm{~V}$ & 8.60 & 1.00 & 3.25 & 4.68 \\
\hline $19347+2854$ & GII33 & $\mathrm{K} 0 \mathrm{~V}$ & 8.60 & 1.00 & 1.80 & 4.68 \\
\hline $20302+2651$ & WOR9 & M1V & 9.69 & 1.34 & 0.13 & 7.87 \\
\hline $20396+0458$ & KUI99 & $\mathrm{K} 5 \mathrm{~V}$ & 7.88 & 1.24 & 1.35 & 6.54 \\
\hline $21000+4004$ & KUI103 & $\mathrm{M} 2 \mathrm{~V}+\mathrm{M} 0.5 \mathrm{~V}$ & 10.14 & 1.51 & 1.91 & 9.24 \\
\hline $22234+3228$ & WOR11 & M3.0V & 10.73 & 1.57 & 0.42 & 9.70 \\
\hline $22281+1215$ & BU701 & $\mathrm{K} 0 \mathrm{~V}$ & 7.10 & 0.89 & 2.28 & 3.10 \\
\hline $22536+3756$ & RUC15 & $\mathrm{G} 5 \mathrm{~V}+\mathrm{K} 9 \mathrm{~V}$ & 8.95 & 0.73 & 2.59 & 4.40 \\
\hline $23167+3441$ & HDS3315 & $\mathrm{K} 8 \mathrm{~V}$ & 10.50 & 0.81 & 0.50 & 5.61 \\
\hline $23405+2959$ & HDS3363 & $\mathrm{K} 4 \mathrm{~V}$ & 10.40 & 1.15 & 2.82 & 6.55 \\
\hline
\end{tabular}

the detector, the resulting transmission curve is close to a standard $V$ filter with a maximum transmission at around $570 \mathrm{~nm}$ (see Paper I).

\section{Scale and position angle calibration}

The astrometric calibration of the magnification scale of PISCO2 was done with a calibration grating mask (see Paper II). The scale value was found to be $0^{\prime \prime} .0738 /$ pixel when the frames were not binned $(x b i n=1, y b i n=1)$. In that case, the diffraction limit in $V$ of $0^{\prime \prime} .16$ (see Sect. 3.2) corresponds to 2.17 pixels, which thus is in agreement with Shannon's sam- pling criteria (i.e. the diffraction diameter is sampled by more than two pixels).

The calibration of the origin of the position angles was done by recording star trails caused by the diurnal motion. The orientation of our images on the detector was approximately conventional, with North to the bottom, and East to the right. After measuring a series of star trail images, we found: $\theta_{\text {sky }}=90.99+\theta_{\text {frame }}$, where $\theta_{\text {sky }}$ and $\theta_{\text {frame }}$ are the angular positions on the sky and on the EMCCD frame in degrees, respectively. 

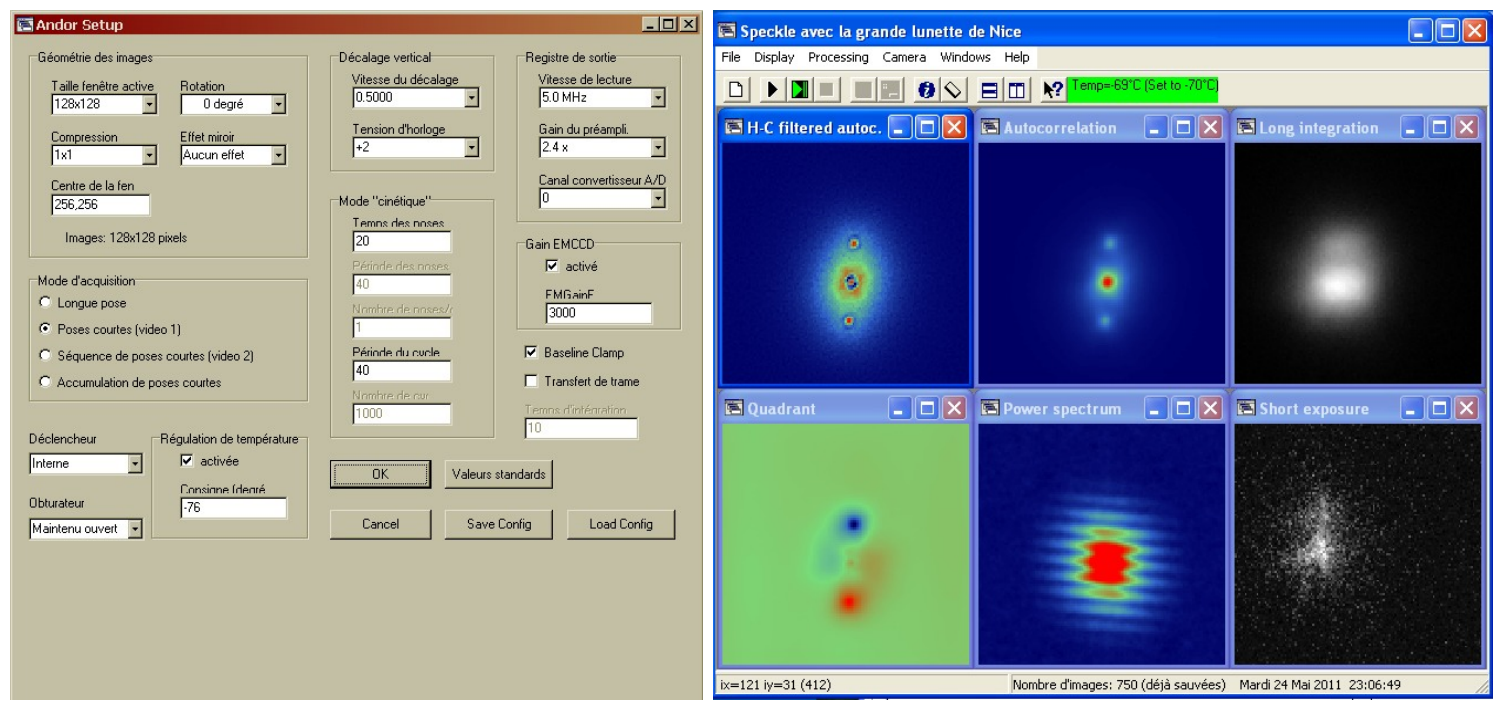

Fig. 5 Program Buildspeck1 used for data acquisition with ANDOR cameras and real-time processing. Left: camera setup; right: example of image processing of the binary star BU $1273\left(\rho=1 .^{\prime \prime} 3, m_{V}=9.6 \Delta m_{V}=1.3\right)$.

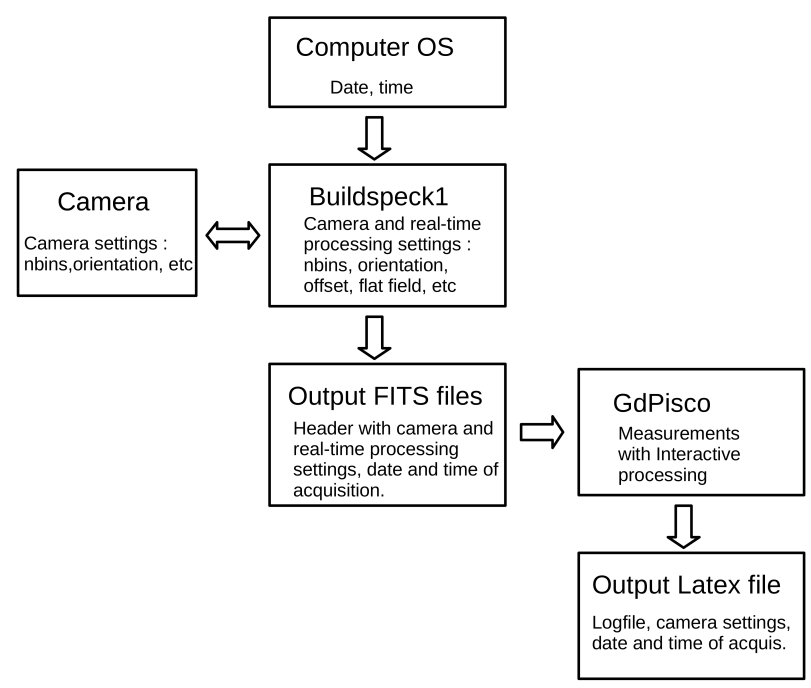

Fig. 6 Overview of the data acquisition and processing pipeline used for deriving the binary measurements. We have written two specific programs: Buildspeck1 for the acquisition and real-time processing and GdPisco for interactive processing and final measurements.

\section{Data reduction procedure}

The pioneering work of Labeyrie (1970) showed that high resolution information could be extracted from short-exposures by computing the average power spectrum of those exposures. This technique is known as "speckle interferometry" and has proved to be very efficient to observe binary stars even in poor seeing conditions. The observations presented here have been processed in this way. More precisely, we have used the method described in Worden et al.(1977), which works with a "flattened" autocorrelation, and is routinely used for PISCO (see Scardia et al., 2019, for instance). As shown in Fig. 6, the whole processing was done in two steps: real-time processing with the BuildSpeck1 program and post-processing with programs like Gdpisco.

An example of the display panels produced by BuildSpeck1 are given in Fig. 5. This program both controls the camera and processes the data in real time by pre-processing the individual frames (with offset and flat-field corrections), computing Fast Fourier Transforms and generating all the average frames that are needed by our reduction procedure (for speckle analysis, or the Lucky-Imaging and Direct Vector Autocorrelation methods).

The Gdpisco program leads to final measurements using both the data and some information contained in the headers of the mean autocorrelation FITS files produced by BuildSpeck1. Indeed, those headers contain the epoch of observation, and some settings that have been used during the observations, like the binning factor of the images (for instance, a value of bin $=2$ indicates that one final pixel is $2 \times 2$ initial pixels).

As in Paper II, the $180^{\circ}$-ambiguity of speckle measurements related to the position angles of binaries was mostly solved by the direct vector autocorrelation (DVA) method, as proposed by Bagnuolo et al (1992), which computes the "oriented" autocorrelation function and takes into account the brightness level of the vector ends. The DVA both allows the quadrant determination and the measurement of $\Delta m$ when the contrast is not too large (e.g. $\Delta m<2$ ).

In the case of good seeing conditions, we obtained high resolution images with the Lucky Imaging method implemented in the REDUC software (Losse, 2021). This method was described in Paper I, and consists of shift-and-add processing a subset of selected good images. We experimentally found that a selection of about 
3 to $10 \%$ of the best images of our 1000-frame cubes often led to very nice shift-and-add images, with clear Airy rings and a final resolution close to the diffraction limit of the $76-\mathrm{cm}$ refractor. We could measure couples with very large differences of brightness, up to 3-4 magnitudes (see Sect. 6). Compared to the speckle interferometry, the main advantage of "Lucky imaging" is to provide a full image which is convenient for the determination of the quadrants (where the companion stands) and of the difference of magnitudes between the two components (see the example of Sect. 8).

\section{Relative astrometric and photometric measurements}

The relative astrometric and photometric measurements that we obtained with PISCO2 in 2011-2012 are displayed in Table 7 . They concern 3136 visual binaries or multiple objects, with 3568 position measurements and 308 cases of unresolved observations.

In this table, for each binary we successively give its WDS name in Col. 1, the official double star designation in Col. 2 (sequence is "discoverer-number", with 'ND' added in Col. 10 when it is a new double), the epoch of observation in Besselian years (Col. 3), the binning factor used by the detector when acquiring the image (Col. 4), the angular separation $\rho$ (Col. 5) between the two components with its error (Col. 6) in arcseconds, and the position angle $\theta$ (Col. 7) with its error (Col. 8) in degrees, measured from the North and positive to the East, the measured difference of magnitudes $\Delta m$ between the two components (Col. 9), and some notes in Col. 10 ('ND' for 'New Double' or 'NR' for 'Not Resolved').

The $(O-C)$ (Observed minus Computed) residuals of the measurements for the systems with a known orbit are displayed in Cols. 12 and 13 for the residuals in angular separation and position angle, respectively. The orbital elements used for computing the ephemerides were retrieved from the "Sixth Catalogue of Orbits of Visual Binary Stars" (Hartkopf et al., 2001, and Matson et al., 2021, hereafter OC6). The corresponding authors are given in Col. 11, using the style of the OC6 references. For some objects, we have taken into account the orbits computed by Tokovinin (2021), that have not been inserted into the OC6 yet.

The difference of magnitudes $\Delta m$ with the wideband filter that we used for the observations (see Paper I), was obtained either from the Lucky Imaging or the DVA method, is reported in Col. 9, and concerns 326 objects (see Fig. 7b). By comparing measurements obtained for the same objects, we estimated the average (internal) errors at about 0.2 mag.

The distribution of the 3568 angular separations measured in this paper is displayed in Fig. 7a and
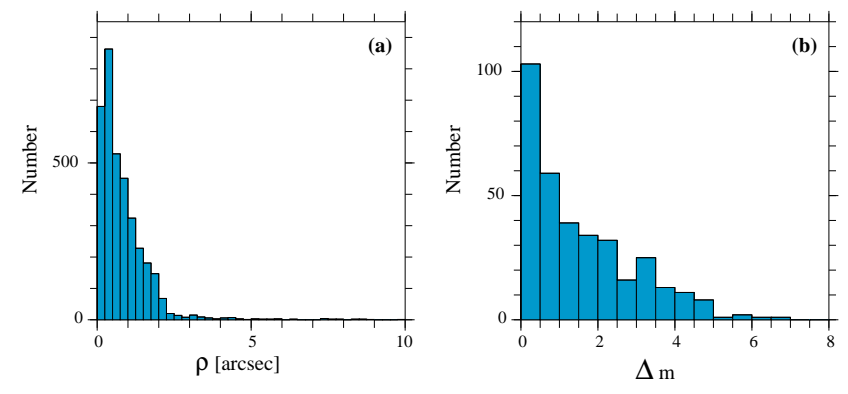

Fig. 7 Distribution of the angular separations of the measurements of Table 7 (a), and of the differences of magnitude $\Delta m$ measured with Lucky Imaging and direct vector methods (b).

shows a maximum for $\rho \approx 0^{\prime \prime} .4$. The largest separation of $\rho=10^{\prime \prime} .3$ was obtained for CON37A,CD. The smallest separation was measured for HDS2639 with $\rho=0^{\prime \prime} .08$. This is smaller than the diffraction limit $\rho_{D}=\lambda / D=0^{\prime \prime} .16$ (see Sect. 3.2), with our $V$ filter (i.e. $\lambda=570 \mathrm{~nm}$ ) and our refractor whose free aperture is $D=0.74 \mathrm{~m}$. The residuals of our measurements with published orbits will be discussed in Sect. 7 .

A superscript ${ }^{*}$ was added to $\theta$ measurements in Col. 7 when we could determine the quadrants of the measurements, i.e., when our observations allowed us to solve the $180^{\circ}$ ambiguity of the autocorrelation frames. When this was not possible, we used the quadrant of the last observations from the WDS catalog. Eventually when such observations were missing, we assumed that the quadrant was equal to 1 or 2 (i.e. the companion was lying in the $\left[0^{\circ}, 90^{\circ}\right]$ or $\left[90^{\circ}, 180^{\circ}\right]$ sectors).

A superscript ${ }^{Q}$ was added to $\theta$ residuals in Col. 13 when the quadrants of our measurements were not in agreement with those used for the orbits.

The average errors determined by our Gdpisco program are $0^{\prime \prime} .016 \pm 0^{\prime \prime} .019$ and $1^{\circ} .7 \pm 2^{\circ} .6$ for $\rho$ and $\theta$ respectively. Those errors were computed for each measurement by estimating the uncertainties on the background estimation and are consistent with the variations seen in repeat measurements of the same systems. Indeed the background removal on the autocorrelation spots is the critical step of our measurement procedure. Since the frame scale was $0^{\prime \prime} .0738 /$ pixel, those values indicate that the location of the secondary peaks of the autocorrelations was determined with a mean accuracy of about $1 / 5$ th of a pixel.

During our observations we have found some new stellar components around double stars that were previously known. This concerns 47 measurements of 28 new double stars that we discovered in 2011-2012, and it is indicated by "ND" in Col. 10 of Table 7. The subset of those measurements is reported in Table 4. This table has the same column description as Table 7 , which was described in the beginning of this section. Some 
Table 3 Table of speckle measurements obtained with PISCO2 in 2011-2012 and O-C residuals with published orbits (begin.)

\begin{tabular}{|c|c|c|c|c|c|c|c|c|c|c|c|c|}
\hline WDS & Name & Epoch & Bin. & $\begin{array}{c}\rho \\
(")\end{array}$ & $\begin{array}{l}\sigma_{\rho} \\
(")\end{array}$ & $\begin{array}{c}\theta \\
\left(^{\circ}\right)\end{array}$ & $\begin{array}{l}\sigma_{\theta} \\
\left(^{\circ}\right)\end{array}$ & $\Delta m$ & Notes & Orbit & $\begin{array}{r}\Delta \rho(\mathrm{O}-\mathrm{C}) \\
(")\end{array}$ & $\begin{array}{r}\Delta \theta(\mathrm{O}-\mathrm{C}) \\
\left({ }^{\circ}\right)\end{array}$ \\
\hline $00003+6557$ & MLR280 & 2012.988 & 1 & 1.512 & 0.021 & $9.6^{*}$ & 0.3 & & & & & \\
\hline $00004+2749$ & TDS1238 & 2012.840 & 1 & 0.818 & 0.007 & $86.4^{*}$ & 0.3 & & & & & \\
\hline $00008+1659$ & BAG18 & 2012.840 & 1 & 0.674 & 0.007 & 177.5 & 0.4 & & & Tok2019c & 0.00 & 4.7 \\
\hline $00014+3937$ & HLD60 & 2011.582 & 1 & 1.324 & 0.007 & $168.9^{*}$ & 0.3 & & & Izm2019 & 0.02 & 0.5 \\
\hline $00022+2705$ & BU733AB & 2011.582 & 1 & 0.782 & 0.007 & 286.8 & 0.3 & & & Sod1999 & 0.02 & -0.5 \\
\hline $00022+2705$ & BU733AB & 2011.830 & 1 & 0.718 & 0.007 & $287.8^{*}$ & 0.3 & & & Sod1999 & -0.02 & -2.0 \\
\hline $00022+2705$ & BU733AB & 2011.830 & 1 & 0.720 & 0.007 & 288.0 & 0.3 & & & Sod1999 & -0.02 & -1.8 \\
\hline $00022+2705$ & BU733AB & 2012.854 & 1 & 0.667 & 0.007 & 300.3 & 0.3 & & & Sod1999 & 0.03 & -1.8 \\
\hline $00024+1047$ & A1249 & 2011.751 & 1 & 0.235 & 0.011 & $257.3^{*}$ & 1.8 & & & Zir2003 & 0.06 & 4.7 \\
\hline $00026-0829$ & A428 & 2011.751 & 1 & 0.107 & 0.030 & 337.8 & 3.5 & & & Tok2015c & -0.03 & 5.6 \\
\hline $00026+1841$ & $\mathrm{HDS} 2 \mathrm{Aa}, \mathrm{Ab}$ & 2011.830 & 1 & 0.156 & 0.040 & 150.5 & 8.0 & & & & & \\
\hline $00026+1841$ & $\mathrm{HDS} 2 \mathrm{Aa}, \mathrm{Ab}$ & 2012.840 & 1 & 0.156 & 0.040 & 144.4 & 8.0 & & & & & \\
\hline $00026+1841$ & $\mathrm{HDS} 2 \mathrm{Aa}, \mathrm{Ab}$ & 2012.854 & 1 & 0.126 & 0.040 & 149.7 & 8.0 & & & & & \\
\hline $00026+1841$ & HDS2AB & 2011.830 & 1 & 2.015 & 0.014 & 337.3 & 0.3 & & & & & \\
\hline $00026+1841$ & HDS2AB & 2012.854 & 1 & 2.026 & 0.010 & 337.4 & 0.3 & & & & & \\
\hline $00026+1841$ & HDS2AB & 2012.854 & 1 & 2.072 & 0.010 & $336.6^{*}$ & 0.3 & 4.69 & & & & \\
\hline $00039+2759$ & $\mathrm{~A} 429 \mathrm{AB}$ & 2011.582 & 1 & 0.542 & 0.007 & $331.4^{*}$ & 0.3 & & & & & \\
\hline $00039+2759$ & HJ1929AB,C & 2011.582 & 1 & 4.684 & 0.023 & $249.9^{*}$ & 0.3 & & & & & \\
\hline $00047+3547$ & HU800 & 2011.759 & 1 & 0.204 & 0.013 & $246.6^{*}$ & 1.0 & & & & & \\
\hline $00054+3348$ & COU646 & 2012.840 & 1 & 0.468 & 0.007 & $150.8^{*}$ & 0.3 & & & & & \\
\hline $00055+3406$ & HU1201 & 2011.582 & 1 & 0.185 & 0.040 & $305.6^{*}$ & 8.0 & & & Zir2003 & -0.00 & -1.5 \\
\hline $00055+3406$ & HU1201 & 2012.854 & 1 & 0.158 & 0.040 & 312.7 & 8.0 & & & Zir2003 & -0.02 & 5.7 \\
\hline $00058+2855$ & HEI831 & 2011.584 & 1 & 1.897 & 0.011 & $136.4^{*}$ & 0.4 & & & & & \\
\hline $00068+0427$ & BU1155 & 2012.980 & 1 & 0.662 & 0.007 & $75.9^{*}$ & 0.4 & & & & & \\
\hline $00073+0742$ & HDS13 & 2012.980 & 1 & 0.376 & 0.007 & $141.6^{*}$ & 0.3 & & & & & \\
\hline $00073+2058$ & HDS12 & 2012.840 & 1 & 1.872 & 0.009 & $7.4^{*}$ & 0.3 & & & & & \\
\hline $00089+1332$ & TDS1313 & 2012.980 & 1 & 0.810 & 0.015 & $121.9^{*}$ & 0.8 & & & & & \\
\hline $00092+0937$ & TDS1320 & 2012.980 & 1 & 0.596 & 0.007 & $217.6^{*}$ & 0.5 & & & & & \\
\hline
\end{tabular}

Notes: In column 7, the superscript ${ }^{*}$ indicates that the position angle $\theta$ could be determined without the $180^{\circ}$ ambiguity. In column 13, the superscript ${ }^{Q}$ indicates that our $\theta$ measurement quadrant was not compatible with the quadrant used for computing the orbit.

of them have been monitored since their discovery and were added to the list of René Gili's new double stars discoveries (Gili, 2016). In Table 4, they correspond to the objects whose names start with GII. More observations are required to confirm the other new couples of this table.

\section{Residuals from published orbits}

\subsection{Comparison with published ephemerides}

The residuals from published orbits that have been computed with our measurements of Table 7 are plotted in Fig. 9a. Among those 625 residuals, this plot underlines the presence of eight objects with very large residuals, that will be discussed in Sect. 7.2: STF148, HO366AB, A2157, STT260, A700, COU1006, HU767, and HU374. The other residuals are well centered around the origin, with a rather large scatter.
The mean values computed with the residuals of Table 7 are $\left\langle\Delta \rho_{O-C}>=0^{\prime \prime} .007 \pm 0^{\prime \prime} .047\right.$ and $<$ $\Delta \theta_{O-C}>=-0^{\circ} .01 \pm 3^{\circ} .9$ (after rejecting the outliers). In both cases, the offsets are very close to zero, with absolute values much smaller than the standard deviations which provides a validation of our calibration (see Sect. 3.2). The rather large standard deviation that can be explained by the poor quality of many orbits due to their old age and the lack of observations. Indeed we have checked that our measurements are in good agreement with the observations reported in the "Fourth Catalogue of Interferometric Measurements of Binary Stars" (Hartkopf et al., 2001, and Hartkopf et al., 2021, hereafter IC4), when they are present in this catalog.

The measurements with the largest residuals of Table 7 , such that $\Delta \rho>0.1^{\prime \prime}$ or $\Delta \theta_{O-C}>10^{\circ}$, are reported in Table 5. Some examples of autocorrelations of 
Table 4 Table of speckle measurements of new double stars: new measurements of the 28 'ND' objects that were discovered while observing known binaries.

\begin{tabular}{|c|c|c|c|c|c|c|c|c|c|}
\hline WDS & Name & Epoch & Bin. & $\begin{array}{c}\rho \\
(")\end{array}$ & $\begin{array}{l}\sigma_{\rho} \\
(")\end{array}$ & $\begin{array}{c}\theta \\
\left({ }^{\circ}\right) \\
\end{array}$ & $\begin{array}{c}\sigma_{\theta} \\
\left({ }^{\circ}\right)\end{array}$ & $\Delta m$ & Notes \\
\hline $04334+1949$ & A2351AC & 2011.113 & 1 & 3.076 & 0.015 & 173.1 & 0.3 & 3.18 & \\
\hline $06283+2441$ & COU914AC & 2012.142 & 2 & 8.911 & 0.045 & $28.8^{*}$ & 0.3 & & \\
\hline $08567+0130$ & GII39AC & 2011.269 & 1 & 1.806 & 0.012 & 54.9 & 0.3 & & In HEI745 system \\
\hline $08567+0130$ & HEI745AC & 2012.000 & 1 & 1.030 & 0.015 & 174.4 & 0.5 & & ND \\
\hline $09062+3314$ & GII37Ba,Bb & 2011.179 & 1 & 0.340 & 0.007 & 88.6 & 0.3 & 0.06 & In COU1560 system \\
\hline $09062+3314$ & GII37Ba,Bb & 2011.179 & 1 & 0.342 & 0.007 & 88.7 & 0.3 & 0.06 & In COU1560 system \\
\hline $09062+3314$ & GII37Ba,Bb & 2011.184 & 1 & 0.338 & 0.007 & 89.6 & 0.3 & 0.10 & In COU1560 system \\
\hline $09062+3314$ & GII37Ba,Bb & 2011.184 & 1 & 0.331 & 0.007 & 90.1 & 0.3 & 0.10 & In COU1560 system \\
\hline $09062+3314$ & GII37Ba,Bb & 2012.252 & 1 & 0.323 & 0.007 & $94.4^{*}$ & 0.3 & 0.03 & In COU1560 system \\
\hline $09094+2203$ & WRH16AC & 2011.135 & 1 & 2.167 & 0.013 & 8.2 & 0.4 & & \\
\hline $11551+4629$ & A1777AC & 2011.253 & 1 & 3.905 & 0.020 & $41.8^{*}$ & 0.3 & 2.46 & \\
\hline $11551+4629$ & A1777AC & 2011.253 & 1 & 3.900 & 0.020 & $41.7^{*}$ & 0.3 & & \\
\hline $13203+1746$ & A2166AC & 2011.384 & 1 & 2.170 & 0.011 & $225.8^{*}$ & 0.3 & & \\
\hline $13276+2116$ & GII60AC & 2012.361 & 1 & 2.881 & 0.014 & 127.8 & 0.3 & & ND \\
\hline $13276+2116$ & GII60AC & 2012.362 & 1 & 2.897 & 0.014 & 127.9 & 0.3 & 3.83 & ND \\
\hline $13276+2116$ & GII60AC & 2012.397 & 1 & 2.896 & 0.014 & 126.9 & 0.3 & 4.22 & ND \\
\hline $14111+3628$ & GII96Aa,Ab & 2011.387 & 1 & 0.356 & 0.007 & 192.4 & 0.6 & & ND \\
\hline $15371+2646$ & HDS2199AC & 2011.469 & 1 & 2.093 & 0.012 & 62.9 & 0.3 & & \\
\hline $16211+0250$ & HEI794AC & 2011.521 & 2 & 3.749 & 0.037 & $33.8^{*}$ & 0.3 & & \\
\hline $16213+7434$ & HDS2310AC & 2011.515 & 1 & 2.698 & 0.013 & 26.7 & 0.3 & & \\
\hline $16213+7434$ & HDS2310AC & 2012.523 & 1 & 2.706 & 0.014 & $25.4^{*}$ & 0.3 & & \\
\hline $16213+7434$ & HDS2310AC & 2012.523 & 1 & 2.733 & 0.014 & 26.3 & 0.5 & & \\
\hline $17006+2002$ & COU199AC & 2011.556 & 1 & 1.300 & 0.007 & $331.4^{*}$ & 0.4 & & \\
\hline $17532+3243$ & $\mathrm{GII} 43 \mathrm{Aa}, \mathrm{Ab}$ & 2011.556 & 1 & 0.261 & 0.010 & 29.7 & 2.0 & & In COU998 system \\
\hline $17532+3243$ & GII43Aa,Ab & 2011.576 & 1 & 0.178 & 0.040 & 25.5 & 8.0 & 0.30 & In COU998 system \\
\hline $17532+3243$ & GII43Aa, Ab & 2012.479 & 1 & 0.187 & 0.040 & 22.4 & 8.0 & 0.47 & In COU998 system \\
\hline $17532+3243$ & GII43Aa,Ab & 2012.518 & 1 & 0.244 & 0.007 & 18.3 & 1.1 & & In COU998 system \\
\hline $18360+3452$ & GII48 & 2012.630 & 1 & 0.345 & 0.007 & $315.3^{*}$ & 0.3 & & Very close to TDT953 \\
\hline $18360+3452$ & GII48 & 2012.632 & 1 & 0.359 & 0.010 & $316.1^{*}$ & 0.6 & & Very close to TDT953 \\
\hline $19240+2437$ & TDS988AC & 2012.605 & 1 & 3.279 & 0.016 & 55.6 & 0.3 & & \\
\hline $19240+2437$ & TDS988AC & 2012.627 & 1 & 3.305 & 0.017 & $56.6^{*}$ & 0.3 & & \\
\hline $19264+2241$ & GII50 & 2012.641 & 1 & 0.396 & 0.010 & $184.8^{*}$ & 0.3 & & Close to CON37 \\
\hline $19264+2241$ & GII50 & 2012.682 & 1 & 0.385 & 0.007 & 187.8 & 1.0 & & Close to CON37 \\
\hline $20135+3526$ & COU2214AC & 2011.573 & 1 & 3.420 & 0.018 & $263.6^{*}$ & 0.3 & & \\
\hline $20135+3526$ & COU2214AC & 2011.576 & 1 & 3.404 & 0.017 & $263.3^{*}$ & ${ }^{k} 0.3$ & & \\
\hline $20201+3700$ & TDT2183AC & 2012.755 & 2 & 2.922 & 0.029 & 258.8 & 0.3 & 1.79 & \\
\hline $20205+1634$ & HU360AC & 2011.748 & 2 & 7.327 & 0.037 & $347.8^{*}$ & 0.3 & & \\
\hline $20205+1634$ & HU360AC & 2011.751 & 2 & 7.324 & 0.037 & $348.2^{*}$ & 0.3 & & \\
\hline $20236+3854$ & GII51 & 2012.725 & 1 & 3.246 & 0.016 & $355.8^{*}$ & ${ }^{*} 0.3$ & & Close to TDT2232 \\
\hline $20236+3854$ & GII51 & 2012.728 & 1 & 3.192 & 0.016 & $356.6^{*}$ & ${ }^{k} 0.3$ & & Close to TDT2232 \\
\hline $20359+3617$ & GII52Aa,Ab & 2012.726 & 1 & 0.354 & 0.007 & $216.2^{*}$ & * 0.8 & & In TDT2405 system \\
\hline $21138+3711$ & COU1816AC & 2012.726 & 2 & 10.038 & 0.050 & $336.1^{*}$ & ${ }^{*} 0.3$ & & \\
\hline $22360+4515$ & GII55 & 2012.868 & 2 & 2.523 & 0.025 & $311.6^{*}$ & ${ }^{*} 0.6$ & & Close to COU2242 \\
\hline $23033+1129$ & GII71Aa,Ab & 2012.889 & 1 & 0.139 & 0.040 & 30.7 & 8.0 & & \\
\hline $23110+3817$ & COU1199BC & 2011.584 & 1 & 0.853 & 0.007 & 65.4 & 0.3 & 1.63 & \\
\hline $23342+0759$ & TDT4102AC & 2012.889 & 1 & 1.158 & 0.007 & 46.8 & 0.3 & 4.41 & \\
\hline $23342+0759$ & TDT4102AC & 2012.889 & 1 & 1.158 & 0.007 & 46.2 & 0.3 & & \\
\hline
\end{tabular}

Note: In column 7 , the superscript ${ }^{*}$ indicates that the position angle $\theta$ could be determined without the $180^{\circ}$ ambiguity.

those binaries are shown in Fig. 8. The content of this table has the same notation as Table 7. In Col. 11, we reported some additional measurements obtained with PISCO2 in 2012-2016 and PISCO in 2020 (unpublished data yet), or from the IC4. Those measurements are noted as "P" followed by the epoch for the observations obtained with PISCO or PISCO2, or "IC", followed by the epoch for IC4 measurements.

In Table 5, we see that our measurements are in fair agreement with the additional measurements of Col. 11, even when the residuals are large, as we will see in the next section. The large residuals are thus an indication that the orbits are still not definitive. Indeed most of those objects have been poorly monitored until now, and the orbits still need additional measurements to be more precise.

If we consider the best orbits only, for instance the orbits of Grade 1 in OC6, the plot of the residuals of Table 7 becomes much more concentrated (see Fig. 9b). This shows that the dispersion of Fig. 9a is caused by the poor quality of most of the orbits. 
Table 5 Measurements made in 2011-2012 with large residuals: values with $\left|\rho_{O-C}\right|>0^{\prime \prime} .1$ or $\left|\theta_{O-C}\right|>10^{\circ}$.

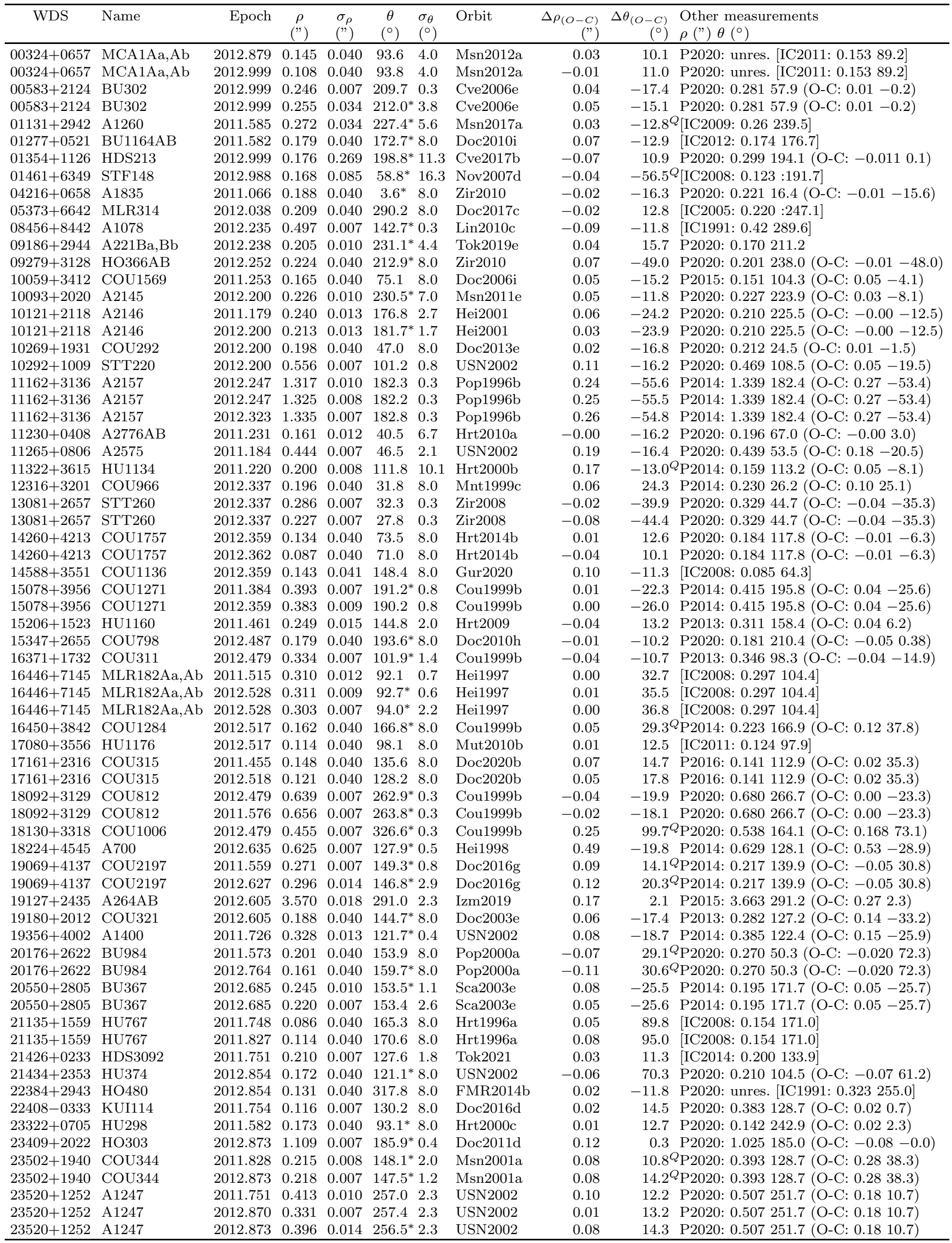




\subsection{Discussion on the few cases of very large residuals}

We discuss here the cases of measurements with very large residuals, that appear as outliers in Fig. 9a, i.e., STF148, HO366AB, A2157, STT260, A700, COU1006, HU767, and HU374.

The case of A700 is particular as the residuals over the last years have been increasing with time. As reported in the notes in the IC4: "Increasing residuals since the orbital solution by Heintz (1998) led to the determination by Parent et al. (2017) that the relative motion of the pair was better represented by a rectilinear fit. Thus the pair appears to be an optical double rather than a physical binary. Rectilinear solution was computed by Parent et al. (2017)."

For the other objects of that list with very large residuals, the observations are scarce in the IC4, and only small arcs of orbits have been monitored until now. As shown in Col. 11 of Table 5, our measurements are consistent with the others that are reported in the IC4 and with the other measurements made by PISCO and PISCO2 for similar epochs. Clearly more measurements are needed for obtaining reliable orbits of those objects.

\subsection{Measurements under the diffraction limit}

In Table 7, there are 196 measurements with an angular separation smaller than $0.16^{\prime \prime}$, which is the diffraction limit of our instrumentation (see Sect. 3.2). Some examples of autocorrelations of those binaries that have been processed with our high contrast numerical filters are shown in Fig. 11. As expected the autocorrelation peaks are not clearly divided, although the central peak is elongated. Our measurement was made by assuming that the lateral peaks were located on the very edges of that elongated peak.

When an orbit was known, we reported those measurements and the corresponding residuals in Table 6 , with the same notation as in Table 7 . In the last column, we also reported some additional measurements obtained with PISCO in 2020, and with PISCO2 in 2012-2016, or from the IC4, with the same notation as in Table 5 .

The mean value of the angular separation from the 60 measurements of this table is $\langle\rho\rangle=0^{\prime \prime} .133 \pm$ $0^{\prime \prime} .020$. With a scale of $0.0738^{\prime \prime} /$ pixel (see Sect. 4), this value corresponds to 1.8 pixels on the autocorrelation frames. The location of the autocorrelation peaks could be estimated with an average error of $0^{\prime \prime} .016$ (see Sect. 6). Hence the average error on the position angle corresponds to the angle of $0.016^{\prime \prime}$ seen at the distance of $0.133^{\prime \prime}$, which is therefore: $\arctan (0.016 / 0.133) \approx 7^{\circ}$.

The residuals from published orbits of the measurements of Table 6 are plotted in Fig. 10. We notice the presence of an outlier, HU767, that has been discussed in the previous section.
The mean values computed with the 60 residuals of Table 6 are $\left\langle\Delta \rho_{O-C}\right\rangle=-0^{\prime \prime} .002 \pm 0^{\prime \prime} .044$ and $<\Delta \theta_{O-C}>=2^{\circ} .2 \pm 7^{\circ} .6$ (after rejecting the outliers). Our measurements under the diffraction limit are therefore in good agreement with the ephemerids of published orbits, even though the uncertainties are larger in this case (as shown above, the expected average errors are $0^{\prime \prime} .02$ for $\rho$, and $7^{\circ}$ for $\theta$ ). Those measurements have been made possible by good observations obtained with good seeing conditions, and by the use of efficient high contrast filters. Those filters were proposed by R. Gili and implemented in the PISCO and PISCO2 programs by J.-L. Prieur. They are based on strioscopic Fourier filtering, which blocks the low frequency continuous component of the images.

\section{Detailed study of CON37}

We observed with PISCO2 the faint multiple system CON37 that was discovered in 2004 with the UKIRT $3.8 \mathrm{~m}$-telescope (Connelley et al., 2008). The global magnitude of the AB pair is $m_{V}=15.5$ (SIMBAD, 2021), which is at the limit of our instrumentation.

We confirmed the detection of the $\mathrm{AB}$ and $\mathrm{CD}$ components in the visible, when rather good seeing conditions allowed us to obtain some "Lucky" images of CON37 (see Fig. 12). Those images were processed with the IRIS software (Buil, 2021), in particular with wavelets filters.

CON37-AB was well resolved with PISCO2 on our images obtained on Aug 9th 2012 for example. We obtained the following measurement: $\left(\theta=325.3^{\circ}, \rho=1.556\right.$, $\Delta m \approx 2.1$, with the B component in the 4th quadrant, whereas those components had the same brightness in $K$ (Connelley et al. 2008). The CD pair which has an estimated magnitude $m_{V} \approx 18.5$, was detected on our images. It could not be measured accurately on that night, but seemed to have slightly rotated, when we compared with the observations of Connelley et al. (2008).

We also discovered a new couple that is located in the South-Western neighborhood of CON37 (see Fig. 13), which we called GII $50(192640.720+224127.72$, see Gili, 2016).

\section{Conclusion}

In 2011-2012, we obtained 3568 new position measurements of 3136 visual binaries or multiple systems with the $76-\mathrm{cm}$ refractor in Nice, with an average accuracy of $0^{\prime \prime} .02$ for the angular separations and $1^{\circ} .7$ for the position angles. We have been able to routinely monitor faint systems $m_{V}=9-11$ and the color-magnitude diagram of our observations shows that we have thus been able to measure red dwarf stars that had been poorly 
Table 6 Measurements and residuals of the closest binaries observed in 2011-2012, with $\rho<0$ " .16 (i.e. smaller than the diffraction limit), for the objects having a known orbit.

\begin{tabular}{|c|c|c|c|c|c|c|c|c|}
\hline WDS & Name & Epoch & $\begin{array}{c}\rho \\
(")\end{array}$ & $\begin{array}{c}\theta \\
\left({ }^{\circ}\right) \\
\end{array}$ & Orbit & $\begin{array}{r}\Delta \rho(\mathrm{O}-\mathrm{C}) \\
(")\end{array}$ & $\begin{array}{r}\Delta \theta(\mathrm{O}-\mathrm{C}) \\
\left({ }^{\circ}\right)\end{array}$ & $\begin{array}{l}\text { Other measurements } \\
\rho(") \theta\left(^{\circ}\right)\end{array}$ \\
\hline $00026-0829$ & $\mathrm{~A} 428$ & 2011.751 & 0.107 & 337.8 & Tok2015c & -0.03 & 5.6 & P2020: unres. [IC2014: 0.120 317.6] \\
\hline $00055+3406$ & HU1201 & 2012.854 & 0.158 & 312.7 & Zir2003 & -0.02 & 5.7 & P2014: 0.147310 .6 (O-C: $-0.013 .8)$ \\
\hline $00324+0657$ & $\mathrm{MCA} 1 \mathrm{Aa}, \mathrm{Ab}$ & 2012.879 & 0.145 & 273.6 & Msn2012a & 0.03 & 10.1 & P2020: unres. [IC2011: 0.153 269.2] \\
\hline $00324+0657$ & $\mathrm{MCA} 1 \mathrm{Aa}, \mathrm{Ab}$ & 2012.999 & 0.108 & 273.8 & Msn2012a & -0.01 & 11.0 & P2020: unres. [IC2011: 0.153 269.2] \\
\hline $00516+2237$ & A1808 & 2011.582 & 0.151 & 197.9 & Doc2005b & -0.05 & -3.9 & P2020: 0.224204 .6 (O-C: $0.01-2.4)$ \\
\hline $00533+0405$ & A2307 & 2012.980 & 0.131 & 217.5 & Tok2015c & -0.00 & -6.5 & P2020: unres. [IC2014: 0.117 232.9] \\
\hline $01108+6747$ & HDS155 & 2012.988 & 0.154 & 165.3 & Doc2018i & 0.03 & -0.7 & [IC2012: 0.121 163.6] \\
\hline $01277+0521$ & BU1164AB & 2012.999 & 0.156 & 172.7 & Doc2010i & -0.01 & -9.1 & P2020: 0.386166 .9 (O-C: $0.01-0.2)$ \\
\hline $02157+2503$ & COU79 & 2011.992 & 0.125 & 42.0 & Mut2010e & -0.04 & -8.5 & P2020: 0.126357 .9 (O-C: 0.02 0.9) \\
\hline $02512+0141$ & A2338 & 2011.066 & 0.150 & 355.9 & Doc $2016 a$ & 0.03 & 7.3 & P2020: 0.23713 .2 (O-C: $0.04-18.6)$ \\
\hline $03035+2304$ & HDS389 & 2012.999 & 0.158 & 351.9 & Bag2005 & 0.02 & -9.0 & P2020: 0.1724 .0 (O-C: $-0.00-6.6)$ \\
\hline $07336+1550$ & MCA32 & 2012.238 & 0.147 & 111.7 & Zir2008 & -0.05 & 4.3 & P2020: unres. [IC2004: 0.20 105.8] \\
\hline $09123+1500$ & FIN347Aa, Ab & 2011.135 & 0.118 & 141.8 & Msn2018b & -0.04 & 0.6 & P2020: unres. [IC2014: 0.148 0.128.4] \\
\hline $14138+3059$ & COU606 & 2011.387 & 0.145 & 220.5 & Doc2008a & 0.02 & 9.6 & P2013: 0.227345 .2 (O-C: $0.11-8.0)$ \\
\hline $14138+3059$ & COU606 & 2012.339 & 0.082 & 181.8 & Doc2008a & -0.04 & -5.0 & P2013: 0.227345 .2 (O-C: $0.11-8.0)$ \\
\hline $14260+4213$ & COU1757 & 2011.387 & 0.114 & 54.7 & Hrt2014b & -0.01 & 5.3 & P2020: 0.184117 .8 (O-C: $-0.01-6.3)$ \\
\hline $14260+4213$ & COU1757 & 2012.359 & 0.134 & 73.5 & Hrt2014b & 0.01 & 12.6 & P2020: 0.184117 .8 (O-C: $-0.01-6.3)$ \\
\hline $14260+4213$ & COU1757 & 2012.362 & 0.087 & 71.0 & Hrt2014b & -0.04 & 10.1 & P2020: 0.184117 .8 (O-C: $-0.02-6.3)$ \\
\hline $14558+3939$ & A1627 & 2011.384 & 0.159 & 8.0 & Baz1993a & -0.06 & 2.3 & P2020: $0.221205 .5(\mathrm{O}-\mathrm{C}:-0.023 .5)$ \\
\hline $14588+3551$ & COU1136 & 2012.359 & 0.143 & 148.4 & Gur2020 & 0.10 & -11.3 & [IC2008: 0.085 64.3] \\
\hline $16584+3943$ & COU1289 & 2012.479 & 0.131 & 63.1 & Doc2013e & 0.02 & -3.3 & [IC2010: 0.123 71.1] \\
\hline $17080+3556$ & HU1176 & 2012.517 & 0.114 & 98.1 & Mut2010b & 0.01 & 12.5 & [IC2011: 0.124 97.9] \\
\hline $17155+1052$ & HDS2440 & 2011.526 & 0.131 & 192.9 & Cve2014 & 0.03 & 2.9 & [IC2010: 0.109 206.3) \\
\hline $17161+2316$ & COU315 & 2011.455 & 0.148 & 135.6 & Doc $2020 \mathrm{~b}$ & 0.07 & 14.7 & P2016: 0.141112 .9 (O-C: 0.02 35.3) \\
\hline $17161+2316$ & COU315 & 2012.518 & 0.121 & 128.2 & Doc $2020 b$ & 0.05 & 17.8 & P2016: 0.141112 .9 (O-C: 0.02 35.3) \\
\hline $17313+1901$ & COU499 & 2012.479 & 0.133 & 348.9 & Tok2017c & 0.04 & 1.1 & [IC2015: 0.0927 333.0] \\
\hline $17326+3445$ & HU1181 & 2012.518 & 0.150 & 316.7 & Sod1999 & 0.05 & -2.2 & [IC2012: 0.112 323.0] \\
\hline $17418+2130$ & COU114 & 2012.518 & 0.096 & 261.1 & Cou1999b & -0.01 & -8.4 & P2015: 0.188130 .1 (O-C: 0.09 22.0) \\
\hline $18410+2450$ & A2988 & 2012.556 & 0.135 & 220.4 & FMR2010f & 0.01 & -8.6 & P2015: 0.142221 .6 (O-C: 0.00 0.2) \\
\hline $18466+3821$ & HU1191 & 2011.573 & 0.138 & 121.4 & Doc2009g & 0.04 & 7.0 & P2015: 0.177344 .6 (O-C: $-0.03-10.3)$ \\
\hline $19027+4307$ & YSC13 & 2012.583 & 0.097 & 167.0 & Doc2013j & 0.01 & -7.8 & [IC2012: 0.083 174.6] \\
\hline $19069+4137$ & COU2197 & 2011.559 & 0.136 & $149.3^{*}$ & Doc $2016 g$ & -0.05 & $14.1^{Q}$ & {$[$ IC2008: $0.211: 168]$} \\
\hline $19069+4137$ & COU2197 & 2012.627 & 0.148 & $146.8^{*}$ & Doc2016g & -0.03 & $20.3^{Q}$ & [IC2008: $0.211: 168]$ \\
\hline $19302+5639$ & A712 & 2011.516 & 0.107 & 72.2 & USN2006b & 0.01 & -1.6 & [IC2009: 0.16 93.] \\
\hline $19350+2328$ & A162 & 2012.635 & 0.153 & 8.0 & Mdz2017 & 0.00 & 9.5 & [IC2015: 0.143 114.8] \\
\hline $19411+1349$ & KUI93 & 2011.510 & 0.141 & 319.5 & Tok2021 & -0.03 & 5.0 & P2020: 0.150318 .4 (O-C: 0.04 0.2) \\
\hline $19550+4152$ & HO581 & 2012.712 & 0.115 & 229.7 & Sod1999 & -0.04 & 3.1 & [IC2006: 0.286 312.8] \\
\hline $20311+1548$ & A1675 & 2011.808 & 0.105 & 324.5 & Baz1987d & -0.09 & 7.6 & [IC2015: 0.151 306.4] \\
\hline $21001+0731$ & KUI102 & 2012.816 & 0.135 & 287.4 & Hrt2014b & -0.01 & -0.6 & P2020: 0.234192 .4 (O-C: $0.01-1.6)$ \\
\hline $21135+1559$ & HU767 & 2011.748 & 0.086 & 165.3 & Hrt1996a & 0.05 & 89.8 & [IC2008: 0.154 171.0] \\
\hline $21135+1559$ & HU767 & 2011.827 & 0.114 & 170.6 & Hrt1996a & 0.08 & 95.0 & [IC2008: 0.154 171.0] \\
\hline $21423+0555$ & HU280 & 2012.870 & 0.152 & 184.7 & USN2006a & -0.01 & $-3.1^{Q}$ & P2020: unres. [IC2014: 0.121 193.1] \\
\hline $21510+2911$ & A 889 & 2011.726 & 0.156 & 126.1 & Hei1984b & 0.05 & 5.9 & P2020: 0.2064 .5 (O-C: 0.0717 .0 \\
\hline $21539+2821$ & WSI8 & 2011.740 & 0.111 & 142.2 & HIP1997d & 0.02 & 2.5 & P2020: unres. [IC2007: $0.115: 78.7]$ \\
\hline $21573+3241$ & A1226 & 2011.740 & 0.103 & $180.9^{\prime}$ & Cou $1987 \mathrm{c}$ & -0.09 & $-3.9^{Q}$ & [IC1996: 0.132 215.5] \\
\hline $21593+4606$ & COU2138 & 2012.764 & 0.129 & 145.3 & Doc2012c & -0.01 & 8.9 & P2020: 0.16236 .0 (O-C: -0.02 2.0) \\
\hline $22029+1547$ & HDS3129 & 2011.827 & 0.154 & 158.4 & Tok2015c & 0.06 & -1.2 & P2020: unres. [IC2014: 0.100 140.6] \\
\hline $22045+1551$ & BU696 & 2012.870 & 0.156 & 356.8 & FMR2013d & -0.04 & 4.3 & P2020: 0.333354 .7 (O-C: 0.000 .7 ) \\
\hline $22384+2943$ & $\mathrm{HO} 480$ & 2012.854 & 0.131 & 317.8 & FMR2014b & 0.02 & -11.8 & P2020: unres. [IC1991: 0.323 255.0] \\
\hline $22408-0333$ & KUI114 & 2011.754 & 0.116 & 130.2 & Doc2016d & 0.02 & 14.5 & P2020: 0.383128 .7 (O-C: 0.02 0.7) \\
\hline $22474+1749$ & WSI93 & 2012.854 & 0.159 & 138.2 & Tok2017c & -0.08 & -1.2 & P2020: unres. [IC2014: 0.187 162.0] \\
\hline $22474+1749$ & WSI93 & 2012.870 & 0.137 & 141.6 & Tok2017c & -0.10 & 2.0 & P2020: unres. [IC2014: 0.187 162.0] \\
\hline $22546+1054$ & HDS3257 & 2012.816 & 0.131 & 67.3 & Tok2015c & -0.03 & -3.8 & P2020: unres. [IC2014: 0.145 75.1] \\
\hline $22570+2441$ & COU542Aa,Ab & 2012.854 & 0.128 & 85.4 & Doc2016b & -0.04 & 3.8 & P2020: unres. [IC2008: 0.239 45.7] \\
\hline $23020+4800$ & A194 & 2012.868 & 0.130 & 287.3 & Doc2017e & 0.02 & 7.0 & P2015: 0.113289 .1 (O-C: 0.04 13.2) \\
\hline
\end{tabular}



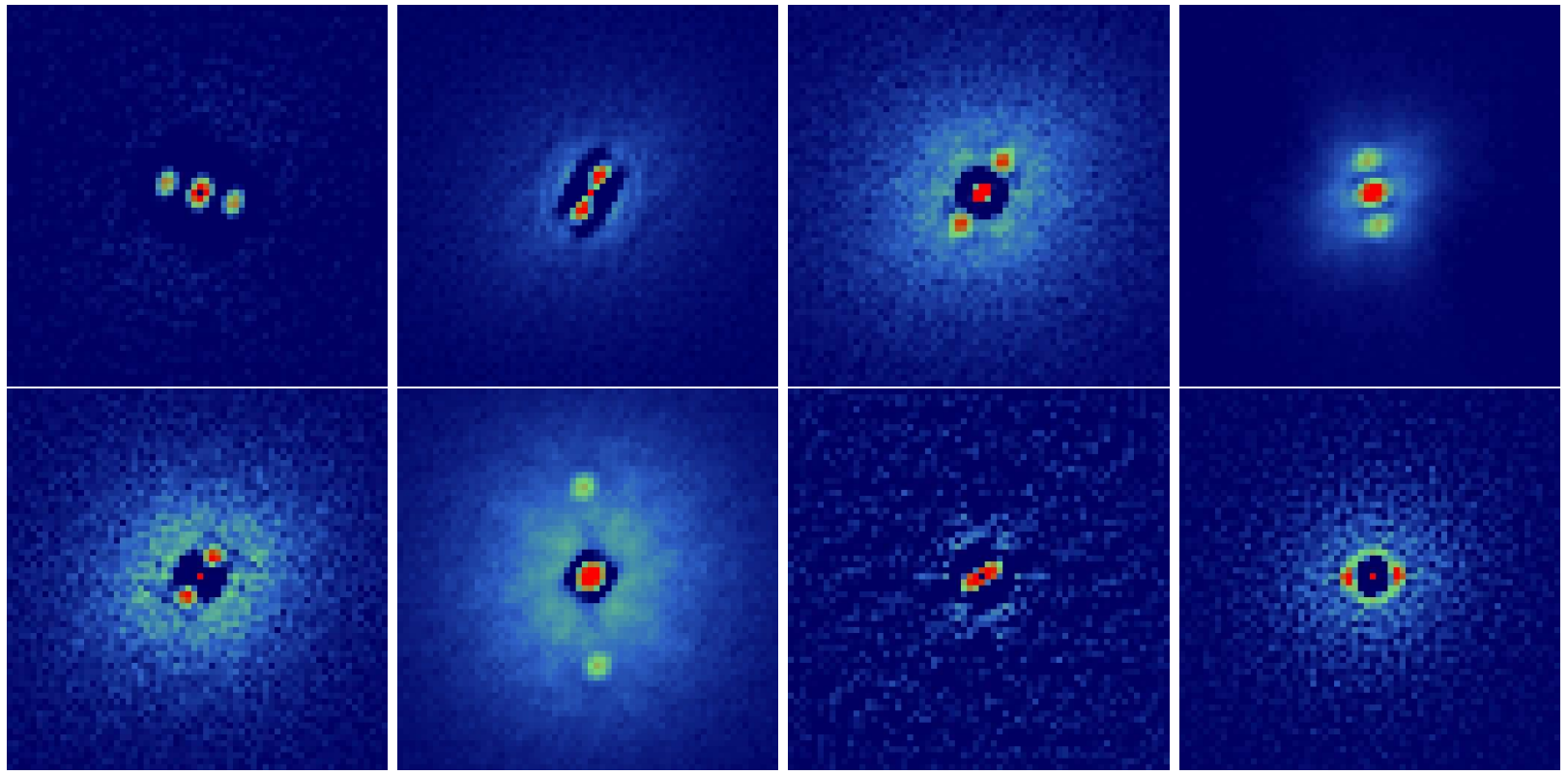

Fig. 8 Autocorrelations of some binaries with large residuals from Table 5. From left to right and top to bottom: A1247, BU367, COU1006, COU1271, COU2197, HO303, HU374, and MLR182Aa,Ab (2011). North direction is to the bottom and East to the right.
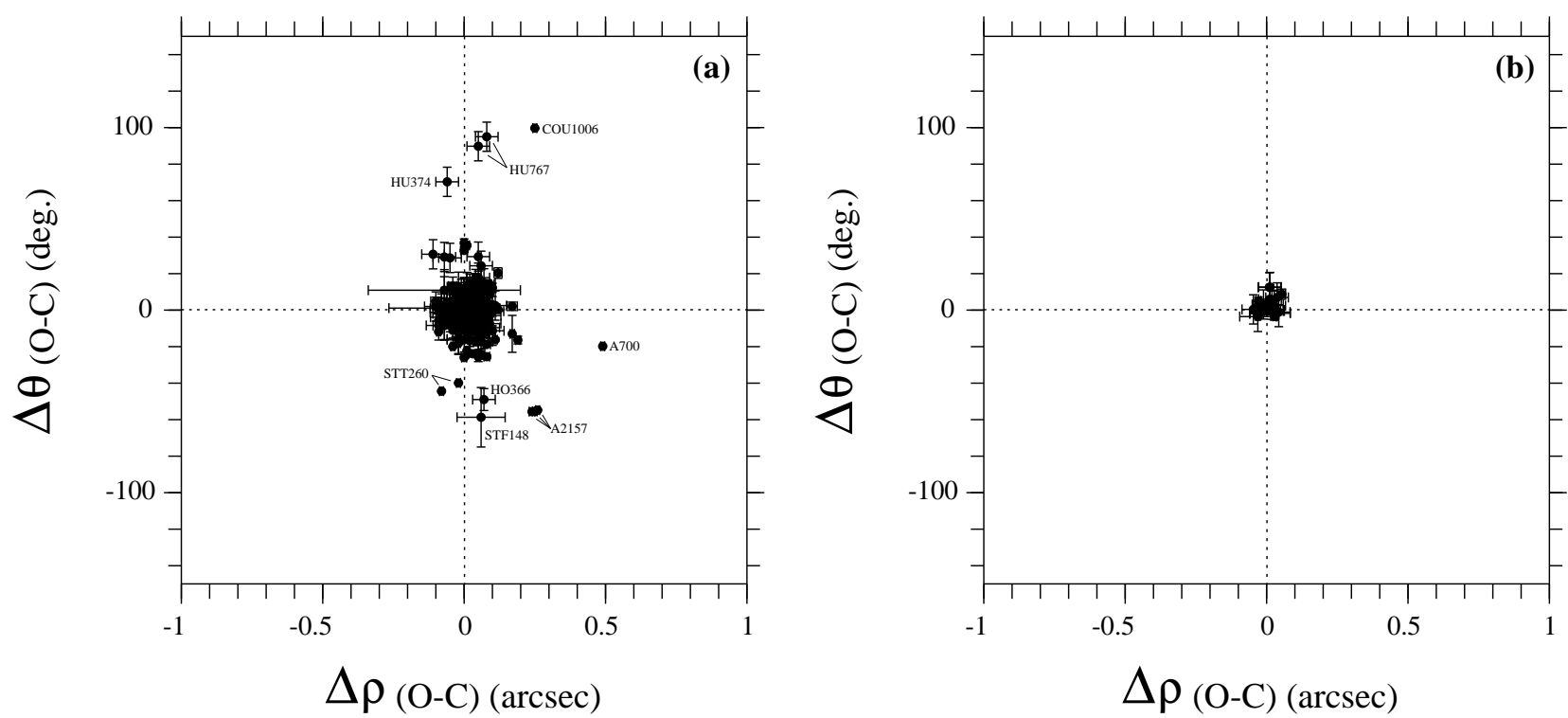

Fig. 9 Residuals from published orbits of our measurements reported in Table 7: (a) with all orbits, and (b) with the best orbits only (i.e., of Grade 1 in OC6).

monitored until now. By using the Direct Vector Autocorrelation method, we have measured the difference of magnitude of the two components for 326 objects with an estimated error of 0.2 mag. Thanks to good seeing images and with the use of high-contrast numerical filters, we have also been able to obtain 196 measurements with an angular separation smaller than the diffraction limit of our instrumentation, and consis- tent with those obtained with larger telescopes. During good seeing conditions, we obtained new measurements of the 15th magnitude faint multiple system CON37 with the Lucky-imaging technique. We also found a new binary close to that system. As a result, we report 47 measurements of 28 new binaries that we have found during our observations in 2011-2012. 


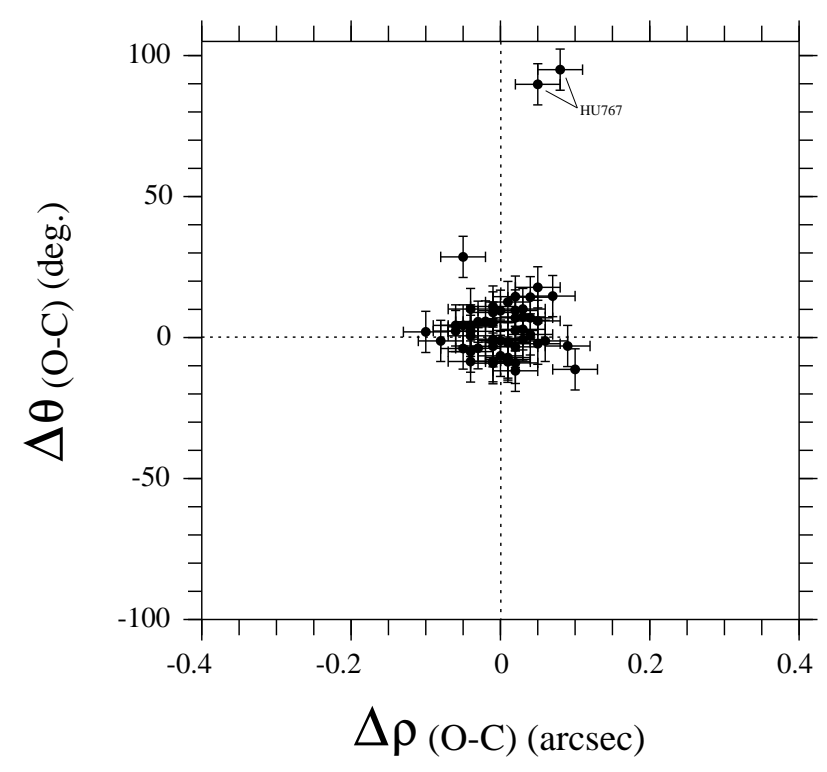

Fig. 10 Residuals from published orbits of our measurements with angular separations smaller than the diffraction limit (from Table 6, with all orbits).

This work is thus a good contribution to the continuing monitoring of long period visual binary systems, which is important for refining systemic stellar masses. It validates the technical and data processing investment that we have made in the preceding years. This work is fully integrated in the observation efforts of the Commission G1, "Binary and Multiple Star Systems" of the International Astronomical Union. Our measurements will be added to the worldwide database of this Commission G1, which is currently maintained by the U.S. Naval Observatory.

Acknowledgements. We are indebted to the directors of the Observatoire de la Côte d'Azur for allowing us to use the 76-cm refractor and to the staff from this Observatory for their technical support. In particular, we thank Guy Morlet for writing a program dedicated to the $76-\mathrm{cm}$ refractor, that was used for pointing stars with the telescope. We are grateful to F. Losse for adapting the REDUC software for our needs, and to C. Buil for providing his IRIS software to the amateur community.

This work has made use of the "Fourth Catalogue of Interferometric Measurements of Binary Stars"

(http://www.astro.gsu.edu/wds/int4.html), the "Sixth Catalogue of Orbits of Visual Binary Stars"

(http://www.astro.gsu.edu/wds/orb6.html), the Washington Double Star Catalogue maintained at the U.S. Naval Observatory, (http://www.astro.gsu.edu/wds), the SIDONIe (Site Informatique des étoiles DOubles de Nice) http://sidonie.obs-nice.fr and the SIMBAD data base (http://simbad.u-strasbg.fr/simbad) operated by the Centre de Données Astronomiques de Strasbourg (France). This research has made use of "Aladin sky atlas" developed at CDS, Strasbourg Observatory, France.

\section{References}

ANDOR, 2021, ANDOR technology

http://www.andor-tech.com

Bagnuolo, W.G., Mason, B.D., Barry, D.J., Hartkopf, W.I., McAlister, H.A., 1992, AJ, 103, 1399

Bonnarel, F., Fernique, P., Bienaymé, O., Egret, D., Genova, F., Louys, M., Ochsenbein, F., Wenger, M., Bartlett, J. G., Aladin Sky Atlas, http://aladin.ustrasbg.fr, 2000 A\&AS 143, 33

Buil, C., 2021, IRIS software http://www.astrosurf.com/buil/us/iris/iris.htm

Connelley, M.S., Reipurth, B., Tokunaga, A.T., 2008, AJ, 135,2496

Couteau, P., 1999, Catalogue de 2700 Etoiles Doubles "COU", Publ. Obs. Côte d'Azur, Nice, France

Gili, R., Prieur, J.-L., 2012, Astron. Nach., 333, 727-735 (Paper I)

Gili, R., Prieur, J.-L., Rivet, J.-P., Vakili, F., Koechlin, L., Bonneau, D., 2014, The Observatory, 134, 267

Gili, R., 2016, "New double stars discovered by R. Gili with PISCO2", IAU Commission G1 - Double Stars Inf. Circ. 190, 4

Gili, R., Prieur, J.-L., Rivet, J.-P., Scardia, M., Pansechi, L., Argyle, R.W., Ling, J.F., Piccotti, L., Aristidi, E., Koechlin, L., Bonneau, D., Maccarini, L., 2020, Astron. Nach., 341, 441 (Paper II)

Hartkopf, W.I., Mason, B.D., Worley, C.E., 2001, "The 2001 US Naval Observatory Double Star CD-ROM. II. The Fifth Catalog of Orbits of Visual Binary Stars"

Matson, R.A., Williams, S.J, Hartkopf, W.I., Mason, B.D., 2021, "Sixth Catalog of Orbits of Visual Binary Stars" http://http://www.astro.gsu.edu/wds/orb6.html (OC6)

Hartkopf, W.I., Mason, B.D., Wycoff, G.L., McAlister, H.A., 2021, "Fourth Catalog of Interferometric Measurements of Binary Stars" http://www.astro.gsu.edu/wds/int4.html (IC4)

Heintz, W.D. 1998, ApJS 117, 587

Labeyrie, A., 1970, A\&A, 6, 85

Le Contel, D., Valtier, J.-C., Bonneau, D., 2001, A\&A, 377, 496, Site Informatique des étoiles DOubles de Nice (SIDONIe) http://sidonie.obs-nice.fr

Losse, F., 2021, REDUC, data processing software http://www.astrosurf.com/hfosaf/

Mason, B.D., Wycoff, G.L., Hartkopf, W.I., 2001, "Washington Double Star Catalog", AJ, 122, 3466, and http://www.astro.gsu.edu/wds/ (WDS)

Parent, I.R., Vaughn, S.T., Conover, E.D., Williams, M., Steed, J., Orman, D., Gardella, J.C., Siverson, N., Ogden, J. \& Genet, R., 2017, JDSO 13, 384

Prieur, J.-L, Koechlin, L., André, C., Gallou, G., Lucuix, C., 1998, Experimental Astronomy, vol 8, Issue 4, 297

Prieur, J.-L., Oblak, E., Lampens, P., Kurpinska-Winiarska, M., Aristidi, E., Koechlin, L., Ruymaekers, G., 2001, A\&A, 367, 865-875

Scardia, M., Prieur, J.-L., Pansecchi, L., Argyle, R.W., Basso, S., Sala, M., Ghigo, M., Koechlin, L., Aristidi, E., 2007, MNRAS, 374, 965-978

Scardia, M., Prieur, J.-L., Pansecchi, L., Argyle, R.W., Sala, M., 2011, Astron. Nach., 332, 508

Scardia, M., Rivet, J.-P., Prieur, J.-L., Pansechi, L., Argyle, R.W., Ling, J., Aristidi, E., Zanutta, A., Vernet, 

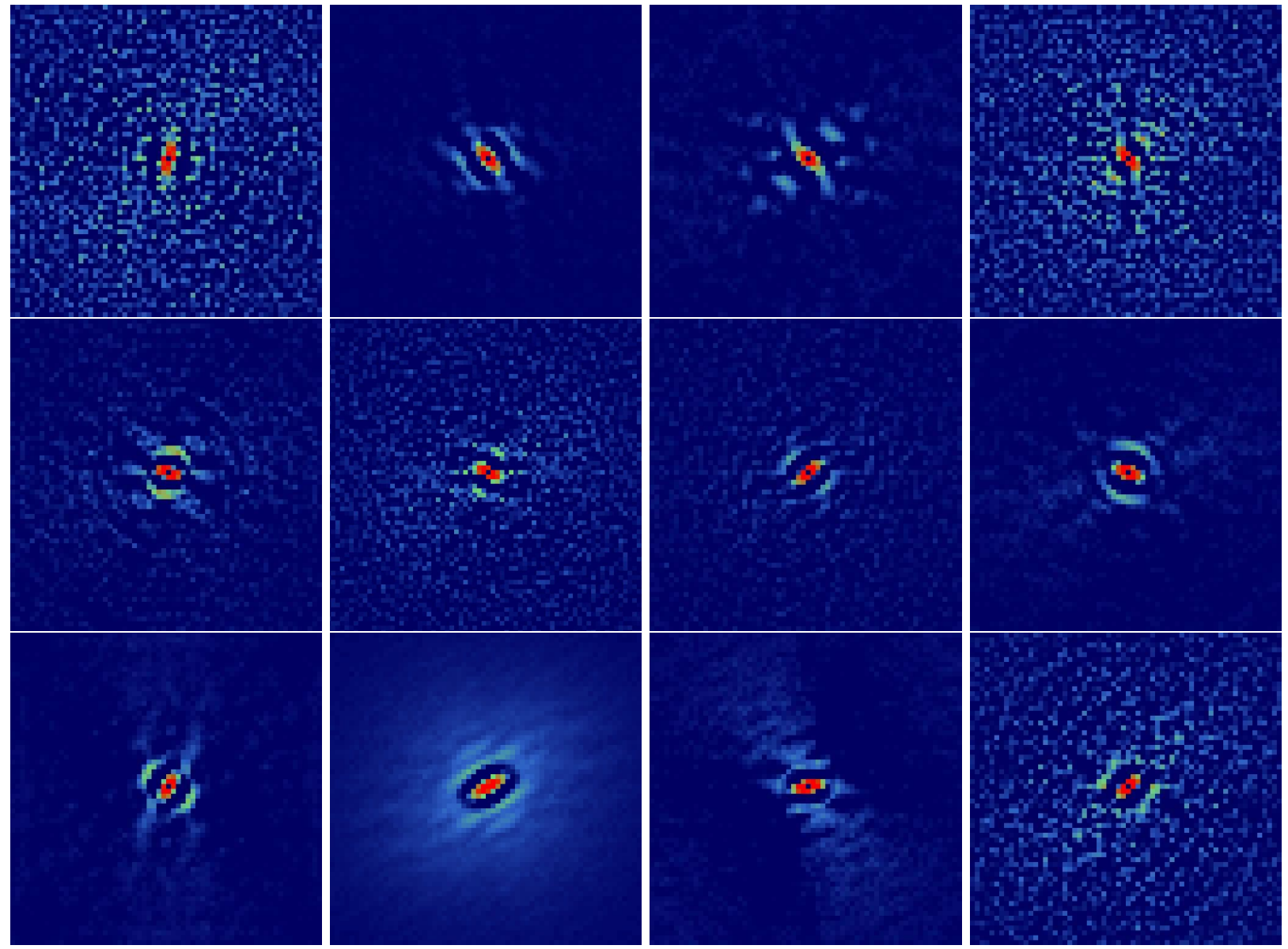

Fig. 11 Autocorrelations of some binaries with separations closer than the diffraction limit from Table 6. From left to right and top to bottom: A428, COU79, COU114, COU606, COU1289, COU1757, HO480, HO581, HU767, HU1134, HU1176 (2011), and WSI8. North direction is to the bottom and East to the right.

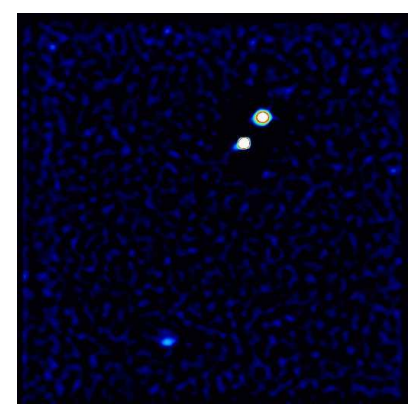

$19247+2238$

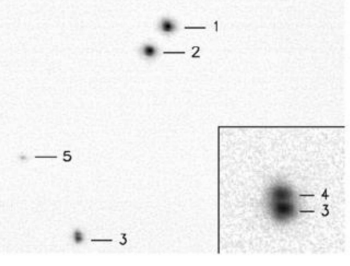

Fig. 12 Lucky image obtained with PISCO2 of the multiple system CON37 (left) compared to the $20^{\prime \prime} \times 20^{\prime \prime} L^{\prime}$ field centered on IRAS $19247+2238$ published by Connelley et al., 2008 (right). North direction is to the top and East to the left.

D., Abe, L., Bendjoya, P., Dimur, C., Suarez, O. 2019, Astron. Nachr., 340, 771

SIMBAD data base from the CDS (Centre de Données Stellaires de Strasbourg), 2021, http://simbad.u-strasbg.fr/simbad (CDS)

Tokovinin, A.A., 2021, IAUDS Commission G1 Inf. Circ. 203,1

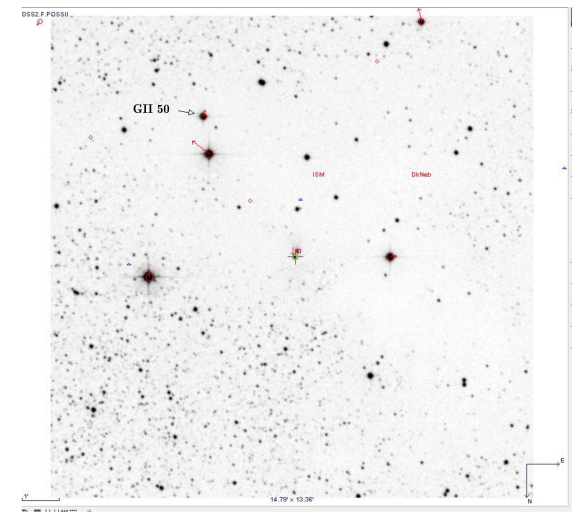

Fig. 13 Location of the new double star GII 50 that was discovered in the field of CON37AB. This image was produced by the Aladin software (Bonnarel et al., 2000) with DDS2 (centered on IRAS $19247+2238$, with a field of view of 14.79 'x13.36'). North direction is to the bottom and East to the right.

Worden, S.P., Murray, K.S., Schmidt, G.D., Angel, J.R.P., 1977, Icarus 32,450 
Table 7 Measurements of multiple stars obtained with PISCO2 in 2011-2012

\begin{tabular}{|c|c|c|c|c|c|c|c|c|c|c|c|c|}
\hline WDS & Name & Epoch & Bin. & $\begin{array}{c}\rho \\
(")\end{array}$ & $\begin{array}{l}\sigma_{\rho} \\
(")\end{array}$ & $\begin{array}{c}\theta \\
\left({ }^{\circ}\right)\end{array}$ & $\begin{array}{l}\sigma_{\theta} \\
\left(^{\circ}\right)\end{array}$ & $\Delta m$ & Notes & Orbit & $\begin{array}{r}\Delta \rho(\mathrm{O}-\mathrm{C}) \\
(")\end{array}$ & $\begin{array}{r}\Delta \theta(\mathrm{O}-\mathrm{C}) \\
\left({ }^{\circ}\right)\end{array}$ \\
\hline $00003+6557$ & MLR280 & 2012.988 & 1 & 1.512 & 0.021 & $9.6^{*}$ & 0.3 & & & & & \\
\hline $00004+2749$ & TDS1238 & 2012.840 & 1 & 0.818 & 0.007 & $86.4^{*}$ & 0.3 & & & & & \\
\hline $00008+1659$ & BAG18 & 2012.840 & 1 & 0.674 & 0.007 & 177.5 & 0.4 & & & Tok2019c & 0.00 & 4.7 \\
\hline $00014+3937$ & HLD60 & 2011.582 & 1 & 1.324 & 0.007 & $168.9^{*}$ & 0.3 & & & Izm2019 & 0.02 & 0.5 \\
\hline $00022+2705$ & BU733AB & 2011.582 & 1 & 0.782 & 0.037 & 286.8 & 0.3 & & & Sod1999 & 0.02 & -0.5 \\
\hline $00022+2705$ & BU733AB & 2011.830 & 1 & 0.718 & 0.037 & $287.8^{*}$ & ${ }^{*} 0.3$ & & & Sod1999 & -0.02 & -2.0 \\
\hline $00022+2705$ & BU733AB & 2011.830 & 1 & 0.720 & 0.037 & 288.0 & 0.3 & & & Sod1999 & -0.02 & -1.8 \\
\hline $00022+2705$ & BU733AB & 2012.854 & 1 & 0.667 & 0.057 & 300.3 & 0.3 & & & Sod1999 & 0.03 & -1.8 \\
\hline $00024+1047$ & A1249 & 2011.751 & 1 & 0.235 & 0.011 & $257.3^{*}$ & ${ }^{*} 1.8$ & & & Zir2003 & 0.06 & 4.7 \\
\hline $00026-0829$ & $\mathrm{~A} 428$ & 2011.751 & 1 & 0.107 & 0.030 & 337.8 & 3.5 & & & Tok2015c & -0.03 & 5.6 \\
\hline $00026+1841$ & $\mathrm{HDS} 2 \mathrm{Aa}, \mathrm{Ab}$ & 2011.830 & 1 & 0.156 & 0.040 & 150.5 & 8.0 & & & & & \\
\hline $00026+1841$ & $\mathrm{HDS} 2 \mathrm{Aa}, \mathrm{Ab}$ & 2012.840 & 1 & 0.156 & 0.040 & 144.4 & 8.0 & & & & & \\
\hline $00026+1841$ & $\mathrm{HDS} 2 \mathrm{Aa}, \mathrm{Ab}$ & 2012.854 & 1 & 0.126 & 0.040 & 149.7 & 8.0 & & & & & \\
\hline $00026+1841$ & $\mathrm{HDS} 2 \mathrm{AB}$ & 2011.830 & 1 & 2.015 & 0.034 & 337.3 & 1.3 & & & & & \\
\hline $00026+1841$ & HDS2AB & 2012.854 & 1 & 2.026 & 0.030 & 337.4 & 1.3 & & & & & \\
\hline $00026+1841$ & HDS2AB & 2012.854 & 1 & 2.072 & 0.040 & $336.6^{*}$ & ${ }^{*} 1.3$ & 4.69 & & & & \\
\hline $00039+2759$ & $\mathrm{~A} 429 \mathrm{AB}$ & 2011.582 & 1 & 0.542 & 0.037 & $331.4^{*}$ & ${ }^{*} 0.3$ & & & & & \\
\hline $00039+2759$ & HJ1929AB,C & 2011.582 & 1 & 4.684 & 0.023 & $249.9^{*}$ & 0.3 & & & & & \\
\hline $00047+3547$ & HU800 & 2011.759 & 1 & 0.204 & 0.013 & $246.6^{*}$ & ${ }^{*} 1.0$ & & & & & \\
\hline $00054+3348$ & COU646 & 2012.840 & 1 & 0.468 & 0.027 & $150.8^{*}$ & ${ }^{*} 0.3$ & & & & & \\
\hline $00055+3406$ & HU1201 & 2011.582 & 1 & 0.185 & 0.040 & $305.6^{*}$ & 8.0 & & & Zir2003 & -0.00 & -1.5 \\
\hline $00055+3406$ & HU1201 & 2012.854 & 1 & 0.158 & 0.040 & 312.7 & 8.0 & & & Zir2003 & -0.02 & 5.7 \\
\hline $00058+2855$ & HEI831 & 2011.584 & 1 & 1.897 & 0.041 & $136.4^{*}$ & ${ }^{*} 0.4$ & & & & & \\
\hline $00068+0427$ & BU1155 & 2012.980 & 1 & 0.662 & 0.007 & $75.9^{*}$ & 0.4 & & & & & \\
\hline $00073+0742$ & HDS13 & 2012.980 & 1 & 0.376 & 0.007 & $141.6^{*}$ & * 0.3 & & & & & \\
\hline $00073+2058$ & HDS12 & 2012.840 & 1 & 1.872 & 0.009 & $7.4^{*}$ & 0.3 & & & & & \\
\hline $00089+1332$ & TDS1313 & 2012.980 & 1 & 0.810 & 0.015 & $121.9^{*}$ & ${ }^{*} 0.8$ & & & & & \\
\hline $00092+0937$ & TDS1320 & 2012.980 & 1 & 0.596 & 0.007 & $217.6^{*}$ & 0.5 & & & & & \\
\hline
\end{tabular}




\begin{tabular}{|c|c|c|c|c|c|c|c|c|c|c|c|c|}
\hline WDS & Name & Epoch & Bin. & $\begin{array}{c}\rho \\
(")\end{array}$ & $\begin{array}{l}\sigma_{\rho} \\
(")\end{array}$ & $\begin{array}{c}\theta \\
\left({ }^{\circ}\right)\end{array}$ & $\begin{array}{l}\sigma_{\theta} \\
\left(^{\circ}\right)\end{array}$ & $\Delta m$ & Notes & Orbit & $\begin{array}{r}\Delta \rho(\mathrm{O}-\mathrm{C}) \\
(")\end{array}$ & $\begin{array}{r}\Delta \theta(\mathrm{O}-\mathrm{C}) \\
\left({ }^{\circ}\right)\end{array}$ \\
\hline $00093+7943$ & STF2 & 2012.988 & 1 & 0.900 & 0.007 & $17.4^{*}$ & 0.3 & & & Hei1997 & 0.01 & 1.6 \\
\hline $00095+1907$ & COU247 & 2011.582 & 1 & 0.313 & 0.015 & $233.3^{*}$ & 1.7 & & & Doc2012i & -0.01 & -9.9 \\
\hline $00095+1907$ & COU247 & 2012.840 & 1 & 0.280 & 0.007 & $232.8^{*}$ & ${ }^{k} 0.6$ & & & Doc2012i & -0.06 & -5.1 \\
\hline $00100+0835$ & A1801 & 2012.980 & 1 & 0.269 & 0.007 & $201.2^{*}$ & 2.2 & & & & & \\
\hline $00101+3825$ & HDS23Da,Db & 2011.584 & 1 & - & - & - & - & & NR & & & \\
\hline $00101+3825$ & $\mathrm{HDS} 23 \mathrm{Da}, \mathrm{Db}$ & 2011.830 & 1 & 0.219 & 0.10 & 146.1 & 2.7 & & & Cve2017b & 0.09 & 2.5 \\
\hline $00101+3825$ & SKF495DB & 2011.830 & 1 & 4.377 & 0.027 & $296.8^{*}$ & 0.3 & & & & & \\
\hline $00110+2908$ & COU346 & 2011.584 & 1 & 0.225 & 0.021 & $305.8^{*}$ & 2.1 & & & & & \\
\hline $00126+2143$ & BAG19 & 2012.873 & 1 & - & - & - & - & & NR & & & \\
\hline $00132+1144$ & HDS30 & 2012.920 & 1 & 0.317 & 0.007 & $260.5^{*}$ & 0.8 & & & & & \\
\hline $00134+2659$ & STT2 & 2011.582 & 1 & 0.445 & 0.009 & $160.8^{*}$ & 1.0 & & & Hrt2008 & 0.04 & 2.5 \\
\hline $00134+2659$ & STT2 & 2012.840 & 1 & 0.425 & 0.007 & $160.0^{*}$ & 0.3 & & & Hrt2008 & 0.02 & 2.7 \\
\hline $00137+1838$ & A2201 & 2012.879 & 1 & 1.178 & 0.007 & 199.1 & 0.3 & 3.13 & & & & \\
\hline $00137+1838$ & A2201 & 2012.879 & 1 & 1.202 & 0.007 & $199.3^{*}$ & 0.3 & & & & & \\
\hline $00138+7233$ & MLR282 & 2012.988 & 1 & 0.436 & 0.007 & $166.7^{*}$ & ${ }^{k} 0.5$ & & & & & \\
\hline $00145+1638$ & YSC78 & 2011.879 & 1 & 0.694 & 0.012 & $44.6^{*}$ & 0.3 & & & & & \\
\hline $00145+1638$ & YSC78 & 2012.841 & 1 & 0.658 & 0.007 & $42.4^{*}$ & 0.6 & & & & & \\
\hline $00149+2133$ & BU1027 & 2012.879 & 1 & 1.700 & 0.010 & $12.0^{*}$ & 0.3 & & & & & \\
\hline $00151+1907$ & A2202 & 2012.999 & 1 & 0.613 & 0.007 & $153.8^{*}$ & 0.3 & & & & & \\
\hline $00151+7854$ & MLR301 & 2012.988 & 1 & 0.787 & 0.086 & $229.5^{*}$ & 0.3 & & & & & \\
\hline $00153+4412$ & $\mathrm{~A} 1256 \mathrm{AB}$ & 2011.582 & 1 & - & - & - & - & & NR & & & \\
\hline $00156+3751$ & COU848 & 2011.582 & 1 & 0.779 & 0.007 & $297.9^{*}$ & ${ }^{k} 0.4$ & & & & & \\
\hline $00159+2103$ & TDS1373 & 2011.879 & 1 & 0.777 & 0.007 & $59.4^{*}$ & 0.3 & 0.10 & & & & \\
\hline $00162+3331$ & COU651 & 2012.841 & 1 & 0.373 & 0.010 & $109.2^{*}$ & 0.5 & & & & & \\
\hline $00162+3331$ & COU651 & 2011.828 & 1 & 0.310 & 0.011 & $111.5^{*}$ & 0.8 & 0.20 & & & & \\
\hline $00167+3629$ & STT4 & 2011.582 & 1 & 0.180 & 0.040 & $70.2^{*}$ & 8.0 & & & Sca2000b & -0.01 & -7.2 \\
\hline $00174+1002$ & A1804 & 2011.584 & 1 & 1.275 & 0.022 & $25.0^{*}$ & 0.3 & & & & & \\
\hline $00174+1852$ & HDS39 & 2011.830 & 1 & 1.522 & 0.010 & $248.7^{*}$ & 0.5 & & & & & \\
\hline $00176+3946$ & COU1048 & 2011.828 & 1 & 0.400 & 0.010 & $314.9^{*}$ & 0.6 & & & & & \\
\hline $00187+1559$ & STF25 & 2011.584 & 1 & 1.088 & 0.007 & $196.1^{*}$ & 0.3 & & & & & \\
\hline $00191+2822$ & TDS1397 & 2011.879 & 1 & - & - & - & - & & NR & & & \\
\hline $00206+1219$ & BU1015 & 2011.585 & 1 & 0.497 & 0.007 & $104.5^{*}$ & 0.6 & & & Hrt2010a & 0.01 & -0.7 \\
\hline $00206+1219$ & BU1015 & 2012.879 & 1 & 0.504 & 0.007 & $105.7^{*}$ & ${ }^{k} 0.4$ & & & Hrt2010a & 0.01 & -0.5 \\
\hline $00209+1059$ & BU1093 & 2012.980 & 1 & 0.770 & 0.007 & $118.1^{*}$ & ${ }^{k} 0.3$ & & & $\operatorname{Lin} 2010 c$ & 0.01 & -0.6 \\
\hline $00210+6740$ & HJ1018 & 2012.988 & 1 & 1.694 & 0.008 & $87.4^{*}$ & 0.3 & & & Sod1999 & -0.07 & -0.3 \\
\hline $00213+3553$ & COU747 & 2011.582 & 1 & 1.072 & 0.007 & $148.6^{*}$ & ${ }^{k} 0.3$ & & & & & \\
\hline $00220+4033$ & A1502 & 2012.868 & 1 & 0.945 & 0.007 & $244.0^{*}$ & 0.3 & & & & & \\
\hline $00221+6211$ & HDS49 & 2012.988 & 1 & 0.306 & 0.017 & $226.0^{*}$ & 2.2 & & & & & \\
\hline $00222+1114$ & HEI301 & 2012.980 & 1 & 1.705 & 0.009 & $280.5^{*}$ & 0.3 & & & & & \\
\hline $00233+0556$ & HDS53 & 2012.873 & 1 & 0.624 & 0.007 & $345.6^{*}$ & 0.5 & & & & & \\
\hline $00233+1645$ & HEI16 & 2012.879 & 1 & 0.258 & 0.007 & $19.2^{*}$ & 0.9 & & & & & \\
\hline $00233+1645$ & HEI16 & 2011.585 & 1 & 0.340 & 0.017 & $11.9^{*}$ & 2.5 & & & & & \\
\hline $00233+5132$ & TDS1431 & 2012.868 & 1 & 0.209 & 0.007 & $121.2^{*}$ & 1.2 & & & & & \\
\hline $00240+4912$ & HDS55 & 2012.868 & 1 & - & - & - & - & & NR & & & \\
\hline $00246+1659$ & YSC80 & 2012.841 & 1 & 0.889 & 0.047 & 208.8 & 0.3 & 4.06 & & & & \\
\hline $00246+1659$ & YSC80 & 2012.841 & 1 & 0.945 & 0.037 & $208.5^{*}$ & 0.7 & & & & & \\
\hline $00260+1905$ & HDS59 & 2012.999 & 1 & 0.808 & 0.007 & $264.2^{*}$ & 0.3 & & & & & \\
\hline $00263+6515$ & MLR80 & 2012.988 & 1 & 0.255 & 0.007 & $194.6^{*}$ & ${ }^{k} 0.9$ & & & & & \\
\hline $00271+1852$ & TDS19 & 2011.879 & 1 & 0.880 & 0.009 & $138.8^{*}$ & 0.3 & 0.40 & & & & \\
\hline $00272+2115$ & BU1225 & 2012.999 & 1 & 1.387 & 0.010 & $190.9^{*}$ & 0.3 & & & & & \\
\hline $00279+2334$ & BU779 & 2012.980 & 1 & 0.640 & 0.007 & $244.3^{*}$ & 0.4 & & & & & \\
\hline $00283+6344$ & HU1007 & 2012.988 & 1 & 0.392 & 0.007 & $97.8^{*}$ & 1.0 & & & Msn2017a & -0.02 & 3.4 \\
\hline $00284+0553$ & HEI610 & 2012.870 & 0 & 1.037 & 0.027 & 92.4 & 0.3 & & & & & \\
\hline
\end{tabular}




\begin{tabular}{|c|c|c|c|c|c|c|c|c|c|c|c|c|}
\hline WDS & Name & Epoch & Bin. & $\begin{array}{c}\rho \\
(")\end{array}$ & $\begin{array}{l}\sigma_{\rho} \\
(")\end{array}$ & $\begin{array}{c}\theta \\
\left({ }^{\circ}\right)\end{array}$ & $\begin{array}{l}\sigma_{\theta} \\
\left(^{\circ}\right)\end{array}$ & $\Delta m$ & Notes & Orbit & $\begin{array}{r}\Delta \rho(\mathrm{O}-\mathrm{C}) \\
(")\end{array}$ & $\begin{array}{r}\Delta \theta(\mathrm{O}-\mathrm{C}) \\
\left({ }^{\circ}\right)\end{array}$ \\
\hline $00284+0553$ & HEI610 & 2012.873 & 1 & 1.025 & 0.027 & $272.6^{*}$ & 0.3 & & & & & \\
\hline $00284+0553$ & HEI610 & 2012.879 & 1 & 1.037 & 0.027 & $271.6^{*}$ & 0.4 & & & & & \\
\hline $00290+4825$ & HDS64 & 2012.868 & 1 & 0.135 & 0.040 & 37.6 & 8.0 & & & & & \\
\hline $00298+3650$ & COU1049AB & 2011.582 & 1 & 0.749 & 0.007 & $37.9^{*}$ & 0.5 & & & & & \\
\hline $00298+3650$ & COU1049AC & 2011.582 & 1 & 2.869 & 0.014 & $332.0^{*}$ & 0.3 & & & & & \\
\hline $00307+1339$ & HDS66 & 2012.999 & 1 & 0.952 & 0.007 & $267.6^{*}$ & 1.6 & & & & & \\
\hline $00308+4732$ & BU394 & 2012.868 & 1 & 0.686 & 0.007 & $276.3^{*}$ & 0.3 & & & Zul1997b & -0.03 & -0.2 \\
\hline $00310+2839$ & CTU1 & 2011.879 & 1 & 0.884 & 0.007 & $224.1^{*}$ & 0.4 & 1.40 & & & & \\
\hline $00310+5015$ & HDS68 & 2012.868 & 1 & 0.389 & 0.007 & $354.2^{*}$ & 0.8 & & & & & \\
\hline $00317+1929$ & HDS70 & 2012.999 & 1 & 0.300 & 0.007 & $66.7^{*}$ & 0.8 & & & & & \\
\hline $00320+2740$ & COU547 & 2011.582 & 1 & - & - & - & - & & & NR & & \\
\hline $00324+0657$ & MCA1Aa,Ab & 2012.879 & 1 & 0.145 & 0.040 & 93.6 & 7.0 & & & Msn2012a & 0.03 & 10.1 \\
\hline $00324+0657$ & $\mathrm{MCA} 1 \mathrm{Aa}, \mathrm{Ab}$ & 2012.999 & 1 & 0.108 & 0.040 & 93.8 & 4.0 & & & Msn2012a & -0.01 & 11.0 \\
\hline $00324+2147$ & HDS72 & 2011.828 & 1 & 0.205 & 0.007 & 27.5 & 1.4 & & & & & \\
\hline $00326+0729$ & MCT1 & 2012.879 & 2 & 1.104 & 0.016 & 162.1 & 3.7 & & & & & \\
\hline $00331+1558$ & HEI17 & 2011.585 & 1 & 1.872 & 0.016 & $219.8^{*}$ & 0.7 & & & & & \\
\hline $00344+1844$ & COU250 & 2012.999 & 1 & 1.299 & 0.017 & $38.7^{*}$ & 0.3 & & & & & \\
\hline $00345+3015$ & COU654 & 2011.582 & 1 & 0.269 & 0.007 & $208.7^{*}$ & 0.3 & & & & & \\
\hline $00349+2627$ & COU548 & 2011.582 & 1 & 0.156 & 0.040 & 169.8 & 8.0 & & & & & \\
\hline $00352+1452$ & HLD61 & 2012.879 & 1 & 1.610 & 0.008 & $240.2^{*}$ & 0.3 & & & & & \\
\hline $00359+7549$ & HDS83 & 2012.988 & 1 & 0.315 & 0.008 & $199.3^{*}$ & 2.1 & & & & & \\
\hline $00360+3708$ & COU850 & 2011.585 & 1 & 0.396 & 0.007 & $314.4^{*}$ & 3.3 & & & & & \\
\hline $00360+3708$ & COU850 & 2011.828 & 1 & 0.367 & 0.007 & 317.3 & 4.5 & & & & & \\
\hline $00365+3724$ & A1505 & 2012.868 & 1 & 0.167 & 0.040 & $219.0^{*}$ & 8.0 & & & & & \\
\hline $00372+0806$ & FOX54 & 2012.879 & 1 & 1.494 & 0.007 & $153.4^{*}$ & 0.3 & & & & & \\
\hline $00374+3635$ & COU851 & 2011.585 & 1 & 0.401 & 0.007 & $213.4^{*}$ & * 0.3 & & & & & \\
\hline $00385+4022$ & COU1050 & 2011.582 & 1 & 0.636 & 0.007 & $93.1^{*}$ & 0.6 & & & & & \\
\hline $00390+4037$ & WOR31AB & 2011.585 & 1 & 0.260 & 0.037 & 124.4 & 0.9 & & & & & \\
\hline $00390+4037$ & WOR31AC & 2012.000 & 1 & 1.050 & 0.047 & 173.8 & 0.5 & & & & & \\
\hline $00390+4040$ & BU1159 & 2012.868 & 1 & 0.156 & 0.040 & $157.9^{*}$ & $* 8.0$ & & & & & \\
\hline $00395+4426$ & COU1652 & 2012.868 & 1 & 0.658 & 0.008 & $99.8^{*}$ & 0.8 & & & & & \\
\hline $00402+4117$ & TDS1562 & 2012.920 & 1 & 1.669 & 0.008 & $307.8^{*}$ & * 0.3 & & & & & \\
\hline $00407+3707$ & COU852 & 2011.585 & 1 & 0.906 & 0.010 & $250.6^{*}$ & * 0.3 & & & & & \\
\hline $00408+1308$ & HEI302 & 2011.879 & 1 & - & - & - & - & & NR & & & \\
\hline $00408+1308$ & HEI302 & 2012.999 & 1 & 0.618 & 0.007 & $89.3^{*}$ & 0.5 & & & & & \\
\hline $00408+3844$ & COU853 & 2011.585 & 1 & 0.888 & 0.007 & $89.7^{*}$ & 0.3 & & & & & \\
\hline $00416+1452$ & HEI92 & 2011.879 & 1 & 1.179 & 0.007 & $109.9^{*}$ & * 0.3 & 3.60 & & & & \\
\hline $00419+1751$ & A 2303 & 2012.879 & 1 & 1.097 & 0.007 & $218.9^{*}$ & * 0.3 & & & & & \\
\hline $00423+0449$ & HDS92 & 2012.873 & 1 & 0.190 & 0.040 & 167.1 & 8.0 & & & & & \\
\hline $00425+4808$ & COU1854 & 2012.920 & 1 & 1.058 & 0.007 & 86.7 & 0.3 & & & & & \\
\hline $00428+1249$ & HDS93 & 2012.873 & 1 & 0.284 & 0.015 & $33.6^{*}$ & 3.0 & & & Cve2017b & -0.01 & -5.5 \\
\hline $00429+2047$ & A 2205 & 2012.879 & 1 & 0.351 & 0.007 & $188.0^{*}$ & * 0.3 & & & Msn2017a & -0.01 & $2.6^{Q}$ \\
\hline $00430-0351$ & HDS95 & 2015.413 & 1 & - & - & - & - & & NR & & & \\
\hline $00431+7659$ & HU1012 & 2012.988 & 1 & 0.432 & 0.008 & $121.4^{*}$ & * 0.3 & & & USN2002 & -0.07 & 2.3 \\
\hline $00433+0634$ & HDS96 & 2012.873 & 1 & 0.242 & 0.015 & $324.2^{*}$ & * 0.4 & & & & & \\
\hline $00443+0554$ & HDS97 & 2012.873 & 1 & 1.427 & 0.024 & 141.2 & 1.4 & & & & & \\
\hline $00448+7700$ & HU1014 & 2012.988 & 1 & 0.723 & 0.007 & $162.1^{*}$ & * 0.6 & & & & & \\
\hline $00457+0654$ & A2002 & 2012.879 & 1 & 0.177 & 0.040 & 114.0 & 8.0 & & & & & \\
\hline $00458+5108$ & BU493 & 2012.920 & 1 & 0.860 & 0.007 & $54.0^{*}$ & 0.3 & & & & & \\
\hline $00463+4715$ & COU2002 & 2012.920 & 1 & 0.168 & 0.040 & 92.0 & 8.0 & & & & & \\
\hline $00465+1558$ & HEI19 & 2012.879 & 1 & 0.618 & 0.007 & $245.7^{*}$ & * 0.5 & & & & & \\
\hline $00470+2315$ & HU413 & 2012.879 & 1 & 0.325 & 0.008 & $321.5^{*}$ & * 1.1 & & & Ole2003d & -0.05 & 0.8 \\
\hline $00477+4017$ & COU1206 & 2011.585 & 1 & 0.370 & 0.015 & $260.8^{*}$ & ${ }^{*} 0.7$ & & & & & \\
\hline $00484+4701$ & COU2003 & 2012.868 & 1 & 0.711 & 0.007 & $207.1^{*}$ & * 0.3 & & & & & \\
\hline
\end{tabular}




\begin{tabular}{|c|c|c|c|c|c|c|c|c|c|c|c|c|}
\hline WDS & Name & Epoch & Bin. & $\begin{array}{c}\rho \\
(")\end{array}$ & $\begin{array}{l}\sigma_{\rho} \\
(")\end{array}$ & $\begin{array}{c}\theta \\
\left(^{\circ}\right)\end{array}$ & $\begin{array}{l}\sigma_{\theta} \\
\left({ }^{\circ}\right)\end{array}$ & $\Delta m$ & Notes & Orbit & $\begin{array}{r}\Delta \rho(\mathrm{O}-\mathrm{C}) \\
(")\end{array}$ & $\begin{array}{r}\Delta \theta(\mathrm{O}-\mathrm{C}) \\
\left({ }^{\circ}\right)\end{array}$ \\
\hline $00487+1841$ & BU495AB & 2011.582 & 1 & 0.300 & 0.028 & $250.3^{*}$ & * 0.7 & & & Sca2000b & -0.03 & 2.2 \\
\hline $00487+1841$ & BU495AB & 2011.582 & 1 & 0.291 & 0.026 & $249.4^{*}$ & $* 2.1$ & & & Sca2000b & -0.04 & 1.3 \\
\hline $00487+3604$ & COU1207 & 2011.585 & 1 & 1.758 & 0.009 & $323.6^{*}$ & * 0.3 & & & & & \\
\hline $00488+1116$ & TDS1627 & 2011.879 & 1 & - & - & - & - & & NR & & & \\
\hline $00494+1104$ & HDS108 & 2011.879 & 1 & 0.243 & 0.007 & $162.9^{*}$ & $* 2.3$ & & & & & \\
\hline $00495+4018$ & HDS110 & 2012.920 & 1 & 0.544 & 0.027 & 16.2 & 6.3 & 2.22 & & & & \\
\hline $00495+4018$ & HDS110 & 2012.920 & 1 & 0.565 & 0.037 & 9.6 & 7.1 & & & & & \\
\hline $00496+4128$ & COU1503 & 2012.920 & 1 & 1.015 & 0.007 & $296.1^{*}$ & * 0.5 & & & & & \\
\hline $00508+3203$ & $\mathrm{~A} 922 \mathrm{Aa}, \mathrm{Ab}$ & 2011.582 & 1 & 0.567 & 0.007 & $154.3^{*}$ & * 0.3 & & & Msn2001c & 0.07 & $-5.6^{Q}$ \\
\hline $00512+1405$ & YSC18 & 2012.879 & 1 & 0.135 & 0.040 & 48.6 & 8.0 & & & & & \\
\hline $00516+2237$ & A1808 & 2012.980 & 1 & 0.197 & 0.040 & $194.5^{*}$ & $* 8.0$ & & & Doc2005b & -0.00 & -8.2 \\
\hline $00516+2237$ & A1808 & 2011.582 & 1 & 0.151 & 0.069 & $197.9^{*}$ & $* 8.0$ & & & Doc2005b & -0.05 & -3.9 \\
\hline $00516+6859$ & BU781 & 2012.988 & 1 & 1.065 & 0.007 & $23.0^{*}$ & 0.3 & & & & & \\
\hline $00520+3154$ & A924 & 2011.582 & 1 & - & - & - & - & & NR & & & \\
\hline $00521+4326$ & COU1653 & 2012.868 & 1 & 0.199 & 0.040 & $21.1^{*}$ & 8.0 & & & & & \\
\hline $00525+1740$ & A2306 & 2012.980 & 1 & 1.390 & 0.007 & $302.1^{*}$ & * 0.3 & & & & & \\
\hline $00533+0405$ & A2307 & 2012.980 & 1 & 0.131 & 0.040 & 217.5 & 8.0 & & & Tok2015c & -0.00 & -6.5 \\
\hline $00542+4318$ & COU1654 & 2012.868 & 1 & - & - & - & - & & NR & & & \\
\hline $00544+4148$ & COU1355 & 2012.868 & 1 & 0.452 & 0.007 & $121.9^{*}$ & * 0.4 & & & & & \\
\hline $00546+1911$ & STT20AB & 2011.582 & 1 & 0.584 & 0.007 & $182.4^{*}$ & * 0.3 & & & Doc2014a & 0.01 & 1.3 \\
\hline $00549+1928$ & A2208 & 2012.980 & 1 & 1.559 & 0.008 & $90.9^{*}$ & 0.3 & & & & & \\
\hline $00567+1010$ & HDS126 & 2012.999 & 1 & 0.796 & 0.010 & $65.1^{*}$ & 0.4 & & & & & \\
\hline $00571+1239$ & HEI304 & 2011.879 & 1 & 0.760 & 0.007 & $267.6^{*}$ & * 0.3 & 0.20 & & & & \\
\hline $00575+3738$ & COU1054 & 2011.585 & 1 & 0.178 & 0.10 & 149.7 & 5.5 & & & & & \\
\hline $00581+4751$ & COU2253 & 2012.868 & 1 & 1.043 & 0.010 & $13.6^{*}$ & 0.4 & & & & & \\
\hline $00583+2124$ & BU302 & 2012.999 & 1 & 0.246 & 0.007 & 209.7 & 0.3 & & & Cve2006e & 0.04 & -17.4 \\
\hline $00583+2124$ & BU302 & 2012.999 & 1 & 0.255 & 0.034 & $212.0^{*}$ & * 3.8 & & & Cve2006e & 0.05 & -15.1 \\
\hline $00585+4843$ & COU2254 & 2012.920 & 1 & 0.466 & 0.007 & $161.9^{*}$ & $* 0.6$ & & & & & \\
\hline $00599+4257$ & COU1655 & 2012.868 & 1 & 1.250 & 0.007 & $2.8^{*}$ & 0.3 & & & & & \\
\hline $01005+1841$ & HDS132 & 2012.999 & 1 & 0.384 & 0.007 & $137.6^{*}$ & * 0.3 & & & & & \\
\hline $01007+0929$ & STF82AB & 2011.991 & 1 & 1.807 & 0.009 & $304.8^{*}$ & * 0.3 & 5.40 & & & & \\
\hline $01007+1659$ & HEI96 & 2012.879 & 1 & 0.399 & 0.007 & $66.5^{*}$ & 0.7 & & & & & \\
\hline $01007+4344$ & COU1656 & 2012.920 & 1 & 1.556 & 0.008 & $25.5^{*}$ & 0.5 & & & & & \\
\hline $01010+4830$ & COU2256 & 2012.868 & 1 & 0.585 & 0.007 & $127.2^{*}$ & * 0.3 & & & & & \\
\hline $01014+1155$ & BU867 & 2012.980 & 1 & 0.669 & 0.007 & $354.6^{*}$ & $* 0.5$ & & & Hrt2008 & 0.04 & 0.9 \\
\hline $01014+3535$ & COU854 & 2011.585 & 1 & 0.176 & 0.040 & 16.4 & 8.0 & & & Hrt2009 & 0.04 & -3.7 \\
\hline $01021+3847$ & COU1210 & 2012.920 & 1 & 0.412 & 0.007 & $81.4^{*}$ & 0.5 & & & & & \\
\hline $01023+0552$ & A2003 & 2012.879 & 1 & - & - & - & - & & NR & & & \\
\hline $01024+0504$ & HDS135 & 2012.999 & 1 & 0.717 & 0.007 & $80.8^{*}$ & 0.7 & & & Tok2015c & -0.01 & -0.6 \\
\hline $01026+4900$ & COU1855 & 2012.868 & 1 & 0.936 & 0.007 & $104.2^{*}$ & $* 0.3$ & & & & & \\
\hline $01030+7934$ & MLR372 & 2012.988 & 1 & - & - & - & - & & NR & & & \\
\hline $01039+4004$ & COU1055 & 2011.585 & 1 & 1.374 & 0.007 & $297.1^{*}$ & * 0.3 & & & & & \\
\hline $01049+3649$ & A1515 & 2011.582 & 1 & 0.191 & 0.040 & 310.0 & 8.0 & & & Doc2009g & 0.01 & -0.3 \\
\hline $01050+5029$ & COU1856 & 2012.868 & 1 & 0.118 & 0.040 & 100.1 & 8.0 & & & & & \\
\hline $01054+4340$ & COU1657 & 2012.920 & 1 & 0.277 & 0.007 & 81.0 & 0.3 & & & & & \\
\hline $01055+2107$ & AG14 & 2012.980 & 1 & 0.801 & 0.037 & $312.5^{*}$ & * 2.3 & & & FMR2014f & 0.01 & -0.8 \\
\hline $01055+2107$ & AG14 & 2011.585 & 1 & 0.765 & 0.037 & $314.5^{*}$ & $* 2.3$ & & & FMR2014f & -0.01 & -0.0 \\
\hline $01064+8005$ & MLR445 & 2012.988 & 1 & 0.258 & 0.007 & $163.6^{*}$ & $* 3.2$ & & & & & \\
\hline $01065+2834$ & VKI2 & 2011.585 & 1 & 2.013 & 0.010 & $185.4^{*}$ & $* 0.4$ & & & & & \\
\hline $01072+3839$ & $\mathrm{~A} 1516 \mathrm{AB}$ & 2011.582 & 1 & 0.154 & 0.040 & 5.8 & 8.0 & & & Doc2017i & 0.01 & -1.8 \\
\hline $01072+3839$ & A1516AB & 2011.582 & 1 & - & - & - & - & & NR & & & \\
\hline $01080+1204$ & A2101 & 2012.999 & 1 & 0.618 & 0.007 & $79.6^{*}$ & 0.3 & & & & & \\
\hline $01080+2044$ & DRS2 & 2015.417 & 1 & - & - & - & - & & NR & & & \\
\hline
\end{tabular}




\begin{tabular}{|c|c|c|c|c|c|c|c|c|c|c|c|c|}
\hline WDS & Name & Epoch & Bin. & $\begin{array}{c}\rho \\
(")\end{array}$ & $\begin{array}{l}\sigma_{\rho} \\
(")\end{array}$ & $\begin{array}{c}\theta \\
\left(^{\circ}\right)\end{array}$ & $\begin{array}{l}\sigma_{\theta} \\
\left(^{\circ}\right)\end{array}$ & $\Delta m$ & Notes & Orbit & $\begin{array}{r}\Delta \rho(\mathrm{O}-\mathrm{C}) \\
\quad(")\end{array}$ & $\begin{array}{r}\Delta \theta(\mathrm{O}-\mathrm{C}) \\
\left({ }^{\circ}\right)\end{array}$ \\
\hline $01088+3024$ & HDS149 & 2011.879 & 1 & 0.441 & 0.030 & $188.8^{*}$ & * 2.1 & & & & & \\
\hline $01088+3441$ & COU662 & 2011.585 & 1 & 1.059 & 0.007 & $251.0^{*}$ & ${ }^{*} 0.3$ & & & & & \\
\hline $01105+3917$ & COU1212 & 2012.585 & 1 & - & - & - & - & & NR & & & \\
\hline $01105+3917$ & COU1212 & 2012.868 & 1 & - & - & - & - & & NR & & & \\
\hline $01108+6747$ & HDS155 & 2012.988 & 1 & 0.154 & 0.040 & $165.3^{*}$ & * 8.0 & & & Doc2018i & 0.03 & -0.7 \\
\hline $01112+3743$ & HO215 & 2011.585 & 1 & - & - & - & - & & NR & & & \\
\hline $01117+0835$ & HDS158 & 2012.879 & 1 & 0.257 & 0.011 & $138.6^{*}$ & * 1.3 & & & & & \\
\hline $01131+2942$ & A1260 & 2011.585 & 1 & 0.272 & 0.034 & $227.4^{*}$ & * 5.6 & & & Msn2017a & 0.03 & $-12.8^{Q}$ \\
\hline $01131+2942$ & A 1260 & 2011.879 & 1 & 0.214 & 0.020 & $244.0^{*}$ & * 4.2 & & & Msn2017a & -0.03 & $3.6^{Q}$ \\
\hline $01131+2942$ & A1260 & 2011.991 & 1 & 0.201 & 0.020 & $231.4^{*}$ & * 3.2 & & & Msn2017a & -0.04 & $-9.1^{Q}$ \\
\hline $01140+2015$ & TDS1809 & 2012.980 & 1 & - & - & - & - & & NR & & & \\
\hline $01154+3829$ & HO6 & 2011.991 & 1 & 1.273 & 0.007 & $122.8^{*}$ & * 0.3 & & & & & \\
\hline $01158+0947$ & A2102 & 2011.582 & 1 & 0.283 & 0.037 & $116.5^{*}$ & * 1.9 & & & RAO2015 & -0.00 & 2.0 \\
\hline $01158+0947$ & A2102 & 2012.879 & 1 & 0.204 & 0.035 & $112.5^{*}$ & * 1.0 & & & RAO2015 & -0.07 & 0.5 \\
\hline $01162+7145$ & HDS167 & 2012.988 & 1 & - & - & - & - & & NR & & & \\
\hline $01184+6333$ & HDS171 & 2012.988 & 1 & 0.937 & 0.007 & $88.6^{*}$ & 1.5 & & & & & \\
\hline $01185+7323$ & A814 & 2012.988 & 1 & 0.220 & 0.007 & $193.5^{*}$ & * 0.3 & & & & & \\
\hline $01189+3347$ & COU664 & 2011.879 & 1 & 2.095 & 0.010 & $324.2^{*}$ & * 0.3 & 1.30 & & & & \\
\hline $01197+1209$ & CHR196 & 2012.999 & 1 & 0.154 & 0.046 & 136.2 & 8.0 & & & Tok2018i & 0.06 & 14.1 \\
\hline $01213+1132$ & BU4AB & 2011.582 & 1 & 0.590 & 0.027 & $108.0^{*}$ & * 2.3 & & & Sca2015b & 0.01 & -0.2 \\
\hline $01213+1132$ & BU4AB & 2011.991 & 1 & 0.605 & 0.027 & $106.5^{*}$ & * 2.4 & & & Sca2015b & 0.03 & -1.6 \\
\hline $01213+1132$ & BU4AB & 2012.980 & 1 & 0.580 & 0.027 & $107.4^{*}$ & * 2.3 & & & Sca2015b & -0.00 & -0.5 \\
\hline $01221+0939$ & HEI 428 & 2012.879 & 1 & 1.204 & 0.007 & $339.8^{*}$ & ${ }^{*} 0.3$ & & & & & \\
\hline $01226+1449$ & MRL1 & 2011.879 & 1 & 0.239 & 0.038 & $125.8^{*}$ & * 3.5 & 0.10 & & & & \\
\hline $01226+1449$ & MRL1 & 2012.879 & 1 & 0.252 & 0.037 & $117.1^{*}$ & * 3.3 & & & & & \\
\hline $01230+4005$ & COU1213 & 2012.920 & 1 & 1.468 & 0.007 & $99.4^{*}$ & 0.3 & & & & & \\
\hline $01238+0630$ & HDS180 & 2012.879 & 1 & 0.183 & 0.040 & $174.5^{*}$ & * 8.0 & & & & & \\
\hline $01244+3653$ & HDS181 & 2011.991 & 1 & 0.230 & 0.009 & 174.8 & 1.1 & & & & & \\
\hline $01246+4310$ & A3001 & 2012.920 & 1 & 0.324 & 0.067 & $89.9^{*}$ & 4.3 & & & & & \\
\hline $01251+4537$ & A939 & 2012.920 & 1 & 0.175 & 0.040 & $189.6^{*}$ & * 8.0 & & & Msn2014b & -0.03 & -6.5 \\
\hline $01255+7407$ & HDS185 & 2012.988 & 1 & 0.209 & 0.007 & $227.8^{*}$ & * 1.4 & & & & & \\
\hline $01256+2331$ & TOK11 & 2011.879 & 1 & - & - & - & - & & NR & & & \\
\hline $01260+1315$ & HEI305 & 2012.879 & 1 & 0.461 & 0.007 & $143.8^{*}$ & * 0.3 & & & & & \\
\hline $01264+1451$ & HEI205 & 2012.980 & 1 & 0.405 & 0.007 & $64.3^{*}$ & 0.6 & & & & & \\
\hline $01276+2104$ & COU148 & 2011.991 & 1 & 1.223 & 0.007 & $247.2^{*}$ & * 0.3 & 0.80 & & & & \\
\hline $01277+0521$ & BU1164AB & 2011.582 & 1 & 0.179 & 0.040 & $172.7^{*}$ & * 8.0 & & & Doc2010i & 0.07 & -12.9 \\
\hline $01277+0521$ & BU1164AB & 2011.582 & 1 & 0.162 & 0.040 & $176.5^{*}$ & * 8.0 & & & Doc2010i & 0.05 & -9.1 \\
\hline $01277+0521$ & BU1164AB & 2012.999 & 1 & 0.156 & 0.040 & 169.1 & 8.0 & & & Doc2010i & -0.01 & -9.1 \\
\hline $01284+0758$ & YR7 & 2012.980 & 1 & 0.508 & 0.007 & $40.9^{*}$ & 0.3 & & & & & \\
\hline $01286+4509$ & A941CD & 2012.920 & 1 & 0.770 & 0.007 & $358.3^{*}$ & * 0.6 & & & & & \\
\hline $01292+2004$ & A2214 & 2011.991 & 1 & 0.729 & 0.007 & $212.0^{*}$ & * 0.9 & & & & & \\
\hline $01292+4321$ & COU1359 & 2012.920 & 1 & 0.943 & 0.007 & $140.0^{*}$ & ${ }^{*} 0.3$ & & & & & \\
\hline $01297+2250$ & A1910 & 2011.585 & 1 & 0.200 & 0.007 & $182.4^{*}$ & * 0.6 & & & Hrt1996a & 0.01 & -0.4 \\
\hline $01298+1014$ & CHR197 & 2012.980 & 1 & 0.232 & 0.007 & 13.3 & 0.7 & & & & & \\
\hline $01298+6908$ & MLR295 & 2012.988 & 1 & 0.308 & 0.007 & $177.8^{*}$ & * 1.1 & & & & & \\
\hline $01308+2311$ & HDS197 & 2012.999 & 1 & 0.125 & 0.040 & 161.5 & 8.0 & & & & & \\
\hline $01317+1506$ & CHR198 & 2012.980 & 1 & 0.446 & 0.027 & $358.8^{*}$ & * 1.3 & 1.66 & & & & \\
\hline $01317+1506$ & CHR198 & 2012.980 & 1 & 0.404 & 0.037 & 357.6 & 1.6 & & & & & \\
\hline $01328+3551$ & STF133AB & 2011.030 & 1 & 3.060 & 0.015 & $192.0^{*}$ & * 0.3 & & & & & \\
\hline $01328+3551$ & A1911 & 2011.991 & 1 & 0.286 & 0.007 & $358.9^{*}$ & * 1.5 & & & & & \\
\hline $01334+7438$ & HDS204 & 2012.988 & 1 & 0.255 & 0.008 & 37.7 & 1.2 & & & & & \\
\hline $01345+3440$ & A1913 & 2011.879 & 1 & 0.405 & 0.010 & $311.1^{*}$ & * 2.0 & 0.00 & & Mnt1999a & 0.08 & $3.8^{Q}$ \\
\hline $01345+7804$ & $\mathrm{HDS} 211 \mathrm{Aa}, \mathrm{Ab}$ & 2012.988 & 1 & - & - & - & - & & NR & & & \\
\hline
\end{tabular}




\begin{tabular}{|c|c|c|c|c|c|c|c|c|c|c|c|c|}
\hline WDS & Name & Epoch & Bin. & $\begin{array}{c}\rho \\
(")\end{array}$ & $\begin{array}{l}\sigma_{\rho} \\
(")\end{array}$ & $\begin{array}{c}\theta \\
\left({ }^{\circ}\right)\end{array}$ & $\begin{array}{l}\sigma_{\theta} \\
\left({ }^{\circ}\right)\end{array}$ & $\Delta m$ & Notes & Orbit & $\begin{array}{r}\Delta \rho(\mathrm{O}-\mathrm{C}) \\
(")\end{array}$ & $\begin{array}{r}\Delta \theta(\mathrm{O}-\mathrm{C}) \\
\left({ }^{\circ}\right)\end{array}$ \\
\hline $01354+1126$ & HDS213 & 2012.999 & 1 & 0.176 & 0.269 & $198.8^{*}$ & 11.3 & & & Cve2017b & -0.07 & 10.9 \\
\hline $01356+7227$ & A816 & 2012.988 & 1 & 0.906 & 0.007 & $129.1^{*}$ & 0.3 & & & & & \\
\hline $01364+1311$ & HEI306Aa,Ab & 2011.880 & 1 & - & - & - & - & & NR & & & \\
\hline $01377+1836$ & COU254 & 2011.991 & 1 & 1.675 & 0.008 & $23.8^{*}$ & 0.3 & 0.20 & & & & \\
\hline $01384+3828$ & COU1215 & 2011.991 & 1 & 0.494 & 0.007 & $154.3^{*}$ & 0.4 & 0.20 & & & & \\
\hline $01385+3448$ & COU1059 & 2011.880 & 1 & 0.329 & 0.037 & $128.9^{*}$ & 4.5 & & & & & \\
\hline $01385+3448$ & COU1059 & 2011.991 & 1 & 0.351 & 0.037 & $133.3^{*}$ & 4.5 & 0.40 & & & & \\
\hline $01388+3453$ & COU1060 & 2011.991 & 1 & 0.536 & 0.007 & $191.7^{*}$ & 0.8 & 1.00 & & & & \\
\hline $01390+0407$ & A2403 & 2012.980 & 1 & 0.673 & 0.007 & $93.2^{*}$ & 1.1 & & & & & \\
\hline $01395+3310$ & COU749 & 2012.920 & 1 & 2.295 & 0.011 & $16.9^{*}$ & 0.3 & & & & & \\
\hline $01409+1117$ & A 2320 & 2012.980 & 1 & 0.226 & 0.007 & $103.8^{*}$ & 1.0 & & & Doc2007a & 0.02 & $0.8^{Q}$ \\
\hline $01425+6606$ & HDS225 & 2012.988 & 1 & 0.234 & 0.012 & $259.5^{*}$ & 5.2 & & & & & \\
\hline $01432+0520$ & A2008 & 2012.980 & 1 & 0.281 & 0.007 & $176.3^{*}$ & 1.7 & & & & & \\
\hline $01432+3130$ & COU449 & 2011.880 & 1 & 0.196 & 0.040 & $144.0^{*}$ & 8.0 & & & & & \\
\hline $01435+1927$ & COU256 & 2012.999 & 1 & 0.165 & 0.040 & 92.8 & 8.0 & & & & & \\
\hline $01436+7741$ & HDS231 & 2012.988 & 1 & - & - & - & - & & NR & & & \\
\hline $01437+0934$ & BU509 & 2012.980 & 1 & 0.723 & 0.007 & $45.3^{*}$ & 0.3 & & & Hrt2010a & -0.03 & -1.5 \\
\hline $01437+3950$ & COU1217 & 2011.991 & 1 & 0.537 & 0.007 & $333.4^{*}$ & 0.4 & 0.40 & & & & \\
\hline $01445+0749$ & HEI430 & 2012.980 & 1 & 1.032 & 0.007 & $74.3^{*}$ & 0.3 & & & & & \\
\hline $01447+1906$ & COU257 & 2011.880 & 1 & 1.505 & 0.008 & $335.3^{*}$ & 0.3 & & & & & \\
\hline $01449+1951$ & A 2322 & 2012.999 & 1 & 0.197 & 0.040 & $195.9^{*}$ & 8.0 & & & & & \\
\hline $01450+2703$ & COU750 & 2011.880 & 1 & 0.297 & 0.007 & $28.0^{*}$ & 1.2 & & & Doc2005c & -0.06 & -2.4 \\
\hline $01459+0411$ & A2405 & 2012.980 & 1 & 0.923 & 0.007 & $221.6^{*}$ & 0.3 & & & & & \\
\hline $01461+6349$ & STF148 & 2012.988 & 1 & 0.168 & 0.085 & $58.8^{*}$ & 16.3 & & & Nov2007d & -0.04 & $-56.5^{Q}$ \\
\hline $01474+5113$ & COU2676 & 2011.521 & 1 & 1.130 & 0.007 & 338.3 & 0.3 & 1.56 & & & & \\
\hline $01499+8053$ & STT34 & 2012.988 & 1 & 0.511 & 0.007 & $109.5^{*}$ & 0.5 & & & Hei1997 & 0.08 & $-2.9^{Q}$ \\
\hline $01509+4037$ & COU1361 & 2012.920 & 1 & 1.162 & 0.007 & $280.7^{*}$ & 0.3 & & & & & \\
\hline $01510+2551$ & COU452 & 2011.880 & 1 & 0.204 & 0.015 & $177.2^{*}$ & 1.7 & & & & & \\
\hline $01518+1817$ & COU2557 & 2011.992 & 1 & 1.212 & 0.007 & 79.6 & 0.3 & & & & & \\
\hline $01520+1326$ & HU1213 & 2012.980 & 1 & - & - & - & - & & NR & & & \\
\hline $01523+3711$ & COU1066 & 2012.920 & 1 & 0.902 & 0.007 & $145.3^{*}$ & 0.9 & & & & & \\
\hline $01544+1754$ & COU352 & 2012.999 & 1 & 0.960 & 0.037 & 21.8 & 0.5 & & & & & \\
\hline $01544+1754$ & COU352 & 2011.880 & 1 & 1.001 & 0.037 & $21.2^{*}$ & 0.3 & & & & & \\
\hline $01546+1405$ & HEI208 & 2012.980 & 1 & 1.391 & 0.007 & 180.2 & 0.5 & & & & & \\
\hline $01547+4426$ & COU1508 & 2012.920 & 1 & 0.313 & 0.007 & $49.9^{*}$ & 0.5 & & & & & \\
\hline $01563+2520$ & COU453 & 2011.030 & 1 & 0.687 & 0.007 & $280.3^{*}$ & 0.5 & & & & & \\
\hline $01572+7143$ & HDS264 & 2012.988 & 1 & 0.565 & 0.010 & 92.5 & 0.5 & & & & & \\
\hline $01581+3444$ & COU859 & 2011.880 & 1 & 0.242 & 0.007 & 2.8 & 0.3 & & & & & \\
\hline $01585+1048$ & YSC85 & 2012.980 & 1 & 0.274 & 0.010 & $174.1^{*}$ & 0.9 & & & & & \\
\hline $01585+1048$ & YSC85 & 2011.992 & 2 & 0.336 & 0.040 & $168.9^{*}$ & 8.0 & & & & & \\
\hline $01588+3730$ & COU1364 & 2012.920 & 1 & 0.480 & 0.007 & $53.4^{*}$ & 0.9 & & & & & \\
\hline $01596+3044$ & COU669 & 2011.992 & 1 & 0.994 & 0.007 & $289.4^{*}$ & 0.5 & 0.20 & & & & \\
\hline $02005+3958$ & HDS268 & 2011.992 & 1 & 0.927 & 0.007 & 18.4 & 0.3 & & & & & \\
\hline $02012+1634$ & BU515 & 2011.880 & 1 & 0.390 & 0.037 & 102.7 & 1.1 & & & & & \\
\hline $02012+1634$ & BU515 & 2011.880 & 1 & - & - & - & - & & NR & & & \\
\hline $02012+1634$ & BU515 & 2011.992 & 1 & 0.379 & 0.030 & 99.5 & 2.0 & & & & & \\
\hline $02019+4831$ & COU2009 & 2012.920 & 1 & 0.580 & 0.007 & $59.0^{*}$ & 0.6 & & & & & \\
\hline $02021+5318$ & A3106 & 2012.038 & 1 & - & - & - & - & & NR & & & \\
\hline $02026+0905$ & MCA4 & 2012.999 & 1 & 0.176 & 0.040 & 154.9 & 8.0 & & & & & \\
\hline $02029+1938$ & COU2677 & 2011.992 & 1 & 1.576 & 0.008 & $90.4^{*}$ & 0.3 & & & & & \\
\hline $02055+1029$ & HEI436 & 2012.999 & 1 & 2.078 & 0.050 & $55.5^{*}$ & 0.9 & & & & & \\
\hline $02062+2507$ & STF212 & 2011.030 & 1 & 1.926 & 0.010 & $162.3^{*}$ & 0.3 & & & & & \\
\hline
\end{tabular}




\begin{tabular}{|c|c|c|c|c|c|c|c|c|c|c|c|c|}
\hline WDS & Name & Epoch & Bin. & $\begin{array}{c}\rho \\
(")\end{array}$ & $\begin{array}{l}\sigma_{\rho} \\
(")\end{array}$ & $\begin{array}{c}\theta \\
\left(^{\circ}\right)\end{array}$ & $\begin{array}{l}\sigma_{\theta} \\
\left(^{\circ}\right)\end{array}$ & $\Delta m$ & Notes & Orbit & $\begin{array}{r}\Delta \rho(\mathrm{O}-\mathrm{C}) \\
(")\end{array}$ & $\begin{array}{r}\Delta \theta(\mathrm{O}-\mathrm{C}) \\
\left({ }^{\circ}\right)\end{array}$ \\
\hline $02071+6957$ & STF204 & 2012.988 & 1 & 0.187 & 0.040 & $34.6^{*}$ & 8.0 & & & & & \\
\hline $02085+4959$ & COU2562 & 2012.920 & 1 & 1.139 & 0.007 & $175.3^{*}$ & $* 0.4$ & & & & & \\
\hline $02085+5852$ & HDS284 & 2012.038 & 1 & 0.231 & 0.007 & $76.3^{*}$ & 1.0 & & & & & \\
\hline $02087+1045$ & HEI437 & 2012.999 & 1 & - & - & - & - & & $\mathrm{NR}$ & & & \\
\hline $02089+7032$ & MLR376 & 2012.988 & 1 & 0.334 & 0.007 & $30.0^{*}$ & 0.5 & & & & & \\
\hline $02090+3540$ & COU1067 & 2011.992 & 1 & 0.176 & 0.040 & 35.5 & 8.0 & & & & & \\
\hline $02096+1000$ & TDS2139 & 2012.999 & 1 & - & - & - & - & & $\mathrm{NR}$ & & & \\
\hline $02105+8129$ & STT37 & 2012.988 & 1 & 1.175 & 0.007 & $208.7^{*}$ & * 0.4 & & & & & \\
\hline $02126+1548$ & HU1215 & 2012.999 & 1 & 1.573 & 0.010 & $334.7^{*}$ & * 0.3 & & & & & \\
\hline $02140+2342$ & HU424 & 2012.999 & 1 & 1.350 & 0.007 & $335.9^{*}$ & * 0.3 & & & & & \\
\hline $02140+3431$ & STF229 & 2011.030 & 1 & 2.476 & 0.012 & $356.0^{*}$ & * 0.3 & & & & & \\
\hline $02142+0909$ & HDS297 & 2012.999 & 1 & - & - & - & - & & $\mathrm{NR}$ & & & \\
\hline $02144+3810$ & ES230 & 2011.030 & 1 & 2.768 & 0.024 & $302.1^{*}$ & * 0.3 & & & & & \\
\hline $02150+5903$ & MLR447 & 2012.038 & 1 & 0.666 & 0.007 & $341.7^{*}$ & * 0.3 & 1.70 & & & & \\
\hline $02156+5932$ & MLR448 & 2012.038 & 1 & 0.162 & 0.040 & 134.7 & 8.0 & & & & & \\
\hline $02157+2503$ & COU79 & 2011.992 & 1 & 0.125 & 0.040 & 42.0 & 8.0 & & & Mut2010e & -0.04 & -8.5 \\
\hline $02162+0919$ & HEI438 & 2012.999 & 1 & 0.696 & 0.007 & $163.1^{*}$ & $* 0.7$ & & & & & \\
\hline $02180+1251$ & HEI310 & 2012.999 & 1 & 1.238 & 0.010 & $177.6^{*}$ & * 0.7 & & & & & \\
\hline $02181+5731$ & BU1170BC & 2012.038 & 1 & 0.471 & 0.010 & $129.4^{*}$ & * 1.3 & & & & & \\
\hline $02211+8044$ & STF223 & 2012.988 & 1 & 0.806 & 0.007 & $47.8^{*}$ & 0.4 & & & & & \\
\hline $02212+2136$ & HU425 & 2011.992 & 1 & 0.616 & 0.008 & $146.4^{*}$ & * 0.6 & 0.90 & & & & \\
\hline $02212+2751$ & COU457 & 2011.992 & 1 & 0.484 & 0.008 & $161.1^{*}$ & * 0.4 & 1.10 & & & & \\
\hline $02217+7623$ & STF233 & 2012.988 & 1 & 1.961 & 0.010 & $272.4^{*}$ & * 0.4 & & & & & \\
\hline $02225+0948$ & HEI439 & 2012.999 & 1 & 1.427 & 0.007 & 32.6 & 0.3 & & & & & \\
\hline $02231+7021$ & MLR377 & 2012.988 & 1 & 0.677 & 0.008 & $140.3^{*}$ & * 0.5 & & & Msn2017e & -0.02 & 2.5 \\
\hline $02277+7147$ & MLR305 & 2012.988 & 1 & 0.210 & 0.013 & 79.6 & 3.6 & & & & & \\
\hline $02277+7147$ & HJ1116AB & 2012.988 & 1 & 4.362 & 0.023 & $131.8^{*}$ & * 0.3 & & & & & \\
\hline $02278+0426$ & A2329 & 2012.999 & 1 & 0.297 & 0.007 & $305.0^{*}$ & * 0.3 & & & Ana2007 & -0.03 & 1.9 \\
\hline $02282+2320$ & HU428 & 2012.999 & 1 & - & - & - & - & & NR & & & \\
\hline $02284+1722$ & A2330 & 2012.999 & 1 & 1.210 & 0.007 & $209.8^{*}$ & * 0.4 & & & Izm2019 & 0.01 & 2.3 \\
\hline $02287+0840$ & A2016 & 2012.999 & 1 & 0.416 & 0.007 & $179.7^{*}$ & * 0.3 & & & & & \\
\hline $02291+2331$ & VBS6 & 2012.999 & 1 & 0.458 & 0.007 & $48.8^{*}$ & 1.3 & & & & & \\
\hline $02303+7025$ & HDS327 & 2012.988 & 1 & - & - & - & - & & $\mathrm{NR}$ & & & \\
\hline $02303+7025$ & HDS327 & 2012.030 & 1 & 1.565 & 0.014 & $179.8^{*}$ & ${ }^{*} 0.4$ & & & & & \\
\hline $02361+7944$ & MLR449 & 2012.038 & 1 & 0.294 & 0.007 & $202.1^{*}$ & * 0.5 & & & & & \\
\hline $02379+2003$ & A2219 & 2011.066 & 1 & 0.480 & 0.009 & $146.4^{*}$ & * 0.3 & & & Msn2017a & 0.04 & -0.9 \\
\hline $02392+1822$ & COU2679 & 2012.999 & 1 & 1.367 & 0.007 & $118.7^{*}$ & * 0.3 & & & & & \\
\hline $02396+6229$ & MLR32 & 2011.521 & 1 & 1.871 & 0.009 & 147.3 & 0.3 & & & & & \\
\hline $02402+0436$ & HDS347 & 2012.999 & 1 & 0.515 & 0.015 & $317.6^{*}$ & * 1.8 & & & Cve2020b & -0.04 & $-0.2^{Q}$ \\
\hline $02404+5248$ & HDS348 & 2012.038 & 1 & 0.903 & 0.007 & $80.9^{*}$ & 0.4 & & & & & \\
\hline $02405+1801$ & COU462 & 2011.030 & 1 & - & - & - & - & & NR & & & \\
\hline $02409+0452$ & STT45 & 2011.030 & 1 & 0.795 & 0.007 & $263.8^{*}$ & * 0.3 & & & Izm2019 & -0.00 & 2.6 \\
\hline $02410+5222$ & MLR657 & 2012.038 & 1 & 1.691 & 0.008 & 135.2 & 0.3 & & & & & \\
\hline $02429+1754$ & COU23 & 2011.066 & 1 & 1.072 & 0.007 & $138.1^{*}$ & * 0.3 & & & & & \\
\hline $02434+3148$ & A 825 & 2011.030 & 1 & 1.887 & 0.009 & 128.8 & 0.3 & & & & & \\
\hline $02439+2059$ & HU430 & 2011.066 & 1 & 0.689 & 0.020 & $191.8^{*}$ & * 0.8 & & & & & \\
\hline $02439+2538$ & BU306AB & 2011.030 & 1 & 2.953 & 0.015 & $19.1^{*}$ & 0.3 & & & & & \\
\hline $02441+1658$ & HEI97 & 2011.066 & 1 & 0.525 & 0.007 & $291.1^{*}$ & * 0.6 & & & & & \\
\hline $02441+2345$ & TDS2345 & 2012.999 & 1 & - & - & - & - & & $\mathrm{NR}$ & & & \\
\hline $02450+1414$ & HU1046 & 2011.030 & 1 & 0.843 & 0.007 & $122.5^{*}$ & $* 0.3$ & & & & & \\
\hline $02454+5634$ & MLR599 & 2012.038 & 1 & 0.177 & 0.040 & $7.0^{*}$ & 8.0 & & & & & \\
\hline $02459+0714$ & A2024 & 2011.030 & 1 & 1.433 & 0.007 & 54.3 & 0.4 & & & & & \\
\hline $02463+1958$ & A2221 & 2011.030 & 1 & 0.367 & 0.013 & $45.6^{*}$ & 1.5 & & & & & \\
\hline $02469+5744$ & HDS360 & 2012.038 & 1 & 0.328 & 0.007 & 15.2 & 0.5 & & & & & \\
\hline
\end{tabular}




\begin{tabular}{|c|c|c|c|c|c|c|c|c|c|c|c|c|}
\hline WDS & Name & Epoch & Bin. & $\begin{array}{c}\rho \\
(")\end{array}$ & $\begin{array}{l}\sigma_{\rho} \\
(")\end{array}$ & $\begin{array}{c}\theta \\
\left(^{\circ}\right)\end{array}$ & $\begin{array}{l}\sigma_{\theta} \\
\left(^{\circ}\right)\end{array}$ & $\Delta m$ & Notes & Orbit & $\begin{array}{r}\Delta \rho(\mathrm{O}-\mathrm{C}) \\
(")\end{array}$ & $\begin{array}{r}\Delta \theta(\mathrm{O}-\mathrm{C}) \\
\left({ }^{\circ}\right)\end{array}$ \\
\hline $02471+0417$ & HEI624 & 2012.999 & 1 & 0.951 & 0.007 & 66.6 & 0.3 & & & & & \\
\hline $02477+0142$ & A2411AB & 2011.066 & 1 & 0.362 & 0.007 & $279.5^{*}$ & 0.6 & & & & & \\
\hline $02477+0142$ & $\mathrm{~A} 2411 \mathrm{AC}$ & 2011.066 & 1 & 2.106 & 0.011 & 9.2 & 0.3 & & & & & \\
\hline $02478+5219$ & HDS362 & 2012.038 & 1 & - & - & - & - & & $\mathrm{NR}$ & & & \\
\hline $02492+0040$ & A 2412 & 2011.066 & 1 & 0.370 & 0.007 & $104.8^{*}$ & 0.9 & & & & & \\
\hline $02512+0141$ & A2338 & 2011.066 & 1 & 0.150 & 0.040 & 355.9 & 3.1 & & & Doc2016a & 0.03 & 7.3 \\
\hline $02529+5300$ & STF314AB,C & 2011.521 & 1 & 1.547 & 0.013 & 315.9 & 1.1 & & & & & \\
\hline $02529+5300$ & STF314AB,C & 2011.521 & 1 & 1.575 & 0.008 & 314.0 & 2.3 & 0.64 & & & & \\
\hline $02532+0742$ & A2025 & 2011.066 & 1 & 0.215 & 0.007 & 23.9 & 2.0 & & & & & \\
\hline $02544+0946$ & A2341 & 2012.999 & 1 & 1.390 & 0.007 & $5.2^{*}$ & 0.3 & & & & & \\
\hline $02551+3236$ & COU678 & 2011.066 & 1 & 1.320 & 0.007 & $140.5^{*}$ & 0.3 & & & & & \\
\hline $02561+2003$ & COR1Aa,Ab & 2012.999 & 1 & - & - & - & - & & NR & & & \\
\hline $02572+0153$ & A 2413 & 2011.066 & 1 & 0.564 & 0.007 & $160.3^{*}$ & 0.5 & & & Jte2018 & 0.00 & 1.3 \\
\hline $02589+2137$ & BU525 & 2011.066 & 1 & 0.573 & 0.007 & $269.9^{*}$ & 0.4 & & & Rbr2018 & 0.03 & -2.4 \\
\hline $03027+7236$ & A 827 & 2012.038 & 1 & 0.176 & 0.040 & 40.7 & 8.0 & & & & & \\
\hline $03032+7436$ & MLR387 & 2012.038 & 1 & 0.414 & 0.007 & $119.9^{*}$ & 1.4 & 0.70 & & & & \\
\hline $03035+2304$ & HDS389 & 2012.999 & 1 & 0.158 & 0.040 & $351.9^{*}$ & 8.0 & & & Bag2005 & 0.02 & -9.0 \\
\hline $03052+0713$ & A2029 & 2012.999 & 1 & 0.808 & 0.007 & $41.0^{*}$ & 0.7 & & & & & \\
\hline $03054+2515$ & STF346AB & 2011.066 & 1 & 0.463 & 0.007 & $252.1^{*}$ & 0.4 & & & Hei1981a & -0.00 & -4.8 \\
\hline $03069+2052$ & AG61 & 2012.999 & 1 & 0.537 & 0.007 & $23.3^{*}$ & 0.6 & & & & & \\
\hline $03076+5230$ & MLR658 & 2012.038 & 1 & 0.407 & 0.013 & $86.2^{*}$ & 1.2 & 0.80 & & & & \\
\hline $03089+6223$ & HDS400 & 2012.038 & 1 & - & - & - & - & & NR & & & \\
\hline $03090+7635$ & MLR452 & 2012.038 & 1 & 0.601 & 0.010 & $354.5^{*}$ & 0.7 & 1.50 & & & & \\
\hline $03096+0512$ & A2030 & 2012.999 & 1 & 0.280 & 0.007 & $356.3^{*}$ & 0.3 & & & Sta1978b & -0.01 & 0.1 \\
\hline $03119+6131$ & HDS407 & 2012.038 & 1 & 0.522 & 0.007 & $185.7^{*}$ & 0.8 & & & Cve2020b & -0.01 & 2.7 \\
\hline $03126+6914$ & HDS411 & 2012.038 & 1 & 0.395 & 0.038 & 36.0 & 2.2 & & & & & \\
\hline $03143+2257$ & BU530 & 2011.030 & 1 & 1.931 & 0.010 & $192.4^{*}$ & 0.3 & & & & & \\
\hline $03151+1618$ & HU1055 & 2012.999 & 1 & 0.475 & 0.007 & $119.3^{*}$ & 0.3 & & & Hrt2000a & 0.06 & -2.4 \\
\hline $03168+0501$ & A2032 & 2011.030 & 1 & 2.550 & 0.018 & $271.3^{*}$ & 0.3 & & & & & \\
\hline $03169+1715$ & COU361 & 2011.066 & 1 & 1.842 & 0.009 & $17.4^{*}$ & 0.3 & & & & & \\
\hline $03181+0803$ & HDS414 & 2012.999 & 1 & 0.893 & 0.007 & $309.4^{*}$ & 0.8 & & & & & \\
\hline $03209+0110$ & HO320 & 2011.030 & 1 & 2.142 & 0.011 & $173.6^{*}$ & 0.3 & & & & & \\
\hline $03222+6856$ & MLR389 & 2012.030 & 1 & 0.340 & 0.009 & 25.1 & 2.2 & & & & & \\
\hline $03242+2347$ & WOR4 & 2011.030 & 1 & - & - & - & - & & NR & & & \\
\hline $03253+6029$ & A978 & 2011.521 & 1 & 2.412 & 0.023 & 66.0 & 5.3 & & & & & \\
\hline $03253+6029$ & A978 & 2011.521 & 1 & 2.340 & 0.012 & 62.7 & 3.3 & 4.81 & & & & \\
\hline $03291+5732$ & MLR663 & 2012.030 & 1 & 0.631 & 0.007 & $21.5^{*}$ & 0.5 & 1.10 & & & & \\
\hline $03318+0749$ & A1931 & 2011.030 & 1 & 0.862 & 0.008 & 49.0 & 0.6 & & & & & \\
\hline $03336+0705$ & A1932 & 2011.066 & 1 & 0.198 & 0.040 & $227.0^{*}$ & 8.0 & & & & & \\
\hline $03344+2428$ & STF412 & 2011.030 & 1 & 0.742 & 0.007 & $354.5^{*}$ & 2.5 & & & Sca2002a & 0.01 & 1.5 \\
\hline $03344+2428$ & STF412 & 2011.461 & 1 & 0.741 & 0.022 & 353.8 & 2.5 & & & Sca2002a & 0.01 & 1.0 \\
\hline $03350+6002$ & STF400 & 2012.030 & 1 & 1.587 & 0.008 & $267.7^{*}$ & 0.3 & & & Izm2019 & 0.00 & 0.8 \\
\hline $03359+0840$ & HEI451 & 2011.066 & 1 & 1.103 & 0.007 & $248.6^{*}$ & 0.3 & & & & & \\
\hline $03372+0121$ & A2419 & 2011.066 & 1 & 0.835 & 0.010 & $99.8^{*}$ & 0.6 & & & & & \\
\hline $03379+5110$ & COU2573 & 2012.030 & 1 & 0.509 & 0.007 & $136.6^{*}$ & 0.3 & 0.20 & & & & \\
\hline $03399+6017$ & MLR38 & 2012.030 & 1 & 0.150 & 0.040 & $139.8^{*}$ & 8.0 & & & & & \\
\hline $03404+1144$ & HEI316 & 2011.066 & 1 & 0.919 & 0.007 & $278.9^{*}$ & 0.3 & & & & & \\
\hline $03414+1650$ & HEI100 & 2011.066 & 1 & 0.860 & 0.007 & $166.1^{*}$ & 0.3 & & & & & \\
\hline $03426+0838$ & HDS472AB & 2011.066 & 1 & 0.565 & 0.007 & $245.4^{*}$ & 0.3 & & & & & \\
\hline $03426+0838$ & HDS472AC & 2011.066 & 1 & 4.179 & 0.021 & 262.7 & 0.3 & 0.71 & & & & \\
\hline $03427+6950$ & STF419AB & 2012.030 & 1 & 2.891 & 0.014 & $72.8^{*}$ & 0.3 & & & & & \\
\hline $03427+6950$ & A984BC & 2012.030 & 1 & 0.418 & 0.037 & 95.1 & 2.3 & 1.39 & & & & \\
\hline $03427+6950$ & A984BC & 2012.030 & 1 & 0.438 & 0.037 & $97.6^{*}$ & 2.3 & & & & & \\
\hline $03444+2030$ & COU261 & 2011.066 & 1 & 1.179 & 0.007 & $241.9^{*}$ & 0.3 & & & & & \\
\hline
\end{tabular}




\begin{tabular}{|c|c|c|c|c|c|c|c|c|c|c|c|c|}
\hline WDS & Name & Epoch & Bin. & $\begin{array}{c}\rho \\
(")\end{array}$ & $\begin{array}{l}\sigma_{\rho} \\
(")\end{array}$ & $\begin{array}{c}\theta \\
\left(^{\circ}\right)\end{array}$ & $\begin{array}{l}\sigma_{\theta} \\
\left(^{\circ}\right)\end{array}$ & $\Delta m$ & Notes & Orbit & $\begin{array}{r}\Delta \rho(\mathrm{O}-\mathrm{C}) \\
(")\end{array}$ & $\begin{array}{r}\Delta \theta(\mathrm{O}-\mathrm{C}) \\
\left({ }^{\circ}\right)\end{array}$ \\
\hline $03456+2839$ & COU692 & 2011.066 & 1 & 1.712 & 0.009 & $318.4^{*}$ & 0.3 & & & & & \\
\hline $03460+7302$ & MLR457 & 2012.030 & 1 & 0.934 & 0.007 & $4.5^{*}$ & 0.3 & & & & & \\
\hline $03480+6840$ & KUI13BC & 2012.030 & 1 & 0.763 & 0.007 & $26.3^{*}$ & 0.3 & 0.20 & & & & \\
\hline $03486+2411$ & BU1105 & 2011.066 & 1 & 0.193 & 0.040 & 40.5 & 8.0 & & & & & \\
\hline $03503+2535$ & STT65 & 2011.066 & 1 & 0.280 & 0.007 & $199.5^{*}$ & 0.8 & & & DRs2012 & -0.01 & 1.3 \\
\hline $03522+5357$ & MLR665 & 2012.038 & 1 & 0.355 & 0.008 & $154.4^{*}$ & 0.6 & 0.50 & & & & \\
\hline $03544+5839$ & HDS490 & 2012.030 & 1 & - & - & - & - & & NR & & & \\
\hline $03562+5939$ & HDS497Aa,Ab & 2012.038 & 1 & 0.480 & 0.007 & 85.5 & 0.4 & & & & & \\
\hline $03570+5427$ & MLR659 & 2012.030 & 1 & 0.618 & 0.007 & $158.2^{*}$ & 0.6 & 0.70 & & & & \\
\hline $03589+7152$ & A994 & 2012.030 & 1 & 0.723 & 0.007 & $15.3^{*}$ & 0.3 & 0.40 & & & & \\
\hline $03590+0947$ & HU27 & 2011.066 & 1 & 0.427 & 0.013 & $333.9^{*}$ & 0.3 & & & Hrt2010a & 0.02 & 4.1 \\
\hline $04017+5611$ & HDS507 & 2012.038 & 1 & 0.538 & 0.011 & $134.9^{*}$ & 0.5 & 0.10 & & & & \\
\hline $04021+6417$ & HEI638 & 2012.870 & 1 & 1.829 & 0.009 & $257.6^{*}$ & 0.3 & & & & & \\
\hline $04027+5209$ & HU210 & 2012.030 & 1 & - & - & - & - & & NR & & & \\
\hline $04029+6225$ & HDS512 & 2012.038 & 1 & 0.437 & 0.007 & $182.1^{*}$ & 1.2 & & & & & \\
\hline $04038+6212$ & MLR110 & 2012.030 & 1 & 0.973 & 0.007 & $259.6^{*}$ & 0.3 & & & & & \\
\hline $04038+6212$ & MLR110 & 2012.038 & 1 & 0.973 & 0.007 & $259.4^{*}$ & 0.3 & & & & & \\
\hline $04054+2309$ & COU563 & 2011.066 & 1 & 0.393 & 0.023 & $105.6^{*}$ & 1.4 & & & & & \\
\hline $04068+7110$ & MLR458 & 2012.030 & 1 & 0.577 & 0.018 & $265.9^{*}$ & 1.4 & & & & & \\
\hline $04102+1722$ & HEI35 & 2011.066 & 1 & 0.417 & 0.008 & $334.8^{*}$ & 1.2 & & & Tok2021 & -0.04 & 1.6 \\
\hline $04199+1631$ & STT79 & 2011.066 & 1 & 0.479 & 0.007 & $173.9^{*}$ & 0.3 & & & Sod1999 & -0.02 & $-1.7^{Q}$ \\
\hline $04204+7355$ & MLR397 & 2012.030 & 1 & 0.191 & 0.040 & 171.2 & 8.0 & & & & & \\
\hline $04212+0614$ & A1834 & 2011.066 & 1 & 0.461 & 0.007 & $283.2^{*}$ & 1.1 & & & & & \\
\hline $04216+0658$ & A1835 & 2011.066 & 1 & 0.188 & 0.040 & $3.6^{*}$ & 8.0 & & & Zir2010 & -0.02 & -16.3 \\
\hline $04230+5937$ & KUI16 & 2012.030 & 1 & 1.247 & 0.007 & $200.5^{*}$ & 0.3 & & & & & \\
\hline $04232+7510$ & MLR459 & 2012.030 & 1 & 0.404 & 0.007 & $109.8^{*}$ & 0.3 & 0.20 & & & & \\
\hline $04233+1123$ & STF535 & 2011.066 & 1 & 1.143 & 0.007 & $272.8^{*}$ & 0.3 & & & Izm2019 & -0.00 & -0.5 \\
\hline $04233+1123$ & STF535 & 2011.066 & 1 & 1.134 & 0.015 & 272.6 & 0.4 & & & Izm2019 & 0.01 & -0.3 \\
\hline $04274+5218$ & COU2360 & 2012.038 & 1 & 0.804 & 0.007 & $13.2^{*}$ & 0.3 & 0.60 & & & & \\
\hline $04280+6709$ & HDS573 & 2012.038 & 1 & 0.677 & 0.021 & 110.7 & 0.7 & & & & & \\
\hline $04301+1538$ & STF554 & 2011.113 & 1 & 1.539 & 0.030 & 15.9 & 0.7 & & & Trr2019 & -0.01 & 1.1 \\
\hline $04301+1538$ & STF554 & 2011.113 & 1 & 1.532 & 0.008 & 17.5 & 0.3 & & & Trr2019 & -0.00 & -0.4 \\
\hline $04302+5343$ & A1300 & 2012.038 & 1 & 0.876 & 0.007 & $150.1^{*}$ & 0.3 & & & & & \\
\hline $04306+3227$ & STT83A,BC & 2012.362 & 1 & 5.584 & 0.028 & 129.4 & 0.3 & 4.03 & & & & \\
\hline $04308+6427$ & HDS581 & 2012.038 & 1 & - & - & - & - & & NR & & & \\
\hline $04334+1949$ & A2351AB & 2011.113 & 1 & 1.010 & 0.011 & 174.4 & 0.8 & & & & & \\
\hline $04334+1949$ & A2351AB & 2011.113 & 1 & 1.004 & 0.007 & 174.0 & 0.4 & & & & & \\
\hline $04334+1949$ & $\mathrm{~A} 2351 \mathrm{AC}$ & 2011.113 & 1 & 3.076 & 0.015 & 173.1 & 0.3 & 3.18 & ND & & & \\
\hline $04341+7604$ & MLR462 & 2012.038 & 1 & 0.953 & 0.007 & $173.3^{*}$ & 0.4 & 1.50 & & & & \\
\hline $04383+7853$ & MLR463 & 2012.038 & 1 & 0.195 & 0.040 & 165.5 & 8.0 & & & & & \\
\hline $04422+3731$ & STF577 & 2011.134 & 1 & 0.747 & 0.027 & 340.6 & 1.3 & & & RAO2015 & 0.02 & 1.0 \\
\hline $04441+0601$ & HEI841 & 2011.134 & 1 & 0.723 & 0.010 & 30.2 & 1.6 & & & & & \\
\hline $04449+5026$ & COU2460 & 2012.030 & 1 & 0.469 & 0.007 & $304.5^{*}$ & 0.4 & 0.80 & & & & \\
\hline $04453+6340$ & MLR124 & 2012.030 & 1 & 0.692 & 0.007 & $250.1^{*}$ & 0.3 & & & & & \\
\hline $04457+6141$ & MLR111 & 2012.030 & 1 & 0.125 & 0.040 & 84.0 & 8.0 & & & & & \\
\hline $04481+7810$ & MLR464 & 2012.030 & 1 & 1.218 & 0.008 & $204.4^{*}$ & 0.4 & 0.50 & & & & \\
\hline $04486+6303$ & MLR39 & 2012.030 & 1 & 0.368 & 0.007 & $128.1^{*}$ & 0.4 & 0.20 & & & & \\
\hline $04529+7543$ & MLR465 & 2012.030 & 1 & 0.928 & 0.007 & $220.0^{*}$ & 0.3 & & & & & \\
\hline $04539+5603$ & HU818 & 2012.038 & 1 & 0.466 & 0.007 & $72.2^{*}$ & 0.3 & 0.50 & & & & \\
\hline $04560+5619$ & HDS638 & 2012.038 & 1 & 0.132 & 0.040 & 125.9 & 8.0 & & & & & \\
\hline $04586+6725$ & MLR398 & 2012.030 & 1 & 0.193 & 0.040 & 168.7 & 8.0 & & & & & \\
\hline $04591+6322$ & HU1090 & 2012.030 & 1 & 0.206 & 0.010 & $290.8^{*}$ & 0.5 & 0.10 & & Msn2014b & 0.00 & 6.8 \\
\hline $04598+7542$ & MLR466 & 2012.038 & 1 & 0.338 & 0.017 & $216.4^{*}$ & 0.7 & 0.20 & & & & \\
\hline
\end{tabular}




\begin{tabular}{|c|c|c|c|c|c|c|c|c|c|c|c|c|}
\hline WDS & Name & Epoch & Bin. & $\begin{array}{c}\rho \\
(")\end{array}$ & $\begin{array}{l}\sigma_{\rho} \\
(")\end{array}$ & $\begin{array}{c}\theta \\
\left({ }^{\circ}\right)\end{array}$ & $\begin{array}{l}\sigma_{\theta} \\
\left({ }^{\circ}\right)\end{array}$ & $\Delta m$ & Notes & Orbit & $\begin{array}{r}\Delta \rho(\mathrm{O}-\mathrm{C}) \\
(")\end{array}$ & $\begin{array}{r}\Delta \theta(\mathrm{O}-\mathrm{C}) \\
\left({ }^{\circ}\right)\end{array}$ \\
\hline $04598+7542$ & MLR466 & 2012.030 & 1 & 0.349 & 0.019 & 216.7 & 0.3 & & & & & \\
\hline $05001+6958$ & MLR399 & 2012.030 & 1 & 0.279 & 0.007 & $345.8^{*}$ & 0.5 & 0.40 & & & & \\
\hline $05005+0506$ & STT93 & 2011.134 & 1 & 1.544 & 0.008 & 244.0 & 0.3 & & & WSI2015 & 0.02 & -0.0 \\
\hline $05012+3430$ & COU1229 & 2011.143 & 1 & 1.745 & 0.018 & 47.0 & 0.5 & & & & & \\
\hline $05012+3430$ & COU1229 & 2012.000 & 1 & 1.713 & 0.021 & 46.5 & 0.4 & & & & & \\
\hline $05014+0852$ & A2041 & 2011.143 & 1 & 0.821 & 0.024 & 119.0 & 2.5 & & & & & \\
\hline $05017+2640$ & A1844 & 2011.143 & 1 & 0.363 & 0.041 & 13.3 & 2.4 & & & Msn2005 & 0.03 & 3.4 \\
\hline $05017+2640$ & A1844 & 2011.143 & 1 & 0.310 & 0.034 & 10.8 & 2.5 & & & Msn2005 & -0.02 & 0.9 \\
\hline $05020+0137$ & A2630 & 2011.134 & 1 & 0.213 & 0.024 & 172.6 & 3.6 & & & & & \\
\hline $05036+7935$ & MLR491 & 2012.038 & 1 & 0.623 & 0.007 & $66.6^{*}$ & 0.5 & & & & & \\
\hline $05041+0257$ & A 2632 & 2011.134 & 1 & 0.894 & 0.007 & 123.8 & 0.7 & & & & & \\
\hline $05047+7404$ & STT89 & 2012.030 & 1 & 0.326 & 0.010 & $306.0^{*}$ & 1.0 & 0.80 & & Alz1998a & 0.06 & -0.4 \\
\hline $05058+0811$ & A1945 & 2011.134 & 1 & 0.153 & 0.040 & 42.3 & 8.0 & & & & & \\
\hline $05100+7541$ & A841 & 2012.038 & 1 & 0.766 & 0.007 & $236.4^{*}$ & 0.3 & 1.00 & & & & \\
\hline $05166+5934$ & MLR563 & 2012.038 & 1 & - & - & - & - & & NR & & & \\
\hline $05198+7009$ & MLR401 & 2012.038 & 1 & 1.170 & 0.007 & $103.4^{*}$ & 0.3 & & & & & \\
\hline $05212+3235$ & COU1232 & 2011.143 & 1 & 1.128 & 0.017 & 65.1 & 0.5 & & & & & \\
\hline $05219+3934$ & COU2037 & 2011.143 & 1 & 0.346 & 0.010 & 143.6 & 1.9 & & & & & \\
\hline $05226+0236$ & A2641 & 2011.143 & 1 & 1.116 & 0.054 & 158.0 & 1.3 & & & Izm2019 & 0.01 & 1.1 \\
\hline $05227+0008$ & A2642 & 2011.143 & 1 & 0.519 & 0.015 & 59.3 & 3.0 & & & & & \\
\hline $05237+7347$ & $\mathrm{~A} 843 \mathrm{AB}$ & 2012.038 & 1 & - & - & - & - & & $\mathrm{NR}$ & & & \\
\hline $05239-0052$ & WNC2A,BC & 2011.143 & 1 & 3.077 & 0.025 & 159.9 & 0.3 & & & & & \\
\hline $05239-0052$ & WNC2A,BC & 2011.143 & 1 & 3.073 & 0.015 & 160.0 & 0.3 & & & & & \\
\hline $05249+5347$ & MLR667 & 2012.038 & 1 & - & - & - & - & & NR & & & \\
\hline $05271+5725$ & MLR600 & 2012.038 & 1 & - & - & - & - & & NR & & & \\
\hline $05296+0309$ & BU557BC & 2011.143 & 1 & 0.288 & 0.038 & 22.6 & 2.5 & & & & & \\
\hline $05297+3523$ & HU217 & 2011.134 & 1 & 0.577 & 0.023 & 73.4 & 3.0 & & & & & \\
\hline $05357+3654$ & COU2042 & 2011.134 & 1 & 1.588 & 0.010 & 116.7 & 0.3 & & & & & \\
\hline $05360+1933$ & HDS749 & 2011.135 & 1 & 0.814 & 0.030 & 70.7 & 1.2 & & & & & \\
\hline $05372+7928$ & MLR492 & 2012.038 & 1 & 1.351 & 0.007 & $125.4^{*}$ & 0.3 & 0.20 & & & & \\
\hline $05373+6642$ & MLR314 & 2012.038 & 1 & 0.209 & 0.040 & 290.2 & 8.0 & & & Doc2017c & -0.02 & 12.8 \\
\hline $05379+0857$ & A2708 & 2011.135 & 1 & 0.589 & 0.020 & 139.8 & 0.6 & & & & & \\
\hline $05450+7156$ & MLR403 & 2012.038 & 1 & 0.452 & 0.007 & 62.1 & 1.3 & 0.20 & & & & \\
\hline $05458+5143$ & COU2591 & 2012.038 & 1 & 0.605 & 0.007 & $263.2^{*}$ & 0.3 & & & & & \\
\hline $05506-0126$ & BU1188AB & 2011.143 & 1 & 1.024 & 0.012 & 91.2 & 1.0 & & & & & \\
\hline $05513-0634$ & RST4287AB & 2011.143 & 1 & 1.342 & 0.026 & 33.2 & 0.5 & & & & & \\
\hline $05537-0542$ & HDS793 & 2011.143 & 1 & 0.542 & 0.016 & 123.0 & 2.5 & & & & & \\
\hline $05551+1506$ & HEI111 & 2011.135 & 1 & 0.123 & 0.047 & 177.7 & 21.6 & & & & & \\
\hline $05551+7646$ & MLR469 & 2012.038 & 1 & 0.224 & 0.009 & $101.6^{*}$ & 2.2 & 0.20 & & & & \\
\hline $05557+7755$ & HDS798 & 2012.038 & 1 & 0.688 & 0.007 & $59.2^{*}$ & 0.5 & & & & & \\
\hline $05592+7749$ & MLR470 & 2012.038 & 1 & 0.979 & 0.007 & $174.6^{*}$ & 0.3 & 0.50 & & & & \\
\hline $06004+1156$ & TDS213 & 2011.135 & 1 & 1.043 & 0.023 & 177.0 & 2.1 & & & & & \\
\hline $06032+5813$ & A1315 & 2012.038 & 2 & 0.850 & 0.010 & $87.4^{*}$ & 0.9 & 0.20 & & Doc1984b & -0.05 & $-1.8^{Q}$ \\
\hline $06041+1101$ & J335 & 2012.142 & 1 & 1.260 & 0.007 & $271.2^{*}$ & 0.3 & & & Ole2002a & 0.06 & -4.2 \\
\hline $06045+2208$ & COU162 & 2011.135 & 1 & 1.070 & 0.021 & 133.5 & 0.3 & & & & & \\
\hline $06049+3211$ & HU827 & 2012.142 & 1 & 0.250 & 0.008 & $75.6^{*}$ & 0.7 & & & & & \\
\hline $06060-6518$ & HDS831 & 2011.560 & 1 & 0.314 & 0.021 & $60.6^{*}$ & 2.4 & & & & & \\
\hline $06074+3616$ & STT131 & 2011.178 & 1 & 1.508 & 0.018 & 96.2 & 1.4 & & & & & \\
\hline $06075+0755$ & A1953 & 2011.178 & 1 & 0.340 & 0.008 & 75.3 & 2.1 & & & & & \\
\hline $06075+0755$ & A1953 & 2012.000 & 1 & 0.330 & 0.044 & 77.1 & 2.4 & & & & & \\
\hline $06097+1630$ & A 2514 & 2011.135 & 1 & 0.226 & 0.080 & 113.2 & 19.4 & & & & & \\
\hline
\end{tabular}




\begin{tabular}{|c|c|c|c|c|c|c|c|c|c|c|c|c|}
\hline WDS & Name & Epoch & Bin. & $\begin{array}{c}\rho \\
(")\end{array}$ & $\begin{array}{l}\sigma_{\rho} \\
(")\end{array}$ & $\begin{array}{c}\theta \\
\left({ }^{\circ}\right)\end{array}$ & $\begin{array}{l}\sigma_{\theta} \\
\left(^{\circ}\right)\end{array}$ & $\Delta m$ & Notes & Orbit & $\begin{array}{r}\Delta \rho(\mathrm{O}-\mathrm{C}) \\
(")\end{array}$ & $\begin{array}{r}\Delta \theta(\mathrm{O}-\mathrm{C}) \\
\left({ }^{\circ}\right)\end{array}$ \\
\hline $06097+1630$ & A2514 & 2011.135 & 1 & 0.258 & 0.039 & 104.5 & 1.2 & & & & & \\
\hline $06097+1630$ & A 2514 & 2011.135 & 1 & 0.241 & 0.066 & 109.2 & 15.0 & & & & & \\
\hline $06145+1754$ & KUI24 & 2011.135 & 1 & 0.339 & 0.009 & 144.0 & 1.7 & & & Zir2013a & 0.01 & 0.8 \\
\hline $06145+6458$ & HDS854 & 2012.038 & 1 & 0.275 & 0.007 & $192.3^{*}$ & ${ }^{*} 0.4$ & 0.30 & & & & \\
\hline $06156+6609$ & MLR316 & 2012.038 & 1 & 0.271 & 0.010 & $314.9^{*}$ & ${ }^{*} 0.6$ & 0.50 & & & & \\
\hline $06171+0957$ & FIN331 & 2011.178 & 1 & 0.360 & 0.021 & 80.1 & 2.3 & & & & & \\
\hline $06186+2151$ & HU451 & 2011.178 & 1 & 0.549 & 0.011 & 168.0 & 1.0 & & & & & \\
\hline $06203+0743$ & A2719 & 2011.143 & 1 & 0.334 & 0.018 & 59.5 & 2.9 & & & & & \\
\hline $06207+1832$ & A 2516 & 2011.143 & 1 & 0.751 & 0.022 & 108.3 & 1.0 & & & & & \\
\hline $06209+3250$ & COU1542 & 2012.142 & 1 & 1.053 & 0.007 & $187.6^{*}$ & ${ }^{*} 0.3$ & 0.80 & & & & \\
\hline $06218+1317$ & HEI469 & 2012.142 & 1 & 1.138 & 0.007 & $176.7^{*}$ & ${ }^{*} 0.4$ & & & & & \\
\hline $06240+2017$ & HU703 & 2012.142 & 1 & 0.407 & 0.007 & $33.8^{*}$ & 0.3 & & & & & \\
\hline $06243+3207$ & COU1543 & 2012.142 & 1 & 1.447 & 0.016 & $103.9^{*}$ & 0.4 & & & & & \\
\hline $06253+0801$ & $\mathrm{~A} 2812 \mathrm{~A}, \mathrm{BC}$ & 2012.142 & 2 & 9.889 & 0.049 & 56.9 & 0.3 & & & & & \\
\hline $06253+0801$ & $\mathrm{~A} 2812 \mathrm{AB}$ & 2012.142 & 1 & 0.561 & 0.007 & $190.5^{*}$ & 0.3 & & & & & \\
\hline $06283+2441$ & COU914AB & 2012.142 & 2 & - & - & - & - & & NR & & & \\
\hline $06283+2441$ & COU914AC & 2012.142 & 2 & 8.911 & 0.045 & $28.8^{*}$ & 0.3 & & ND & & & \\
\hline $06317+2823$ & BU1021 & 2012.197 & 1 & 0.694 & 0.007 & $75.9^{*}$ & 0.9 & & & & & \\
\hline $06317+2823$ & BU1021 & 2012.199 & 1 & 0.694 & 0.007 & $74.6^{*}$ & 0.3 & & & & & \\
\hline $06318+1434$ & HEI329 & 2012.197 & 1 & 0.303 & 0.027 & $325.6^{*}$ & 2.7 & & & & & \\
\hline $06318+1434$ & HEI329 & 2012.199 & 1 & 0.328 & 0.029 & 329.9 & 6.0 & & & & & \\
\hline $06323+1542$ & STT145 & 2012.199 & 1 & 1.360 & 0.007 & $336.0^{*}$ & 0.3 & & & & & \\
\hline $06323+5225$ & WOR6 & 2012.202 & 1 & 0.771 & 0.007 & $159.6^{*}$ & 0.4 & & & Hrt2009 & 0.02 & 3.0 \\
\hline $06330+5547$ & MLR669 & 2012.202 & 1 & 0.995 & 0.007 & $46.5^{*}$ & 0.3 & & & & & \\
\hline $06336+1032$ & A2818 & 2012.199 & 1 & 1.432 & 0.007 & $343.4^{*}$ & 0.3 & 0.20 & & & & \\
\hline $06344+1445$ & STF932 & 2012.197 & 1 & 1.655 & 0.008 & $306.0^{*}$ & 0.3 & & & Hop1960a & 0.02 & 4.7 \\
\hline $06350+0816$ & HDS899 & 2012.197 & 1 & 0.392 & 0.024 & 50.9 & 2.1 & & & & & \\
\hline $06352+3348$ & COU1545 & 2011.184 & 1 & 0.744 & 0.030 & 74.0 & 0.5 & & & & & \\
\hline $06358+3621$ & COU1875 & 2011.184 & 1 & 1.332 & 0.015 & 150.9 & 0.3 & & & & & \\
\hline $06363+1536$ & HEI119 & 2012.199 & 1 & 1.094 & 0.009 & $149.6^{*}$ & 1.5 & & & & & \\
\hline $06366+7756$ & HDS905 & 2012.202 & 1 & 0.455 & 0.014 & $316.0^{*}$ & 0.9 & & & & & \\
\hline $06375+2435$ & HDS910 & 2011.184 & 1 & 0.486 & 0.007 & 61.9 & 0.7 & & & & & \\
\hline $06380+1442$ & HEI220 & 2012.199 & 1 & 0.856 & 0.008 & $139.8^{*}$ & 0.7 & & & & & \\
\hline $06381+5927$ & HDS916BC & 2012.202 & 1 & - & - & - & - & & NR & & & \\
\hline $06385+1020$ & HEI702 & 2012.199 & 1 & 0.822 & 0.008 & $42.2^{*}$ & 0.6 & & & & & \\
\hline $06389+3515$ & COU1550 & 2012.142 & 2 & 1.340 & 0.025 & $213.3^{*}$ & 0.7 & & & & & \\
\hline $06389+3515$ & COU1550 & 2011.184 & 1 & 1.314 & 0.025 & 213.5 & 1.0 & & & & & \\
\hline $06396+2816$ & STT152 & 2012.200 & 1 & 0.865 & 0.007 & $34.8^{*}$ & 0.3 & & & & & \\
\hline $06404+2505$ & A1053 & 2011.135 & 1 & 1.024 & 0.018 & 155.2 & 1.3 & & & & & \\
\hline $06406+2314$ & COU86 & 2011.184 & 1 & 1.133 & 0.012 & 36.5 & 0.3 & & & & & \\
\hline $06410+2045$ & COU582 & 2011.184 & 1 & 1.088 & 0.007 & 151.9 & 0.9 & & & & & \\
\hline $06418+3041$ & A 218 & 2011.135 & 1 & 0.160 & 0.060 & 39.0 & 8.0 & & & Msn2017a & -0.08 & 0.9 \\
\hline $06418+3041$ & A 218 & 2011.135 & 1 & 0.232 & 0.048 & 30.9 & 6.3 & & & Msn2017a & -0.01 & -7.2 \\
\hline $06422+7429$ & HDS929 & 2012.202 & 1 & 0.176 & 0.040 & $194.4^{*}$ & 8.0 & & & & & \\
\hline $06425+7035$ & MLR405 & 2012.202 & 1 & 0.371 & 0.007 & $256.4^{*}$ & 0.6 & & & & & \\
\hline $06439+1533$ & HEI120 & 2012.200 & 1 & 1.007 & 0.007 & $259.0^{*}$ & 1.3 & & & & & \\
\hline $06439+1533$ & HEI120 & 2011.143 & 1 & 1.009 & 0.022 & 258.1 & 1.3 & & & & & \\
\hline $06442+0612$ & A2729 & 2011.143 & 1 & 0.410 & 0.035 & 139.1 & 4.4 & & & & & \\
\hline $06442+0612$ & A2729 & 2012.000 & 1 & 0.315 & 0.048 & 139.3 & 2.9 & & & & & \\
\hline $06452+0941$ & A2827 & 2012.142 & 2 & 0.739 & 0.015 & $176.9^{*}$ & 0.8 & & & & & \\
\hline $06452+2913$ & COU1240 & 2012.142 & 2 & 0.490 & 0.015 & 86.7 & 0.3 & & & & & \\
\hline $06455+2922$ & A122 & 2011.135 & 1 & 0.441 & 0.008 & 26.7 & 0.3 & & & Sca2020d & -0.01 & -0.8 \\
\hline $06455+2922$ & A122 & 2011.135 & 1 & 0.441 & 0.009 & 29.3 & 1.5 & & & Sca2020d & -0.01 & 1.8 \\
\hline
\end{tabular}




\begin{tabular}{|c|c|c|c|c|c|c|c|c|c|c|c|c|}
\hline WDS & Name & Epoch & Bin. & $\begin{array}{c}\rho \\
(")\end{array}$ & $\begin{array}{l}\sigma_{\rho} \\
(")\end{array}$ & $\begin{array}{c}\theta \\
\left({ }^{\circ}\right)\end{array}$ & $\begin{array}{l}\sigma_{\theta} \\
\left({ }^{\circ}\right)\end{array}$ & $\Delta m$ & Notes & Orbit & $\begin{array}{r}\Delta \rho(\mathrm{O}-\mathrm{C}) \\
(")\end{array}$ & $\begin{array}{r}\Delta \theta(\mathrm{O}-\mathrm{C}) \\
\left({ }^{\circ}\right)\end{array}$ \\
\hline $06456+5420$ & A1735 & 2012.202 & 1 & 0.322 & 0.007 & $240.4^{*}$ & 0.4 & & & & & \\
\hline $06463+1812$ & HO238 & 2011.135 & 1 & 0.368 & 0.007 & 170.5 & 2.0 & & & & & \\
\hline $06467+1755$ & COU916 & 2011.135 & 1 & 1.646 & 0.014 & 68.3 & 0.4 & & & & & \\
\hline $06470+7347$ & MLR475 & 2012.202 & 1 & 0.459 & 0.007 & $2.3^{*}$ & 1.2 & & & & & \\
\hline $06471+1001$ & A 2828 & 2012.142 & 2 & - & - & - & - & & NR & & & \\
\hline $06477+2338$ & COU87 & 2012.142 & 2 & 0.391 & 0.018 & 96.6 & 2.8 & & & & & \\
\hline $06485+0351$ & A1955 & 2012.142 & 2 & - & - & - & - & & $\mathrm{NR}$ & & & \\
\hline $06487+0737$ & $\mathrm{~A} 2731 \mathrm{AB}$ & 2011.143 & 1 & 1.304 & 0.019 & 65.8 & 0.5 & & & Izm2019 & -0.02 & 0.4 \\
\hline $06489+0707$ & $\mathrm{~A} 2732$ & 2011.143 & 1 & 0.305 & 0.007 & 122.7 & 1.3 & & & & & \\
\hline $06494+6425$ & MLR566 & 2012.202 & 1 & 0.227 & 0.007 & $246.2^{*}$ & 1.6 & & & & & \\
\hline $06539+6945$ & MLR406 & 2012.202 & 1 & 0.921 & 0.007 & $118.2^{*}$ & 0.3 & & & & & \\
\hline $06552+2721$ & COU946 & 2011.184 & 1 & 0.924 & 0.025 & 77.2 & 1.4 & & & & & \\
\hline $06555+3010$ & STF981 & 2011.184 & 1 & 1.071 & 0.008 & 123.6 & 0.7 & & & Kiy2017 & 0.01 & 0.9 \\
\hline $06560+6514$ & MLR132 & 2012.202 & 1 & 1.208 & 0.007 & $222.8^{*}$ & 0.3 & & & & & \\
\hline $06575+0253$ & A2681 & 2011.143 & 1 & 0.448 & 0.008 & 318.3 & 2.4 & & & USN2007b & 0.04 & 3.2 \\
\hline $06582+6516$ & MLR133Aa,Ab & 2012.202 & 1 & - & - & - & - & & NR & & & \\
\hline $06592+1843$ & BU899 & 2012.197 & 1 & 0.742 & 0.007 & $263.2^{*}$ & 0.3 & & & & & \\
\hline $06594+1308$ & HEI336 & 2012.197 & 1 & 1.342 & 0.007 & $341.4^{*}$ & 0.3 & & & & & \\
\hline $07002+0900$ & A 2838 & 2012.156 & 2 & 2.015 & 0.015 & $11.8^{*}$ & 0.3 & & & & & \\
\hline $07008+2716$ & BU1022 & 2012.197 & 1 & 0.355 & 0.007 & $216.0^{*}$ & 1.3 & & & & & \\
\hline $07012+1146$ & STT163AB & 2011.143 & 1 & 0.233 & 0.069 & 112.4 & 4.0 & & & Zul1996b & 0.01 & -0.2 \\
\hline $07012+1146$ & STT163AB & 2011.143 & 1 & 0.200 & 0.026 & 115.1 & 2.8 & & & Zul1996b & -0.02 & 2.5 \\
\hline $07013+0612$ & TDS4390 & 2012.156 & 2 & - & - & - & - & & NR & & & \\
\hline $07015+1009$ & HEI717 & 2011.184 & 1 & 1.052 & 0.012 & 168.0 & 0.4 & & & & & \\
\hline $07017+1220$ & HEI470 & 2012.156 & 2 & 0.472 & 0.015 & $99.6^{*}$ & 1.7 & & & & & \\
\hline $07052+7039$ & HDS987 & 2012.202 & 1 & 0.723 & 0.007 & $194.1^{*}$ & 0.4 & & & & & \\
\hline $07054+2444$ & A1065 & 2012.197 & 1 & 0.183 & 0.040 & $142.6^{*}$ & 8.0 & & & & & \\
\hline $07066+3132$ & COU1106 & 2011.135 & 1 & 1.556 & 0.040 & 75.3 & 2.5 & & & & & \\
\hline $07066+3132$ & COU1106 & 2011.184 & 1 & 1.468 & 0.181 & 72.8 & 3.4 & & & & & \\
\hline $07078+1130$ & A 2843 & 2012.197 & 1 & 0.812 & 0.007 & $99.1^{*}$ & 0.7 & & & & & \\
\hline $07082+6339$ & HDS990 & 2012.202 & 1 & 0.184 & 0.040 & 8.8 & 8.0 & & & & & \\
\hline $07082+6339$ & HDS990 & 2012.202 & 1 & - & - & - & - & & NR & & & \\
\hline $07085+1040$ & A 2844 & 2011.184 & 1 & 0.318 & 0.016 & 43.4 & 3.7 & & & & & \\
\hline $07089+1412$ & HEI126 & 2011.184 & 1 & 1.292 & 0.012 & 177.0 & 0.8 & & & & & \\
\hline $07104+0618$ & A 2846 & 2012.142 & 1 & 0.399 & 0.007 & 49.9 & 0.9 & & & & & \\
\hline $07104+0618$ & A 2846 & 2012.197 & 1 & - & - & - & - & & NR & & & \\
\hline $07134+1610$ & HDS1003 & 2011.135 & 1 & 2.152 & 0.034 & 7.2 & 0.9 & & & & & \\
\hline $07138+2830$ & STT520 & 2012.197 & 1 & 0.568 & 0.007 & $29.6^{*}$ & 0.3 & & & $\mathrm{PkO} 2018 \mathrm{~b}$ & -0.04 & 1.6 \\
\hline $07141+0220$ & HEI724 & 2011.143 & 1 & 0.645 & 0.041 & 60.5 & 2.0 & & & & & \\
\hline $07150+3731$ & COU1882 & 2012.142 & 1 & 0.903 & 0.007 & $61.4^{*}$ & 0.3 & & & & & \\
\hline $07154+1414$ & TDS4633AC & 2012.156 & 2 & 3.879 & 0.021 & 70.9 & 0.4 & & & & & \\
\hline $07162+5618$ & MLR567 & 2012.202 & 1 & 0.187 & 0.040 & 20.8 & 8.0 & & & & & \\
\hline $07164+1227$ & A 2853 & 2012.197 & 1 & 0.483 & 0.007 & $132.3^{*}$ & 0.4 & & & & & \\
\hline $07166+0920$ & A 2854 & 2012.197 & 1 & 0.101 & 0.08 & 7.8 & 7.0 & & & & & \\
\hline $07171+0447$ & TDS4674 & 2012.156 & 2 & 0.889 & 0.022 & $181.8^{*}$ & 0.9 & & & & & \\
\hline $07173+3744$ & COU1883 & 2012.142 & 1 & 0.685 & 0.010 & $242.6^{*}$ & 0.6 & & & & & \\
\hline $07175+1324$ & A 2856 & 2012.156 & 2 & 0.567 & 0.055 & $132.8^{*}$ & 2.6 & & & & & \\
\hline $07175+1324$ & A 2856 & 2012.197 & 1 & 0.497 & 0.057 & 128.1 & 3.5 & & & & & \\
\hline $07182+2157$ & COU276 & 2011.135 & 1 & 1.052 & 0.034 & 49.7 & 1.5 & & & & & \\
\hline $07190+3804$ & COU2069 & 2012.142 & 1 & 0.350 & 0.007 & $106.5^{*}$ & 0.5 & & & & & \\
\hline $07192+5908$ & HDS1018 & 2012.202 & 1 & 0.131 & 0.040 & 62.0 & 8.0 & & & & & \\
\hline $07214+0908$ & A 2940 & 2012.156 & 2 & 1.232 & 0.094 & $181.2^{*}$ & 0.9 & & & & & \\
\hline $07219+0838$ & HEI726 & 2012.156 & 2 & 0.967 & 0.055 & $168.8^{*}$ & ${ }^{k} 1.5$ & & & & & \\
\hline $07229+7407$ & MLR477 & 2012.202 & 1 & 0.235 & 0.010 & $102.2^{*}$ & 0.9 & & & & & \\
\hline
\end{tabular}




\begin{tabular}{|c|c|c|c|c|c|c|c|c|c|c|c|c|}
\hline WDS & Name & Epoch & Bin. & $\begin{array}{c}\rho \\
(")\end{array}$ & $\begin{array}{l}\sigma_{\rho} \\
(")\end{array}$ & $\begin{array}{c}\theta \\
\left(^{\circ}\right)\end{array}$ & $\begin{array}{l}\sigma_{\theta} \\
\left(^{\circ}\right)\end{array}$ & $\Delta m$ & Notes & Orbit & $\begin{array}{r}\Delta \rho(\mathrm{O}-\mathrm{C}) \\
(")\end{array}$ & $\begin{array}{r}\Delta \theta(\mathrm{O}-\mathrm{C}) \\
\left({ }^{\circ}\right)\end{array}$ \\
\hline $07237+0953$ & HEI727 & 2012.156 & 2 & 1.075 & 0.019 & $72.5^{*}$ & 0.9 & & & & & \\
\hline $07238+1831$ & WSI4 & 2011.184 & 1 & 0.607 & 0.007 & 142.6 & 1.6 & & & & & \\
\hline $07239+2212$ & COU277 & 2012.142 & 1 & 0.952 & 0.007 & $247.5^{*}$ & 0.3 & & & & & \\
\hline $07239+3125$ & COU1243 & 2012.142 & 1 & 1.574 & 0.008 & $210.5^{*}$ & 0.3 & & & & & \\
\hline $07244+3041$ & COU1244 & 2011.184 & 1 & 1.052 & 0.044 & 93.9 & 1.4 & & & & & \\
\hline $07261+0747$ & A2944 & 2012.142 & 1 & 0.235 & 0.007 & $217.2^{*}$ & 0.6 & & & & & \\
\hline $07267+7144$ & MLR408 & 2012.202 & 1 & 1.529 & 0.008 & $260.3^{*}$ & 0.3 & & & & & \\
\hline $07268+3221$ & COU1413 & 2012.142 & 1 & 0.404 & 0.007 & $130.6^{*}$ & 1.0 & & & & & \\
\hline $07270+7901$ & MLR495 & 2012.202 & 1 & 0.940 & 0.007 & $347.5^{*}$ & 0.3 & & & & & \\
\hline $07292+1253$ & A 2868 & 2012.156 & 2 & 0.654 & 0.015 & $197.2^{*}$ & 0.3 & & & & & \\
\hline $07304+1352$ & STF1102AB & 2011.219 & 2 & 7.693 & 0.038 & 45.8 & 0.3 & & & & & \\
\hline $07305+0743$ & A2869 & 2012.238 & 1 & 0.173 & 0.007 & 119.1 & 2.1 & & & Msn2011c & -0.03 & -2.1 \\
\hline $07308+3033$ & COU1246 & 2011.219 & 2 & 1.715 & 0.032 & 7.4 & 1.1 & & & & & \\
\hline $07318+3151$ & COU1414 & 2011.219 & 2 & 1.978 & 0.022 & 8.0 & 0.9 & & & & & \\
\hline $07320+7520$ & A1069 & 2012.235 & 1 & 0.737 & 0.007 & $350.2^{*}$ & 0.3 & & & & & \\
\hline $07322+0703$ & TDS4903 & 2012.156 & 2 & - & - & - & - & & NR & & & \\
\hline $07322+1405$ & HU1244 & 2011.135 & 1 & 0.323 & 0.038 & 275.4 & 2.3 & & & Hrt2012a & -0.02 & 2.7 \\
\hline $07326+2405$ & COU89 & 2011.219 & 2 & 1.818 & 0.018 & 74.9 & 1.6 & & & & & \\
\hline $07336+1550$ & MCA32 & 2012.238 & 1 & 0.147 & 0.040 & 111.7 & 8.0 & & & Zir2008 & -0.05 & 4.3 \\
\hline $07343+1303$ & TDS4944 & 2012.156 & 2 & - & - & - & - & & NR & & & \\
\hline $07344+3307$ & BU579AB & 2011.228 & 1 & 0.861 & 0.017 & 35.1 & 1.1 & & & & & \\
\hline $07346+1156$ & A 2873 & 2011.228 & 1 & 1.719 & 0.011 & 113.8 & 0.3 & & & & & \\
\hline $07354+1950$ & COU475 & 2011.135 & 1 & 1.052 & 0.026 & 19.6 & 2.0 & & & & & \\
\hline $07358+3549$ & COU1887 & 2011.228 & 1 & 1.903 & 0.018 & 173.4 & 0.3 & & & & & \\
\hline $07370+2025$ & COU381 & 2011.184 & 1 & 0.381 & 0.007 & 121.4 & 0.5 & & & & & \\
\hline $07378+1522$ & HEI50 & 2011.178 & 1 & 0.786 & 0.020 & 172.3 & 1.7 & & & & & \\
\hline $07378+1522$ & HEI50 & 2012.156 & 2 & 0.756 & 0.041 & $168.9^{*}$ & 2.4 & & & & & \\
\hline $07379+7745$ & MLR478AB & 2012.235 & 1 & 1.914 & 0.010 & $32.2^{*}$ & 0.3 & & & & & \\
\hline $07383+5205$ & MLR671 & 2012.202 & 1 & 0.138 & 0.040 & 25.1 & 8.0 & & & & & \\
\hline $07387+1302$ & $\mathrm{~A} 2875 \mathrm{CD}$ & 2011.178 & 1 & 0.875 & 0.027 & 115.5 & 0.7 & & & & & \\
\hline $07387+1302$ & $\mathrm{~A} 2875 \mathrm{CD}$ & 2011.230 & 1 & 0.902 & 0.048 & 114.7 & 0.9 & & & & & \\
\hline $07393+0514$ & SHB1AB & 2012.241 & 1 & - & - & - & - & & NR & & & \\
\hline $07397+0117$ & A 2532 & 2012.238 & 1 & 0.542 & 0.007 & $278.5^{*}$ & 1.0 & & & & & \\
\hline $07398+0516$ & $\mathrm{BRD} 2 \mathrm{AB}$ & 2011.230 & 1 & 0.874 & 0.008 & 7.8 & 1.1 & & & & & \\
\hline $07414+8240$ & HU839 & 2012.235 & 1 & 0.215 & 0.007 & 118.7 & 1.3 & & & & & \\
\hline $07417+0942$ & STF1130 & 2011.184 & 1 & 0.473 & 0.012 & 50.7 & 2.4 & & & Msn1999a & 0.02 & -0.8 \\
\hline $07417+1803$ & A 2876 & 2011.135 & 1 & 0.438 & 0.026 & 51.4 & 0.9 & & & & & \\
\hline $07417+3726$ & STT177 & 2011.228 & 1 & 0.549 & 0.010 & 149.2 & 1.1 & & & Sca2018e & 0.01 & 0.5 \\
\hline $07418+6734$ & HDS1087Aa,Ab & 2012.202 & 1 & 0.356 & 0.007 & $346.6^{*}$ & 0.9 & & & & & \\
\hline $07420+1145$ & A 2877 & 2011.178 & 1 & 0.684 & 0.035 & 59.5 & 1.9 & & & & & \\
\hline $07420+1145$ & A 2877 & 2011.178 & 1 & 0.669 & 0.027 & 61.2 & 1.1 & & & & & \\
\hline $07421+0217$ & HEI733 & 2011.230 & 1 & 0.811 & 0.015 & 93.0 & 0.7 & & & & & \\
\hline $07421+0217$ & HEI733 & 2011.230 & 1 & 0.821 & 0.014 & 93.7 & 0.5 & & & & & \\
\hline $07423+0319$ & HEI734 & 2012.238 & 1 & 1.291 & 0.007 & 143.0 & 0.3 & & & & & \\
\hline $07425+1315$ & HU1245 & 2011.230 & 1 & 1.731 & 0.010 & 82.5 & 0.3 & & & & & \\
\hline $07429+6517$ & HU843 & 2012.202 & 1 & 0.249 & 0.014 & $61.9^{*}$ & 2.4 & & & & & \\
\hline $07449+6112$ & MLR43 & 2012.202 & 1 & 0.204 & 0.027 & $233.2^{*}$ & 3.2 & & & & & \\
\hline $07449+6112$ & MLR43 & 2012.235 & 1 & 0.172 & 0.030 & 232.1 & 8.0 & & & & & \\
\hline $07452+7548$ & STF1107 & 2012.230 & 1 & 1.214 & 0.007 & $215.1^{*}$ & 0.3 & & & & & \\
\hline $07453+2802$ & BU580CD & 2011.228 & 1 & 1.337 & 0.049 & 131.8 & 2.9 & & & & & \\
\hline $07454+0251$ & $\mathrm{~A} 2878 \mathrm{AC}$ & 2011.230 & 1 & 1.227 & 0.026 & 114.1 & 0.3 & & & & & \\
\hline $07461+2107$ & HO247 & 2012.244 & 1 & 0.482 & 0.011 & $257.4^{*}$ & 0.5 & & & USN1999b & -0.03 & 0.4 \\
\hline
\end{tabular}




\begin{tabular}{|c|c|c|c|c|c|c|c|c|c|c|c|c|}
\hline WDS & Name & Epoch & Bin. & $\begin{array}{c}\rho \\
(")\end{array}$ & $\begin{array}{l}\sigma_{\rho} \\
(")\end{array}$ & $\begin{array}{c}\theta \\
\left({ }^{\circ}\right)\end{array}$ & $\begin{array}{l}\sigma_{\theta} \\
\left(^{\circ}\right)\end{array}$ & $\Delta m$ & Notes & Orbit & $\begin{array}{r}\Delta \rho(\mathrm{O}-\mathrm{C}) \\
(")\end{array}$ & $\begin{array}{r}\Delta \theta(\mathrm{O}-\mathrm{C}) \\
\left({ }^{\circ}\right)\end{array}$ \\
\hline $07466+0151$ & A2879 & 2011.230 & 1 & 1.265 & 0.027 & 151.5 & 1.3 & 3.43 & & & & \\
\hline $07466+0151$ & A2879 & 2011.230 & 1 & 1.220 & 0.024 & 155.3 & 3.3 & & & & & \\
\hline $07476+2528$ & HO36 & 2012.246 & 1 & 0.607 & 0.007 & $125.9^{*}$ & 0.9 & & & & & \\
\hline $07480+6018$ & HU1247 & 2012.202 & 1 & 0.168 & 0.040 & 44.9 & 8.0 & & & Sod1999 & -0.01 & $-3.5^{Q}$ \\
\hline $07480+6018$ & HU1247 & 2012.202 & 1 & 0.167 & 0.040 & 45.1 & 8.0 & & & Sod1999 & -0.01 & $-3.3^{Q}$ \\
\hline $07484+5310$ & A1330 & 2012.230 & 1 & 0.512 & 0.007 & $117.2^{*}$ & 0.7 & & & & & \\
\hline $07486+2308$ & WRH15 & 2011.135 & 1 & 0.173 & 0.044 & 22.8 & 8.0 & & & USN2002 & -0.09 & -8.4 \\
\hline $07486+2308$ & WRH15 & 2012.238 & 1 & 0.234 & 0.027 & $30.5^{*}$ & 2.3 & & & USN2002 & -0.03 & 0.1 \\
\hline $07486+2308$ & BU1062AB,C & 2012.238 & 1 & 4.337 & 0.025 & 33.4 & 0.3 & & & & & \\
\hline $07505+1205$ & HEI342 & 2011.219 & 2 & 0.921 & 0.018 & 144.4 & 2.7 & & & & & \\
\hline $07506+0650$ & A 2743 & 2012.156 & 2 & 0.595 & 0.015 & $204.1^{*}$ & 1.3 & & & & & \\
\hline $07513+3849$ & STF1145 & 2011.219 & 2 & 1.349 & 0.015 & 41.5 & 1.2 & & & & & \\
\hline $07519+1820$ & COU43 & 2012.156 & 2 & 1.097 & 0.208 & $144.1^{*}$ & 6.1 & & & & & \\
\hline $07519+2215$ & COU927 & 2012.142 & 1 & 0.942 & 0.011 & $209.7^{*}$ & 0.3 & & & & & \\
\hline $07527+6247$ & MLR135 & 2012.202 & 1 & 0.534 & 0.007 & $281.2^{*}$ & 0.3 & & & & & \\
\hline $07532+0811$ & A2952 & 2012.142 & 1 & 0.342 & 0.007 & $63.1^{*}$ & 0.8 & & & & & \\
\hline $07536+1531$ & HEI54 & 2011.184 & 1 & 0.494 & 0.009 & 120.0 & 2.4 & & & & & \\
\hline $07545+6008$ & HDS1123 & 2012.230 & 1 & 0.755 & 0.007 & $175.0^{*}$ & 0.6 & & & & & \\
\hline $07550+1023$ & A2953 & 2011.230 & 1 & 1.911 & 0.021 & 161.6 & 0.3 & & & & & \\
\hline $07550+1023$ & A2953 & 2012.156 & 2 & 2.062 & 0.015 & $162.6^{*}$ & 0.3 & & & & & \\
\hline $07550+1023$ & A2953 & 2012.241 & 1 & 1.901 & 0.010 & $162.0^{*}$ & 0.3 & & & & & \\
\hline $07555+2422$ & A 2537 & 2012.241 & 1 & 0.182 & 0.040 & 33.0 & 8.0 & & & Zir2012b & -0.03 & -5.0 \\
\hline $07556+5831$ & A1072 & 2012.230 & 1 & 0.159 & 0.040 & 13.9 & 8.0 & & & & & \\
\hline $07558+2420$ & A 2538 & 2012.241 & 1 & 0.721 & 0.007 & $307.3^{*}$ & 0.3 & & & & & \\
\hline $07560+2342$ & COU929 & 2012.238 & 1 & 0.226 & 0.015 & $21.3^{*}$ & 1.4 & & & Hrt2009 & -0.03 & -2.5 \\
\hline $07560+2342$ & COU929 & 2012.142 & 1 & 0.215 & 0.011 & $22.8^{*}$ & 2.2 & 0.30 & & Hrt2009 & -0.04 & -0.8 \\
\hline $07560+2742$ & HDS1129 & 2011.184 & 1 & 0.325 & 0.022 & 43.1 & 3.8 & & & & & \\
\hline $07560+2742$ & HDS1129 & 2011.184 & 1 & 0.375 & 0.021 & 48.0 & 2.0 & & & & & \\
\hline $07563+1052$ & A2882 & 2012.142 & 1 & 2.029 & 0.010 & $214.7^{*}$ & 0.3 & & & & & \\
\hline $07563+5345$ & HDS1130 & 2012.235 & 1 & 0.198 & 0.040 & $242.3^{*}$ & 8.0 & & & & & \\
\hline $07564+0335$ & HEI736 & 2011.230 & 1 & 0.840 & 0.019 & 74.9 & 2.1 & 0.59 & & & & \\
\hline $07564+0335$ & HEI736 & 2011.230 & 1 & 0.854 & 0.017 & 77.0 & 2.0 & & & & & \\
\hline $07566+1954$ & COU930 & 2012.156 & 1 & 0.492 & 0.038 & $148.2^{*}$ & 1.4 & & & & & \\
\hline $07566+6634$ & HU846 & 2012.235 & 1 & 0.419 & 0.007 & $214.8^{*}$ & 1.5 & & & & & \\
\hline $07567+0721$ & HDS1132 & 2012.238 & 1 & 0.927 & 0.007 & $264.1^{*}$ & 0.5 & & & & & \\
\hline $07571+2058$ & HO250 & 2012.246 & 1 & 0.776 & 0.027 & $146.7^{*}$ & 0.3 & & & & & \\
\hline $07571+2058$ & $\mathrm{HO} 250$ & 2011.178 & 1 & 0.819 & 0.032 & 147.7 & 0.4 & & & & & \\
\hline $07583+1321$ & HEI136 & 2011.178 & 1 & 1.310 & 0.007 & 84.9 & 0.3 & & & & & \\
\hline $07583+2009$ & HU847 & 2011.228 & 1 & 0.544 & 0.021 & 29.1 & 1.0 & & & & & \\
\hline $07590+3528$ & COU1741 & 2011.219 & 2 & 1.008 & 0.027 & 96.3 & 0.7 & & & & & \\
\hline $07593+3817$ & COU2076 & 2011.228 & 1 & 0.500 & 0.007 & 96.9 & 0.7 & & & & & \\
\hline $07594+1021$ & HEI737 & 2011.228 & 1 & 1.353 & 0.007 & 103.7 & 0.3 & & & & & \\
\hline $07594+1021$ & HEI737 & 2011.228 & 1 & 1.356 & 0.007 & 103.4 & 1.6 & & & & & \\
\hline $08005+2900$ & OSO20 & 2012.241 & 1 & - & - & - & - & & NR & & & \\
\hline $08015+1402$ & HEI138 & 2011.184 & 1 & 0.745 & 0.021 & 74.5 & 1.1 & & & & & \\
\hline $08015+1402$ & HEI138 & 2011.178 & 1 & 0.748 & 0.023 & 75.0 & 0.9 & & & & & \\
\hline $08021+5437$ & STF1165AB & 2012.235 & 1 & 0.225 & 0.012 & $318.2^{*}$ & 1.6 & & & & & \\
\hline $08025+6305$ & HDS1142 & 2012.230 & 1 & 0.188 & 0.040 & 17.6 & 8.0 & & & & & \\
\hline $08026+2706$ & COU1113 & 2012.142 & 1 & 0.878 & 0.007 & $293.4^{*}$ & 0.3 & & & & & \\
\hline $08030+1843$ & COU477 & 2011.184 & 1 & 0.858 & 0.007 & 140.6 & 0.8 & & & & & \\
\hline $08031+6138$ & HDS1146 & 2012.235 & 1 & 0.696 & 0.021 & 96.6 & 0.3 & & & & & \\
\hline $08033+2153$ & COU163 & 2011.184 & 1 & 0.542 & 0.012 & 47.1 & 0.4 & & & & & \\
\hline $08034+2151$ & COU164 & 2011.184 & 1 & 0.165 & 0.040 & 41.1 & 8.0 & & & & & \\
\hline $08041+3302$ & STT187 & 2012.238 & 1 & 0.386 & 0.007 & 340.7 & 0.6 & & & Msn1999a & -0.03 & 1.7 \\
\hline
\end{tabular}




\begin{tabular}{|c|c|c|c|c|c|c|c|c|c|c|c|c|}
\hline WDS & Name & Epoch & Bin. & $\begin{array}{c}\rho \\
(")\end{array}$ & $\begin{array}{l}\sigma_{\rho} \\
(")\end{array}$ & $\begin{array}{c}\theta \\
\left({ }^{\circ}\right)\end{array}$ & $\begin{array}{l}\sigma_{\theta} \\
\left(^{\circ}\right)\end{array}$ & $\Delta m$ & Notes & Orbit & $\begin{array}{r}\Delta \rho(\mathrm{O}-\mathrm{C}) \\
(")\end{array}$ & $\begin{array}{r}\Delta \theta(\mathrm{O}-\mathrm{C}) \\
\left({ }^{\circ}\right)\end{array}$ \\
\hline $08044+1217$ & BU581AB & 2012.238 & 1 & 0.355 & 0.007 & $185.5^{*}$ & 0.4 & & & Hrt2000b & 0.00 & 2.1 \\
\hline $08044+1217$ & BU581AB,C & 2012.238 & 1 & 5.184 & 0.026 & 41.4 & 0.3 & & & & & \\
\hline $08044+3538$ & COU1889 & 2011.219 & 2 & 0.902 & 0.021 & 28.8 & 3.7 & & & & & \\
\hline $08050+5825$ & A1073 & 2012.230 & 1 & 0.337 & 0.007 & $135.9^{*}$ & 0.7 & & & Msn2017a & -0.02 & 0.2 \\
\hline $08057+1516$ & HEI56 & 2012.244 & 1 & 1.529 & 0.008 & $241.3^{*}$ & 0.3 & & & & & \\
\hline $08062+3213$ & COU1555 & 2011.219 & 1 & 0.181 & 0.040 & 84.3 & 8.0 & & & & & \\
\hline $08067+7334$ & HDS1153 & 2012.230 & 1 & - & - & - & - & & NR & & & \\
\hline $08070+0853$ & A 2957 & 2012.244 & 1 & 0.573 & 0.007 & $64.2^{*}$ & 0.6 & & & & & \\
\hline $08070+3538$ & COU1890 & 2011.219 & 1 & 0.880 & 0.007 & 12.0 & 0.6 & & & & & \\
\hline $08071+5407$ & A1333 & 2012.235 & 1 & 0.441 & 0.010 & $24.9^{*}$ & 0.3 & & & & & \\
\hline $08073+1213$ & HEI473 & 2012.156 & 2 & 1.864 & 0.026 & $211.4^{*}$ & 1.3 & & & & & \\
\hline $08076+3432$ & COU1556 & 2012.142 & 1 & 0.908 & 0.007 & $327.0^{*}$ & 0.5 & & & & & \\
\hline $08081+5627$ & HDS1154Aa,Ab & 2012.235 & 1 & 0.107 & 0.040 & 118.0 & 8.0 & & & & & \\
\hline $08081+5627$ & HDS1154Aa,Ab & 2012.235 & 1 & 0.175 & 0.040 & 116.6 & 8.0 & & & & & \\
\hline $08095+5339$ & MLR698 & 2012.230 & 1 & 0.955 & 0.007 & $32.5^{*}$ & 0.3 & & & & & \\
\hline $08108+3443$ & HDS1164 & 2011.228 & 1 & 0.364 & 0.024 & 199.8 & 2.6 & & & & & \\
\hline $08108+3443$ & HDS1164 & 2012.244 & 1 & 0.303 & 0.019 & $197.9^{*}$ & 0.6 & & & & & \\
\hline $08113+7422$ & A1074 & 2012.235 & 1 & 0.937 & 0.007 & $33.3^{*}$ & 0.3 & & & & & \\
\hline $08115+2209$ & COU278 & 2011.230 & 1 & 1.836 & 0.024 & 135.3 & 0.8 & 1.51 & & & & \\
\hline $08115+2209$ & COU278 & 2011.230 & 1 & 1.813 & 0.009 & 135.9 & 1.1 & & & & & \\
\hline $08122+1739$ & STF1196AB & 2011.178 & 1 & 1.091 & 0.012 & 34.9 & 0.3 & & & Izm2019 & 0.02 & 0.5 \\
\hline $08126+2849$ & COU1114 & 2012.244 & 1 & 0.101 & 0.040 & 15.8 & 8.0 & & & & & \\
\hline $08130+1547$ & A 2544 & 2011.228 & 1 & 1.327 & 0.007 & 129.0 & 0.3 & & & & & \\
\hline $08136+1023$ & BU204 & 2012.244 & 1 & 0.749 & 0.007 & $288.8^{*}$ & 0.8 & & & & & \\
\hline $08142+1741$ & BU1243AB & 2011.228 & 1 & 1.688 & 0.010 & 149.8 & 0.3 & & & & & \\
\hline $08152+1550$ & HEI140 & 2011.228 & 1 & 0.354 & 0.027 & 2.2 & 0.4 & & & & & \\
\hline $08155+0738$ & A2958 & 2011.184 & 1 & 0.410 & 0.018 & 293.8 & 1.6 & & & & & \\
\hline $08155+2124$ & COU279 & 2012.238 & 1 & 0.188 & 0.040 & 115.4 & 8.0 & & & & & \\
\hline $08160+1842$ & JCT3Aa,Ab & 2012.142 & 1 & - & - & - & - & & NR & & & \\
\hline $08160+1842$ & JCT3Aa,Ab & 2012.244 & 1 & - & - & - & - & & NR & & & \\
\hline $08160+1842$ & HO524AB & 2012.142 & 2 & 4.416 & 0.022 & 341.9 & 0.3 & & & & & \\
\hline $08160+1842$ & HO524AB & 2012.142 & 1 & 4.379 & 0.022 & $341.9^{*}$ & 0.3 & & & & & \\
\hline $08160+1842$ & HO524AB & 2012.244 & 1 & 4.383 & 0.022 & $342.0^{*}$ & 0.3 & & & & & \\
\hline $08161+7648$ & MLR496 & 2012.230 & 1 & 0.126 & 0.057 & $142.0^{*}$ & 7.7 & & & & & \\
\hline $08164+3326$ & COU1557 & 2011.184 & 1 & 0.391 & 0.007 & 87.0 & 1.1 & & & & & \\
\hline $08164+3326$ & COU1557 & 2011.178 & 1 & 0.378 & 0.007 & 87.2 & 1.5 & & & & & \\
\hline $08170+7158$ & MLR497 & 2012.230 & 1 & 0.805 & 0.007 & $301.6^{*}$ & 0.3 & & & & & \\
\hline $08176+1050$ & A2959 & 2011.230 & 1 & 0.802 & 0.009 & 87.4 & 0.6 & & & & & \\
\hline $08176+1050$ & A 2959 & 2011.230 & 1 & 0.777 & 0.018 & 85.0 & 0.7 & 1.38 & & & & \\
\hline $08190+7624$ & MLR498 & 2012.230 & 1 & 0.773 & 0.007 & $131.3^{*}$ & 0.4 & & & & & \\
\hline $08191+1810$ & COU952 & 2011.184 & 1 & 0.141 & 0.040 & 38.4 & 8.0 & & & & & \\
\hline $08191+1810$ & COU952 & 2012.142 & 1 & 0.114 & 0.040 & 27.7 & 8.0 & & & & & \\
\hline $08193+4052$ & A 2362 & 2011.252 & 1 & 0.590 & 0.007 & $171.5^{*}$ & 0.6 & & & & & \\
\hline $08199+2155$ & COU280 & 2012.244 & 1 & 1.587 & 0.010 & $155.4^{*}$ & 0.3 & & & & & \\
\hline $08201+7153$ & MLR499 & 2012.230 & 1 & 0.118 & 0.040 & 8.0 & 8.0 & & & & & \\
\hline $08201+7153$ & MLR499 & 2012.235 & 1 & - & - & - & - & & NR & & & \\
\hline $08204+0540$ & A2891 & 2011.230 & 1 & 1.052 & 0.085 & 40.7 & 6.7 & 0.13 & & & & \\
\hline $08204+0540$ & A2891 & 2011.230 & 1 & 1.118 & 0.027 & 40.7 & 0.6 & & & & & \\
\hline $08209+1149$ & HEI475 & 2011.228 & 1 & 1.181 & 0.038 & 69.5 & 0.8 & & & & & \\
\hline $08215+6947$ & MLR411 & 2012.235 & 1 & 0.837 & 0.007 & $229.2^{*}$ & 0.7 & & & & & \\
\hline
\end{tabular}




\begin{tabular}{|c|c|c|c|c|c|c|c|c|c|c|c|c|}
\hline WDS & Name & Epoch & Bin. & $\begin{array}{c}\rho \\
(")\end{array}$ & $\begin{array}{l}\sigma_{\rho} \\
(")\end{array}$ & $\begin{array}{c}\theta \\
\left({ }^{\circ}\right)\end{array}$ & $\begin{array}{l}\sigma_{\theta} \\
\left(^{\circ}\right)\end{array}$ & $\Delta m$ & Notes & Orbit & $\begin{array}{r}\Delta \rho(\mathrm{O}-\mathrm{C}) \\
(")\end{array}$ & $\begin{array}{r}\Delta \theta(\mathrm{O}-\mathrm{C}) \\
\left({ }^{\circ}\right)\end{array}$ \\
\hline $08220+5314$ & MLR699 & 2012.235 & 1 & 0.345 & 0.007 & $32.5^{*}$ & 0.4 & & & & & \\
\hline $08221+1551$ & HEI142 & 2011.178 & 1 & 0.662 & 0.015 & 24.8 & 0.9 & & & & & \\
\hline $08231+2001$ & $\mathrm{HO} 525 \mathrm{AB}$ & 2011.228 & 1 & 0.443 & 0.016 & 347.8 & 2.8 & & & FMR2013e & 0.03 & 2.2 \\
\hline $08244+6453$ & HU853 & 2012.230 & 1 & 0.390 & 0.007 & $168.8^{*}$ & 0.3 & & & & & \\
\hline $08247+4200$ & HDS1197 & 2011.258 & 1 & 0.296 & 0.025 & $83.0^{*}$ & 2.3 & & & & & \\
\hline $08251+1210$ & HEI477 & 2011.179 & 1 & 1.013 & 0.015 & 39.2 & 0.5 & & & & & \\
\hline $08254+7450$ & MLR479 & 2012.230 & 1 & 0.604 & 0.007 & $304.5^{*}$ & 0.8 & & & & & \\
\hline $08254+7607$ & MLR547 & 2012.230 & 1 & 1.332 & 0.007 & $227.8^{*}$ & 0.3 & & & & & \\
\hline $08262+3528$ & COU1891 & 2011.179 & 1 & 0.843 & 0.031 & 160.1 & 1.2 & & & & & \\
\hline $08264+1749$ & COU953 & 2011.184 & 1 & 0.863 & 0.007 & 33.1 & 1.5 & & & & & \\
\hline $08267+8136$ & HDS1202 & 2012.235 & 1 & 0.632 & 0.010 & 145.4 & 0.6 & & & & & \\
\hline $08268+1610$ & HEI143 & 2012.156 & 2 & 2.624 & 0.022 & $306.1^{*}$ & 0.5 & & & & & \\
\hline $08269+3212$ & $\mathrm{HU} 714 \mathrm{Ba}, \mathrm{Bb}$ & 2012.252 & 1 & 0.758 & 0.007 & $312.9^{*}$ & 0.3 & & & Msn2017c & 0.00 & 0.9 \\
\hline $08269+3212$ & $\mathrm{HU} 714 \mathrm{Ba}, \mathrm{Bb}$ & 2011.179 & 1 & 0.759 & 0.015 & 312.0 & 2.0 & & & Msn2017c & -0.00 & 1.6 \\
\hline $08273+3802$ & HDS1205 & 2011.219 & 1 & 0.741 & 0.011 & 87.1 & 2.9 & & & & & \\
\hline $08279+0313$ & HDS1206 & 2011.230 & 1 & 1.662 & 0.009 & 146.3 & 0.3 & & & & & \\
\hline $08286+3502$ & WOR19 & 2012.246 & 1 & 0.506 & 0.007 & $199.1^{*}$ & 0.3 & & & FMR2012j & -0.01 & -1.7 \\
\hline $08286+5439$ & MLR672 & 2012.235 & 1 & 0.125 & 0.040 & 106.7 & 8.0 & & & & & \\
\hline $08292+3852$ & COU2081 & 2011.252 & 1 & 1.926 & 0.027 & $29.4^{*}$ & 0.7 & & & & & \\
\hline $08292+3852$ & COU2081 & 2011.252 & 1 & 1.961 & 0.010 & 28.7 & 0.4 & & & & & \\
\hline $08297+7017$ & MLR413 & 2012.235 & 1 & 1.160 & 0.008 & $199.9^{*}$ & 0.3 & & & & & \\
\hline $08303+4022$ & COU2497 & 2011.258 & 1 & 0.548 & 0.015 & 77.7 & 0.7 & & & & & \\
\hline $08304+7134$ & MLR414 & 2012.235 & 1 & 0.667 & 0.007 & $323.6^{*}$ & 0.3 & & & & & \\
\hline $08310+4012$ & SKF207 & 2012.252 & 1 & - & - & - & - & & NR & & & \\
\hline $08313+1151$ & HDS1217 & 2012.244 & 1 & 0.241 & 0.007 & $272.2^{*}$ & 1.6 & & & & & \\
\hline $08330+0958$ & A2895AB & 2011.230 & 1 & 0.835 & 0.007 & 54.9 & 1.1 & 0.04 & & & & \\
\hline $08330+0958$ & A2895AB & 2011.230 & 1 & 0.841 & 0.007 & 55.6 & 0.4 & & & & & \\
\hline $08334+1319$ & HEI346 & 2011.228 & 1 & 0.765 & 0.007 & 187.2 & 0.3 & & & & & \\
\hline $08334+1319$ & HEI346 & 2012.156 & 2 & 0.754 & 0.024 & $185.7^{*}$ & 1.6 & & & & & \\
\hline $08345+6711$ & HDS1228 & 2012.230 & 1 & - & - & - & - & & NR & & & \\
\hline $08351+4349$ & COU2615 & 2011.252 & 1 & 0.151 & 0.040 & 125.2 & 8.0 & & & & & \\
\hline $08360+2050$ & BOV29 & 2012.241 & 1 & 0.295 & 0.014 & 118.5 & 1.1 & & & & & \\
\hline $08360+2050$ & BOV29 & 2012.244 & 2 & - & - & - & - & & NR & & & \\
\hline $08364+2441$ & SKF690 & 2012.244 & 2 & 1.734 & 0.015 & 355.5 & 0.3 & 2.12 & & & & \\
\hline $08364+2441$ & SKF690 & 2012.244 & 2 & 1.805 & 0.019 & $356.6^{*}$ & 2.2 & & & & & \\
\hline $08364+2441$ & SKF690 & 2012.244 & 2 & 1.703 & 0.035 & 355.3 & 2.3 & 2.45 & & & & \\
\hline $08372+2017$ & BOV31 & 2012.241 & 1 & - & - & - & - & & NR & & & \\
\hline $08375+1906$ & BOV32 & 2012.244 & 2 & - & - & - & - & & NR & & & \\
\hline $08376+1829$ & BOV33 & 2012.241 & 1 & - & - & - & - & & NR & & & \\
\hline $08382+1158$ & HU858 & 2011.230 & 1 & 0.722 & 0.020 & 152.4 & 0.3 & 0.81 & & & & \\
\hline $08382+1158$ & HU858 & 2011.230 & 1 & 0.759 & 0.033 & 152.9 & 1.0 & & & & & \\
\hline $08384+1256$ & HDS1243 & 2011.179 & 1 & 0.443 & 0.013 & 21.7 & 2.1 & & & & & \\
\hline $08384+2006$ & BOV36 & 2012.241 & 1 & - & - & - & - & & NR & & & \\
\hline $08395+7314$ & A1079 & 2012.230 & 1 & 0.485 & 0.010 & $183.3^{*}$ & 0.3 & & & & & \\
\hline $08396+1926$ & BOV39 & 2012.244 & 2 & 0.928 & 0.025 & 140.4 & 1.1 & & & & & \\
\hline $08396+1926$ & BOV39 & 2012.241 & 1 & - & - & - & - & & NR & & & \\
\hline $08401+5629$ & MLR673 & 2012.235 & 1 & 0.574 & 0.007 & $282.8^{*}$ & 0.3 & & & & & \\
\hline $08402+4105$ & A2128 & 2011.258 & 1 & 1.706 & 0.009 & $113.0^{*}$ & 0.3 & & & & & \\
\hline $08403+2012$ & BOV40 & 2012.246 & 1 & - & - & - & - & & NR & & & \\
\hline $08403+1947$ & BOV41 & 2012.238 & 1 & 0.235 & 0.007 & $247.1^{*}$ & 1.3 & & & & & \\
\hline $08403+4617$ & A1748 & 2011.252 & 1 & 0.762 & 0.011 & 143.4 & 1.2 & & & & & \\
\hline $08403+4617$ & A1748 & 2011.252 & 1 & 0.781 & 0.013 & 144.3 & 0.3 & & & & & \\
\hline $08403+4617$ & A1748 & 2011.252 & 1 & 0.762 & 0.007 & 144.3 & 0.3 & & & & & \\
\hline
\end{tabular}




\begin{tabular}{|c|c|c|c|c|c|c|c|c|c|c|c|c|}
\hline WDS & Name & Epoch & Bin. & $\begin{array}{c}\rho \\
(")\end{array}$ & $\begin{array}{l}\sigma_{\rho} \\
(")\end{array}$ & $\begin{array}{c}\theta \\
\left({ }^{\circ}\right)\end{array}$ & $\begin{array}{l}\sigma_{\theta} \\
\left(^{\circ}\right)\end{array}$ & $\Delta m$ & Notes & Orbit & $\begin{array}{r}\Delta \rho(\mathrm{O}-\mathrm{C}) \\
(")\end{array}$ & $\begin{array}{r}\Delta \theta(\mathrm{O}-\mathrm{C}) \\
\left({ }^{\circ}\right)\end{array}$ \\
\hline $08404+1940$ & $\mathrm{BOV} 42 \mathrm{Ba}, \mathrm{Bb}$ & 2012.241 & 1 & - & - & - & - & & $\mathrm{NR}$ & & & \\
\hline $08404+1913$ & BOV43 & 2012.241 & 1 & - & - & - & - & & NR & & & \\
\hline $08404+1913$ & BOV43 & 2012.241 & 1 & - & - & - & - & & NR & & & \\
\hline $08407+1839$ & BOV46 & 2012.246 & 2 & - & - & - & - & & NR & & & \\
\hline $08407+3449$ & HDS1249 & 2011.228 & 1 & 1.015 & 0.010 & 65.5 & 0.3 & & & & & \\
\hline $08407+3449$ & HDS1249 & 2011.219 & 1 & 1.021 & 0.013 & 63.3 & 1.3 & & & & & \\
\hline $08409+4226$ & HDS1251 & 2011.252 & 1 & 0.733 & 0.009 & $37.1^{*}$ & 0.8 & & & & & \\
\hline $08411+1904$ & BOV48 & 2012.241 & 1 & 0.360 & 0.007 & 120.0 & 0.3 & & & & & \\
\hline $08411+1904$ & BOV48 & 2012.241 & 1 & 0.383 & 0.007 & 117.8 & 0.3 & 0.65 & & & & \\
\hline $08411+4352$ & A1749 & 2012.246 & 1 & 0.587 & 0.009 & $107.8^{*}$ & * 1.2 & & & & & \\
\hline $08413+2029$ & BU585 & 2011.266 & 1 & 0.285 & 0.010 & $75.5^{*}$ & 1.3 & & & & & \\
\hline $08415+1852$ & BOV49 & 2012.241 & 1 & 1.850 & 0.009 & 230.5 & 0.3 & 4.46 & & & & \\
\hline $08417+4617$ & COU2616 & 2011.263 & 1 & 0.737 & 0.015 & 141.3 & 1.5 & & & & & \\
\hline $08425+2342$ & TDS6051 & 2011.266 & 1 & 1.578 & 0.008 & $333.8^{*}$ & ${ }^{*} 0.3$ & & & & & \\
\hline $08425+3656$ & COU2082 & 2011.219 & 1 & 0.492 & 0.015 & 26.2 & 1.0 & & & & & \\
\hline $08427+2603$ & HO354 & 2011.266 & 1 & 0.944 & 0.007 & $186.8^{*}$ & ${ }^{*} 0.3$ & & & & & \\
\hline $08430+2020$ & BOV51 & 2012.252 & 1 & - & - & - & - & & NR & & & \\
\hline $08432+3849$ & BU209 & 2011.263 & 1 & 1.271 & 0.007 & $8.6^{*}$ & 0.3 & & & & & \\
\hline $08435+3341$ & POP97 & 2011.228 & 1 & 0.959 & 0.018 & 96.4 & 0.5 & & & & & \\
\hline $08439+4403$ & A1752 & 2011.263 & 1 & 0.330 & 0.013 & $72.2^{*}$ & 0.6 & & & & & \\
\hline $08441+1357$ & HEI145BC & 2012.246 & 1 & 0.900 & 0.051 & $165.4^{*}$ & ${ }^{*} 0.4$ & & & & & \\
\hline $08444+1555$ & $\mathrm{~A} 2472$ & 2011.266 & 1 & 0.796 & 0.007 & 81.2 & 0.3 & & & & & \\
\hline $08445+4057$ & HDS1257 & 2011.252 & 1 & 0.210 & 0.016 & $212.5^{*}$ & ${ }^{*} 7.1$ & & & & & \\
\hline $08452+1941$ & BOV54 & 2012.238 & 1 & 0.428 & 0.007 & $232.8^{*}$ & * 0.6 & & & & & \\
\hline $08454+7039$ & HDS1263 & 2012.203 & 1 & 0.296 & 0.082 & 155.8 & 9.8 & & & & & \\
\hline $08456+2916$ & COU1250 & 2011.179 & 1 & 1.055 & 0.015 & 3.8 & 0.8 & & & & & \\
\hline $08456+8442$ & A1078 & 2012.235 & 1 & 0.497 & 0.007 & $142.7^{*}$ & ${ }^{*} 0.3$ & & & Lin2010c & -0.09 & -11.8 \\
\hline $08462+1811$ & HDS1268 & 2011.219 & 1 & 0.375 & 0.061 & 6.9 & 2.9 & & & & & \\
\hline $08462+1811$ & HDS1268 & 2011.228 & 1 & 0.312 & 0.018 & 9.7 & 2.2 & & & & & \\
\hline $08468+0625$ & SP1AB & 2012.238 & 1 & 0.244 & 0.009 & $172.8^{*}$ & ${ }^{*} 0.6$ & & & Sod1999 & -0.03 & -1.0 \\
\hline $08468+0625$ & $\mathrm{SP} 1 \mathrm{AB}, \mathrm{C}$ & 2012.238 & 1 & 2.722 & 0.014 & 124.0 & 0.3 & & & & & \\
\hline $08472+1110$ & STF1276 & 2011.263 & 1 & 1.249 & 0.007 & $88.5^{*}$ & 0.3 & & & & & \\
\hline $08488+5406$ & MLR674 & 2012.203 & 1 & 0.275 & 0.007 & $127.0^{*}$ & ${ }^{*} 0.4$ & & & & & \\
\hline $08491+2357$ & COU1118 & 2011.179 & 1 & 2.100 & 0.010 & 130.4 & 0.3 & & & & & \\
\hline $08491+2357$ & COU1118 & 2011.179 & 1 & 2.097 & 0.015 & 129.8 & 0.5 & & & & & \\
\hline $08495+0852$ & BU1068 & 2011.266 & 1 & 0.325 & 0.010 & $324.7^{*}$ & ${ }^{*} 0.5$ & & & Hrt2010a & -0.04 & 2.8 \\
\hline $08502+0647$ & HEI744 & 2011.266 & 1 & 0.336 & 0.075 & 174.4 & 1.0 & & & & & \\
\hline $08505+6741$ & HDS1280 & 2012.235 & 1 & - & - & - & - & & NR & & & \\
\hline $08507+1800$ & A 2473 & 2011.179 & 1 & 0.215 & 0.014 & 84.1 & 1.3 & & & Doc2016d & -0.03 & -0.9 \\
\hline $08510+3124$ & HO40 & 2011.220 & 1 & 0.885 & 0.063 & 96.1 & 1.5 & & & & & \\
\hline $08526+4212$ & COU2618 & 2012.246 & 1 & 0.347 & 0.010 & $163.5^{*}$ & ${ }^{*} 0.8$ & & & & & \\
\hline $08526+4212$ & COU2618 & 2011.252 & 1 & 0.379 & 0.007 & $338.1^{*}$ & ${ }^{*} 1.5$ & & & & & \\
\hline $08527+4437$ & COU2617 & 2012.252 & 1 & 0.762 & 0.007 & $226.9^{*}$ & ${ }^{*} 0.3$ & & & & & \\
\hline $08531+5457$ & A1584 & 2012.235 & 1 & 0.675 & 0.007 & $87.3^{*}$ & 0.3 & & & Msn2014a & 0.01 & -0.9 \\
\hline $08536+1210$ & HEI480 & 2011.272 & 1 & 1.555 & 0.008 & $90.1^{*}$ & 0.5 & & & & & \\
\hline $08536+1210$ & HEI480 & 2011.272 & 1 & 1.570 & 0.008 & $93.6^{*}$ & 0.3 & & & & & \\
\hline $08536+1210$ & HEI480 & 2012.238 & 1 & 1.535 & 0.008 & 92.8 & 0.3 & & & & & \\
\hline $08539+1958$ & COU773 & 2011.179 & 1 & - & - & - & - & & NR & & & \\
\hline $08542+1935$ & HDS1286 & 2011.228 & 1 & 0.871 & 0.016 & 171.8 & 2.9 & & & & & \\
\hline $08542+3035$ & STF1291 & 2011.263 & 1 & 1.513 & 0.008 & $310.5^{*}$ & ${ }^{*} 0.3$ & & & Izm2019 & -0.01 & 0.1 \\
\hline $08544+3647$ & ES2105 & 2011.263 & 1 & 1.784 & 0.009 & $112.7^{*}$ & ${ }^{*} 0.3$ & & & & & \\
\hline $08546+6211$ & MLR140 & 2012.203 & 1 & - & - & - & - & & $\mathrm{NR}$ & & & \\
\hline $08547+4919$ & HDS1287 & 2011.252 & 1 & 1.694 & 0.017 & $202.3^{*}$ & ${ }^{*} 0.5$ & & & & & \\
\hline $08547+4919$ & HDS1287 & 2011.252 & 1 & 1.703 & 0.013 & 202.6 & 0.3 & & & & & \\
\hline
\end{tabular}




\begin{tabular}{|c|c|c|c|c|c|c|c|c|c|c|c|c|}
\hline WDS & Name & Epoch & Bin. & $\begin{array}{c}\rho \\
(")\end{array}$ & $\begin{array}{l}\sigma_{\rho} \\
(")\end{array}$ & $\begin{array}{c}\theta \\
\left({ }^{\circ}\right)\end{array}$ & $\begin{array}{l}\sigma_{\theta} \\
\left({ }^{\circ}\right)\end{array}$ & $\Delta m$ & Notes & Orbit & $\begin{array}{r}\Delta \rho(\mathrm{O}-\mathrm{C}) \\
(")\end{array}$ & $\begin{array}{r}\Delta \theta(\mathrm{O}-\mathrm{C}) \\
\left({ }^{\circ}\right)\end{array}$ \\
\hline $08547+4919$ & HDS1287 & 2011.252 & 1 & 1.683 & 0.008 & $201.5^{*}$ & 0.3 & & & & & \\
\hline $08548+1446$ & HDS1288 & 2011.228 & 1 & 0.275 & 0.052 & 54.8 & 3.6 & & & & & \\
\hline $08561+4341$ & STF3120 & 2011.258 & 1 & 1.365 & 0.007 & $181.6^{*}$ & 0.3 & & & & & \\
\hline $08563+4745$ & HDS1292 & 2011.252 & 1 & 0.169 & 0.040 & 140.1 & 13.2 & & & & & \\
\hline $08567+0130$ & GII39AC & 2011.269 & 1 & 1.806 & 0.012 & 54.9 & 0.3 & & $\mathrm{ND}$ & & & \\
\hline $08567+0130$ & HEI745AC & 2012.000 & 1 & 1.030 & 0.015 & 174.4 & 0.5 & & $\mathrm{ND}$ & & & \\
\hline $08568+4228$ & A2133 & 2011.263 & 1 & 1.331 & 0.007 & $179.7^{*}$ & 0.3 & & & & & \\
\hline $08579+6419$ & HDS1298 & 2012.203 & 1 & 0.134 & 0.040 & 169.3 & 8.0 & & & & & \\
\hline $08580+3014$ & $\mathrm{HO} 252$ & 2011.179 & 1 & 0.112 & 0.040 & 37.5 & 8.0 & & & & & \\
\hline $08580+3726$ & HU860 & 2011.258 & 1 & 0.852 & 0.013 & 6.3 & 0.6 & & & & & \\
\hline $08581+2634$ & A1973 & 2012.246 & 1 & 1.167 & 0.007 & $102.2^{*}$ & 0.3 & & & & & \\
\hline $08585+3548$ & COU1897 & 2011.252 & 1 & 0.182 & 0.040 & 268.8 & 8.0 & & & Doc2013b & 0.09 & -7.4 \\
\hline $08585+3707$ & COU2083 & 2012.246 & 1 & 0.776 & 0.007 & $306.1^{*}$ & 0.6 & & & & & \\
\hline $08588+1414$ & HU861 & 2011.179 & 1 & 0.170 & 0.040 & 24.6 & 8.0 & & & & & \\
\hline $08588+8117$ & HDS1300 & 2012.235 & 1 & 0.156 & 0.040 & 16.4 & 8.0 & & & & & \\
\hline $08589+4607$ & COU2685 & 2011.252 & 1 & 1.160 & 0.018 & $200.7^{*}$ & 1.0 & & & & & \\
\hline $08593+3457$ & STF1296 & 2011.263 & 1 & 1.768 & 0.009 & $75.2^{*}$ & 0.4 & & & & & \\
\hline $09002+1351$ & HU862 & 2011.135 & 1 & 0.529 & 0.024 & 53.6 & 3.2 & & & & & \\
\hline $09007+3208$ & COU1253 & 2011.179 & 1 & 0.754 & 0.024 & 8.3 & 0.9 & & & & & \\
\hline $09017+3943$ & HDS1311 & 2011.252 & 1 & 0.533 & 0.014 & $39.8^{*}$ & 1.0 & & & & & \\
\hline $09018+0321$ & HEI746 & 2011.266 & 1 & 0.321 & 0.014 & $260.8^{*}$ & 4.4 & & & & & \\
\hline $09027+0252$ & HEI747 & 2011.266 & 1 & 0.875 & 0.025 & 301.6 & 1.3 & & & & & \\
\hline $09027+0252$ & HEI747 & 2011.266 & 1 & 0.895 & 0.010 & $303.3^{*}$ & 0.8 & & & & & \\
\hline $09037+4531$ & COU2686 & 2011.263 & 1 & 0.439 & 0.007 & $98.9^{*}$ & 0.6 & & & & & \\
\hline $09042+0547$ & A2971 & 2011.266 & 1 & 0.294 & 0.007 & 158.8 & 0.5 & & & & & \\
\hline $09043+4008$ & COU2498 & 2011.258 & 1 & - & - & - & - & & NR & & & \\
\hline $09044+1451$ & HEI57 & 2011.272 & 1 & 1.776 & 0.009 & 131.7 & 0.3 & & & & & \\
\hline $09044+7024$ & MLR415 & 2012.230 & 1 & 1.221 & 0.008 & $127.8^{*}$ & 0.3 & & & & & \\
\hline $09048+6455$ & MLR322 & 2012.203 & 1 & 1.132 & 0.007 & $157.1^{*}$ & 0.3 & & & & & \\
\hline $09050+6216$ & MLR141 & 2012.203 & 1 & 0.745 & 0.007 & $284.8^{*}$ & 0.4 & & & & & \\
\hline $09050+7702$ & HDS1317 & 2012.203 & 1 & 1.027 & 0.007 & $273.5^{*}$ & 0.3 & & & & & \\
\hline $09058+3607$ & HU865 & 2011.228 & 1 & 0.459 & 0.015 & 170.6 & 1.3 & & & & & \\
\hline $09058+6807$ & MLR700 & 2012.203 & 1 & 0.176 & 0.040 & $194.3^{*}$ & 8.0 & & & & & \\
\hline $09062+3314$ & GII37Ba,Bb & 2011.179 & 1 & 0.340 & 0.007 & 88.6 & 0.3 & 0.06 & $\mathrm{ND}$ & & & \\
\hline $09062+3314$ & GII37Ba,Bb & 2011.179 & 1 & 0.342 & 0.007 & 88.7 & 0.3 & 0.06 & $\mathrm{ND}$ & & & \\
\hline $09062+3314$ & GII37Ba,Bb & 2011.184 & 1 & 0.338 & 0.007 & 89.6 & 0.3 & 0.10 & $\mathrm{ND}$ & & & \\
\hline $09062+3314$ & GII37Ba,Bb & 2011.184 & 1 & 0.331 & 0.007 & 90.1 & 0.3 & 0.10 & ND & & & \\
\hline $09062+3314$ & GII37Ba,Bb & 2012.252 & 1 & 0.323 & 0.007 & $94.4^{*}$ & 0.3 & 0.03 & $\mathrm{ND}$ & & & \\
\hline $09062+3314$ & COU1560AB & 2011.179 & 1 & 1.393 & 0.010 & 114.3 & 0.5 & & & & & \\
\hline $09062+3314$ & COU1560AB & 2011.179 & 1 & 1.384 & 0.007 & 114.3 & 0.8 & & & & & \\
\hline $09062+3314$ & COU1560AB & 2011.184 & 1 & 1.378 & 0.007 & 115.7 & 0.3 & & & & & \\
\hline $09064+6302$ & HU123 & 2012.230 & 1 & 0.761 & 0.009 & $233.2^{*}$ & 0.3 & & & & & \\
\hline $09068+4707$ & COU2687 & 2011.258 & 2 & - & - & - & - & & NR & & & \\
\hline $09073+2211$ & COU165 & 2011.135 & 1 & 1.565 & 0.015 & 172.4 & 0.4 & & & & & \\
\hline $09083+4211$ & A1976 & 2011.263 & 1 & 0.378 & 0.007 & $259.5^{*}$ & 0.8 & & & & & \\
\hline $09088+4416$ & COU2619 & 2011.253 & 1 & 1.154 & 0.010 & $240.3^{*}$ & 0.3 & & & & & \\
\hline $09094+2203$ & WRH16AC & 2011.135 & 1 & 2.167 & 0.013 & 8.2 & 0.4 & & & & & ND \\
\hline $09094+2203$ & WRH16AB & 2012.238 & 1 & - & - & - & - & & NR & & & \\
\hline
\end{tabular}




\begin{tabular}{|c|c|c|c|c|c|c|c|c|c|c|c|c|}
\hline WDS & Name & Epoch & Bin. & $\begin{array}{c}\rho \\
(")\end{array}$ & $\begin{array}{l}\sigma_{\rho} \\
(")\end{array}$ & $\begin{array}{c}\theta \\
\left({ }^{\circ}\right)\end{array}$ & $\begin{array}{l}\sigma_{\theta} \\
\left(^{\circ}\right)\end{array}$ & $\Delta m$ & Notes & Orbit & $\begin{array}{r}\Delta \rho(\mathrm{O}-\mathrm{C}) \\
(")\end{array}$ & $\begin{array}{r}\Delta \theta(\mathrm{O}-\mathrm{C}) \\
\left({ }^{\circ}\right)\end{array}$ \\
\hline $09095+3250$ & COU1561 & 2012.244 & 1 & 0.742 & 0.007 & $212.0^{*}$ & * 0.6 & & & & & \\
\hline $09095+3250$ & COU1561 & 2011.179 & 1 & 0.753 & 0.015 & 211.3 & 1.3 & & & & & \\
\hline $09100+4034$ & COU2499 & 2012.252 & 1 & 0.612 & 0.007 & $218.3^{*}$ & ${ }^{*} 1.1$ & & & & & \\
\hline $09123+1500$ & FIN347Aa, Ab & 2011.135 & 1 & 0.118 & 0.040 & 141.8 & 8.0 & & & Msn2018b & -0.04 & 0.6 \\
\hline $09124+2653$ & A1977 & 2011.228 & 1 & 0.272 & 0.007 & 147.5 & 3.6 & & & & & \\
\hline $09126+0915$ & HEI483 & 2011.266 & 1 & 0.888 & 0.007 & $226.8^{*}$ & ${ }^{*} 0.3$ & & & & & \\
\hline $09128+6943$ & MLR416 & 2012.203 & 1 & 0.499 & 0.007 & $68.9^{*}$ & 0.5 & & & & & \\
\hline $09129+1040$ & A 2974 & 2011.230 & 1 & 1.785 & 0.009 & 59.9 & 0.3 & & & & & \\
\hline $09134+3131$ & COU1562 & 2012.252 & 1 & 2.029 & 0.013 & $293.4^{*}$ & ${ }^{*} 0.3$ & & & & & \\
\hline $09138+4443$ & HDS1335 & 2011.263 & 1 & 0.249 & 0.014 & 89.8 & 2.0 & & & & & \\
\hline $09138+4443$ & HDS1335 & 2011.263 & 1 & 0.316 & 0.008 & 10.2 & 0.7 & & & & & \\
\hline $09143+0438$ & HEI349 & 2011.266 & 1 & 1.598 & 0.008 & $51.3^{*}$ & 0.6 & & & & & \\
\hline $09143+5911$ & MLR701 & 2012.203 & 1 & 1.435 & 0.007 & $21.9^{*}$ & 0.3 & & & & & \\
\hline $09144+1118$ & HEI484 & 2011.266 & 1 & 0.201 & 0.013 & 176.5 & 1.9 & & & & & \\
\hline $09146+8253$ & HU864 & 2012.230 & 1 & 0.717 & 0.007 & $32.2^{*}$ & 0.3 & & & & & \\
\hline $09149+0427$ & HEI350 & 2011.230 & 1 & 0.722 & 0.017 & 44.1 & 0.3 & & & Tok2016e & -0.03 & -2.2 \\
\hline $09153+0602$ & A2754 & 2011.176 & 1 & 1.142 & 0.009 & 168.8 & 1.0 & & & & & \\
\hline $09154+2248$ & A2136 & 2011.179 & 1 & 1.863 & 0.010 & 113.4 & 0.3 & & & & & \\
\hline $09159+2431$ & COU934Aa,Ab & 2012.246 & 1 & 0.251 & 0.017 & 175.4 & 0.6 & & & & & \\
\hline $09159+2431$ & A1980 & 2011.228 & 1 & 1.593 & 0.057 & 51.4 & 0.8 & & & & & \\
\hline $09159+2431$ & A1980 & 2012.246 & 1 & 1.568 & 0.012 & 53.1 & 0.3 & & & & & \\
\hline $09168+4040$ & COU19AB & 2012.246 & 2 & 2.400 & 0.015 & 346.7 & 0.3 & 0.19 & & & & \\
\hline $09168+4040$ & COU19AB & 2012.246 & 2 & 2.400 & 0.015 & $348.4^{*}$ & ${ }^{*} 0.4$ & & & & & \\
\hline $09168+4040$ & COU19BC & 2012.246 & 2 & 7.908 & 0.070 & 38.6 & 1.3 & & & & & \\
\hline $09172+4038$ & A1982 & 2012.246 & 1 & 0.231 & 0.011 & $336.7^{*}$ & ${ }^{*} 6.8$ & & & & & \\
\hline $09172+4038$ & A1982 & 2011.253 & 1 & 0.263 & 0.007 & $339.7^{*}$ & ${ }^{*} 2.0$ & & & & & \\
\hline $09175+7715$ & KUI39 & 2012.203 & 1 & 0.545 & 0.008 & $91.2^{*}$ & 0.5 & & & Mnt1999a & 0.09 & 8.7 \\
\hline $09181+0245$ & A 2556 & 2011.230 & 1 & 0.928 & 0.015 & 169.8 & 0.8 & 1.31 & & & & \\
\hline $09181+0245$ & A 2556 & 2011.230 & 1 & 0.950 & 0.007 & 169.6 & 1.0 & & & & & \\
\hline $09181+0245$ & A 2556 & 2012.000 & 1 & 0.948 & 0.012 & 170.2 & 2.0 & & & & & \\
\hline $09183+1847$ & COU384 & 2012.246 & 1 & 0.185 & 0.040 & $55.9^{*}$ & 8.0 & & & & & \\
\hline $09185+1547$ & HEI150 & 2011.228 & 1 & 0.336 & 0.069 & 80.4 & 0.8 & & & & & \\
\hline $09186+2944$ & $\mathrm{~A} 221 \mathrm{Ba}, \mathrm{Bb}$ & 2012.238 & 1 & 0.205 & 0.010 & $231.1^{*}$ & * 4.4 & & & Tok2019e & 0.04 & 15.7 \\
\hline $09188+4011$ & COU2384 & 2011.253 & 1 & 0.871 & 0.007 & $133.2^{*}$ & ${ }^{*} 0.6$ & & & & & \\
\hline $09190+1057$ & A 2975 & 2011.176 & 1 & 1.352 & 0.015 & 3.7 & 0.9 & & & & & \\
\hline $09201+7644$ & HDS1344 & 2012.203 & 1 & - & - & - & - & & NR & & & \\
\hline $09207+0810$ & A2976BC & 2011.266 & 1 & 1.031 & 0.008 & $137.4^{*}$ & ${ }^{*} 0.4$ & & & & & \\
\hline $09215+1943$ & $\mathrm{~A} 127$ & 2011.266 & 1 & 1.452 & 0.007 & $32.9^{*}$ & 0.3 & & & & & \\
\hline $09218+8834$ & COU10 & 2012.230 & 1 & 1.752 & 0.009 & 77.3 & 0.3 & & & & & \\
\hline $09219+3313$ & POP106 & 2011.258 & 2 & 1.523 & 0.041 & 66.6 & 0.3 & & & & & \\
\hline $09222+3239$ & HDS1347 & 2011.228 & 1 & 1.197 & 0.016 & 16.8 & 0.7 & & & & & \\
\hline $09226+0725$ & A 2977 & 2011.176 & 1 & 1.635 & 0.029 & 87.0 & 0.3 & & & & & \\
\hline $09227+7020$ & TDS529 & 2012.235 & 1 & 0.800 & 0.010 & $342.9^{*}$ & ${ }^{*} 0.3$ & & & & & \\
\hline $09233+0921$ & HEI487 & 2011.230 & 1 & 2.266 & 0.044 & 104.6 & 0.8 & 0.68 & & & & \\
\hline $09237+4441$ & A1759 & 2011.253 & 1 & 1.659 & 0.008 & 139.4 & 0.3 & & & & & \\
\hline $09242+1219$ & HEI488 & 2011.266 & 1 & 1.339 & 0.007 & $183.9^{*}$ & ${ }^{*} 0.3$ & & & & & \\
\hline $09245+1808$ & A 2477 & 2011.176 & 1 & 0.414 & 0.028 & 0.5 & 1.0 & & & FMR2014a & -0.02 & 0.0 \\
\hline $09247+2641$ & HDS1351 & 2011.179 & 1 & 0.220 & 0.028 & 15.2 & 0.9 & & & & & \\
\hline $09249+5134$ & STT200 & 2011.258 & 2 & 1.153 & 0.015 & 157.3 & 0.6 & & & & & \\
\hline $09251+2616$ & BU1070 & 2011.228 & 1 & 0.365 & 0.010 & 89.7 & 1.1 & & & & & \\
\hline $09252+1449$ & HU869 & 2011.266 & 1 & 0.752 & 0.007 & 93.5 & 0.3 & & & & & \\
\hline $09252+4606$ & HDS1353 & 2011.263 & 1 & 0.405 & 0.007 & $161.2^{*}$ & ${ }^{*} 0.4$ & & & Cve2016b & 0.00 & 1.1 \\
\hline $09260+1854$ & COU935 & 2011.135 & 1 & 0.872 & 0.021 & 60.4 & 2.3 & & & & & \\
\hline
\end{tabular}




\begin{tabular}{|c|c|c|c|c|c|c|c|c|c|c|c|c|}
\hline WDS & Name & Epoch & Bin. & $\begin{array}{c}\rho \\
(")\end{array}$ & $\begin{array}{l}\sigma_{\rho} \\
(")\end{array}$ & $\begin{array}{c}\theta \\
\left(^{\circ}\right)\end{array}$ & $\begin{array}{l}\sigma_{\theta} \\
\left(^{\circ}\right)\end{array}$ & $\Delta m$ & Notes & Orbit & $\begin{array}{r}\Delta \rho(\mathrm{O}-\mathrm{C}) \\
(")\end{array}$ & $\begin{array}{r}\Delta \theta(\mathrm{O}-\mathrm{C}) \\
\left({ }^{\circ}\right)\end{array}$ \\
\hline $09260+1854$ & COU935 & 2011.135 & 1 & 0.866 & 0.040 & 59.8 & 1.2 & & & & & \\
\hline $09260+2839$ & A222 & 2012.246 & 1 & 0.391 & 0.007 & $10.9^{*}$ & 1.6 & & & Hrt2014b & -0.00 & 2.4 \\
\hline $09261+1258$ & HEI353 & 2011.135 & 1 & 0.614 & 0.021 & 141.7 & 1.3 & & & & & \\
\hline $09261+5344$ & A1343 & 2012.235 & 1 & 0.262 & 0.008 & $55.9^{*}$ & 0.7 & & & & & \\
\hline $09267+3855$ & COU1898 & 2011.220 & 1 & 1.650 & 0.015 & 164.2 & 0.8 & & & & & \\
\hline $09268+0558$ & $\mathrm{~A} 2755$ & 2011.231 & 1 & 2.094 & 0.014 & 122.1 & 0.3 & & & & & \\
\hline $09276+0858$ & HEI489 & 2011.231 & 1 & 0.610 & 0.007 & 5.3 & 1.7 & & & & & \\
\hline $09276+0858$ & HEI489 & 2011.231 & 1 & 0.593 & 0.040 & 3.6 & 1.6 & 0.37 & & & & \\
\hline $09277+4456$ & A1762 & 2011.253 & 1 & 0.791 & 0.013 & 107.2 & 0.6 & & & & & \\
\hline $09277+4456$ & A1762 & 2011.258 & 2 & 0.778 & 0.021 & 105.9 & 0.6 & & & & & \\
\hline $09277+4456$ & A1762 & 2011.263 & 1 & 0.807 & 0.007 & $106.3^{*}$ & 0.3 & & & & & \\
\hline $09278+2250$ & A129 & 2011.179 & 1 & 1.571 & 0.022 & 4.0 & 1.1 & & & & & \\
\hline $09279+3128$ & HO366AB & 2012.252 & 1 & 0.224 & 0.040 & $212.9^{*}$ & 6.0 & & & Zir2010 & 0.07 & -49.0 \\
\hline $09282+2811$ & COU1121 & 2011.179 & 1 & 0.950 & 0.007 & 35.2 & 0.6 & & & & & \\
\hline $09284+3955$ & TDS6583 & 2011.263 & 1 & - & - & - & - & & NR & & & \\
\hline $09285+1212$ & HU871 & 2011.231 & 1 & 1.376 & 0.007 & 138.5 & 0.3 & & & & & \\
\hline $09285+1212$ & HU871 & 2011.231 & 1 & 1.348 & 0.036 & 138.0 & 0.5 & 0.89 & & & & \\
\hline $09290+1917$ & COU936 & 2011.179 & 1 & 0.944 & 0.018 & 46.1 & 0.8 & & & & & \\
\hline $09292+4526$ & TDS536 & 2011.258 & 2 & - & - & - & - & & NR & & & \\
\hline $09299+5808$ & MLR549AB & 2012.203 & 1 & 0.207 & 0.007 & $138.0^{*}$ & 2.5 & & & & & \\
\hline $09299+5808$ & MLR549AC & 2012.203 & 1 & 2.647 & 0.013 & $178.7^{*}$ & 0.3 & & & & & \\
\hline $09300+4216$ & A1985 & 2011.258 & 2 & 1.593 & 0.015 & $24.1^{*}$ & 0.7 & & & & & \\
\hline $09305+2026$ & A130 & 2012.197 & 1 & 0.984 & 0.018 & $280.8^{*}$ & 1.1 & & & & & \\
\hline $09307+4604$ & A1764 & 2011.253 & 1 & 0.142 & 0.040 & 105.7 & 8.0 & & & & & \\
\hline $09307+6532$ & HDS1366 & 2012.235 & 1 & 0.166 & 0.040 & $76.7^{*}$ & 8.0 & & & & & \\
\hline $09317+8512$ & A1081 & 2012.230 & 1 & 0.165 & 0.057 & $268.9^{*}$ & 1.7 & & & & & \\
\hline $09321+2347$ & HDS1372 & 2011.220 & 1 & 0.579 & 0.027 & 50.0 & 1.8 & & & & & \\
\hline $09321+2347$ & HDS1372 & 2011.220 & 1 & 0.579 & 0.023 & 48.7 & 0.7 & & & & & \\
\hline $09321+2347$ & HDS1372 & 2011.228 & 1 & 0.577 & 0.008 & 50.8 & 0.6 & & & & & \\
\hline $09326+3744$ & COU1899 & 2011.220 & 1 & 1.350 & 0.021 & 155.5 & 0.4 & & & & & \\
\hline $09327+1549$ & HEI152 & 2011.179 & 1 & 0.364 & 0.034 & 170.4 & 1.0 & & & & & \\
\hline $09327+1549$ & HEI152 & 2011.228 & 1 & 0.364 & 0.021 & 171.2 & 0.3 & & & & & \\
\hline $09333+0910$ & A 2756 & 2011.231 & 1 & 1.433 & 0.044 & 178.6 & 1.0 & 0.34 & & & & \\
\hline $09336+6216$ & HU228 & 2012.203 & 1 & 0.588 & 0.007 & $72.5^{*}$ & 0.3 & & & & & \\
\hline $09338+3231$ & COU1415 & 2012.244 & 1 & 0.173 & 0.040 & 116.0 & 8.0 & & & & & \\
\hline $09338+3634$ & HU1127 & 2011.220 & 1 & 0.380 & 0.020 & 31.3 & 1.4 & & & & & \\
\hline $09338+7302$ & HDS1377 & 2012.235 & 1 & 0.156 & 0.040 & 181.8 & 8.0 & & & & & \\
\hline $09344+4704$ & COU2500 & 2012.252 & 1 & 0.928 & 0.007 & $231.6^{*}$ & 0.3 & & & & & \\
\hline $09346+4123$ & HDS1381 & 2011.253 & 1 & 1.034 & 0.007 & $146.7^{*}$ & 0.6 & & & & & \\
\hline $09346+4818$ & HDS1380 & 2011.263 & 1 & 0.830 & 0.010 & $299.4^{*}$ & 0.5 & & & & & \\
\hline $09347+1627$ & A2478AB & 2011.266 & 1 & 1.165 & 0.007 & $40.2^{*}$ & 0.4 & & & & & \\
\hline $09349+0515$ & $\mathrm{~A} 2757$ & 2011.231 & 1 & 0.305 & 0.050 & 52.9 & 7.5 & 1.10 & & Lin2017b & -0.02 & -5.8 \\
\hline $09349+0515$ & $\mathrm{~A} 2757$ & 2011.231 & 1 & 0.328 & 0.018 & 53.8 & 3.6 & & & Lin2017b & 0.01 & -4.9 \\
\hline $09349+2152$ & COU282 & 2011.266 & 1 & 1.151 & 0.007 & $120.8^{*}$ & 0.3 & & & & & \\
\hline $09354+4719$ & COU2501 & 2011.258 & 2 & 1.802 & 0.015 & $216.7^{*}$ & 0.3 & & & & & \\
\hline $09357+1836$ & COU385 & 2011.266 & 1 & 0.742 & 0.007 & $152.1^{*}$ & 0.3 & & & & & \\
\hline $09357+5004$ & HU565 & 2011.258 & 2 & 1.960 & 0.015 & $4.2^{*}$ & 0.3 & & & & & \\
\hline $09366+0503$ & A 2758 & 2011.184 & 1 & 0.166 & 0.040 & 13.7 & 8.0 & & & & & \\
\hline $09375+2838$ & HDS1385 & 2012.244 & 1 & 1.370 & 0.027 & $90.0^{*}$ & 0.6 & & & & & \\
\hline $09376+1528$ & A 2479 & 2012.197 & 1 & 0.311 & 0.007 & $247.2^{*}$ & 0.3 & & & Hei1978a & 0.04 & 2.9 \\
\hline $09376+1528$ & A 2479 & 2012.197 & 1 & 0.277 & 0.009 & $245.1^{*}$ & 0.5 & & & Hei1978a & 0.01 & 0.8 \\
\hline $09378+4800$ & HDS1386 & 2011.253 & 1 & 0.793 & 0.007 & $357.2^{*}$ & 0.6 & & & & & \\
\hline $09379+4554$ & A1765 & 2012.252 & 1 & 0.161 & 0.040 & 3.0 & 8.0 & & & Doc2012a & 0.03 & -1.9 \\
\hline
\end{tabular}




\begin{tabular}{|c|c|c|c|c|c|c|c|c|c|c|c|c|}
\hline WDS & Name & Epoch & Bin. & $\begin{array}{c}\rho \\
(")\end{array}$ & $\begin{array}{l}\sigma_{\rho} \\
(")\end{array}$ & $\begin{array}{c}\theta \\
\left({ }^{\circ}\right)\end{array}$ & $\begin{array}{l}\sigma_{\theta} \\
\left(^{\circ}\right)\end{array}$ & $\Delta m$ & Notes & Orbit & $\begin{array}{r}\Delta \rho(\mathrm{O}-\mathrm{C}) \\
(")\end{array}$ & $\begin{array}{r}\Delta \theta(\mathrm{O}-\mathrm{C}) \\
\left({ }^{\circ}\right)\end{array}$ \\
\hline $09379+7421$ & HDS1387 & 2012.203 & 1 & 0.165 & 0.040 & 169.3 & 8.0 & & & & & \\
\hline $09380+7610$ & HDS1388 & 2012.230 & 1 & 0.629 & 0.007 & $131.7^{*}$ & 0.7 & & & & & \\
\hline $09387+2132$ & HDS1389AC & 2011.176 & 1 & 0.401 & 0.015 & 88.8 & 1.4 & & & & & \\
\hline $09387+2132$ & HDS1389AB & 2011.176 & 1 & 0.134 & 0.040 & 25.2 & 8.0 & & & & & \\
\hline $09387+7516$ & MLR419 & 2012.203 & 1 & 0.387 & 0.007 & $334.7^{*}$ & 0.3 & & & & & \\
\hline $09388+4748$ & TDS6715 & 2011.263 & 1 & - & - & - & - & & $\mathrm{NR}$ & & & \\
\hline $09390+3017$ & COU1254 & 2012.252 & 1 & 0.557 & 0.007 & $87.9^{*}$ & 0.3 & & & & & \\
\hline $09391+1425$ & HDS1391 & 2011.176 & 1 & 0.858 & 0.011 & 136.8 & 0.9 & & & & & \\
\hline $09411+1756$ & $\mathrm{~A} 2480$ & 2011.184 & 1 & 1.163 & 0.007 & 157.1 & 1.7 & & & & & \\
\hline $09413+0256$ & HEI752 & 2011.176 & 1 & 1.002 & 0.007 & 156.6 & 0.7 & & & & & \\
\hline $09415+1753$ & A2481 & 2011.220 & 1 & 0.324 & 0.007 & 164.2 & 1.5 & & & & & \\
\hline $09421+3416$ & COU1566 & 2012.244 & 1 & 0.459 & 0.007 & 70.4 & 0.3 & & & & & \\
\hline $09421+3915$ & HU1252 & 2012.246 & 1 & 0.530 & 0.012 & $173.8^{*}$ & 1.1 & & & & & \\
\hline $09422+1332$ & HDS1397 & 2011.228 & 1 & 0.487 & 0.009 & 89.0 & 0.7 & & & & & \\
\hline $09423+5328$ & CHR29 & 2012.203 & 1 & 0.173 & 0.040 & 12.5 & 8.0 & & & & & \\
\hline $09426+1958$ & COU283 & 2011.135 & 1 & 0.597 & 0.018 & 52.3 & 4.7 & & & & & \\
\hline $09427+4826$ & HDS1399 & 2011.253 & 1 & 0.437 & 0.015 & $191.4^{*}$ & 1.2 & & & & & \\
\hline $09427+4826$ & HDS1399 & 2011.253 & 1 & 0.444 & 0.041 & $198.0^{*}$ & 2.8 & & & & & \\
\hline $09427+4826$ & HDS1399 & 2011.263 & 1 & 0.416 & 0.007 & $196.3^{*}$ & 0.5 & & & & & \\
\hline $09427+6016$ & HU229 & 2012.230 & 1 & 0.977 & 0.007 & $181.5^{*}$ & 0.5 & & & & & \\
\hline $09432+6709$ & MLR323 & 2012.203 & 1 & 0.367 & 0.007 & $24.3^{*}$ & 1.0 & & & & & \\
\hline $09437+3229$ & COU1567 & 2012.244 & 1 & 0.959 & 0.007 & $260.3^{*}$ & 0.3 & & & & & \\
\hline $09437+7202$ & MLR420 & 2012.203 & 1 & 0.173 & 0.040 & 116.8 & 8.0 & & & & & \\
\hline $09444+4429$ & HDS1405 & 2011.263 & 1 & 1.211 & 0.018 & 82.2 & 0.5 & & & & & \\
\hline $09450+5641$ & MLR675 & 2012.203 & 1 & 0.337 & 0.009 & $100.4^{*}$ & 0.3 & & & & & \\
\hline $09452+4109$ & COU2085 & 2011.253 & 1 & 0.128 & 0.040 & 163.4 & 12.4 & & & & & \\
\hline $09452+4109$ & SKF478AB & 2012.246 & 1 & 1.844 & 0.009 & 135.6 & 0.3 & & & & & \\
\hline $09452+4109$ & SKF478AB & 2012.246 & 1 & 1.844 & 0.009 & 135.0 & 0.3 & & & & & \\
\hline $09458+2500$ & COU776 & 2011.220 & 1 & 1.007 & 0.015 & 65.2 & 1.2 & 0.09 & & & & \\
\hline $09458+2500$ & COU776 & 2011.220 & 1 & 1.061 & 0.027 & 64.5 & 0.9 & & & & & \\
\hline $09473+7908$ & HDS1415 & 2012.230 & 1 & 0.121 & 0.040 & 174.3 & 8.0 & & & & & \\
\hline $09476+1419$ & MRL2 & 2011.184 & 1 & 0.442 & 0.029 & 101.9 & 2.4 & & & & & \\
\hline $09477+1023$ & HEI490 & 2011.135 & 1 & 0.242 & 0.016 & 60.7 & 1.7 & & & & & \\
\hline $09477+2036$ & COU284 & 2012.197 & 1 & - & - & - & - & & NR & & & \\
\hline $09478+1004$ & HO253 & 2011.266 & 1 & 1.248 & 0.007 & $298.6^{*}$ & 0.3 & & & & & \\
\hline $09487+5217$ & MLR676 & 2012.203 & 1 & 0.263 & 0.007 & $207.9^{*}$ & 0.4 & & & & & \\
\hline $09494+1832$ & HDS1419 & 2011.228 & 1 & 0.154 & 0.040 & 25.7 & 8.1 & & & & & \\
\hline $09495+3910$ & TDS6832 & 2011.263 & 1 & - & - & - & - & & NR & & & \\
\hline $09498+5254$ & MLR677 & 2012.230 & 1 & 0.162 & 0.040 & 132.8 & 8.0 & & & & & \\
\hline $09499+3036$ & COU1256 & 2011.220 & 1 & 1.179 & 0.034 & 148.5 & 1.1 & 0.08 & & & & \\
\hline $09499+3036$ & COU1256 & 2011.220 & 1 & 1.235 & 0.022 & 150.6 & 0.5 & & & & & \\
\hline $09499+3036$ & COU1256 & 2011.228 & 1 & 1.211 & 0.023 & 150.6 & 1.3 & & & & & \\
\hline $09500+1658$ & COU937 & 2011.135 & 1 & 1.489 & 0.015 & 116.9 & 0.7 & & & & & \\
\hline $09506+3331$ & COU1568 & 2012.920 & 1 & 0.970 & 0.007 & $31.3^{*}$ & 0.3 & & & & & \\
\hline $09512+3629$ & НО369 & 2012.246 & 1 & 0.311 & 0.007 & $105.2^{*}$ & 0.5 & & & Alz2020c & -0.04 & -0.2 \\
\hline $09512+7654$ & MLR525 & 2012.230 & 1 & 1.736 & 0.009 & $13.1^{*}$ & 0.3 & & & & & \\
\hline $09517+2617$ & COU1122 & 2011.266 & 1 & 0.964 & 0.007 & $267.4^{*}$ & 0.3 & & & & & \\
\hline $09519+4112$ & TDS6864 & 2011.263 & 1 & - & - & - & - & & $\mathrm{NR}$ & & & \\
\hline $09521+2916$ & A344 & 2011.220 & 1 & 0.628 & 0.017 & 69.7 & 1.5 & & & & & \\
\hline $09521+2916$ & A344 & 2011.220 & 1 & 0.633 & 0.017 & 68.7 & 1.3 & 1.05 & & & & \\
\hline $09522+0807$ & A2762 & 2011.135 & 1 & 0.480 & 0.021 & 76.2 & 2.4 & & & Tok2019c & 0.01 & 0.1 \\
\hline $09523+4058$ & A2139 & 2011.258 & 2 & 1.963 & 0.015 & $292.8^{*}$ & 0.5 & & & & & \\
\hline $09526+6143$ & HDS1426 & 2012.230 & 1 & 1.742 & 0.009 & $42.8^{*}$ & 0.3 & & & & & \\
\hline $09530+2638$ & COU1123 & 2012.197 & 1 & 0.449 & 0.007 & $75.8^{*}$ & 0.4 & & & & & \\
\hline
\end{tabular}




\begin{tabular}{|c|c|c|c|c|c|c|c|c|c|c|c|c|}
\hline WDS & Name & Epoch & Bin. & $\begin{array}{c}\rho \\
(")\end{array}$ & $\begin{array}{l}\sigma_{\rho} \\
(")\end{array}$ & $\begin{array}{c}\theta \\
\left({ }^{\circ}\right)\end{array}$ & $\begin{array}{l}\sigma_{\theta} \\
\left(^{\circ}\right)\end{array}$ & $\Delta m$ & Notes & Orbit & $\begin{array}{r}\Delta \rho(\mathrm{O}-\mathrm{C}) \\
(")\end{array}$ & $\begin{array}{r}\Delta \theta(\mathrm{O}-\mathrm{C}) \\
\left({ }^{\circ}\right)\end{array}$ \\
\hline $09531+3920$ & A 2140 & 2011.258 & 2 & 0.986 & 0.025 & $64.9^{*}$ & 2.1 & & & & & \\
\hline $09531+3920$ & A2140 & 2011.263 & 1 & 0.952 & 0.007 & $65.6^{*}$ & 0.3 & & & & & \\
\hline $09533+3017$ & COU1257 & 2011.179 & 1 & 0.339 & 0.008 & 154.7 & 0.9 & & & & & \\
\hline $09533+3017$ & COU1257 & 2011.179 & 1 & 0.339 & 0.008 & 154.7 & 0.9 & & & & & \\
\hline $09535+1657$ & CHR219 & 2011.176 & 1 & 0.181 & 0.040 & 78.1 & 8.0 & & & Hrt2012a & -0.05 & 0.6 \\
\hline $09541+4408$ & TDS6890 & 2011.263 & 1 & - & - & - & - & & NR & & & \\
\hline $09542+2612$ & COU1124 & 2012.197 & 1 & 0.563 & 0.007 & $294.5^{*}$ & 0.5 & & & & & \\
\hline $09544+3041$ & COU1258 & 2012.246 & 1 & 0.192 & 0.040 & $227.9^{*}$ & 8.0 & & & Hor 2020 & 0.04 & $-1.2^{Q}$ \\
\hline $09548+6515$ & HDS1430 & 2012.230 & 1 & 0.346 & 0.007 & $191.8^{*}$ & 0.8 & & & & & \\
\hline $09561+2433$ & TDS6906 & 2011.184 & 1 & 0.985 & 0.013 & 66.0 & 0.3 & & & & & \\
\hline $09566+4359$ & POP151 & 2011.263 & 1 & 0.483 & 0.012 & $263.5^{*}$ & 0.5 & & & & & \\
\hline $09569+1946$ & COU388 & 2011.266 & 2 & 1.669 & 0.015 & $241.3^{*}$ & 1.1 & & & & & \\
\hline $09577+2540$ & COU938 & 2011.228 & 1 & 0.139 & 0.040 & 59.3 & 8.0 & & & & & \\
\hline $09581+3856$ & COU2086 & 2012.246 & 1 & - & - & - & - & & NR & & & \\
\hline $09584+4045$ & HDS1437 & 2011.253 & 1 & 0.232 & 0.009 & $237.4^{*}$ & 1.3 & & & & & \\
\hline $09584+4045$ & HDS1437 & 2011.253 & 1 & 0.208 & 0.018 & $237.0^{*}$ & 2.1 & & & & & \\
\hline $09587+3853$ & COU2087 & 2011.176 & 1 & 0.243 & 0.028 & 133.5 & 2.6 & & & & & \\
\hline $09587+3853$ & COU2087 & 2011.228 & 1 & 0.304 & 0.027 & 129.6 & 0.6 & & & & & \\
\hline $09591+5316$ & A1346 & 2012.236 & 1 & 0.559 & 0.007 & $180.5^{*}$ & 0.6 & & & Hrt2009 & -0.00 & 0.2 \\
\hline $09598+3128$ & HDS1442 & 2011.184 & 1 & 0.399 & 0.007 & 130.8 & 0.7 & & & & & \\
\hline $09598+3306$ & HDS1441 & 2011.176 & 1 & 0.511 & 0.010 & 100.7 & 1.5 & & & & & \\
\hline $09599+1610$ & A2482 & 2011.220 & 1 & 0.755 & 0.007 & 38.4 & 2.6 & 1.47 & & & & \\
\hline $09599+1610$ & A2482 & 2011.220 & 1 & 0.761 & 0.013 & 39.7 & 0.6 & & & & & \\
\hline $10000+2433$ & CHR145 & 2011.184 & 1 & 0.196 & 0.040 & 185.3 & 8.0 & & & Hrt2012a & -0.07 & -1.5 \\
\hline $10000+2433$ & CHR145 & 2011.179 & 1 & 0.237 & 0.024 & 185.8 & 1.2 & & & Hrt2012a & -0.03 & -1.0 \\
\hline $10007+4904$ & HDS1445 & 2011.253 & 1 & 0.199 & 0.040 & 182.1 & 8.0 & & & & & \\
\hline \multirow[t]{2}{*}{$10012+2502$} & SHN19 & 2012.241 & 1 & 0.348 & 0.013 & $274.8^{*}$ & 3.0 & & & & & \\
\hline & Bks2005 & 0.13 & 30.7 & & & & & & & & & \\
\hline $10017+1725$ & RUC7 & 2012.197 & 1 & - & - & - & - & & $\mathrm{NR}$ & & & \\
\hline $10018+4328$ & POP115 & 2011.258 & 2 & 1.999 & 0.015 & $90.2^{*}$ & 0.3 & & & & & \\
\hline $10020+5150$ & HDS1447 & 2011.263 & 1 & 0.157 & 0.058 & 21.6 & 0.9 & & & & & \\
\hline $10021+4722$ & HDS1448 & 2011.264 & 1 & 0.283 & 0.013 & $32.5^{*}$ & 3.7 & & & & & \\
\hline $10022+5323$ & MLR678 & 2012.203 & 1 & - & - & - & - & & $\mathrm{NR}$ & & & \\
\hline $10026+4622$ & STT210 & 2011.258 & 2 & 1.300 & 0.015 & $257.8^{*}$ & 0.3 & & & & & \\
\hline $10028+4953$ & HDS1450 & 2011.258 & 2 & 0.838 & 0.019 & 98.4 & 0.8 & & & & & \\
\hline $10040+2521$ & COU589 & 2012.200 & 1 & 0.414 & 0.007 & $67.7^{*}$ & 0.4 & & & & & \\
\hline $10040+3239$ & HU631 & 2012.241 & 1 & 0.815 & 0.007 & $256.4^{*}$ & 0.3 & & & & & \\
\hline $10056+3105$ & STF1406 & 2012.279 & 1 & 0.777 & 0.007 & $218.9^{*}$ & 0.5 & & & & & \\
\hline $10057+4103$ & A 2142 & 2011.258 & 1 & 1.049 & 0.011 & $295.6^{*}$ & 0.3 & & & & & \\
\hline $10057+7048$ & MLR421 & 2012.230 & 1 & 1.927 & 0.010 & $191.5^{*}$ & 0.3 & & & & & \\
\hline $10059+3412$ & COU1569 & 2011.253 & 1 & 0.165 & 0.040 & 75.1 & 8.0 & & & Doc2006i & 0.05 & -15.2 \\
\hline $10062+0911$ & HEI492 & 2011.231 & 1 & 1.219 & 0.014 & 92.5 & 0.6 & & & & & \\
\hline $10062+0911$ & HEI492 & 2011.231 & 1 & 1.219 & 0.019 & 92.9 & 0.3 & 0.78 & & & & \\
\hline $10063+3457$ & TDS7020 & 2011.264 & 1 & - & - & - & - & & NR & & & \\
\hline $10063+7418$ & MLR422 & 2012.203 & 1 & 0.308 & 0.007 & $91.3^{*}$ & 1.3 & & & & & \\
\hline $10067+1754$ & HDS1457 & 2011.179 & 1 & 0.412 & 0.007 & 65.6 & 1.9 & & & Tok2019c & -0.01 & -1.5 \\
\hline
\end{tabular}




\begin{tabular}{|c|c|c|c|c|c|c|c|c|c|c|c|c|}
\hline WDS & Name & Epoch & Bin. & $\begin{array}{c}\rho \\
(")\end{array}$ & $\begin{array}{l}\sigma_{\rho} \\
(")\end{array}$ & $\begin{array}{c}\theta \\
\left({ }^{\circ}\right)\end{array}$ & $\begin{array}{l}\sigma_{\theta} \\
\left({ }^{\circ}\right)\end{array}$ & $\Delta m$ & Notes & Orbit & $\begin{array}{r}\Delta \rho(\mathrm{O}-\mathrm{C}) \\
(")\end{array}$ & $\begin{array}{r}\Delta \theta(\mathrm{O}-\mathrm{C}) \\
\left({ }^{\circ}\right)\end{array}$ \\
\hline $10067+1754$ & HDS1457 & 2011.179 & 1 & 0.404 & 0.007 & 67.6 & 1.1 & & & Tok2019c & -0.02 & 0.5 \\
\hline $10067+1754$ & HDS1457 & 2011.184 & 1 & 0.415 & 0.014 & 66.2 & 1.5 & & & Tok2019c & -0.00 & -0.9 \\
\hline $10067+1754$ & HDS1457 & 2012.197 & 1 & 0.439 & 0.007 & $64.7^{*}$ & 1.2 & & & Tok2019c & 0.02 & -0.3 \\
\hline $10071+4504$ & HDS1459 & 2011.253 & 1 & 0.821 & 0.007 & $301.1^{*}$ & 0.5 & & & & & \\
\hline $10076+2207$ & COU167 & 2012.241 & 1 & 1.776 & 0.029 & 118.4 & 0.8 & & & & & \\
\hline $10083+0802$ & A 2564 & 2011.231 & 1 & 0.788 & 0.015 & 103.9 & 0.8 & & & & & \\
\hline $10083+0802$ & A 2564 & 2011.231 & 1 & 0.773 & 0.010 & 104.4 & 0.4 & 0.62 & & & & \\
\hline $10083+3136$ & KUI48 & 2011.135 & 1 & 0.127 & 0.040 & 163.3 & 8.0 & & & Hrt1996a & 0.00 & -1.1 \\
\hline $10083+3136$ & KUI48 & 2012.246 & 1 & 0.182 & 0.040 & 165.2 & 8.0 & & & Hrt1996a & 0.01 & -1.4 \\
\hline $10092+2147$ & COU168 & 2011.266 & 1 & 0.950 & 0.007 & 53.2 & 0.3 & & & & & \\
\hline $10093+2020$ & A 2145 & 2011.176 & 1 & 0.238 & 0.055 & 234.2 & 8.0 & & & Msn2011e & 0.07 & -9.6 \\
\hline $10093+2020$ & A 2145 & 2012.200 & 1 & 0.226 & 0.040 & $230.5^{*}$ & 8.0 & & & Msn2011e & 0.05 & -11.8 \\
\hline $10109+3628$ & HDS1466 & 2011.184 & 1 & 0.603 & 0.018 & 179.8 & 3.0 & & & & & \\
\hline $10110+1834$ & COU287 & 2011.220 & 1 & 1.401 & 0.027 & 93.7 & 0.7 & & & & & \\
\hline $10110+1834$ & COU287 & 2011.220 & 1 & 1.387 & 0.025 & 92.5 & 0.6 & 0.01 & & & & \\
\hline $10110+4040$ & HDS1467 & 2012.246 & 1 & - & - & - & - & & NR & & & \\
\hline $10110+4040$ & HDS1467 & 2011.253 & 1 & 0.248 & 0.052 & 172.0 & 8.9 & & & & & \\
\hline $10110+7508$ & KUI47 & 2012.203 & 1 & 1.871 & 0.009 & $121.2^{*}$ & 0.3 & & & Hei1994a & 0.05 & -2.5 \\
\hline $10116+1321$ & HU874 & 2011.228 & 1 & 0.173 & 0.040 & 284.3 & 9.1 & & & Doc2020d & 0.03 & 0.4 \\
\hline $10116+1321$ & HU874 & 2012.197 & 1 & 0.247 & 0.007 & 285.0 & 1.3 & & & Doc2020d & -0.04 & 0.3 \\
\hline $10118+0317$ & HEI756 & 2011.176 & 1 & 0.889 & 0.013 & 102.3 & 1.1 & & & & & \\
\hline $10118+4222$ & POP116 & 2011.258 & 1 & 0.571 & 0.009 & 72.2 & 1.0 & & & & & \\
\hline $10121+2118$ & A 2146 & 2011.179 & 1 & 0.240 & 0.013 & 176.8 & 2.7 & & & Hei2001 & 0.06 & -24.2 \\
\hline $10121+2118$ & A 2146 & 2012.200 & 1 & 0.213 & 0.013 & $181.7^{*}$ & 1.7 & & & Hei2001 & 0.03 & -23.9 \\
\hline $10125+1552$ & HDS1470 & 2012.197 & 1 & 0.340 & 0.007 & $60.5^{*}$ & 1.3 & & & & & \\
\hline $10125+1552$ & HDS1470 & 2011.135 & 1 & 0.339 & 0.010 & 60.1 & 2.8 & & & & & \\
\hline $10125+1942$ & COU288 & 2011.176 & 1 & 0.615 & 0.057 & 3.3 & 0.7 & & & & & \\
\hline $10126+4901$ & HDS1471 & 2011.258 & 1 & 0.242 & 0.052 & 73.3 & 4.1 & & & & & \\
\hline $10126+4901$ & HDS1471 & 2011.264 & 1 & 0.206 & 0.007 & 65.7 & 3.0 & & & & & \\
\hline $10131+2725$ & STT213 & 2011.176 & 1 & 1.137 & 0.026 & 122.7 & 0.6 & & & Izm2019 & 0.05 & 0.6 \\
\hline $10132+0653$ & HEI757 & 2011.266 & 1 & 1.351 & 0.010 & 44.0 & 0.9 & & & & & \\
\hline $10132+1147$ & HEI355 & 2011.179 & 1 & 0.342 & 0.007 & 24.8 & 0.4 & & & & & \\
\hline $10132+1147$ & HEI355 & 2011.184 & 1 & 0.325 & 0.015 & 25.2 & 1.1 & & & & & \\
\hline $10139+0246$ & HEI758 & 2011.176 & 1 & 0.730 & 0.018 & 48.2 & 0.7 & & & & & \\
\hline $10140+2227$ & COU169 & 2011.179 & 1 & 0.551 & 0.010 & 152.8 & 1.3 & & & & & \\
\hline $10140+2227$ & COU169 & 2012.200 & 1 & 0.524 & 0.007 & $152.4^{*}$ & 0.9 & & & & & \\
\hline $10140+4313$ & HDS1473 & 2012.246 & 1 & 0.224 & 0.014 & 79.5 & 4.6 & & & & & \\
\hline $10148+0509$ & HDS1477 & 2011.184 & 1 & 0.156 & 0.040 & 173.2 & 8.0 & & & & & \\
\hline $10151+6443$ & HDS1478 & 2012.203 & 1 & 0.801 & 0.007 & $64.3^{*}$ & 0.3 & & & & & \\
\hline $10162+4844$ & HU633 & 2011.253 & 1 & 0.190 & 0.040 & 164.7 & 8.0 & & & & & \\
\hline $10162+6406$ & MLR143 & 2012.203 & 1 & 0.700 & 0.007 & $285.5^{*}$ & 0.3 & & & & & \\
\hline $10178+2000$ & A2149 & 2011.179 & 1 & 0.485 & 0.021 & 25.2 & 1.1 & & & & & \\
\hline $10182+0805$ & A 2765 & 2011.231 & 1 & 0.435 & 0.075 & 38.7 & 16.2 & 0.38 & & & & \\
\hline $10182+0805$ & A 2765 & 2011.231 & 1 & 0.427 & 0.010 & 35.2 & 1.2 & & & & & \\
\hline $10183+0841$ & YSC93 & 2012.241 & 1 & 0.268 & 0.007 & $94.4^{*}$ & 0.8 & & & & & \\
\hline $10183+0841$ & YSC93 & 2012.197 & 1 & 0.290 & 0.007 & 93.4 & 1.0 & & & & & \\
\hline $10184+3730$ & HU875 & 2012.252 & 1 & 1.175 & 0.007 & $74.1^{*}$ & 0.3 & & & & & \\
\hline $10184+3730$ & HU875 & 2012.252 & 1 & 1.169 & 0.007 & 73.6 & 0.4 & & & & & \\
\hline $10184+4346$ & POP117 & 2011.258 & 1 & 0.770 & 0.007 & $263.5^{*}$ & 0.3 & & & & & \\
\hline $10187+2155$ & COU170 & 2012.200 & 1 & 0.856 & 0.007 & $111.8^{*}$ & 0.6 & & & & & \\
\hline $10192+1850$ & COU289 & 2012.246 & 1 & - & - & - & - & & NR & & & \\
\hline $10192+1850$ & COU289 & 2012.197 & 1 & 0.205 & 0.007 & 68.1 & 6.4 & & & & & \\
\hline $10192+2034$ & STF1423 & 2011.135 & 1 & 0.717 & 0.016 & 324.0 & 0.7 & & & WSI2004a & 0.06 & 1.2 \\
\hline $10192+2034$ & STF1423 & 2012.200 & 1 & 0.694 & 0.007 & $321.9^{*}$ & 0.3 & & & WSI2004a & 0.04 & 1.6 \\
\hline
\end{tabular}




\begin{tabular}{|c|c|c|c|c|c|c|c|c|c|c|c|c|}
\hline WDS & Name & Epoch & Bin. & $\begin{array}{c}\rho \\
(")\end{array}$ & $\begin{array}{l}\sigma_{\rho} \\
(")\end{array}$ & $\begin{array}{c}\theta \\
\left({ }^{\circ}\right)\end{array}$ & $\begin{array}{l}\sigma_{\theta} \\
\left(^{\circ}\right)\end{array}$ & $\Delta m$ & Notes & Orbit & $\begin{array}{r}\Delta \rho(\mathrm{O}-\mathrm{C}) \\
(")\end{array}$ & $\begin{array}{r}\Delta \theta(\mathrm{O}-\mathrm{C}) \\
\left({ }^{\circ}\right)\end{array}$ \\
\hline $10210+1809$ & COU50 & 2011.179 & 1 & 0.151 & 0.040 & 178.1 & 8.0 & & & & & \\
\hline $10210+1809$ & COU50 & 2011.179 & 1 & - & - & - & - & & $\mathrm{NR}$ & & & \\
\hline $10213+5023$ & HDS1489 & 2011.264 & 1 & 0.244 & 0.007 & $36.8^{*}$ & 1.9 & & & & & \\
\hline $10213+5023$ & HDS1489 & 2011.258 & 1 & - & - & - & - & & $\mathrm{NR}$ & & & \\
\hline $10215+4603$ & COU2503 & 2012.252 & 1 & 1.485 & 0.007 & $172.4^{*}$ & $* 0.5$ & & & & & \\
\hline $10221+1226$ & BU1321 & 2011.228 & 1 & 2.024 & 0.013 & 131.2 & 0.5 & & & & & \\
\hline $10225+0408$ & HEI760 & 2011.266 & 1 & 0.832 & 0.007 & $10.9^{*}$ & 0.4 & & & & & \\
\hline $10225+5030$ & HDS1493 & 2011.264 & 1 & 0.213 & 0.018 & 38.1 & 2.5 & & & & & \\
\hline $10227+1521$ & STT216 & 2012.323 & 2 & 2.246 & 0.022 & $233.0^{*}$ & * 0.3 & 3.05 & & Sca2009c & 0.01 & 0.3 \\
\hline $10227+3956$ & HDS1494 & 2011.253 & 1 & - & - & - & - & & NR & & & \\
\hline $10236+2617$ & A1991 & 2011.220 & 1 & 1.401 & 0.018 & 9.2 & 1.1 & 0.35 & & & & \\
\hline $10236+2617$ & A1991 & 2011.220 & 1 & 1.422 & 0.016 & 8.7 & 0.5 & & & & & \\
\hline $10249+0431$ & HDS1497AB & 2011.231 & 1 & 0.501 & 0.018 & 68.2 & 4.2 & 0.99 & & & & \\
\hline $10249+0431$ & HDS1497AB & 2011.231 & 1 & 0.477 & 0.019 & 72.3 & 0.7 & & & & & \\
\hline $10238+4935$ & HDS1495Aa,Ab & 2011.253 & 1 & - & - & - & - & & $\mathrm{NR}$ & & & \\
\hline $10238+4935$ & $\mathrm{HDS} 1495 \mathrm{Aa}, \mathrm{Ab}$ & 2012.252 & 1 & 0.121 & 0.080 & 161.8 & 8.7 & & & & & \\
\hline $10238+4935$ & HDS1495Aa,Ab & 2012.252 & 1 & 0.110 & 0.080 & 182.6 & 7.7 & & & & & \\
\hline $10250+2437$ & STF1429 & 2012.279 & 1 & 0.784 & 0.007 & $157.9^{*}$ & * 0.4 & & & Zul1981 & 0.04 & -0.7 \\
\hline $10259+4628$ & COU2504 & 2011.253 & 1 & 0.230 & 0.007 & 93.7 & 3.5 & & & & & \\
\hline $10262+0356$ & BU1280BC & 2011.176 & 1 & 0.714 & 0.018 & 45.5 & 0.4 & & & & & \\
\hline $10265+4603$ & A1992 & 2011.258 & 1 & - & - & - & - & & NR & & & \\
\hline $10269+1713$ & STT217 & 2012.244 & 2 & 0.733 & 0.015 & $149.5^{*}$ & * 0.3 & & & Sca2015b & 0.01 & 1.3 \\
\hline $10269+1931$ & COU292 & 2012.197 & 1 & 0.176 & 0.040 & 58.8 & 8.0 & & & Doc2013e & -0.01 & -5.0 \\
\hline $10269+1931$ & COU292 & 2012.200 & 1 & 0.198 & 0.040 & 47.0 & 8.0 & & & Doc2013e & 0.02 & -16.8 \\
\hline $10271+3143$ & A2151 & 2011.264 & 1 & 1.187 & 0.007 & 75.8 & 0.3 & & & & & \\
\hline $10275+0334$ & STT218 & 2011.176 & 1 & 0.364 & 0.007 & 164.2 & 2.2 & & & USN2002 & -0.02 & -4.5 \\
\hline $10275+4136$ & COU1900 & 2011.253 & 1 & 2.412 & 0.012 & $305.2^{*}$ & * 0.3 & & & & & \\
\hline $10281+4847$ & KUI50 & 2011.253 & 2 & 4.024 & 0.020 & $22.2^{*}$ & 0.3 & & & Dru2014 & 0.05 & 0.3 \\
\hline $10281+4847$ & KUI50 & 2012.252 & 1 & 4.024 & 0.020 & 21.8 & 0.3 & & & Dru2014 & 0.08 & -0.2 \\
\hline $10285+3158$ & TDS591 & 2011.264 & 1 & - & - & - & - & & $\mathrm{NR}$ & & & \\
\hline $10286+1146$ & HEI356 & 2011.184 & 1 & 1.532 & 0.058 & 27.3 & 0.7 & & & & & \\
\hline $10287+4558$ & A1993 & 2011.264 & 1 & 0.313 & 0.023 & 204.4 & 3.6 & & & $\operatorname{Lin} 2017 b$ & 0.04 & 2.4 \\
\hline $10291+0922$ & HEI496 & 2011.266 & 1 & 1.801 & 0.009 & 163.7 & 0.3 & & & & & \\
\hline $10292+1009$ & STT220 & 2012.200 & 1 & 0.556 & 0.007 & 101.2 & 0.8 & & & USN2002 & 0.11 & -16.2 \\
\hline $10294+1211$ & HDS1507 & 2012.241 & 1 & - & - & - & - & & NR & & & \\
\hline $10301+2048$ & STF1439 & 2012.279 & 1 & 1.347 & 0.007 & $72.7^{*}$ & 0.3 & & & Izm2019 & 0.00 & -0.4 \\
\hline $10301+3502$ & HDS1509 & 2012.200 & 1 & 0.160 & 0.040 & 93.3 & 8.0 & & & & & \\
\hline $10302+4532$ & ES1150 & 2011.264 & 1 & 0.802 & 0.007 & $181.1^{*}$ & $* 0.6$ & & & & & \\
\hline $10312+3707$ & HU880 & 2011.184 & 1 & 0.880 & 0.007 & 143.7 & 0.3 & & & & & \\
\hline $10312+3707$ & HU880 & 2011.184 & 1 & 0.903 & 0.015 & 143.8 & 0.7 & & & & & \\
\hline $10320+0831$ & YSC39 & 2012.241 & 1 & 0.746 & 0.007 & $37.6^{*}$ & 0.4 & & & & & \\
\hline $10320+0831$ & YSC39 & 2012.241 & 1 & 0.813 & 0.009 & $37.7^{*}$ & 0.5 & & & & & \\
\hline $10321+4723$ & KU35 & 2011.258 & 1 & 1.244 & 0.007 & $15.4^{*}$ & 0.8 & & & & & \\
\hline $10323+2427$ & HDS1511 & 2012.246 & 1 & 0.131 & 0.040 & 18.5 & 8.0 & & & & & \\
\hline $10324+4632$ & A1994 & 2011.258 & 1 & 1.527 & 0.014 & $82.8^{*}$ & 0.3 & & & & & \\
\hline $10331+2052$ & COU172 & 2011.266 & 1 & 0.255 & 0.011 & 33.2 & 1.3 & & & & & \\
\hline $10337+2045$ & COU173 & 2012.279 & 1 & 0.842 & 0.007 & $185.0^{*}$ & $* 0.6$ & & & & & \\
\hline $10337+2045$ & COU173 & 2012.279 & 1 & 0.837 & 0.011 & $183.1^{*}$ & $* 0.3$ & & & & & \\
\hline $10341+1222$ & CHR31 & 2011.179 & 1 & 0.118 & 0.040 & 168.0 & 8.0 & & & & & \\
\hline $10343+1802$ & YSC40 & 2012.197 & 1 & - & - & - & - & & $\mathrm{NR}$ & & & \\
\hline $10352+4624$ & HDS1517 & 2011.253 & 1 & - & - & - & - & & NR & & & \\
\hline $10355+4155$ & COU1901 & 2011.258 & 1 & 1.409 & 0.019 & $302.0^{*}$ & * 0.3 & & & & & \\
\hline
\end{tabular}




\begin{tabular}{|c|c|c|c|c|c|c|c|c|c|c|c|c|}
\hline WDS & Name & Epoch & Bin. & $\begin{array}{c}\rho \\
(")\end{array}$ & $\begin{array}{l}\sigma_{\rho} \\
(")\end{array}$ & $\begin{array}{c}\theta \\
\left({ }^{\circ}\right)\end{array}$ & $\begin{array}{l}\sigma_{\theta} \\
\left(^{\circ}\right)\end{array}$ & $\Delta m$ & Notes & Orbit & $\begin{array}{r}\Delta \rho(\mathrm{O}-\mathrm{C}) \\
(")\end{array}$ & $\begin{array}{r}\Delta \theta(\mathrm{O}-\mathrm{C}) \\
\left({ }^{\circ}\right)\end{array}$ \\
\hline $10355+4357$ & COU2091 & 2012.246 & 1 & 0.765 & 0.007 & $144.2^{*}$ & 0.5 & & & & & \\
\hline $10358+0233$ & A2571BC & 2011.176 & 1 & 0.329 & 0.014 & 173.9 & 4.6 & & & & & \\
\hline $10358+4937$ & COU2623 & 2011.258 & 1 & 1.808 & 0.036 & 301.2 & 0.5 & & & & & \\
\hline $10358+4937$ & COU2623 & 2011.258 & 1 & 1.794 & 0.023 & $299.2^{*}$ & 0.7 & & & & & \\
\hline $10371+3759$ & COU1571 & 2011.184 & 1 & 1.441 & 0.032 & 114.6 & 0.7 & & & & & \\
\hline $10382+2636$ & STF1454 & 2011.266 & 1 & 1.367 & 0.007 & $354.0^{*}$ & 0.3 & & & Izm2019 & 0.05 & -0.8 \\
\hline $10382+2636$ & STF1454 & 2012.246 & 1 & 1.342 & 0.007 & $354.7^{*}$ & 0.3 & & & Izm2019 & 0.06 & -0.7 \\
\hline $10382+4558$ & COU2092 & 2011.253 & 1 & 0.576 & 0.007 & $281.2^{*}$ & 0.3 & & & & & \\
\hline $10385+3114$ & TDS7389 & 2011.264 & 1 & 0.615 & 0.017 & $317.3^{*}$ & 0.5 & & & & & \\
\hline $10392+4529$ & COU2094 & 2011.253 & 1 & 0.598 & 0.007 & $214.5^{*}$ & 0.3 & & & & & \\
\hline $10397+0851$ & STT224 & 2011.135 & 1 & 0.508 & 0.021 & 141.0 & 1.7 & & & Hrt2010a & -0.00 & 0.9 \\
\hline $10401+1914$ & PER2AB & 2011.220 & 1 & 0.453 & 0.013 & 39.6 & 2.1 & & & & & \\
\hline $10401+1914$ & PER2AB & 2011.220 & 1 & 0.438 & 0.012 & 41.9 & 3.2 & & & & & \\
\hline $10406+4700$ & HDS1525 & 2011.253 & 1 & 0.216 & 0.033 & $225.6^{*}$ & 1.8 & & & & & \\
\hline $10408+2657$ & COU779 & 2012.197 & 1 & 0.393 & 0.007 & $83.5^{*}$ & 1.8 & & & & & \\
\hline $10417+1044$ & STT227 & 2011.272 & 1 & 0.908 & 0.007 & $3.9^{*}$ & 0.3 & & & & & \\
\hline $10426+0335$ & A2768 & 2011.135 & 1 & 0.606 & 0.025 & 251.2 & 2.5 & & & Tok2015c & 0.00 & 2.2 \\
\hline $10428+4711$ & COU2096 & 2011.253 & 1 & 0.328 & 0.022 & $273.8^{*}$ & 1.2 & & & & & \\
\hline $10432+0440$ & A2769 & 2011.231 & 1 & 0.487 & 0.034 & 25.3 & 4.7 & 1.46 & & & & \\
\hline $10432+0440$ & A2769 & 2011.231 & 1 & 0.483 & 0.007 & 26.5 & 0.6 & & & & & \\
\hline $10442+3955$ & YR26 & 2011.220 & 1 & 0.531 & 0.027 & 196.1 & 3.8 & & & & & \\
\hline $10442+3955$ & YR26 & 2012.200 & 1 & 0.528 & 0.014 & $198.4^{*}$ & 1.6 & & & & & \\
\hline $10442+4441$ & HDS1533 & 2011.264 & 1 & 0.430 & 0.013 & $231.4^{*}$ & 2.3 & & & & & \\
\hline $10446+0402$ & A 2770 & 2011.231 & 1 & 0.879 & 0.028 & 19.2 & 3.1 & & & & & \\
\hline $10446+0402$ & A 2770 & 2011.231 & 1 & 0.844 & 0.021 & 15.9 & 2.8 & & & & & \\
\hline $10446+0402$ & A 2770 & 2011.231 & 1 & 0.854 & 0.017 & 17.6 & 0.3 & & & & & \\
\hline $10446+0530$ & A 2771 & 2011.135 & 1 & 0.410 & 0.049 & 118.7 & 3.2 & & & Tok2014a & -0.00 & -2.0 \\
\hline $10454+0759$ & HDS1537 & 2011.184 & 1 & 1.085 & 0.012 & 97.3 & 0.4 & & & & & \\
\hline $10454+3831$ & $\mathrm{HO} 532 \mathrm{AC}$ & 2011.176 & 1 & 0.690 & 0.010 & 235.9 & 1.6 & & & Tok2019e & 0.02 & -1.7 \\
\hline $10454+3831$ & $\mathrm{HO} 532 \mathrm{AC}$ & 2011.179 & 1 & 0.690 & 0.015 & 238.7 & 2.7 & & & Tok2019e & 0.02 & 1.1 \\
\hline $10454+3831$ & $\mathrm{HO} 532 \mathrm{AC}$ & 2012.200 & 1 & 0.682 & 0.007 & 235.8 & 2.3 & & & Tok2019e & 0.01 & -1.5 \\
\hline $10454+3831$ & $\mathrm{HO} 532 \mathrm{AC}$ & 2012.323 & 1 & 0.699 & 0.007 & 236.6 & 2.3 & 2.67 & & Tok2019e & 0.02 & -0.5 \\
\hline $10455+4541$ & COU2097 & 2011.253 & 1 & 1.135 & 0.010 & $338.6^{*}$ & 0.5 & & & & & \\
\hline $10463+2537$ & HDS1539 & 2012.200 & 1 & 0.414 & 0.009 & $58.1^{*}$ & 1.0 & & & & & \\
\hline $10463+2537$ & HDS1539 & 2011.272 & 1 & 0.421 & 0.009 & $59.9^{*}$ & 0.7 & & & & & \\
\hline $10472+2605$ & COU591 & 2011.135 & 1 & 0.452 & 0.013 & 4.7 & 1.3 & & & & & \\
\hline $10477+2733$ & COU592 & 2012.197 & 1 & 0.424 & 0.007 & $211.6^{*}$ & 0.3 & & & & & \\
\hline $10480+4107$ & STT229 & 2011.258 & 1 & 0.676 & 0.007 & $260.9^{*}$ & 0.3 & & & Alz2020c & 0.00 & -0.5 \\
\hline $10480+4107$ & STT229 & 2012.323 & 2 & 0.684 & 0.007 & 261.5 & 0.3 & 0.64 & & $\mathrm{Alz2020 \textrm {c }}$ & 0.01 & 0.9 \\
\hline $10496+3948$ & A 2155 & 2011.184 & 1 & 0.915 & 0.034 & 16.9 & 2.1 & & & & & \\
\hline $10497+2417$ & BU915 & 2011.266 & 1 & 1.489 & 0.007 & 53.3 & 0.3 & & & & & \\
\hline $10510+0502$ & HDS1549 & 2011.184 & 1 & 0.454 & 0.015 & 171.4 & 1.4 & & & & & \\
\hline $10510+1603$ & A 2372 & 2012.200 & 1 & 0.358 & 0.007 & $97.8^{*}$ & 1.3 & & & & & \\
\hline $10511+1135$ & CHR32 & 2011.228 & 1 & 0.778 & 0.012 & 149.1 & 1.8 & & & & & \\
\hline $10515+0756$ & HDS1550 & 2011.231 & 1 & 0.694 & 0.013 & 5.0 & 1.0 & & & & & \\
\hline $10520+1606$ & A 2373 & 2012.200 & 1 & 0.208 & 0.010 & $35.7^{*}$ & 0.7 & & & USN2007a & 0.01 & $-6.0^{Q}$ \\
\hline $10539+1706$ & HDS1559 & 2011.179 & 1 & 0.487 & 0.007 & 154.0 & 2.1 & & & & & \\
\hline $10543+0737$ & HDS1560 & 2011.184 & 1 & 0.549 & 0.010 & 57.9 & 1.0 & & & & & \\
\hline $10543+2607$ & A1769 & 2011.220 & 1 & 0.706 & 0.007 & 83.9 & 2.7 & 0.23 & & & & \\
\hline $10543+2607$ & A1769 & 2011.220 & 1 & 0.677 & 0.035 & 85.8 & 1.4 & & & & & \\
\hline
\end{tabular}




\begin{tabular}{|c|c|c|c|c|c|c|c|c|c|c|c|c|}
\hline WDS & Name & Epoch & Bin. & $\begin{array}{c}\rho \\
(")\end{array}$ & $\begin{array}{l}\sigma_{\rho} \\
(")\end{array}$ & $\begin{array}{c}\theta \\
\left(^{\circ}\right)\end{array}$ & $\begin{array}{l}\sigma_{\theta} \\
\left({ }^{\circ}\right)\end{array}$ & $\Delta m$ & Notes & Orbit & $\begin{array}{r}\Delta \rho(\mathrm{O}-\mathrm{C}) \\
(")\end{array}$ & $\begin{array}{r}\Delta \theta(\mathrm{O}-\mathrm{C}) \\
\left({ }^{\circ}\right)\end{array}$ \\
\hline $10544+3840$ & COU1746 & 2011.176 & 1 & 0.367 & 0.018 & 143.4 & 1.5 & & & $\operatorname{Lin} 2018 a$ & -0.01 & 0.1 \\
\hline $10544+3840$ & COU1746 & 2012.200 & 1 & 0.376 & 0.017 & $145.3^{*}$ & ${ }^{*} 0.6$ & & & $\operatorname{Lin} 2018 \mathrm{a}$ & 0.00 & 0.9 \\
\hline $10548+2345$ & BU597 & 2012.323 & 1 & 1.028 & 0.040 & $45.4^{*}$ & 0.3 & 1.41 & & & & \\
\hline $10564+3338$ & COU1421 & 2012.279 & 1 & 1.962 & 0.334 & $96.1^{*}$ & 2.0 & & & & & \\
\hline $10564+3652$ & COU1420 & 2012.200 & 1 & 0.150 & 0.040 & 24.8 & 8.0 & & & & & \\
\hline $10567+3320$ & POP73 & 2011.220 & 1 & 0.769 & 0.037 & 23.4 & 0.9 & 0.43 & & & & \\
\hline $10567+3444$ & HU726 & 2011.228 & 1 & 1.150 & 0.007 & 103.2 & 0.3 & & & & & \\
\hline $10567+3456$ & POP112 & 2011.258 & 2 & 1.405 & 0.015 & $243.6^{*}$ & ${ }^{*} 0.9$ & & & & & \\
\hline $10585+1711$ & A 2375 & 2011.135 & 1 & 0.524 & 0.018 & 172.7 & 3.3 & & & Doc2009g & 0.05 & -1.3 \\
\hline $10585+1711$ & A 2375 & 2012.200 & 1 & 0.491 & 0.016 & 173.9 & 0.9 & & & Doc $2009 \mathrm{~g}$ & 0.01 & 1.0 \\
\hline $10588+0759$ & TDS7584 & 2011.266 & 1 & - & - & - & - & & NR & & & \\
\hline $10596+0956$ & A2774 & 2011.184 & 1 & 1.811 & 0.024 & 108.5 & 0.6 & & & & & \\
\hline $11003+1926$ & A2376 & 2011.135 & 1 & 0.128 & 0.040 & 183.1 & 8.0 & & & & & \\
\hline $11008+2913$ & COU960 & 2011.266 & 1 & 0.220 & 0.009 & 295.6 & 2.1 & & & & & \\
\hline $11008+2913$ & COU960 & 2011.266 & 1 & 0.212 & 0.009 & 294.1 & 2.6 & & & & & \\
\hline $11008+2913$ & COU960 & 2012.200 & 1 & 0.199 & 0.040 & $300.0^{*}$ & ${ }^{*} 6.0$ & & & & & \\
\hline $11009+4036$ & COU1747 & 2011.253 & 1 & 0.828 & 0.013 & $315.1^{*}$ & ${ }^{*} 0.3$ & & & & & \\
\hline $11014+1235$ & HEI155 & 2011.179 & 1 & 0.746 & 0.016 & 67.5 & 0.8 & & & & & \\
\hline $11017+3641$ & COU1422Ba,Bb & 2012.200 & 1 & 0.630 & 0.013 & $41.8^{*}$ & 0.8 & & & Tok $2019 \mathrm{e}$ & 0.00 & -2.0 \\
\hline $11017+3641$ & HDS1574Aa,Ab & 2012.200 & 1 & - & - & - & - & & NR & & & \\
\hline $11018+1903$ & HDS1575 & 2011.179 & 1 & 0.264 & 0.017 & 109.2 & 1.9 & & & & & \\
\hline $11018+2952$ & COU961 & 2012.323 & 1 & 2.349 & 0.012 & $327.8^{*}$ & ${ }^{*} 0.3$ & 1.40 & & & & \\
\hline $11027+4446$ & COU2098 & 2011.253 & 1 & 1.710 & 0.009 & $184.6^{*}$ & ${ }^{*} 0.3$ & & & & & \\
\hline $11029+3541$ & $\mathrm{HO} 47 \mathrm{BC}$ & 2011.220 & 1 & 0.877 & 0.023 & 158.6 & 2.6 & 0.28 & & & & \\
\hline $11029+3541$ & $\mathrm{HO} 47 \mathrm{BC}$ & 2011.220 & 1 & 0.912 & 0.027 & 156.5 & 2.3 & & & & & \\
\hline $11032+2142$ & COU175 & 2012.323 & 1 & 1.156 & 0.042 & $294.8^{*}$ & ${ }^{*} 0.3$ & 2.19 & & & & \\
\hline $11053+1635$ & A 2378 & 2012.246 & 1 & 0.384 & 0.007 & $324.1^{*}$ & ${ }^{*} 1.1$ & & & & & \\
\hline $11055+3927$ & COU1748 & 2011.228 & 1 & 0.171 & 0.040 & 73.2 & 8.0 & & & & & \\
\hline $11055+3927$ & COU1748 & 2012.279 & 1 & 0.176 & 0.040 & 90.4 & 8.0 & & & & & \\
\hline $11065+2653$ & A1775 & 2012.246 & 1 & 0.223 & 0.007 & 87.9 & 0.4 & & & & & \\
\hline $11069+3746$ & COU1572 & 2012.200 & 1 & 1.884 & 0.009 & $183.8^{*}$ & ${ }^{*} 0.3$ & & & & & \\
\hline $11075+3857$ & HDS1585 & 2011.179 & 1 & 0.366 & 0.008 & 7.6 & 0.8 & & & & & \\
\hline $11090+4325$ & COU2099 & 2011.253 & 1 & 0.275 & 0.021 & 102.8 & 2.2 & & & & & \\
\hline $11100+2138$ & COU176 & 2011.135 & 1 & 0.812 & 0.017 & 75.8 & 1.4 & & & & & \\
\hline $11104+1110$ & HDS1591 & 2011.184 & 1 & 0.624 & 0.007 & 42.5 & 0.5 & & & & & \\
\hline $11104+1502$ & HEI61 & 2011.231 & 1 & 0.266 & 0.015 & 86.6 & 1.1 & & & & & \\
\hline $11104+1502$ & HEI61 & 2011.231 & 1 & - & - & - & - & & NR & & & \\
\hline $11107+3110$ & HJ2562 & 2011.179 & 1 & 1.015 & 0.016 & 228.6 & 0.6 & & & Izm2019 & -0.06 & -4.1 \\
\hline $11107+3110$ & HJ2562 & 2012.200 & 1 & 1.004 & 0.007 & $228.3^{*}$ & ${ }^{*} 0.3$ & & & Izm2019 & -0.07 & -3.4 \\
\hline $11114+4150$ & HDS1593 & 2011.253 & 1 & 0.337 & 0.033 & $157.3^{*}$ & ${ }^{*} 0.9$ & & & & & \\
\hline $11120+3500$ & A2156 & 2012.279 & 1 & 0.207 & 0.007 & $168.4^{*}$ & ${ }^{*} 2.1$ & & & & & \\
\hline $11131+1646$ & COU295 & 2011.264 & 1 & 0.238 & 0.013 & $168.5^{*}$ & ${ }^{*} 5.0$ & & & & & \\
\hline $11137+2008$ & STF1517 & 2011.220 & 1 & 0.637 & 0.008 & 317.5 & 1.1 & & & FMR2015b & -0.03 & 0.4 \\
\hline $11143+3553$ & ES2165 & 2011.264 & 1 & 1.976 & 0.010 & $92.7^{*}$ & 0.3 & & & & & \\
\hline $11144+1531$ & BU1283 & 2011.231 & 1 & 0.316 & 0.013 & 175.1 & 0.9 & & & & & \\
\hline $11144+1531$ & BU1283 & 2012.279 & 1 & 0.275 & 0.080 & $182.0^{*}$ & ${ }^{*} 5.7$ & & & & & \\
\hline $11151+3735$ & STT232 & 2012.350 & 1 & 0.618 & 0.007 & 64.2 & 0.4 & & & & & \\
\hline $11155+4729$ & HU639 & 2011.253 & 1 & 0.254 & 0.007 & $89.8^{*}$ & 0.7 & & & & & \\
\hline $11162+3136$ & A2157 & 2012.247 & 1 & 1.317 & 0.010 & 182.3 & 0.3 & 2.49 & & Pop1996b & 0.24 & -55.6 \\
\hline $11162+3136$ & A 2157 & 2012.247 & 1 & 1.325 & 0.008 & 182.2 & 0.3 & & & Pop1996b & 0.25 & -55.5 \\
\hline $11162+3136$ & A 2157 & 2012.323 & 2 & 1.335 & 0.007 & 182.8 & 0.3 & 3.76 & & Pop1996b & 0.26 & -54.8 \\
\hline $11163+0915$ & HDS1606 & 2011.231 & 1 & 0.618 & 0.042 & 87.0 & 1.7 & & & & & \\
\hline $11172+0745$ & HDS1609 & 2011.184 & 1 & 0.951 & 0.012 & 16.0 & 0.6 & & & & & \\
\hline
\end{tabular}




\begin{tabular}{|c|c|c|c|c|c|c|c|c|c|c|c|c|}
\hline WDS & Name & Epoch & Bin. & $\begin{array}{c}\rho \\
(")\end{array}$ & $\begin{array}{l}\sigma_{\rho} \\
(")\end{array}$ & $\begin{array}{c}\theta \\
\left({ }^{\circ}\right)\end{array}$ & $\begin{array}{l}\sigma_{\theta} \\
\left({ }^{\circ}\right)\end{array}$ & $\Delta m$ & Notes & Orbit & $\begin{array}{r}\Delta \rho(\mathrm{O}-\mathrm{C}) \\
(")\end{array}$ & $\begin{array}{r}\Delta \theta(\mathrm{O}-\mathrm{C}) \\
\left({ }^{\circ}\right)\end{array}$ \\
\hline $11174+4146$ & A 2158 & 2011.253 & 1 & 0.428 & 0.007 & $182.9^{*}$ & 0.8 & & & & & \\
\hline $11182+1638$ & A2379BC & 2011.264 & 1 & 0.416 & 0.007 & 103.6 & 0.4 & & & & & \\
\hline $11190+1416$ & STF1527 & 2012.279 & 1 & 0.323 & 0.016 & $202.9^{*}$ & 3.5 & & & Tok2012b & 0.01 & -6.7 \\
\hline $11196+4930$ & HDS1615 & 2011.253 & 1 & 0.145 & 0.040 & 25.7 & 8.0 & & & & & \\
\hline $11208+2402$ & TDS7805 & 2011.272 & 1 & 0.847 & 0.007 & $319.8^{*}$ & 0.7 & & & & & \\
\hline $11218+4520$ & A1847 & 2011.253 & 1 & 1.387 & 0.007 & $329.4^{*}$ & 0.3 & & & & & \\
\hline $11218+4520$ & A1847 & 2011.253 & 1 & 1.389 & 0.007 & $329.5^{*}$ & 0.3 & & & & & \\
\hline $11221+3705$ & COU1260 & 2012.279 & 1 & 0.398 & 0.007 & $12.5^{*}$ & 0.7 & & & & & \\
\hline $11225+2250$ & TDS7831 & 2011.272 & 1 & - & - & - & - & & NR & & & \\
\hline $11230+0408$ & $\mathrm{~A} 2776 \mathrm{AB}$ & 2011.231 & 1 & 0.161 & 0.040 & 40.5 & 6.7 & & & Hrt2010a & -0.00 & -16.2 \\
\hline $11235+4828$ & COU2100 & 2011.253 & 1 & 1.590 & 0.008 & $23.5^{*}$ & 0.3 & & & & & \\
\hline $11241+2929$ & TDS7840 & 2011.264 & 1 & 1.379 & 0.012 & $202.4^{*}$ & 0.5 & & & & & \\
\hline $11247+3758$ & COU1261 & 2012.200 & 1 & 0.669 & 0.007 & $219.1^{*}$ & 0.3 & & & & & \\
\hline $11254+0607$ & HDS1624 & 2011.184 & 1 & 0.520 & 0.024 & 11.5 & 1.8 & & & & & \\
\hline $11260+1213$ & YSC41 & 2012.279 & 1 & 1.172 & 0.007 & $276.1^{*}$ & 0.3 & & & & & \\
\hline $11263+1610$ & HEI157 & 2012.323 & 1 & 1.605 & 0.046 & 156.7 & 0.3 & 1.75 & & & & \\
\hline $11265+0806$ & A 2575 & 2011.184 & 1 & 0.444 & 0.007 & 46.5 & 2.1 & & & USN2002 & 0.19 & -16.4 \\
\hline $11271+3546$ & HDS1626 & 2011.179 & 1 & 0.272 & 0.046 & 97.5 & 1.0 & & & & & \\
\hline $11271+3546$ & HDS1626 & 2012.350 & 1 & 0.261 & 0.020 & 90.4 & 3.5 & & & & & \\
\hline $11281+4055$ & TDS7870 & 2012.323 & 1 & - & - & - & - & & NR & & & \\
\hline $11287+3224$ & COU781 & 2011.253 & 1 & 0.604 & 0.007 & $289.0^{*}$ & 0.3 & & & & & \\
\hline $11290+1555$ & HDS1629 & 2011.179 & 1 & 0.459 & 0.011 & 142.1 & 1.8 & & & & & \\
\hline $11290+1555$ & HDS1629 & 2011.179 & 1 & 0.475 & 0.008 & 141.1 & 1.2 & & & & & \\
\hline $11290+1555$ & HDS1629 & 2011.184 & 1 & 0.462 & 0.007 & 140.8 & 0.7 & & & & & \\
\hline $11292+0606$ & HEI851 & 2011.231 & 1 & 0.953 & 0.013 & 144.3 & 0.4 & & & & & \\
\hline $11292+0606$ & HEI851 & 2011.231 & 1 & 0.940 & 0.025 & 142.7 & 4.1 & 3.48 & & & & \\
\hline $11293+3025$ & L11 & 2011.264 & 1 & 1.013 & 0.007 & $281.7^{*}$ & 0.3 & & & RAO2015 & -0.01 & -1.6 \\
\hline $11294+2633$ & TDS7887 & 2011.272 & 1 & - & - & - & - & & NR & & & \\
\hline $11301+2237$ & A2484 & 2012.350 & 1 & 0.461 & 0.015 & 161.6 & 0.6 & & & & & \\
\hline $11308+4117$ & STT234 & 2011.253 & 1 & 0.470 & 0.007 & $172.7^{*}$ & 0.3 & & & Doc $2009 \mathrm{~g}$ & -0.00 & -1.1 \\
\hline $11308+4117$ & STT234 & 2012.323 & 1 & 0.476 & 0.007 & 173.3 & 0.3 & 0.41 & & Doc $2009 \mathrm{~g}$ & 0.01 & -2.0 \\
\hline $11319+4555$ & COU1750 & 2011.258 & 2 & 2.012 & 0.027 & $233.1^{*}$ & 0.4 & & & & & \\
\hline $11322+3615$ & HU1134 & 2011.220 & 1 & 0.200 & 0.008 & 111.8 & 10.1 & & & Hrt2000b & 0.17 & $-13.0^{Q}$ \\
\hline $11322+3615$ & HU1134 & 2012.337 & 1 & 0.152 & 0.040 & $119.6^{*}$ & 8.0 & & & Hrt2000b & 0.09 & $-3.0^{Q}$ \\
\hline $11323+2527$ & TDS7912 & 2011.264 & 1 & 0.516 & 0.049 & 174.9 & 0.5 & & & & & \\
\hline $11332+4927$ & HU727 & 2011.258 & 2 & 1.224 & 0.015 & $205.4^{*}$ & 0.4 & & & & & \\
\hline $11334+4637$ & COU1751 & 2011.253 & 1 & 0.166 & 0.040 & 140.5 & 8.0 & & & & & \\
\hline $11335+0759$ & HDS1639 & 2011.184 & 1 & 0.221 & 0.011 & 170.0 & 2.4 & & & & & \\
\hline $11336+3702$ & HJ502 & 2012.337 & 1 & 1.349 & 0.007 & 285.0 & 0.3 & 2.03 & & & & \\
\hline $11336+4729$ & COU1573 & 2011.258 & 1 & 0.300 & 0.019 & $91.7^{*}$ & 1.1 & & & & & \\
\hline $11352+3524$ & HU887 & 2012.337 & 1 & 1.283 & 0.007 & 304.4 & 0.3 & 1.90 & & & & \\
\hline $11355+3452$ & HDS1644 & 2012.279 & 1 & - & - & - & - & & NR & & & \\
\hline $11361+1251$ & STF1554 & 2012.279 & 1 & 0.283 & 0.007 & $205.8^{*}$ & 1.9 & & & Zir2011b & -0.01 & -9.4 \\
\hline $11363+2747$ & STF1555 & 2012.279 & 1 & 0.723 & 0.007 & $149.7^{*}$ & 0.5 & & & Doc2017a & 0.01 & 0.3 \\
\hline $11367+2128$ & STF1558 & 2011.220 & 2 & 0.681 & 0.037 & 165.4 & 1.0 & & & & & \\
\hline $11367+2128$ & STF1558 & 2011.220 & 1 & 0.637 & 0.016 & 165.8 & 1.3 & 0.74 & & & & \\
\hline $11374+4728$ & KU39 & 2011.253 & 1 & 1.212 & 0.007 & $132.6^{*}$ & 0.3 & & & Izm2019 & 0.04 & 1.4 \\
\hline $11389+4733$ & COU1574 & 2011.258 & 2 & 1.724 & 0.031 & $184.3^{*}$ & 0.3 & & & & & \\
\hline $11390+4109$ & STT237 & 2012.323 & 1 & 2.044 & 0.010 & $244.0^{*}$ & 0.3 & 1.19 & & USN2002 & 0.02 & -0.7 \\
\hline $11392+2640$ & HDS1651 & 2011.184 & 1 & 0.879 & 0.018 & 38.4 & 1.5 & & & & & \\
\hline $11401+2906$ & TDS7979 & 2011.264 & 1 & - & - & - & - & & NR & & & \\
\hline $11425+2355$ & COU390 & 2011.179 & 1 & 0.287 & 0.049 & 68.3 & 5.1 & & & Tok2016e & 0.05 & 1.9 \\
\hline $11425+2355$ & COU390 & 2011.179 & 1 & 0.244 & 0.051 & 69.6 & 1.7 & & & Tok2016e & 0.01 & 3.2 \\
\hline $11428+2105$ & HU888 & 2012.323 & 1 & 0.554 & 0.047 & $177.9^{*}$ & 0.3 & 0.95 & & & & \\
\hline
\end{tabular}




\begin{tabular}{|c|c|c|c|c|c|c|c|c|c|c|c|c|}
\hline WDS & Name & Epoch & Bin. & $\begin{array}{c}\rho \\
(")\end{array}$ & $\begin{array}{l}\sigma_{\rho} \\
(")\end{array}$ & $\begin{array}{c}\theta \\
\left({ }^{\circ}\right)\end{array}$ & $\begin{array}{l}\sigma_{\theta} \\
\left({ }^{\circ}\right)\end{array}$ & $\Delta m$ & Notes & Orbit & $\begin{array}{r}\Delta \rho(\mathrm{O}-\mathrm{C}) \\
(")\end{array}$ & $\begin{array}{r}\Delta \theta(\mathrm{O}-\mathrm{C}) \\
\left({ }^{\circ}\right)\end{array}$ \\
\hline $11431+3715$ & HU1135 & 2012.279 & 1 & 0.689 & 0.007 & $335.2^{*}$ & 0.3 & & & & & \\
\hline $11434+2245$ & HJ2581 & 2012.337 & 1 & - & - & - & - & & NR & & & \\
\hline $11461+3727$ & ES1738 & 2012.337 & 1 & 1.879 & 0.009 & 164.5 & 0.3 & 1.27 & & & & \\
\hline $11463+3303$ & A 2160 & 2012.350 & 1 & 0.134 & 0.063 & 173.2 & 7.4 & & & & & \\
\hline $11475+2002$ & HDS1665 & 2012.323 & 1 & 2.033 & 0.010 & $133.0^{*}$ & 0.3 & 2.96 & & & & \\
\hline $11476+1514$ & TDS646 & 2011.264 & 1 & 0.719 & 0.016 & 172.6 & 0.3 & & & & & \\
\hline $11478+4949$ & HU729 & 2012.362 & 1 & 1.506 & 0.008 & 347.6 & 0.3 & 3.56 & & & & \\
\hline $11478+4949$ & HU729 & 2012.361 & 1 & 1.506 & 0.008 & $347.4^{*}$ & 0.3 & & & & & \\
\hline $11485+1633$ & HEI158 & 2011.220 & 2 & 1.451 & 0.053 & 100.9 & 1.3 & & & & & \\
\hline $11485+3507$ & TDS8038 & 2011.258 & 2 & 1.925 & 0.015 & 6.4 & 0.6 & & & & & \\
\hline $11487+3937$ & COU1263 & 2012.350 & 1 & 0.376 & 0.013 & 57.2 & 0.3 & & & & & \\
\hline $11487+4030$ & COU1262 & 2011.253 & 1 & 0.684 & 0.007 & $247.8^{*}$ & 0.4 & & & & & \\
\hline $11499+3645$ & HDS1669 & 2011.179 & 1 & 0.110 & 0.048 & 100.1 & 6.9 & & & & & \\
\hline $11499+3754$ & COU1129 & 2012.323 & 1 & 0.544 & 0.047 & $124.1^{*}$ & 0.3 & 1.92 & & & & \\
\hline $11503+3741$ & HDS1670 & 2012.337 & 1 & 1.258 & 0.007 & 21.1 & 0.3 & 2.29 & & & & \\
\hline $11504+3544$ & COU964 & 2011.179 & 1 & 1.418 & 0.007 & 60.0 & 0.3 & & & & & \\
\hline $11509+2557$ & TDS8067 & 2011.264 & 1 & 1.851 & 0.019 & $56.4^{*}$ & 0.3 & & & & & \\
\hline $11510+3653$ & HU889 & 2012.323 & 1 & 0.977 & 0.050 & $294.8^{*}$ & 0.3 & 1.45 & & & & \\
\hline $11511+2245$ & HDS1671 & 2012.361 & 1 & 0.652 & 0.007 & $68.2^{*}$ & 0.4 & & & & & \\
\hline $11512+3629$ & TDS8069 & 2011.264 & 1 & - & - & - & - & & NR & & & \\
\hline $11514+1148$ & HDS1672 & 2011.272 & 1 & - & - & - & - & & NR & & & \\
\hline $11517+2344$ & COU391 & 2012.337 & 1 & 0.323 & 0.007 & 300.8 & 0.3 & 0.88 & & & & \\
\hline $11524+1428$ & HDS1675 & 2011.184 & 1 & 0.103 & 0.040 & 46.4 & 12.1 & & & & & \\
\hline $11537+2626$ & HDS1677 & 2011.228 & 1 & 0.358 & 0.009 & 60.1 & 1.7 & & & & & \\
\hline $11544+1515$ & WOR20 & 2011.264 & 1 & 1.212 & 0.007 & $290.5^{*}$ & 0.3 & & & & & \\
\hline $11544+1515$ & WOR20 & 2012.323 & 0 & 1.205 & 0.006 & 290.4 & 0.3 & & & & & \\
\hline $11544+1515$ & WOR20 & 2012.323 & 1 & 1.190 & 0.007 & 291.2 & 0.7 & & & & & \\
\hline $11551+4629$ & $\mathrm{~A} 1777 \mathrm{AB}$ & 2011.253 & 1 & 0.272 & 0.014 & 339.4 & 1.2 & & & Zas2012b & 0.06 & 3.1 \\
\hline $11551+4629$ & $\mathrm{~A} 1777 \mathrm{AC}$ & 2011.253 & 1 & 3.905 & 0.020 & $41.8^{*}$ & 0.3 & 2.46 & & & & ND \\
\hline $11551+4629$ & $\mathrm{~A} 1777 \mathrm{AC}$ & 2011.253 & 1 & 3.900 & 0.020 & $41.7^{*}$ & 0.3 & & & & & $\mathrm{ND}$ \\
\hline $11552+1643$ & TDS8112 & 2012.337 & 1 & 1.241 & 0.007 & 162.8 & 0.3 & 0.24 & & & & \\
\hline $11552+1643$ & TDS8112 & 2011.264 & 1 & 1.232 & 0.008 & $163.5^{*}$ & 0.3 & & & & & \\
\hline $11572+3312$ & COU939 & 2011.253 & 1 & 0.232 & 0.011 & $33.3^{*}$ & 2.0 & & & & & \\
\hline $11572+3312$ & COU939 & 2012.350 & 1 & 0.269 & 0.015 & 27.4 & 1.9 & & & & & \\
\hline $11574+1823$ & A2486 & 2012.279 & 1 & 1.128 & 0.007 & $239.8^{*}$ & 0.8 & & & & & \\
\hline $11575+2818$ & TDS8142 & 2011.264 & 1 & - & - & - & - & & NR & & & \\
\hline $11576+1742$ & COU296 & 2012.279 & 1 & - & - & - & - & & NR & & & \\
\hline $11591+0601$ & HDS1687 & 2011.231 & 1 & 0.426 & 0.027 & 121.5 & 0.3 & & & & & \\
\hline $11591+0601$ & HDS1687 & 2011.231 & 1 & 0.497 & 0.048 & 123.4 & 3.2 & 0.45 & & & & \\
\hline $12003+2714$ & TDS8158 & 2011.272 & 2 & - & - & - & - & & NR & & & \\
\hline $12006+1820$ & HDS1688 & 2012.337 & 1 & 0.426 & 0.007 & 9.6 & 0.3 & 2.11 & & & & \\
\hline $12007+2905$ & TDS8162 & 2011.272 & 2 & 0.497 & 0.034 & 12.2 & 6.3 & & & & & \\
\hline $12010+1719$ & TDS8164 & 2011.272 & 2 & - & - & - & - & & NR & & & \\
\hline $12015+3444$ & HDS1689 & 2012.361 & 1 & 0.136 & 0.040 & 36.9 & 8.0 & & & & & \\
\hline $12016+0617$ & HEI507 & 2011.231 & 1 & 1.447 & 0.032 & 40.2 & 1.1 & & & & & \\
\hline $12016+0617$ & HEI507 & 2011.231 & 1 & 1.529 & 0.035 & 39.3 & 1.0 & 2.42 & & & & \\
\hline $12017+4728$ & COU1752 & 2012.361 & 1 & - & - & - & - & & NR & & & \\
\hline $12025+2128$ & TDS8185 & 2011.264 & 1 & - & - & - & - & & NR & & & \\
\hline $12032+1709$ & COU177 & 2012.337 & 1 & 1.737 & 0.009 & $296.0^{*}$ & 0.3 & 2.51 & & & & \\
\hline $12033+2045$ & TDS8195 & 2011.228 & 1 & 0.241 & 0.057 & 60.8 & 6.0 & & & & & \\
\hline $12037+2506$ & A681 & 2011.179 & 1 & 0.321 & 0.013 & 26.5 & 1.6 & & & & & \\
\hline $12037+2506$ & A681 & 2012.000 & 1 & 0.325 & 0.011 & 27.2 & 1.6 & & & & & \\
\hline $12037+2506$ & A681 & 2012.000 & 1 & 0.328 & 0.016 & 27.5 & 3.6 & & & & & \\
\hline
\end{tabular}




\begin{tabular}{|c|c|c|c|c|c|c|c|c|c|c|c|c|}
\hline WDS & Name & Epoch & Bin. & $\begin{array}{c}\rho \\
(")\end{array}$ & $\begin{array}{l}\sigma_{\rho} \\
(")\end{array}$ & $\begin{array}{c}\theta \\
\left({ }^{\circ}\right)\end{array}$ & $\begin{array}{l}\sigma_{\theta} \\
\left(^{\circ}\right)\end{array}$ & $\Delta m$ & Notes & Orbit & $\begin{array}{r}\Delta \rho(\mathrm{O}-\mathrm{C}) \\
(")\end{array}$ & $\begin{array}{r}\Delta \theta(\mathrm{O}-\mathrm{C}) \\
\left({ }^{\circ}\right)\end{array}$ \\
\hline $12037+2506$ & A681 & 2012.350 & 1 & 0.321 & 0.017 & 22.5 & 1.7 & & & & & \\
\hline $12037+2506$ & A681 & 2012.361 & 1 & 0.303 & 0.010 & $24.2^{*}$ & 1.9 & & & & & \\
\hline $12051+4134$ & HDS1703 & 2011.253 & 1 & 0.419 & 0.011 & $326.6^{*}$ & * 0.3 & & & & & \\
\hline $12051+4134$ & HDS1703 & 2012.350 & 1 & 0.438 & 0.007 & 147.3 & 1.7 & & & & & \\
\hline $12052+0824$ & TDS8217 & 2011.272 & 2 & - & - & - & - & & NR & & & \\
\hline $12059+2628$ & HDS1707 & 2011.184 & 1 & 0.293 & 0.051 & 98.6 & 3.3 & & & & & \\
\hline $12060+2312$ & WSI103 & 2012.350 & 1 & - & - & - & - & & NR & & & \\
\hline $12064+1812$ & HDS1708 & 2011.228 & 1 & 0.400 & 0.018 & 122.6 & 2.1 & & & & & \\
\hline $12073+2732$ & TDS8229 & 2011.272 & 2 & - & - & - & - & & NR & & & \\
\hline $12079+0522$ & HEI767 & 2011.231 & 1 & 0.539 & 0.021 & 14.0 & 1.5 & 0.83 & & & & \\
\hline $12079+0522$ & HEI767 & 2011.231 & 1 & 0.577 & 0.015 & 12.6 & 1.5 & & & & & \\
\hline $12080+4242$ & A1998 & 2011.253 & 1 & 0.342 & 0.014 & $349.2^{*}$ & * 2.4 & & & & & \\
\hline $12105+1649$ & CHR135 & 2011.179 & 1 & 0.139 & 0.040 & 125.9 & 8.0 & & & & & \\
\hline $12108+3953$ & STF1606 & 2012.323 & 1 & 0.493 & 0.007 & $151.0^{*}$ & * 0.4 & & & Msn1999a & -0.02 & 1.1 \\
\hline $12149+1947$ & COU178 & 2012.337 & 1 & 1.171 & 0.007 & 319.8 & 0.3 & 2.23 & & & & \\
\hline $12167+4424$ & HDS1729 & 2012.361 & 1 & 0.514 & 0.010 & 36.7 & 0.9 & & & & & \\
\hline $12182+2718$ & HDS1733 & 2012.337 & 1 & 0.312 & 0.007 & 231.8 & 0.3 & 1.44 & & & & \\
\hline $12194+1744$ & A2059 & 2012.279 & 1 & 0.477 & 0.007 & $37.4^{*}$ & 0.7 & & & Lin2017a & 0.01 & 1.2 \\
\hline $12194+1744$ & A2059 & 2012.323 & 1 & 0.469 & 0.007 & $35.7^{*}$ & 0.5 & & & Lin2017a & 0.01 & -0.6 \\
\hline $12194+1744$ & A2059 & 2012.323 & 1 & 0.467 & 0.007 & 36.5 & 0.4 & & & Lin2017a & 0.01 & 0.2 \\
\hline $12195+4456$ & COU1753 & 2012.323 & 1 & 0.697 & 0.017 & 118.1 & 0.6 & & & & & \\
\hline $12223+4826$ & YSC45 & 2012.361 & 1 & 1.046 & 0.007 & $212.9^{*}$ & * 0.3 & & & & & \\
\hline $12232+3257$ & HDS1744 & 2012.350 & 1 & 0.206 & 0.007 & 93.0 & 0.9 & & & & & \\
\hline $12243+2606$ & YSC97 & 2012.350 & 1 & - & - & - & - & & NR & & & \\
\hline $12243+2606$ & YSC97 & 2012.361 & 1 & - & - & - & - & & $\mathrm{NR}$ & & & \\
\hline $12244+4305$ & STT250 & 2012.361 & 1 & 0.291 & 0.007 & $351.7^{*}$ & * 0.4 & & & $\mathrm{PkO} 2018 \mathrm{~b}$ & -0.03 & 0.8 \\
\hline $12288+3155$ & COU965 & 2012.337 & 1 & 0.972 & 0.007 & 250.8 & 0.3 & 2.83 & & & & \\
\hline $12316+3201$ & COU966 & 2012.337 & 1 & 0.196 & 0.040 & 31.8 & 8.0 & 0.28 & & Mnt1999c & 0.06 & 24.3 \\
\hline $12340+2650$ & COU595 & 2012.279 & 1 & 0.953 & 0.007 & $182.0^{*}$ & * 0.3 & & & & & \\
\hline $12351+1719$ & TDS670 & 2012.361 & 1 & 1.159 & 0.007 & $242.6^{*}$ & * 0.5 & & & & & \\
\hline $12351+1719$ & TDS670 & 2012.362 & 1 & 1.179 & 0.007 & 244.2 & 0.3 & 1.85 & & & & \\
\hline $12386+2043$ & HDS1771 & 2012.350 & 1 & 0.328 & 0.007 & 117.5 & 2.3 & & & & & \\
\hline $12397+4444$ & COU1578 & 2011.387 & 1 & 1.463 & 0.007 & $64.3^{*}$ & 0.3 & & & & & \\
\hline $12409+2708$ & COU596 & 2012.337 & 1 & - & - & - & - & & & $\mathrm{NR}$ & & \\
\hline $12422+2622$ & A1851 & 2012.351 & 1 & 0.333 & 0.010 & 9.5 & 0.4 & & & Hei1998 & -0.00 & -2.6 \\
\hline $12430+4008$ & COU1425 & 2011.387 & 1 & 0.849 & 0.007 & $347.8^{*}$ & * 0.3 & & & & & \\
\hline $12430+4008$ & COU1425 & 2012.361 & 1 & 0.850 & 0.007 & $347.8^{*}$ & * 0.3 & & & & & \\
\hline $12441+3546$ & $\mathrm{HO} 256$ & 2011.384 & 1 & 0.578 & 0.007 & $120.6^{*}$ & $* 0.5$ & & & & & \\
\hline $12441+3546$ & HDS1783 & 2012.351 & 1 & - & - & - & - & & NR & & & \\
\hline $12444+2713$ & COU597 & 2012.361 & 1 & 0.249 & 0.009 & 104.0 & 0.7 & & & & & \\
\hline $12461+1715$ & A2061 & 2012.351 & 1 & 1.146 & 0.007 & 14.0 & 0.3 & & & & & \\
\hline $12465+4054$ & HDS1792 & 2012.361 & 1 & - & - & - & - & & NR & & & \\
\hline $12478+4539$ & COU2104 & 2012.361 & 1 & 1.439 & 0.007 & $305.6^{*}$ & * 0.3 & & & & & \\
\hline $12490+3556$ & COU1426 & 2012.337 & 1 & - & - & - & - & & NR & & & \\
\hline $12507+2032$ & HU640 & 2011.384 & 1 & 0.431 & 0.010 & 231.4 & 0.5 & & & Hrt2014b & 0.02 & -0.1 \\
\hline $12507+2032$ & HU640 & 2012.351 & 1 & 0.432 & 0.010 & 234.5 & 0.8 & & & Hrt2014b & 0.02 & -0.4 \\
\hline $12510+3129$ & HDS1804 & 2012.397 & 1 & 0.756 & 0.010 & $258.6^{*}$ & * 0.4 & & & & & \\
\hline $12519+2647$ & HDS1805 & 2012.337 & 1 & 0.421 & 0.027 & 190.8 & 2.3 & 2.39 & & & & \\
\hline $12519+2647$ & HDS1805 & 2012.337 & 1 & 0.399 & 0.027 & 184.1 & 2.3 & 2.85 & & & & \\
\hline $12523+4822$ & YSC100 & 2012.361 & 1 & 0.178 & 0.040 & 148.6 & 8.0 & & & & & \\
\hline $12523+4822$ & YSC100 & 2012.361 & 1 & 0.170 & 0.039 & 149.4 & 1.2 & & & & & \\
\hline $12526+5103$ & COU2189 & 2012.361 & 1 & 0.887 & 0.007 & $101.0^{*}$ & * 0.3 & & & & & \\
\hline $12533+2115$ & STF1687 & 2011.392 & 1 & 1.149 & 0.007 & $194.3^{*}$ & * 0.3 & & & Dru2014 & 0.01 & -0.2 \\
\hline $12533+4246$ & COU1579 & 2011.387 & 1 & 0.223 & 0.007 & $134.2^{*}$ & ${ }^{*} 1.6$ & & & Doc2013a & 0.00 & 3.2 \\
\hline
\end{tabular}




\begin{tabular}{|c|c|c|c|c|c|c|c|c|c|c|c|c|}
\hline WDS & Name & Epoch & Bin. & $\begin{array}{c}\rho \\
(")\end{array}$ & $\begin{array}{l}\sigma_{\rho} \\
(")\end{array}$ & $\begin{array}{c}\theta \\
\left({ }^{\circ}\right)\end{array}$ & $\begin{array}{l}\sigma_{\theta} \\
\left(^{\circ}\right)\end{array}$ & $\Delta m$ & Notes & Orbit & $\begin{array}{r}\Delta \rho(\mathrm{O}-\mathrm{C}) \\
(")\end{array}$ & $\begin{array}{r}\Delta \theta(\mathrm{O}-\mathrm{C}) \\
\left({ }^{\circ}\right)\end{array}$ \\
\hline $12533+4246$ & COU1579 & 2012.361 & 1 & 0.219 & 0.012 & 134.7 & 1.1 & & & Doc2013a & -0.01 & 0.7 \\
\hline $12539+2438$ & YSC47 & 2012.351 & 1 & 0.133 & 0.040 & 337.6 & 8.0 & & & & & \\
\hline $12539+2438$ & YSC47 & 2012.361 & 1 & 0.111 & 0.040 & 324.6 & 8.0 & & & & & \\
\hline $12550+4236$ & COU1580 & 2011.392 & 1 & 0.437 & 0.009 & 107.1 & 0.4 & & & & & \\
\hline $12550+4236$ & COU1580 & 2011.392 & 1 & 0.418 & 0.010 & 107.0 & 1.0 & & & & & \\
\hline $12550+4236$ & COU1580 & 2012.361 & 1 & 0.458 & 0.007 & 106.1 & 0.7 & & & & & \\
\hline $12559+2907$ & TDS682 & 2012.397 & 1 & 1.249 & 0.007 & $265.5^{*}$ & 0.3 & & & & & \\
\hline $12575+2457$ & COU397 & 2012.337 & 1 & 0.707 & 0.007 & 59.9 & 0.3 & 1.62 & & & & \\
\hline $12587+2626$ & COU479 & 2012.351 & 1 & 0.889 & 0.007 & $156.3^{*}$ & 0.8 & & & & & \\
\hline $12591+4709$ & HDS1821 & 2012.397 & 1 & 0.942 & 0.007 & $150.8^{*}$ & 0.5 & & & & & \\
\hline $12595+2814$ & WSI1AB & 2011.395 & 1 & - & - & - & - & & NR & & & \\
\hline $12595+2814$ & WSI1AC & 2011.395 & 1 & 0.700 & 0.009 & 42.0 & 1.1 & & & & & \\
\hline $13012+3852$ & HU1142 & 2012.397 & 1 & - & - & - & - & & $\mathrm{NR}$ & & & \\
\hline $13013+1748$ & COU297 & 2012.337 & 1 & - & - & - & - & & NR & & & \\
\hline $13013+1748$ & COU297 & 2012.361 & 1 & 0.203 & 0.007 & 14.4 & 1.7 & & & & & \\
\hline $13039+3724$ & TDS8673 & 2011.387 & 1 & - & - & - & - & & NR & & & \\
\hline $13049+2438$ & COU298 & 2012.337 & 1 & 0.271 & 0.007 & 8.8 & 0.3 & 1.70 & & & & \\
\hline $13055+3708$ & HDS1830 & 2012.361 & 1 & 0.473 & 0.007 & 164.8 & 0.5 & & & & & \\
\hline $13062+2902$ & BU1083AB & 2011.395 & 1 & - & - & - & - & & NR & & & \\
\hline $13062+2902$ & BU1083AB & 2012.337 & 1 & 0.398 & 0.007 & $66.2^{*}$ & 0.3 & & & & & \\
\hline $13062+2902$ & HJ2638AC & 2012.337 & 1 & 6.337 & 0.032 & $223.1^{*}$ & 0.3 & 5.86 & & & & \\
\hline $13063+2044$ & HU739 & 2012.351 & 1 & 1.486 & 0.007 & $201.7^{*}$ & 0.3 & & & Hrt2014b & 0.01 & 0.2 \\
\hline $13064+2109$ & COU11 & 2012.339 & 1 & 1.749 & 0.009 & 316.7 & 0.3 & 2.73 & & & & \\
\hline $13067+2853$ & HDS1834 & 2011.389 & 1 & 0.728 & 0.007 & 152.1 & 0.4 & & & & & \\
\hline $13068+2657$ & L12 & 2011.389 & 1 & - & - & - & - & & NR & & & \\
\hline $13068+2657$ & L12 & 2012.339 & 1 & 0.235 & 0.007 & 269.9 & 0.3 & 1.49 & & & & \\
\hline $13068+2657$ & L12 & 2012.351 & 1 & - & - & - & - & & NR & & & \\
\hline $13068+4022$ & A2062 & 2011.384 & 1 & 0.659 & 0.007 & 122.2 & 0.4 & & & & & \\
\hline $13081+2657$ & STT260 & 2012.337 & 1 & 0.286 & 0.007 & 32.3 & 0.3 & 0.12 & & Zir2008 & -0.02 & -39.9 \\
\hline $13081+2657$ & STT260 & 2012.337 & 1 & 0.227 & 0.007 & 27.8 & 0.3 & 0.02 & & Zir2008 & -0.08 & -44.4 \\
\hline $13091+2127$ & HU572 & 2012.351 & 1 & 0.469 & 0.007 & 339.7 & 0.3 & & & FMR2012b & -0.03 & 0.4 \\
\hline $13093+4649$ & COU2105 & 2012.359 & 1 & - & - & - & - & & NR & & & \\
\hline $13106+3556$ & ISO13 & 2011.387 & 1 & - & - & - & - & & NR & & & \\
\hline $13112+3050$ & STF1729AB,C & 2012.397 & 2 & 8.521 & 0.043 & 275.1 & 0.3 & 1.75 & & & & \\
\hline $13112+3050$ & A1359 & 2011.384 & 1 & 0.269 & 0.060 & 128.6 & 1.0 & & & & & \\
\hline $13112+3050$ & A1359 & 2012.397 & 1 & 0.335 & 0.070 & 131.8 & 0.3 & 1.53 & & & & \\
\hline $13115+4407$ & COU1910 & 2011.387 & 1 & 0.744 & 0.007 & $34.4^{*}$ & 0.8 & & & & & \\
\hline $13115+4407$ & COU1910 & 2012.359 & 1 & 0.742 & 0.007 & $34.1^{*}$ & 0.3 & & & & & \\
\hline $13118+2931$ & HDS1846 & 2012.361 & 1 & 0.366 & 0.007 & 193.2 & 0.9 & & & & & \\
\hline $13118+2931$ & HDS1846 & 2011.389 & 1 & 0.351 & 0.007 & $193.1^{*}$ & 0.4 & & & & & \\
\hline $13120+4703$ & COU2106AB & 2012.361 & 1 & 0.230 & 0.007 & 92.2 & 0.9 & & & & & \\
\hline $13120+4703$ & COU2106AC & 2012.361 & 1 & 5.567 & 0.028 & 53.5 & 0.3 & & & & & \\
\hline $13121+2415$ & HDS1848 & 2012.351 & 1 & 0.162 & 0.040 & 139.8 & 8.0 & & & & & \\
\hline $13121+2415$ & HDS1848 & 2012.351 & 1 & - & - & - & - & & NR & & & \\
\hline $13128+4030$ & A1606 & 2011.384 & 1 & 1.311 & 0.007 & $196.2^{*}$ & ${ }^{*} 0.3$ & & & & & \\
\hline $13130+4703$ & COU2107 & 2011.389 & 1 & 1.103 & 0.007 & $359.5^{*}$ & 0.6 & & & & & \\
\hline $13131+2838$ & COU400 & 2011.389 & 1 & 0.621 & 0.007 & 108.8 & 0.3 & & & & & \\
\hline $13131+2838$ & COU400 & 2012.339 & 1 & 0.634 & 0.007 & 105.3 & 0.3 & 1.87 & & & & \\
\hline $13137+2949$ & HO55AB & 2012.337 & 1 & - & - & - & - & & NR & & & \\
\hline $13141+3156$ & A1854 & 2012.339 & 1 & 0.190 & 0.040 & 331.5 & 8.0 & 2.02 & & & & \\
\hline $13149+4055$ & COU1911 & 2011.389 & 1 & 0.514 & 0.007 & 87.5 & 0.7 & & & & & \\
\hline $13155+4051$ & CHR180 & 2011.384 & 1 & - & - & - & - & & NR & & & \\
\hline $13155+4051$ & CHR180 & 2011.384 & 1 & 0.627 & 0.007 & $56.7^{*}$ & 0.3 & & & Cve2015b & 0.00 & -0.2 \\
\hline
\end{tabular}




\begin{tabular}{|c|c|c|c|c|c|c|c|c|c|c|c|c|}
\hline WDS & Name & Epoch & Bin. & $\begin{array}{c}\rho \\
(")\end{array}$ & $\begin{array}{l}\sigma_{\rho} \\
(")\end{array}$ & $\begin{array}{c}\theta \\
\left({ }^{\circ}\right)\end{array}$ & $\begin{array}{l}\sigma_{\theta} \\
\left(^{\circ}\right)\end{array}$ & $\Delta m$ & Notes & Orbit & $\begin{array}{r}\Delta \rho(\mathrm{O}-\mathrm{C}) \\
(")\end{array}$ & $\begin{array}{r}\Delta \theta(\mathrm{O}-\mathrm{C}) \\
\left({ }^{\circ}\right)\end{array}$ \\
\hline $13182+4744$ & VBS20 & 2012.397 & 1 & 1.573 & 0.008 & 94.9 & 0.3 & 2.07 & & & & \\
\hline $13183+4736$ & VBS21 & 2012.361 & 1 & 0.883 & 0.007 & 141.5 & 0.7 & & & & & \\
\hline $13188+4806$ & TDS700 & 2011.395 & 1 & 1.295 & 0.012 & $276.2^{*}$ & ${ }^{*} 0.6$ & & & & & \\
\hline $13198+3517$ & HEI520 & 2011.395 & 1 & 1.188 & 0.007 & $65.7^{*}$ & 0.3 & & & & & \\
\hline $13198+4747$ & HU644AB & 2012.359 & 1 & 1.232 & 0.007 & $89.3^{*}$ & 0.3 & & & WSI2015 & 0.01 & -0.4 \\
\hline $13203+1746$ & A2166AB & 2011.384 & 1 & - & - & - & - & & NR & & & \\
\hline $13203+1746$ & A2166AB & 2011.384 & 1 & - & - & - & - & & $\mathrm{NR}$ & & & \\
\hline $13203+1746$ & $\mathrm{~A} 2166 \mathrm{AC}$ & 2011.384 & 1 & 2.170 & 0.011 & $225.8^{*}$ & ${ }^{*} 0.3$ & & $\mathrm{ND}$ & & & \\
\hline $13216+1717$ & COU56AB & 2011.384 & 1 & - & - & - & - & & $\mathrm{NR}$ & & & \\
\hline $13216+1717$ & COU56AB & 2012.337 & 1 & - & - & - & - & & $\mathrm{NR}$ & & & \\
\hline $13216+1717$ & COU56AB & 2012.351 & 1 & - & - & - & - & & NR & & & \\
\hline $13221+3952$ & HDS1876 & 2012.351 & 1 & 0.258 & 0.029 & 89.6 & 1.5 & & & & & \\
\hline $13222+2710$ & A 565 & 2012.359 & 1 & 0.777 & 0.009 & $16.3^{*}$ & 0.3 & & & & & \\
\hline $13225+1543$ & HEI371 & 2012.337 & 1 & 1.658 & 0.008 & 179.4 & 0.3 & & & & & \\
\hline $13225+4242$ & COU1581 & 2012.359 & 1 & 0.316 & 0.007 & $159.4^{*}$ & ${ }^{*} 0.7$ & & & & & \\
\hline $13230+4058$ & COU1582 & 2011.389 & 1 & 0.631 & 0.007 & 80.4 & 0.4 & & & & & \\
\hline $13232+4029$ & POP119 & 2011.384 & 1 & 0.759 & 0.007 & $7.8^{*}$ & 0.5 & & & & & \\
\hline $13232+4822$ & COU1912 & 2011.395 & 1 & 0.409 & 0.013 & $156.8^{*}$ & ${ }^{*} 0.8$ & & & & & \\
\hline $13238+3831$ & HU1259 & 2011.384 & 1 & - & - & - & - & & NR & & & \\
\hline $13238+3831$ & HU1259 & 2012.337 & 1 & - & - & - & - & & $\mathrm{NR}$ & & & \\
\hline $13251+4411$ & ES1548 & 2011.392 & 1 & 1.401 & 0.007 & $3.9^{*}$ & 0.4 & & & & & \\
\hline $13252+3901$ & COU1427 & 2011.389 & 1 & 1.477 & 0.007 & 130.9 & 0.3 & & & & & \\
\hline $13258+4430$ & A1609 & 2012.359 & 1 & 0.430 & 0.007 & $42.2^{*}$ & 0.4 & & & Hei1991 & 0.00 & -0.5 \\
\hline $13258+4430$ & A1609AB,C & 2012.359 & 1 & 2.457 & 0.014 & $219.0^{*}$ & ${ }^{*} 0.3$ & & & & & \\
\hline $13266+3235$ & COU787 & 2012.397 & 1 & 0.315 & 0.007 & 145.1 & 0.3 & 1.91 & & & & \\
\hline $13266+4611$ & COU1913 & 2011.389 & 1 & 0.395 & 0.007 & $159.2^{*}$ & ${ }^{*} 0.6$ & & & & & \\
\hline $13276+2116$ & GII60AC & 2012.361 & 1 & 2.881 & 0.014 & 127.8 & 0.3 & & $\mathrm{ND}$ & & & \\
\hline $13276+2116$ & GII60AC & 2012.362 & 1 & 2.897 & 0.014 & 127.9 & 0.3 & 3.83 & $\mathrm{ND}$ & & & \\
\hline $13276+2116$ & GII60AC & 2012.397 & 1 & 2.896 & 0.014 & 126.9 & 0.3 & 4.22 & $\mathrm{ND}$ & & & \\
\hline $13276+2116$ & TOK46Aa,Ab & 2012.361 & 1 & 0.168 & 0.040 & 178.5 & 8.0 & & & & & \\
\hline $13281+4447$ & TDS8836 & 2011.387 & 1 & - & - & - & - & & NR & & & \\
\hline $13282+5035$ & YSC48 & 2012.361 & 1 & - & - & - & - & & NR & & & \\
\hline $13284+3643$ & COU1428 & 2012.337 & 1 & 0.348 & 0.007 & 70.8 & 0.3 & 2.78 & & & & \\
\hline $13284+3643$ & COU1428 & 2012.337 & 1 & 0.352 & 0.022 & 67.9 & 0.6 & & & & & \\
\hline $13284+3643$ & COU1428 & 2012.339 & 1 & 0.368 & 0.007 & 68.7 & 0.3 & 2.71 & & & & \\
\hline $13290+2913$ & COU598 & 2011.389 & 1 & 1.702 & 0.009 & $356.7^{*}$ & ${ }^{*} 0.3$ & & & & & \\
\hline $13290+4751$ & YSC103 & 2012.397 & 1 & 0.190 & 0.040 & 0.0 & 8.0 & 1.17 & & & & \\
\hline $13292+4926$ & HDS1888 & 2011.395 & 1 & - & - & - & - & & $\mathrm{NR}$ & & & \\
\hline $13293+4847$ & COU1914 & 2011.392 & 1 & 0.492 & 0.007 & $184.5^{*}$ & ${ }^{*} 0.3$ & & & & & \\
\hline $13305+3430$ & A1857 & 2011.387 & 1 & 0.475 & 0.010 & $343.4^{*}$ & ${ }^{*} 0.6$ & & & & & \\
\hline $13318+2917$ & BEU17 & 2012.359 & 1 & - & - & - & - & & NR & & & \\
\hline $13320+3108$ & WOR24 & 2011.389 & 1 & 0.295 & 0.007 & 70.0 & 0.9 & & & Doc $2000 \mathrm{~b}$ & -0.01 & 1.3 \\
\hline $13320+3108$ & WOR24 & 2012.337 & 1 & 0.322 & 0.007 & 62.9 & 0.3 & & & Doc2000b & -0.01 & 2.2 \\
\hline $13329+3454$ & STT269 & 2012.339 & 1 & 0.267 & 0.007 & 225.4 & 0.3 & 0.68 & & Hei1997 & -0.04 & 1.8 \\
\hline $13331+4316$ & COU1754 & 2011.387 & 1 & - & - & - & - & & NR & & & \\
\hline $13331+4316$ & COU1754 & 2012.359 & 1 & - & - & - & - & & $\mathrm{NR}$ & & & \\
\hline $13333+4526$ & A1610 & 2012.397 & 1 & 0.919 & 0.007 & 267.7 & 0.3 & 1.19 & & & & \\
\hline $13336+2944$ & A1095 & 2012.361 & 1 & 0.340 & 0.007 & 283.9 & 0.5 & & & Ole2002a & -0.01 & -0.1 \\
\hline $13344+3559$ & HDS1906 & 2011.384 & 1 & 0.183 & 0.040 & $22.0^{*}$ & 8.0 & & & & & \\
\hline $13348+4242$ & HDS1908 & 2011.387 & 1 & 0.462 & 0.015 & $29.6^{*}$ & 1.1 & & & & & \\
\hline $13357+3935$ & COU1583 & 2011.384 & 1 & 1.049 & 0.007 & $60.7^{*}$ & 0.4 & & & & & \\
\hline $13360+2123$ & HU741 & 2012.339 & 1 & 0.239 & 0.007 & 1.5 & 0.3 & 1.25 & & & & \\
\hline $13360+2123$ & HU741 & 2011.389 & 1 & 0.255 & 0.007 & $0.9^{*}$ & 0.5 & & & & & \\
\hline
\end{tabular}




\begin{tabular}{|c|c|c|c|c|c|c|c|c|c|c|c|c|}
\hline WDS & Name & Epoch & Bin. & $\begin{array}{c}\rho \\
(")\end{array}$ & $\begin{array}{l}\sigma_{\rho} \\
(")\end{array}$ & $\begin{array}{c}\theta \\
\left({ }^{\circ}\right)\end{array}$ & $\begin{array}{l}\sigma_{\theta} \\
\left(^{\circ}\right)\end{array}$ & $\Delta m$ & Notes & Orbit & $\begin{array}{r}\Delta \rho(\mathrm{O}-\mathrm{C}) \\
(")\end{array}$ & $\begin{array}{r}\Delta \theta(\mathrm{O}-\mathrm{C}) \\
\left({ }^{\circ}\right)\end{array}$ \\
\hline $13377+5043$ & STF1770 & 2011.392 & 1 & 1.700 & 0.008 & $122.2^{*}$ & ${ }^{*} 0.3$ & & & & & \\
\hline $13377+5043$ & STF 1770 & 2011.392 & 1 & 1.694 & 0.008 & 122.1 & 0.3 & & & & & \\
\hline $13379+4808$ & ES608 & 2012.361 & 1 & 1.692 & 0.011 & $331.3^{*}$ & ${ }^{k} 0.3$ & & & USN2002 & -0.00 & 2.0 \\
\hline $13387+3823$ & COU1429 & 2011.384 & 1 & 0.679 & 0.007 & $65.1^{*}$ & 0.3 & & & & & \\
\hline $13387+4126$ & COU1584 & 2012.359 & 1 & - & - & - & - & & NR & & & \\
\hline $13389+2127$ & HU645 & 2011.389 & 1 & 0.785 & 0.007 & 24.5 & 0.5 & & & & & \\
\hline $13398+3625$ & COU1264 & 2011.384 & 1 & 0.377 & 0.017 & $257.7^{*}$ & ${ }^{*} 0.9$ & & & & & \\
\hline $13398+3625$ & COU1264 & 2012.397 & 1 & 0.430 & 0.017 & 268.2 & 0.3 & 3.05 & & & & \\
\hline $13400+1235$ & HDS1917 & 2011.387 & 1 & 0.173 & 0.040 & 93.9 & 8.0 & & & & & \\
\hline $13411+3719$ & TDS718 & 2011.395 & 1 & 1.711 & 0.009 & 42.6 & 0.3 & & & & & \\
\hline $13419+1407$ & HDS1925 & 2011.387 & 1 & 0.260 & 0.049 & $208.6^{*}$ & ${ }^{*} 2.9$ & & & & & \\
\hline $13419+4725$ & HDS1924 & 2011.392 & 1 & 0.751 & 0.015 & $310.2^{*}$ & ${ }^{k} 0.8$ & & & & & \\
\hline $13433+4023$ & COU1431 & 2012.397 & 1 & - & - & - & - & & $\mathrm{NR}$ & & & \\
\hline $13433+4023$ & COU1431 & 2011.384 & 1 & 1.137 & 0.007 & $343.3^{*}$ & ${ }^{k} 0.3$ & & & & & \\
\hline $13446+3224$ & COU788 & 2011.384 & 1 & 1.689 & 0.008 & $298.4^{*}$ & ${ }^{k} 0.3$ & & & & & \\
\hline $13446+4819$ & TDS8951 & 2011.395 & 1 & - & - & - & - & & NR & & & \\
\hline $13446+4819$ & TDS8951 & 2011.395 & 1 & 0.673 & 0.048 & $139.5^{*}$ & ${ }^{k} 0.7$ & & & & & \\
\hline $13453+4559$ & COU1915 & 2011.389 & 1 & 1.610 & 0.008 & $75.8^{*}$ & 0.3 & & & & & \\
\hline $13457+2231$ & YSC49 & 2012.339 & 1 & 0.149 & 0.040 & 134.1 & 8.0 & 0.00 & & & & \\
\hline $13457+2231$ & YSC49 & 2012.359 & 1 & 0.171 & 0.040 & 134.4 & 8.0 & & & & & \\
\hline $13477+2128$ & HDS1939 & 2011.389 & 1 & 1.284 & 0.024 & 149.7 & 0.3 & & & & & \\
\hline $13477+2128$ & HDS1939 & 2011.389 & 1 & 1.271 & 0.010 & $149.9^{*}$ & ${ }^{k} 0.5$ & & & & & \\
\hline $13482+2248$ & COU401 & 2011.384 & 1 & - & - & - & - & & NR & & & \\
\hline $13488+2753$ & HDS1942AC & 2011.395 & 1 & 1.744 & 0.014 & 42.7 & 0.3 & & & & & \\
\hline $13493+3140$ & COU601 & 2012.339 & 1 & 1.327 & 0.007 & 301.0 & 0.3 & 2.76 & & & & \\
\hline $13509+3555$ & COU1132 & 2012.339 & 1 & 0.796 & 0.007 & 147.1 & 0.3 & 3.06 & & & & \\
\hline $13509+4531$ & HDS1949 & 2011.395 & 1 & 0.134 & 0.040 & 45.0 & 8.0 & & & & & \\
\hline $13512+2948$ & A685 & 2012.397 & 1 & 0.790 & 0.007 & 19.2 & 0.3 & 2.44 & & & & \\
\hline $13514+2620$ & SKF260AB & 2012.339 & 1 & 3.213 & 0.016 & 1.3 & 0.3 & 4.20 & & & & \\
\hline $13514+2620$ & YSC50Aa,Ab & 2012.339 & 1 & 0.221 & 0.007 & 218.9 & 0.3 & 1.27 & & & & \\
\hline $13518+3649$ & COU1133 & 2012.351 & 1 & 0.270 & 0.014 & 156.4 & 1.7 & & & & & \\
\hline $13524+3851$ & TDS9012 & 2011.395 & 1 & 0.632 & 0.007 & 101.0 & 0.5 & & & & & \\
\hline $13535+3618$ & COU1134 & 2011.384 & 1 & 1.559 & 0.008 & $250.6^{*}$ & ${ }^{k} 0.3$ & & & & & \\
\hline $13554+5002$ & COU2689 & 2012.359 & 1 & 0.649 & 0.007 & $327.4^{*}$ & ${ }^{*} 0.5$ & & & & & \\
\hline $13583+3922$ & COU1432 & 2012.361 & 1 & 1.670 & 0.008 & $192.0^{*}$ & ${ }^{k} 0.3$ & & & & & \\
\hline $13584+1120$ & HDS1959 & 2011.387 & 1 & 0.235 & 0.007 & 180.1 & 0.5 & & & & & \\
\hline $13586+3741$ & TDS9051 & 2011.395 & 1 & - & - & - & - & & NR & & & \\
\hline $13591+3549$ & COU1265 & 2011.384 & 1 & 1.715 & 0.009 & $93.9^{*}$ & 0.3 & & & & & \\
\hline $13593+3042$ & COU602 & 2012.339 & 1 & 0.435 & 0.007 & 124.8 & 0.6 & & & & & \\
\hline $13593+3042$ & COU602 & 2012.339 & 1 & 0.388 & 0.007 & 124.7 & 0.3 & 1.79 & & & & \\
\hline $14001+3827$ & TDS9064 & 2011.395 & 1 & - & - & - & - & & NR & & & \\
\hline $14012+2522$ & A569 & 2012.359 & 1 & 0.597 & 0.007 & $147.4^{*}$ & ${ }^{k} 0.3$ & & & & & \\
\hline $14014+1710$ & COU2193 & 2012.359 & 1 & 2.198 & 0.011 & 315.8 & 0.3 & 3.14 & & & & \\
\hline $14017+3759$ & COU1433 & 2012.339 & 1 & 0.494 & 0.007 & 296.7 & 0.3 & 2.30 & & & & \\
\hline $14034+2409$ & COU302 & 2012.359 & 1 & 0.861 & 0.007 & 175.3 & 0.3 & 3.20 & & & & \\
\hline $14029+2434$ & COU301 & 2011.384 & 1 & 0.906 & 0.015 & $126.1^{*}$ & ${ }^{*} 0.6$ & & & & & \\
\hline $14029+2434$ & COU301 & 2011.384 & 1 & 0.906 & 0.007 & 124.4 & 0.3 & 3.69 & & & & \\
\hline $14029+2434$ & COU301 & 2011.384 & 1 & 0.915 & 0.007 & $127.2^{*}$ & ${ }^{*} 0.5$ & & & & & \\
\hline $14029+2434$ & COU301 & 2012.339 & 1 & 0.897 & 0.007 & 126.4 & 0.3 & 3.38 & & & & \\
\hline $14029+2434$ & COU301 & 2012.339 & 1 & 0.901 & 0.007 & 125.2 & 0.3 & 3.38 & & & & \\
\hline $14048+3243$ & COU603 & 2011.389 & 1 & 0.697 & 0.007 & $53.4^{*}$ & 0.3 & & & & & \\
\hline $14059+3848$ & HU1263 & 2011.387 & 1 & 0.275 & 0.010 & $195.9^{*}$ & ${ }^{*} 1.3$ & & & & & \\
\hline $14059+4059$ & COU1585 & 2011.389 & 1 & 0.899 & 0.007 & $214.6^{*}$ & ${ }^{k} 0.3$ & & & & & \\
\hline
\end{tabular}




\begin{tabular}{|c|c|c|c|c|c|c|c|c|c|c|c|c|}
\hline WDS & Name & Epoch & Bin. & $\begin{array}{c}\rho \\
(")\end{array}$ & $\begin{array}{l}\sigma_{\rho} \\
(")\end{array}$ & $\begin{array}{c}\theta \\
\left({ }^{\circ}\right)\end{array}$ & $\begin{array}{l}\sigma_{\theta} \\
\left(^{\circ}\right)\end{array}$ & $\Delta m$ & Notes & Orbit & $\begin{array}{r}\Delta \rho(\mathrm{O}-\mathrm{C}) \\
(")\end{array}$ & $\begin{array}{r}\Delta \theta(\mathrm{O}-\mathrm{C}) \\
\left({ }^{\circ}\right)\end{array}$ \\
\hline $14066+2443$ & COU303 & 2012.359 & 1 & 1.930 & 0.010 & 288.9 & 0.3 & 3.51 & & & & \\
\hline $14073+2505$ & A2384 & 2011.387 & 1 & 1.046 & 0.007 & $111.8^{*}$ & 0.3 & & & & & \\
\hline $14081+4503$ & COU1755 & 2011.392 & 1 & 1.162 & 0.007 & $163.4^{*}$ & 0.3 & & & & & \\
\hline $14081+4503$ & COU1755 & 2011.392 & 1 & 1.158 & 0.007 & $163.6^{*}$ & 0.3 & & & & & \\
\hline $14082+3614$ & STT276AB & 2012.339 & 1 & - & - & - & - & & NR & & & \\
\hline $14084+2910$ & A1363 & 2011.387 & 1 & 2.577 & 0.013 & $229.4^{*}$ & 0.3 & & & & & \\
\hline $14087+3341$ & HU742 & 2012.339 & 1 & 0.209 & 0.007 & 341.7 & 0.3 & 0.97 & & FMR2012h & -0.08 & -2.3 \\
\hline $14087+3341$ & HU742 & 2011.395 & 1 & 0.237 & 0.010 & $349.4^{*}$ & 1.5 & & & FMR2012h & -0.03 & 2.4 \\
\hline $14105+4823$ & COU2108 & 2012.359 & 1 & 0.542 & 0.027 & 324.6 & 0.3 & 2.12 & & & & \\
\hline $14105+4823$ & COU2108 & 2012.397 & 1 & 0.563 & 0.027 & 321.8 & 0.3 & 1.99 & & & & \\
\hline $14109+1513$ & HDS1989Aa,Ab & 2011.387 & 1 & 0.459 & 0.020 & $353.2^{*}$ & 1.8 & & & & & \\
\hline $14111+3628$ & GII96Aa,Ab & 2011.387 & 1 & 0.356 & 0.007 & 192.4 & 0.6 & & $\mathrm{ND}$ & & & \\
\hline $14111+3628$ & HU1264AB & 2011.387 & 1 & 1.356 & 0.007 & $359.9^{*}$ & 0.3 & & & & & \\
\hline $14113+3013$ & COU605 & 2012.359 & 1 & 0.249 & 0.007 & $340.2^{*}$ & 0.7 & & & & & \\
\hline $14122+2722$ & $\mathrm{~J} 1125$ & 2011.395 & 1 & 0.691 & 0.007 & $95.7^{*}$ & 0.5 & & & & & \\
\hline $14122+4411$ & STT278 & 2011.392 & 1 & - & - & - & - & & NR & & & \\
\hline $14122+4411$ & STT278 & 2012.359 & 1 & 0.361 & 0.007 & $277.0^{*}$ & 0.5 & & & Hrt2009 & -0.02 & -1.1 \\
\hline $14127+2233$ & HDS1993 & 2011.387 & 1 & 0.164 & 0.040 & 148.9 & 8.0 & & & & & \\
\hline $14127+2233$ & HDS1993 & 2011.395 & 1 & 0.176 & 0.040 & 145.6 & 8.0 & & & & & \\
\hline $14127+2349$ & YSC53 & 2012.359 & 1 & 1.123 & 0.007 & $129.5^{*}$ & 0.3 & & & & & \\
\hline $14129+5043$ & YSC105 & 2012.361 & 1 & 0.595 & 0.010 & 31.5 & 0.5 & & & & & \\
\hline $14129+5043$ & YSC105 & 2012.361 & 1 & 0.585 & 0.007 & 28.4 & 0.4 & & & & & \\
\hline $14129+5043$ & YSC105 & 2012.361 & 1 & 0.593 & 0.007 & 209.4 & 0.3 & 3.76 & & & & \\
\hline $14138+1720$ & HDS1998AB & 2012.359 & 1 & 0.891 & 0.007 & $358.9^{*}$ & 0.3 & & & & & \\
\hline $14138+1720$ & YSC54 & 2012.359 & 1 & 0.201 & 0.007 & 172.6 & 0.4 & & & & & \\
\hline $14138+3059$ & COU606 & 2011.387 & 1 & 0.145 & 0.040 & 220.5 & 8.0 & & & Doc2008a & 0.02 & 9.6 \\
\hline $14138+3059$ & COU606 & 2012.339 & 1 & 0.082 & 0.007 & 181.8 & 0.3 & 1.93 & & Doc2008a & -0.04 & -5.0 \\
\hline $14140+3720$ & COU1434 & 2012.361 & 1 & 0.541 & 0.014 & 105.9 & 1.0 & & & & & \\
\hline $14164+3708$ & HDS2005AB & 2011.389 & 1 & 0.161 & 0.040 & 171.2 & 8.0 & & & & & \\
\hline $14164+3708$ & HDS2005AC & 2011.384 & 1 & 3.565 & 0.018 & $174.8^{*}$ & 0.3 & & & & & \\
\hline $14165+3334$ & HU1266 & 2011.392 & 1 & 0.218 & 0.047 & 144.4 & 6.1 & & & Doc2012e & -0.03 & -1.3 \\
\hline $14165+3334$ & HU1266 & 2011.392 & 1 & 0.215 & 0.037 & 146.9 & 5.4 & & & Doc2012e & -0.04 & 1.2 \\
\hline $14165+3334$ & HU1266 & 2012.397 & 1 & 0.177 & 0.040 & 146.5 & 8.0 & 1.27 & & Doc2012e & -0.07 & -2.0 \\
\hline $14167+4055$ & COU1756 & 2011.390 & 1 & 1.021 & 0.007 & $42.4^{*}$ & 0.3 & & & & & \\
\hline $14185+4755$ & BU1273 & 2011.395 & 1 & 1.305 & 0.007 & $176.8^{*}$ & 0.3 & & & & & \\
\hline $14192+3755$ & COU1435 & 2011.390 & 1 & 1.688 & 0.030 & 42.1 & 0.9 & & & & & \\
\hline $14203+4830$ & STF1834 & 2011.395 & 1 & 1.618 & 0.008 & $103.2^{*}$ & 0.3 & & & Izm2019 & 0.01 & 0.3 \\
\hline $14205+2634$ & DAN1 & 2012.361 & 1 & - & - & - & - & & NR & & & \\
\hline $14205+2634$ & DAN1 & 2012.361 & 1 & 0.147 & 0.040 & 131.2 & 8.0 & & & & & \\
\hline $14208+4617$ & COU2194 & 2012.362 & 1 & 0.233 & 0.007 & 56.7 & 1.3 & & & & & \\
\hline $14208+4617$ & COU2194 & 2011.395 & 1 & 0.173 & 0.040 & 52.5 & 8.0 & & & & & \\
\hline $14213+3050$ & COU482 & 2012.397 & 1 & 0.655 & 0.007 & 121.0 & 0.3 & 1.02 & & & & \\
\hline $14224+3557$ & TDS9175 & 2011.395 & 1 & 0.232 & 0.007 & 6.5 & 2.6 & & & & & \\
\hline $14233+2111$ & COU61 & 2011.392 & 1 & 1.914 & 0.010 & 100.2 & 0.3 & & & & & \\
\hline $14234+4736$ & A149 & 2012.397 & 1 & 0.780 & 0.007 & 123.7 & 0.3 & 1.18 & & & & \\
\hline $14249+3730$ & HDS2029 & 2011.384 & 1 & 0.176 & 0.040 & $284.1^{*}$ & 8.0 & & & & & \\
\hline $14260+4213$ & COU1757 & 2011.387 & 1 & 0.114 & 0.040 & 54.7 & 8.0 & & & Hrt2014b & -0.01 & 5.3 \\
\hline $14260+4213$ & COU1757 & 2012.359 & 1 & 0.134 & 0.040 & 73.5 & 8.0 & & & Hrt2014b & 0.01 & 12.6 \\
\hline $14260+4213$ & COU1757 & 2012.362 & 1 & 0.087 & 0.040 & 71.0 & 8.0 & & & Hrt2014b & -0.04 & 10.1 \\
\hline $14262+2154$ & HDS2032 & 2011.392 & 1 & 0.148 & 0.040 & 170.1 & 8.0 & & & & & \\
\hline $14277+2842$ & TDS750 & 2011.384 & 1 & 1.049 & 0.007 & $299.9^{*}$ & 0.4 & & & & & \\
\hline $14280+3546$ & COU1266 & 2011.387 & 1 & 1.221 & 0.007 & $56.7^{*}$ & 0.3 & & & & & \\
\hline $14280+3546$ & COU1266 & 2012.362 & 1 & 1.215 & 0.007 & $237.3^{*}$ & 0.3 & & & & & \\
\hline
\end{tabular}




\begin{tabular}{|c|c|c|c|c|c|c|c|c|c|c|c|c|}
\hline WDS & Name & Epoch & Bin. & $\begin{array}{c}\rho \\
(")\end{array}$ & $\begin{array}{l}\sigma_{\rho} \\
(")\end{array}$ & $\begin{array}{c}\theta \\
\left(^{\circ}\right)\end{array}$ & $\begin{array}{l}\sigma_{\theta} \\
\left({ }^{\circ}\right)\end{array}$ & $\Delta m$ & Notes & Orbit & $\begin{array}{r}\Delta \rho(\mathrm{O}-\mathrm{C}) \\
(")\end{array}$ & $\begin{array}{r}\Delta \theta(\mathrm{O}-\mathrm{C}) \\
\left({ }^{\circ}\right)\end{array}$ \\
\hline $14286+1818$ & COU2508 & 2012.339 & 1 & 0.440 & 0.007 & 253.5 & 0.3 & 2.25 & & & & \\
\hline $14289+4226$ & COU1758 & 2011.390 & 1 & 0.582 & 0.007 & $147.9^{*}$ & ${ }^{*} 0.4$ & & & & & \\
\hline $14293+1318$ & HDS2042 & 2011.387 & 1 & 0.271 & 0.024 & $123.5^{*}$ & ${ }^{*} 5.2$ & & & & & \\
\hline $14293+1318$ & HDS2042 & 2011.387 & 1 & 0.222 & 0.028 & 132.9 & 5.1 & & & & & \\
\hline $14295+3612$ & HU1268 & 2011.395 & 1 & 0.281 & 0.010 & $341.1^{*}$ & ${ }^{*} 1.3$ & & & Hrt2008 & -0.01 & 2.1 \\
\hline $14295+3612$ & HU1268 & 2012.359 & 1 & 0.298 & 0.017 & $341.2^{*}$ & ${ }^{*} 1.4$ & & & Hrt2008 & 0.00 & 3.2 \\
\hline $14295+3759$ & COU1436 & 2011.384 & 1 & 0.946 & 0.007 & $42.8^{*}$ & 0.3 & & & & & \\
\hline $14298+1550$ & HEI532BC & 2012.339 & 2 & 1.742 & 0.015 & 69.1 & 0.3 & 3.14 & & & & \\
\hline $14301+4737$ & COU2387 & 2011.390 & 1 & 0.339 & 0.007 & $343.3^{*}$ & ${ }^{*} 0.4$ & & & & & \\
\hline $14303+4709$ & COU1917 & 2012.362 & 1 & 0.413 & 0.007 & 38.5 & 0.5 & & & & & \\
\hline $14305+2055$ & COU97 & 2012.359 & 1 & 0.296 & 0.010 & $251.1^{*}$ & ${ }^{*} 2.0$ & & & & & \\
\hline $14311+2801$ & COU404 & 2011.384 & 1 & 1.316 & 0.007 & $176.9^{*}$ & 0.3 & & & & & \\
\hline $14312+3426$ & TDS9227 & 2011.395 & 1 & 1.841 & 0.009 & $172.8^{*}$ & 0.3 & & & & & \\
\hline $14317+3554$ & COU1267 & 2011.390 & 1 & 0.734 & 0.007 & 62.5 & 0.3 & & & & & \\
\hline $14323+2641$ & A 570 & 2011.392 & 1 & 0.191 & 0.040 & 9.8 & 8.0 & & & Hei1991 & 0.02 & 3.0 \\
\hline $14323+2641$ & A 570 & 2012.474 & 1 & 0.193 & 0.040 & $172.6^{*}$ & 8.0 & & & Hei1991 & 0.03 & $2.5^{Q}$ \\
\hline $14325+4911$ & HU57BC & 2012.362 & 1 & 1.102 & 0.007 & $134.7^{*}$ & ${ }^{*} 0.3$ & 0.57 & & & & \\
\hline $14337+4300$ & COU1586 & 2011.392 & 1 & 1.782 & 0.009 & $142.7^{*}$ & 0.3 & & & & & \\
\hline $14340+4113$ & COU1437 & 2012.359 & 1 & 0.181 & 0.040 & 175.2 & 8.0 & & & & & \\
\hline $14340+4500$ & COU1587 & 2011.392 & 1 & 0.756 & 0.007 & $25.5^{*}$ & 0.4 & & & & & \\
\hline $14345+3801$ & HDS2057 & 2011.387 & 1 & 0.792 & 0.010 & $294.2^{*}$ & ${ }^{*} 0.5$ & & & & & \\
\hline $14353+4302$ & YSC55 & 2012.359 & 1 & 0.530 & 0.021 & 128.2 & 0.6 & & & & & \\
\hline $14367+2014$ & COU98 & 2012.397 & 1 & 0.318 & 0.007 & 168.2 & 0.3 & 2.60 & & & & \\
\hline $14376+3137$ & HDS2062 & 2012.362 & 1 & 0.139 & 0.040 & 149.6 & 8.0 & & & & & \\
\hline $14376+3137$ & HDS2062 & 2011.395 & 1 & 0.128 & 0.040 & 147.0 & 8.0 & & & & & \\
\hline $14393+2648$ & A571 & 2011.384 & 1 & 1.816 & 0.009 & $108.6^{*}$ & $* 0.3$ & & & & & \\
\hline $14403+3331$ & COU789 & 2011.390 & 1 & 0.992 & 0.009 & $217.7^{*}$ & * 0.5 & & & & & \\
\hline $14407+3117$ & STF1867 & 2011.461 & 1 & 0.700 & 0.008 & $355.7^{*}$ & * 0.3 & & & Zir2013a & 0.02 & 0.9 \\
\hline $14426+3842$ & COU1268 & 2011.384 & 1 & 0.193 & 0.040 & 131.6 & 8.0 & & & & & \\
\hline $14426+3842$ & COU1268 & 2012.362 & 1 & 0.199 & 0.040 & 143.9 & 8.0 & & & & & \\
\hline $14455+4223$ & STT285 & 2011.392 & 1 & 0.506 & 0.007 & $88.0^{*}$ & 0.4 & & & Sca2015b & 0.01 & 0.9 \\
\hline $14459+2344$ & COU100 & 2011.390 & 1 & - & - & - & - & & NR & & & \\
\hline $14459+2344$ & COU100 & 2012.359 & 1 & - & - & - & - & & NR & & & \\
\hline $14460+4723$ & HDS2079 & 2011.390 & 1 & 0.631 & 0.007 & $313.8^{*}$ & $* 0.7$ & & & & & \\
\hline $14463+3146$ & HDS2080 & 2011.387 & 1 & 0.125 & 0.040 & 129.3 & 8.0 & & & & & \\
\hline $14477+2406$ & HO263 & 2011.395 & 1 & - & - & - & - & & NR & & & \\
\hline $14491+4058$ & HDS2088 & 2011.387 & 1 & 1.320 & 0.007 & $89.3^{*}$ & 0.3 & & & & & \\
\hline $14492+4814$ & HU647 & 2011.392 & 1 & 0.165 & 0.083 & 81.4 & 8.0 & & & & & \\
\hline $14492+4814$ & HU647 & 2011.392 & 1 & 0.201 & 0.019 & 79.5 & 2.7 & & & & & \\
\hline $14493+4950$ & HDS2091 & 2011.392 & 1 & 1.061 & 0.007 & $345.1^{*}$ & * 0.3 & & & & & \\
\hline $14506+4326$ & HDS2094 & 2011.392 & 1 & 0.208 & 0.009 & $22.4^{*}$ & 1.0 & & & & & \\
\hline $14515+4456$ & STT287 & 2011.395 & 1 & 0.691 & 0.007 & $0.4^{*}$ & 0.3 & & & Hei1997 & -0.07 & 1.8 \\
\hline $14521+2017$ & HO389 & 2011.458 & 1 & 1.512 & 0.008 & $86.6^{*}$ & 0.3 & & & & & \\
\hline $14521+4629$ & HDS2097 & 2011.392 & 1 & 0.469 & 0.007 & $45.1^{*}$ & 2.7 & & & & & \\
\hline $14521+4629$ & HDS2097 & 2011.392 & 1 & 0.491 & 0.026 & 33.0 & 8.2 & & & & & \\
\hline $14523+4413$ & COU1588 & 2011.395 & 1 & 1.044 & 0.007 & $203.3^{*}$ & * 0.3 & & & & & \\
\hline $14534+1542$ & STT288 & 2011.461 & 1 & 1.075 & 0.007 & $160.8^{*}$ & * 0.3 & & & Hei1998 & 0.02 & 0.2 \\
\hline $14546+2252$ & COU408 & 2011.392 & 1 & 1.874 & 0.009 & $354.5^{*}$ & * 0.3 & & & & & \\
\hline $14558+3939$ & A1627 & 2012.359 & 1 & 0.216 & 0.007 & 191.5 & 1.1 & & & Baz1993a & -0.00 & 1.0 \\
\hline $14558+3939$ & A1627 & 2011.384 & 1 & 0.159 & 0.040 & 190.9 & 8.0 & & & Baz1993a & -0.06 & 2.3 \\
\hline $14560+3807$ & TDS9348 & 2011.395 & 1 & 1.679 & 0.008 & $258.8^{*}$ & * 0.3 & & & & & \\
\hline $14565+1706$ & YSC7 & 2012.359 & 1 & 0.318 & 0.015 & 166.5 & 2.4 & & & & & \\
\hline
\end{tabular}




\begin{tabular}{|c|c|c|c|c|c|c|c|c|c|c|c|c|}
\hline WDS & Name & Epoch & Bin. & $\begin{array}{c}\rho \\
(")\end{array}$ & $\begin{array}{l}\sigma_{\rho} \\
(")\end{array}$ & $\begin{array}{c}\theta \\
\left(^{\circ}\right)\end{array}$ & $\begin{array}{l}\sigma_{\theta} \\
\left({ }^{\circ}\right)\end{array}$ & $\Delta m$ & Notes & Orbit & $\begin{array}{r}\Delta \rho(\mathrm{O}-\mathrm{C}) \\
(")\end{array}$ & $\begin{array}{r}\Delta \theta(\mathrm{O}-\mathrm{C}) \\
\left({ }^{\circ}\right)\end{array}$ \\
\hline $14569+2034$ & HU648 & 2011.387 & 1 & 1.453 & 0.007 & $135.8^{*}$ & ${ }^{*} 0.3$ & & & & & \\
\hline $14569+3429$ & HDS2110 & 2011.384 & 1 & 1.399 & 0.008 & $22.5^{*}$ & 0.3 & & & & & \\
\hline $14575+3124$ & HDS2112 & 2011.392 & 1 & 0.814 & 0.007 & 156.8 & 0.4 & & & & & \\
\hline $14588+3551$ & COU1136 & 2012.359 & 1 & 0.143 & 0.041 & 148.4 & 8.0 & & & Gur2020 & 0.10 & -11.3 \\
\hline $14593+4649$ & COU1760 & 2011.395 & 1 & 0.196 & 0.040 & $251.6^{*}$ & 8.0 & & & Hrt2014b & -0.03 & 2.5 \\
\hline $14593+4649$ & COU1760 & 2011.395 & 1 & 0.249 & 0.027 & 252.8 & 1.1 & & & Hrt2014b & 0.03 & 3.8 \\
\hline $14595+1753$ & COU188 & 2012.474 & 1 & 0.282 & 0.034 & 51.0 & 6.5 & & & & & \\
\hline $14595+3957$ & COU1269 & 2011.384 & 1 & 0.154 & 0.040 & 110.1 & 8.0 & & & & & \\
\hline $14595+3957$ & COU1269 & 2011.395 & 1 & 0.202 & 0.028 & 103.1 & 2.5 & & & & & \\
\hline $14595+3957$ & COU1269 & 2012.359 & 1 & - & - & - & - & & NR & & & \\
\hline $14597+3410$ & A1365 & 2011.461 & 1 & 2.001 & 0.010 & $58.6^{*}$ & 0.3 & & & & & \\
\hline $15005+3741$ & COU1270 & 2011.384 & 1 & 0.449 & 0.007 & $227.8^{*}$ & 0.4 & & & & & \\
\hline $15009+4526$ & HDS2118 & 2011.390 & 1 & 1.869 & 0.009 & $169.6^{*}$ & 0.3 & & & & & \\
\hline $15010+4543$ & HDS2119 & 2011.390 & 1 & 0.401 & 0.013 & $122.6^{*}$ & 3.1 & & & & & \\
\hline $15029+1655$ & COU63 & 2011.387 & 1 & 1.953 & 0.010 & $46.8^{*}$ & 0.3 & & & & & \\
\hline $15034+3319$ & COU791 & 2011.390 & 1 & 1.241 & 0.007 & $17.4^{*}$ & 0.3 & & & & & \\
\hline $15038+2006$ & HU744 & 2011.392 & 1 & 0.966 & 0.011 & $353.4^{*}$ & 0.6 & & & & & \\
\hline $15038+4739$ & STF1909 & 2011.458 & 1 & 1.471 & 0.007 & $61.4^{*}$ & 0.3 & & & Izm2019 & 0.01 & 0.1 \\
\hline $15042+3641$ & HDS2124 & 2011.390 & 1 & 0.256 & 0.010 & 70.2 & 2.7 & & & & & \\
\hline $15049+3428$ & STF1908 & 2011.461 & 1 & 1.338 & 0.007 & $332.5^{*}$ & 0.3 & & & & & \\
\hline $15077+4632$ & COU1761 & 2011.392 & 1 & 1.156 & 0.007 & $265.9^{*}$ & 0.3 & & & & & \\
\hline $15078+3956$ & COU1271 & 2011.384 & 1 & 0.393 & 0.007 & $191.2^{*}$ & 0.5 & & & Cou1999b & 0.01 & -22.3 \\
\hline $15078+3956$ & COU1271 & 2012.359 & 1 & 0.383 & 0.009 & 190.2 & 0.5 & & & Cou1999b & 0.00 & -26.0 \\
\hline $15088+4014$ & COU1272AB & 2012.359 & 1 & 0.286 & 0.013 & 54.7 & 0.8 & & & & & \\
\hline $15088+4014$ & COU1272A,BC & 2012.359 & 1 & 1.275 & 0.028 & 177.0 & 0.9 & & & & & \\
\hline $15092+2809$ & A690 & 2011.384 & 1 & - & - & - & - & & NR & & & \\
\hline $15092+3508$ & COU972 & 2011.395 & 1 & 1.438 & 0.007 & $323.8^{*}$ & 0.3 & & & & & \\
\hline $15105+4800$ & HDS2131AB & 2011.461 & 1 & 0.240 & 0.023 & 152.9 & 3.3 & & & & & \\
\hline $15105+4800$ & HDS2131AC & 2011.461 & 1 & 3.508 & 0.024 & 59.8 & 0.4 & & & & & \\
\hline $15106+2021$ & HU144 & 2012.359 & 1 & 0.143 & 0.040 & $140.6^{*}$ & 8.0 & & & & & \\
\hline $15109+1749$ & COU64 & 2011.458 & 1 & 1.094 & 0.007 & 22.6 & 0.3 & & & & & \\
\hline $15121+1859$ & COU189 & 2011.469 & 1 & 0.466 & 0.011 & $140.5^{*}$ & 0.3 & & & & & \\
\hline $15127+4835$ & POP1220 & 2011.461 & 1 & 0.731 & 0.016 & $307.3^{*}$ & 0.3 & & & & & \\
\hline $15135+3244$ & ES2476 & 2012.474 & 1 & 1.307 & 0.014 & 25.4 & 3.2 & & & & & \\
\hline $15135+3244$ & ES2476 & 2012.474 & 2 & 1.325 & 0.040 & 27.2 & 1.3 & & & & & \\
\hline $15136+3453$ & HO60 & 2011.384 & 1 & 0.204 & 0.015 & $76.3^{*}$ & 1.6 & & & Doc2017f & 0.01 & 9.1 \\
\hline $15143+1959$ & COU102 & 2012.487 & 1 & 1.142 & 0.008 & $93.9^{*}$ & 0.3 & & & & & \\
\hline $15143+1959$ & COU102 & 2012.359 & 1 & 1.181 & 0.007 & $95.1^{*}$ & 0.3 & & & & & \\
\hline $15149+3818$ & STF1926 & 2011.392 & 1 & 0.365 & 0.044 & $226.7^{*}$ & ${ }^{*} 1.0$ & & & & & \\
\hline $15168+4100$ & ES1551 & 2011.461 & 1 & 1.313 & 0.007 & $332.4^{*}$ & 0.9 & & & & & \\
\hline $15175+3241$ & COU793 & 2011.392 & 1 & 1.452 & 0.008 & $60.8^{*}$ & 0.3 & & & & & \\
\hline $15175+4804$ & COU1762 & 2011.390 & 1 & 1.122 & 0.007 & 70.9 & 0.5 & & & & & \\
\hline $15181+3245$ & COU794 & 2011.384 & 1 & 0.194 & 0.040 & 135.4 & 8.0 & & & & & \\
\hline $15181+3507$ & HO61 & 2012.474 & 2 & 2.449 & 0.015 & $255.8^{*}$ & 0.7 & & & & & \\
\hline $15183+2650$ & STF1932 & 2011.469 & 1 & 1.621 & 0.008 & $263.9^{*}$ & 0.3 & & & Izm2019 & 0.01 & 9.1 \\
\hline $15186+2356$ & COU307 & 2011.458 & 1 & 0.317 & 0.007 & 3.8 & 0.7 & & & Lin2017c & -0.04 & 2.0 \\
\hline $15186+2356$ & COU307 & 2012.359 & 1 & 0.332 & 0.007 & 1.2 & 1.4 & & & Lin2017c & -0.02 & 0.3 \\
\hline $15188+3627$ & HDS2152 & 2011.384 & 1 & 1.143 & 0.007 & $223.9^{*}$ & 0.3 & & & & & \\
\hline $15195+2515$ & COU308 & 2011.392 & 1 & 1.413 & 0.007 & $355.3^{*}$ & 0.3 & & & & & \\
\hline $15200+4603$ & ES75AB,C & 2011.392 & 1 & 4.241 & 0.021 & $32.5^{*}$ & 0.3 & & & & & \\
\hline $15200+4603$ & ES75AB,C & 2011.392 & 2 & 4.242 & 0.021 & $32.4^{*}$ & 0.3 & & & & & \\
\hline $15200+4603$ & A1631 & 2011.392 & 1 & 0.767 & 0.007 & $253.8^{*}$ & 0.3 & & & & & \\
\hline $15206+1523$ & HU1160 & 2011.461 & 1 & 0.249 & 0.015 & 144.8 & 2.0 & & & Hrt2009 & -0.04 & 13.2 \\
\hline $15206+4656$ & HDS2155 & 2011.469 & 1 & 1.010 & 0.007 & $194.6^{*}$ & 0.3 & & & & & \\
\hline
\end{tabular}




\begin{tabular}{|c|c|c|c|c|c|c|c|c|c|c|c|c|}
\hline WDS & Name & Epoch & Bin. & $\begin{array}{c}\rho \\
(")\end{array}$ & $\begin{array}{l}\sigma_{\rho} \\
(")\end{array}$ & $\begin{array}{c}\theta \\
\left(^{\circ}\right)\end{array}$ & $\begin{array}{l}\sigma_{\theta} \\
\left(^{\circ}\right)\end{array}$ & $\Delta m$ & Notes & Orbit & $\begin{array}{r}\Delta \rho(\mathrm{O}-\mathrm{C}) \\
(")\end{array}$ & $\begin{array}{r}\Delta \theta(\mathrm{O}-\mathrm{C}) \\
\left({ }^{\circ}\right)\end{array}$ \\
\hline $15208+3459$ & HO62 & 2011.469 & 1 & 1.452 & 0.007 & $103.1^{*}$ & ${ }^{*} 0.3$ & & & & & \\
\hline $15209+4540$ & HDS2157 & 2011.461 & 1 & 0.695 & 0.011 & $279.4^{*}$ & ${ }^{*} 0.3$ & & & & & \\
\hline $15211+1640$ & A2229 & 2011.469 & 1 & 1.559 & 0.008 & 44.7 & 0.3 & & & & & \\
\hline $15223+4203$ & COU1440 & 2011.461 & 1 & 0.917 & 0.010 & 175.2 & 1.2 & & & & & \\
\hline $15224+3210$ & COU795 & 2011.469 & 1 & 0.694 & 0.007 & $324.3^{*}$ & ${ }^{*} 0.8$ & & & & & \\
\hline $15232+3017$ & STF1937 & 2012.487 & 1 & 0.661 & 0.007 & $189.5^{*}$ & * 0.5 & & & Mut2010b & 0.00 & 2.2 \\
\hline $15232+3017$ & STF1937 & 2011.461 & 1 & 0.638 & 0.007 & $180.6^{*}$ & ${ }^{*} 0.4$ & & & Mut2010b & 0.00 & 1.2 \\
\hline $15233+3619$ & A1367 & 2011.384 & 1 & 0.670 & 0.007 & $333.3^{*}$ & * 0.3 & & & & & \\
\hline $15233+4022$ & COU1441 & 2012.359 & 1 & 0.208 & 0.010 & 23.9 & 0.9 & & & & & \\
\hline $15245+3723$ & STF1938Ba,Bb & 2012.474 & 2 & 2.289 & 0.015 & $5.4^{*}$ & 0.3 & & & Izm2019 & 0.01 & 0.6 \\
\hline $15247+2318$ & COU105 & 2012.474 & 2 & 2.069 & 0.056 & 89.0 & 0.7 & & & & & \\
\hline $15255+1546$ & HEI381 & 2011.469 & 1 & 0.277 & 0.015 & 181.2 & 1.8 & & & & & \\
\hline $15271+2355$ & A82 & 2011.461 & 1 & 0.827 & 0.007 & $172.6^{*}$ & * 0.6 & & & & & \\
\hline $15294+4041$ & COU1273 & 2011.384 & 1 & 1.137 & 0.007 & 141.3 & 0.3 & & & & & \\
\hline $15307+2805$ & COU796 & 2011.384 & 1 & 0.370 & 0.007 & 115.7 & 0.6 & & & & & \\
\hline $15318+4054$ & $\mathrm{~A} 1634 \mathrm{AB}$ & 2011.461 & 1 & - & - & - & - & & NR & & & \\
\hline $15319+1623$ & A2075 & 2011.461 & 1 & 0.471 & 0.007 & $95.2^{*}$ & 0.7 & & & & & \\
\hline $15329+3122$ & COU610 & 2011.461 & 1 & 0.839 & 0.012 & $199.6^{*}$ & * 0.3 & & & & & \\
\hline $15331+3113$ & COU611 & 2011.392 & 1 & 0.670 & 0.007 & $96.4^{*}$ & 0.3 & & & & & \\
\hline $15340+3320$ & COU797 & 2011.384 & 1 & 1.767 & 0.009 & $202.2^{*}$ & * 0.3 & & & & & \\
\hline $15344+3201$ & HU746 & 2012.474 & 2 & 1.984 & 0.021 & 36.9 & 0.3 & & & & & \\
\hline $15347+2655$ & COU798 & 2012.487 & 1 & 0.179 & 0.040 & $203.8^{*}$ & 8.0 & & & Doc2010h & -0.01 & -10.2 \\
\hline $15360+3948$ & STT298 & 2012.495 & 1 & 1.152 & 0.007 & $181.8^{*}$ & * 0.3 & & & Sod1999 & -0.01 & 0.6 \\
\hline $15362+4915$ & HDS2194 & 2011.469 & 1 & 0.115 & 0.040 & 148.5 & 8.0 & & & & & \\
\hline $15363+4300$ & COU1444 & 2011.469 & 1 & 0.961 & 0.007 & $310.3^{*}$ & ${ }^{*} 0.5$ & & & & & \\
\hline $15371+2646$ & HDS2199AB & 2012.635 & 1 & 0.396 & 0.007 & 170.7 & 0.5 & & & & & \\
\hline $15371+2646$ & HDS2199AC & 2011.469 & 1 & 2.093 & 0.012 & 62.9 & 0.3 & & $\mathrm{ND}$ & & & \\
\hline $15376+2110$ & HU578 & 2011.469 & 1 & 1.209 & 0.007 & $136.3^{*}$ & $* 0.3$ & & & & & \\
\hline $15376+3427$ & COU975 & 2011.384 & 1 & 0.948 & 0.007 & 341.1 & 0.3 & & & & & \\
\hline $15376+3427$ & COU975 & 2011.469 & 1 & 0.951 & 0.007 & $340.7^{*}$ & $* 0.3$ & & & & & \\
\hline $15382+3615$ & STF1964CD & 2012.487 & 1 & 1.574 & 0.008 & $20.6^{*}$ & 0.3 & & & Dru1995 & 0.06 & 1.2 \\
\hline $15382+3615$ & STF1964CD & 2012.495 & 1 & 1.569 & 0.008 & $20.4^{*}$ & 0.4 & & & Dru1995 & 0.05 & 1.0 \\
\hline $15389+3401$ & COU976 & 2011.384 & 1 & 1.228 & 0.007 & $128.2^{*}$ & * 0.4 & & & & & \\
\hline $15390+2545$ & COU612 & 2012.487 & 1 & 0.266 & 0.011 & $348.4^{*}$ & * 0.9 & & & Doc2011h & -0.02 & $-1.9^{Q}$ \\
\hline $15396+7959$ & STF1989 & 2011.518 & 1 & 0.666 & 0.007 & $22.7^{*}$ & 0.3 & & & Hrt2008 & 0.04 & -0.4 \\
\hline $15402+4041$ & COU1274 & 2011.384 & 1 & 0.949 & 0.007 & $95.5^{*}$ & 0.6 & & & & & \\
\hline $15406+3128$ & COU613 & 2012.487 & 1 & - & - & - & - & & NR & & & \\
\hline $15422+7256$ & MLR192 & 2011.518 & 1 & 1.031 & 0.007 & $65.9^{*}$ & 0.6 & & & & & \\
\hline $15444+3942$ & A1635 & 2011.469 & 1 & - & - & - & - & & $\mathrm{NR}$ & & & \\
\hline $15451+2936$ & COU614 & 2011.469 & 1 & 0.293 & 0.007 & $214.1^{*}$ & $* 0.4$ & & & & & \\
\hline $15473+7327$ & MLR193 & 2011.518 & 1 & 0.567 & 0.007 & 181.3 & 0.5 & & & & & \\
\hline $15473+7327$ & MLR193 & 2011.518 & 1 & 0.567 & 0.007 & 181.3 & 0.5 & & & & & \\
\hline $15474+1851$ & COU192 & 2011.469 & 1 & 1.897 & 0.009 & $187.1^{*}$ & * 0.3 & & & & & \\
\hline $15478+3301$ & HDS2225 & 2011.384 & 1 & 0.159 & 0.040 & 128.5 & 8.0 & & & & & \\
\hline $15479+4159$ & A1638 & 2011.458 & 1 & 0.261 & 0.007 & $229.4^{*}$ & $* 0.7$ & & & & & \\
\hline $15489+2314$ & HDS2227 & 2011.469 & 1 & 0.627 & 0.007 & $287.3^{*}$ & $* 0.7$ & & & & & \\
\hline $15500+6012$ & HDS2229 & 2011.518 & 1 & 0.224 & 0.016 & $184.6^{*}$ & $* 0.9$ & & & & & \\
\hline $15501+3046$ & HDS2230 & 2011.384 & 1 & 1.779 & 0.009 & $313.0^{*}$ & $* 0.3$ & & & & & \\
\hline $15503+3449$ & A1858 & 2011.458 & 1 & 0.349 & 0.007 & $282.8^{*}$ & * 0.5 & & & & & \\
\hline $15509+1911$ & A2078 & 2011.524 & 1 & 1.091 & 0.007 & $164.7^{*}$ & $* 0.6$ & & & & & \\
\hline $15521+0528$ & A1128 & 2011.524 & 1 & 1.545 & 0.008 & $350.1^{*}$ & $* 0.4$ & & & & & \\
\hline $15521+1052$ & BAG7 & 2011.524 & 1 & 0.176 & 0.040 & 353.0 & 8.0 & & & Doc2019c & 0.10 & -0.6 \\
\hline $15530-0218$ & RST5035 & 2011.524 & 1 & 0.480 & 0.007 & $115.1^{*}$ & * 1.5 & & & & & \\
\hline
\end{tabular}




\begin{tabular}{|c|c|c|c|c|c|c|c|c|c|c|c|c|}
\hline WDS & Name & Epoch & Bin. & $\begin{array}{c}\rho \\
(")\end{array}$ & $\begin{array}{l}\sigma_{\rho} \\
(")\end{array}$ & $\begin{array}{c}\theta \\
\left({ }^{\circ}\right)\end{array}$ & $\begin{array}{l}\sigma_{\theta} \\
\left({ }^{\circ}\right)\end{array}$ & $\Delta m$ & Notes & Orbit & $\begin{array}{r}\Delta \rho(\mathrm{O}-\mathrm{C}) \\
(")\end{array}$ & $\begin{array}{r}\Delta \theta(\mathrm{O}-\mathrm{C}) \\
\left({ }^{\circ}\right)\end{array}$ \\
\hline $15530+6007$ & HU913AB & 2011.518 & 1 & 0.406 & 0.007 & $310.3^{*}$ & * 0.3 & & & & & \\
\hline $15542+1659$ & A2080 & 2011.521 & 1 & 0.346 & 0.015 & $101.5^{*}$ & $* 0.4$ & & & Hrt2008 & -0.03 & -3.1 \\
\hline $15543+0528$ & A1130 & 2011.524 & 1 & 1.967 & 0.010 & $329.2^{*}$ & * 0.3 & & & & & \\
\hline $15568+1229$ & STF1988 & 2011.526 & 1 & 1.849 & 0.010 & $250.5^{*}$ & * 0.9 & & & & & \\
\hline $15583+4019$ & COU1448 & 2011.384 & 1 & 1.096 & 0.009 & $257.0^{*}$ & * 0.3 & & & & & \\
\hline $15583+4019$ & COU1448 & 2011.458 & 1 & 1.106 & 0.007 & $76.4^{*}$ & 0.7 & & & & & \\
\hline $15595+2555$ & JEF2 & 2011.458 & 1 & - & - & - & - & & NR & & & \\
\hline $15595+2555$ & JEF2 & 2012.487 & 1 & - & - & - & - & & NR & & & \\
\hline $15595+2555$ & JEF2 & 2011.458 & 1 & 0.134 & 0.040 & 134.8 & 8.0 & & & & & \\
\hline $15596+4440$ & COU1764 & 2012.496 & 1 & 1.312 & 0.007 & $207.7^{*}$ & * 0.3 & & & & & \\
\hline $16001+3625$ & COU1138 & 2011.458 & 1 & 0.187 & 0.040 & 170.1 & 8.0 & & & & & \\
\hline $16001+3625$ & COU1138 & 2012.496 & 1 & 0.243 & 0.037 & 181.6 & 7.1 & & & & & \\
\hline $16003+1140$ & A1639AB & 2011.526 & 1 & 0.264 & 0.007 & $93.0^{*}$ & 1.3 & & & Zir2012b & 0.03 & 4.9 \\
\hline $16003+5856$ & STF2006 & 2011.515 & 1 & 1.483 & 0.007 & $178.2^{*}$ & * 0.3 & & & & & \\
\hline $16004+5431$ & HU154 & 2012.528 & 1 & 1.537 & 0.008 & 117.1 & 0.3 & & & & & \\
\hline $16004+5431$ & HU154 & 2012.528 & 1 & 1.555 & 0.013 & 117.4 & 0.4 & & & & & \\
\hline $16004+7331$ & MLR195 & 2011.515 & 1 & 1.237 & 0.007 & $299.4^{*}$ & * 0.3 & & & & & \\
\hline $16007+0051$ & RST5403 & 2011.521 & 1 & 0.861 & 0.007 & $55.5^{*}$ & 0.5 & & & & & \\
\hline $16009+1918$ & A2081 & 2012.487 & 1 & 2.392 & 0.012 & $324.4^{*}$ & * 0.3 & & & & & \\
\hline $16011+6531$ & HU1170 & 2012.523 & 1 & 0.995 & 0.007 & $148.9^{*}$ & $* 0.3$ & & & & & \\
\hline $16012+0136$ & HEI792 & 2011.521 & 1 & 1.856 & 0.009 & 78.7 & 0.3 & & & & & \\
\hline $16013-0658$ & BU623 & 2011.521 & 1 & 0.765 & 0.007 & $223.9^{*}$ & * 0.3 & & & & & \\
\hline $16022+6004$ & MLR45 & 2011.515 & 1 & 0.398 & 0.007 & $73.3^{*}$ & 0.3 & & & & & \\
\hline $16026+2637$ & HDS2262 & 2011.469 & 1 & 0.325 & 0.014 & $266.5^{*}$ & * 0.8 & & & & & \\
\hline $16032+2851$ & STF2004 & 2012.479 & 1 & 1.835 & 0.009 & $278.2^{*}$ & $* 0.3$ & & & & & \\
\hline $16038+5906$ & MLR535 & 2011.518 & 1 & 1.599 & 0.010 & $130.6^{*}$ & * 0.3 & & & & & \\
\hline $16040+2515$ & TRN1 & 2012.479 & 1 & 0.207 & 0.011 & 4.8 & 1.2 & & & & & \\
\hline $16043+3230$ & HDS2267 & 2012.496 & 1 & 0.438 & 0.007 & $329.6^{*}$ & * 0.6 & & & & & \\
\hline $16044+3625$ & HDS2268 & 2011.469 & 1 & 0.347 & 0.007 & $190.5^{*}$ & * 1.9 & & & & & \\
\hline $16048+2028$ & COU194 & 2011.458 & 1 & 1.902 & 0.010 & $339.9^{*}$ & * 0.3 & & & & & \\
\hline $16048+2028$ & COU194 & 2012.496 & 1 & 1.877 & 0.009 & $160.1^{*}$ & * 0.3 & & & & & \\
\hline $16048+7016$ & HDS2269 & 2012.528 & 1 & 0.218 & 0.108 & 253.1 & 9.0 & & & & & \\
\hline $16048+7016$ & HDS2269 & 2012.528 & 1 & 0.286 & 0.007 & $249.9^{*}$ & * 1.4 & & & & & \\
\hline $16053+5810$ & KUI71 & 2011.515 & 1 & 0.638 & 0.007 & $11.2^{*}$ & 0.5 & & & & & \\
\hline $16053+5810$ & KUI71 & 2012.523 & 1 & 0.610 & 0.007 & 11.5 & 0.7 & 0.05 & & & & \\
\hline $16053+5810$ & KUI71 & 2012.523 & 1 & 0.614 & 0.039 & 13.0 & 2.3 & & & & & \\
\hline $16053+5810$ & KUI71 & 2012.523 & 1 & 0.621 & 0.007 & $11.8^{*}$ & 0.3 & & & & & \\
\hline $16057+0255$ & A 2178 & 2011.521 & 1 & 1.246 & 0.007 & $327.7^{*}$ & * 0.3 & & & & & \\
\hline $16062+1809$ & COU2389 & 2011.469 & 1 & 1.101 & 0.007 & $56.2^{*}$ & 0.3 & & & & & \\
\hline $16065+5604$ & YSC110 & 2012.523 & 1 & - & - & - & - & & NR & & & \\
\hline $16065+7547$ & HU916 & 2011.518 & 1 & 0.300 & 0.012 & $169.6^{*}$ & * 0.8 & & & & & \\
\hline $16079+1425$ & A1798 & 2011.510 & 1 & 0.199 & 0.040 & $350.8^{*}$ & * 8.0 & & & USN2002 & 0.04 & 8.7 \\
\hline $16080+4523$ & BU355 & 2012.496 & 1 & 0.146 & 0.040 & $280.1^{*}$ & * 8.0 & & & & & \\
\hline $16092-0549$ & RST4559 & 2011.526 & 1 & 0.931 & 0.007 & $295.7^{*}$ & * 0.3 & & & & & \\
\hline $16094+1305$ & HEI385 & 2011.510 & 1 & 1.931 & 0.010 & $189.7^{*}$ & * 0.3 & & & & & \\
\hline $16096+2658$ & HDS2282 & 2012.517 & 1 & 0.405 & 0.007 & $55.1^{*}$ & 0.6 & & & & & \\
\hline $16097-0633$ & RST3932 & 2011.526 & 1 & 0.238 & 0.010 & $154.7^{*}$ & * 1.2 & & & & & \\
\hline $16097+4900$ & COU1919 & 2012.517 & 1 & 0.737 & 0.007 & $134.0^{*}$ & * 0.3 & & & & & \\
\hline $16107+4019$ & COU1277 & 2011.458 & 1 & 0.691 & 0.008 & $171.2^{*}$ & * 0.6 & & & & & \\
\hline $16109+6554$ & MLR179 & 2011.515 & 1 & 0.284 & 0.007 & $333.4^{*}$ & * 2.4 & & & & & \\
\hline $16118-0041$ & RST5046 & 2011.526 & 1 & 1.044 & 0.008 & $358.2^{*}$ & * 0.3 & & & & & \\
\hline $16118+3424$ & STT306 & 2012.479 & 1 & 0.222 & 0.007 & $5.4^{*}$ & 1.1 & & & & & \\
\hline $16121+3303$ & HU1171 & 2012.517 & 1 & 1.129 & 0.007 & $305.1^{*}$ & * 0.6 & & & & & \\
\hline $16126+5146$ & YSC112 & 2012.496 & 1 & 0.761 & 0.007 & 154.1 & 0.5 & & & & & \\
\hline
\end{tabular}




\begin{tabular}{|c|c|c|c|c|c|c|c|c|c|c|c|c|}
\hline WDS & Name & Epoch & Bin. & $\begin{array}{c}\rho \\
(")\end{array}$ & $\begin{array}{l}\sigma_{\rho} \\
(")\end{array}$ & $\begin{array}{c}\theta \\
\left({ }^{\circ}\right)\end{array}$ & $\begin{array}{l}\sigma_{\theta} \\
\left({ }^{\circ}\right)\end{array}$ & $\Delta m$ & Notes & Orbit & $\begin{array}{r}\Delta \rho(\mathrm{O}-\mathrm{C}) \\
(")\end{array}$ & $\begin{array}{r}\Delta \theta(\mathrm{O}-\mathrm{C}) \\
\left({ }^{\circ}\right)\end{array}$ \\
\hline $16126+5146$ & YSC112 & 2012.496 & 1 & 0.756 & 0.007 & 154.8 & 0.3 & 3.30 & & & & \\
\hline $16132+7339$ & MLR196 & 2011.515 & 1 & 0.730 & 0.007 & $235.9^{*}$ & * 0.3 & & & & & \\
\hline $16132+7339$ & MLR196 & 2012.531 & 1 & 0.722 & 0.007 & 55.7 & 1.2 & & & & & \\
\hline $16132+7339$ & MLR196 & 2012.531 & 1 & 0.724 & 0.007 & $56.5^{*}$ & 0.9 & & & & & \\
\hline $16135+3202$ & HDS2293 & 2011.469 & 1 & 0.402 & 0.015 & $116.2^{*}$ & * 2.5 & & & & & \\
\hline $16135+3202$ & HDS2293 & 2011.469 & 1 & - & - & - & - & & NR & & & \\
\hline $16135+7147$ & A1136 & 2011.518 & 1 & 0.751 & 0.010 & $8.9^{*}$ & 0.3 & & & & & \\
\hline $16143+2042$ & YSC58 & 2012.479 & 1 & - & - & - & - & & NR & & & \\
\hline $16152-0709$ & RST3938 & 2011.521 & 1 & 1.227 & 0.010 & 28.2 & 0.4 & & & & & \\
\hline $16152+3443$ & HDS2298 & 2011.458 & 1 & 0.355 & 0.024 & $190.6^{*}$ & * 1.7 & & & & & \\
\hline $16160+6228$ & MLR53 & 2012.523 & 1 & 1.863 & 0.009 & 148.5 & 1.2 & & & & & \\
\hline $16160+6228$ & MLR53 & 2012.523 & 1 & 1.840 & 0.010 & $147.4^{*}$ & ${ }^{k} 0.3$ & & & & & \\
\hline $16162+6126$ & MLR49 & 2012.528 & 1 & 0.354 & 0.007 & $90.0^{*}$ & 0.3 & & & & & \\
\hline $16164+4327$ & COU1450 & 2011.469 & 1 & 1.331 & 0.007 & $61.9^{*}$ & 0.3 & & & & & \\
\hline $16166+4001$ & COU1278 & 2012.496 & 1 & 1.252 & 0.007 & $155.7^{*}$ & ${ }^{*} 0.3$ & & & & & \\
\hline $16168+0051$ & A2182 & 2011.526 & 1 & 1.461 & 0.007 & $90.0^{*}$ & 0.3 & & & & & \\
\hline $16172+3341$ & COU980 & 2011.469 & 1 & 0.543 & 0.007 & $341.4^{*}$ & ${ }^{*} 1.2$ & & & & & \\
\hline $16178+0417$ & HEI891 & 2011.521 & 1 & 1.252 & 0.007 & $294.6^{*}$ & ${ }^{*} 0.3$ & & & & & \\
\hline $16178+3148$ & HDS2303AB & 2012.517 & 1 & 0.308 & 0.007 & 122.7 & 0.3 & 3.64 & & & & \\
\hline $16178+3148$ & HDS2303AC & 2012.517 & 1 & 1.573 & 0.008 & 37.9 & 0.3 & & & & & \\
\hline $16192+2553$ & COU412 & 2012.487 & 1 & 0.954 & 0.007 & $82.7^{*}$ & 0.3 & & & & & \\
\hline $16199+2238$ & LAW4Aa,Ab & 2012.487 & 1 & 0.277 & 0.007 & 28.3 & 0.7 & & & & & \\
\hline $16199+2238$ & LAW4Aa,Ab & 2012.479 & 1 & - & - & - & - & & NR & & & \\
\hline $16199+3600$ & COU981 & 2011.469 & 1 & 1.077 & 0.009 & $131.6^{*}$ & ${ }^{k} 0.3$ & & & & & \\
\hline $16211+0250$ & HEI794AB & 2011.521 & 2 & 0.855 & 0.018 & $58.0^{*}$ & 0.3 & & & & & \\
\hline $16211+0250$ & HEI794AC & 2011.521 & 2 & 3.749 & 0.037 & $33.8^{*}$ & 0.3 & & $\mathrm{ND}$ & & & \\
\hline $16212+2259$ & HU481 & 2012.517 & 1 & 0.362 & 0.007 & $257.8^{*}$ & ${ }^{k} 0.4$ & & & Hrt2010a & 0.03 & -2.5 \\
\hline $16212+2259$ & HU481 & 2012.517 & 1 & 0.357 & 0.007 & 259.3 & 0.3 & 2.45 & & Hrt2010a & 0.02 & -0.9 \\
\hline $16213+7434$ & HDS2310AB & 2011.515 & 1 & 0.410 & 0.014 & 102.8 & 1.7 & & & & & \\
\hline $16213+7434$ & HDS2310AB & 2012.523 & 1 & - & - & - & - & & NR & & & \\
\hline $16213+7434$ & HDS2310AC & 2011.515 & 1 & 2.698 & 0.013 & 26.7 & 0.3 & & $\mathrm{ND}$ & & & \\
\hline $16213+7434$ & HDS2310AC & 2012.523 & 1 & 2.706 & 0.014 & $25.4^{*}$ & 0.3 & & $\mathrm{ND}$ & & & \\
\hline $16213+7434$ & HDS2310AC & 2012.523 & 1 & 2.733 & 0.014 & 26.3 & 0.5 & & $\mathrm{ND}$ & & & \\
\hline $16216+3631$ & COU982 & 2012.517 & 1 & 0.280 & 0.007 & $237.1^{*}$ & ${ }^{*} 0.3$ & & & Hrt2009 & -0.02 & -2.2 \\
\hline $16220+3908$ & COU1279 & 2012.496 & 1 & 1.672 & 0.008 & $27.9^{*}$ & 0.3 & & & & & \\
\hline $16229+3803$ & COU1281 & 2012.517 & 1 & 0.215 & 0.007 & $202.8^{*}$ & ${ }^{k} 2.1$ & & & Doc $2012 \mathrm{~h}$ & 0.06 & -4.2 \\
\hline $16233+3251$ & COU619 & 2012.479 & 1 & 0.481 & 0.007 & $8.5^{*}$ & 0.4 & & & & & \\
\hline $16234-0859$ & HDS2316 & 2011.521 & 1 & - & - & - & - & & NR & & & \\
\hline $16235+3321$ & BU951AB,C & 2012.520 & 1 & 1.049 & 0.007 & 30.4 & 0.3 & & & & & \\
\hline $16235+3321$ & BU951AB,C & 2012.520 & 1 & 1.044 & 0.007 & $30.4^{*}$ & 0.4 & & & & & \\
\hline $16235+3321$ & VBS26AB & 2012.520 & 1 & 0.183 & 0.040 & $358.2^{*}$ & 8.0 & & & USN2002 & 0.06 & 0.4 \\
\hline $16238+6142$ & STF2054 & 2012.528 & 1 & 0.971 & 0.007 & $351.0^{*}$ & 0.3 & & & & & \\
\hline $16248+4441$ & YSC59AB & 2012.496 & 1 & - & - & - & - & & NR & & & \\
\hline $16252+4652$ & YSC60 & 2012.496 & 1 & - & - & - & - & & NR & & & \\
\hline $16253-0952$ & BU950 & 2011.521 & 1 & 1.042 & 0.007 & $343.7^{*}$ & 0.3 & & & & & \\
\hline $16257+4921$ & COU1920 & 2012.496 & 1 & 0.751 & 0.011 & $61.4^{*}$ & 0.5 & & & & & \\
\hline $16265+5037$ & COU2112 & 2012.520 & 1 & 0.565 & 0.007 & $75.7^{*}$ & 0.6 & & & & & \\
\hline $16270+3708$ & COU1139 & 2012.517 & 1 & 1.613 & 0.008 & $296.4^{*}$ & 0.3 & & & & & \\
\hline $16272+3521$ & COU984 & 2011.469 & 1 & 0.632 & 0.007 & $354.5^{*}$ & 0.5 & & & & & \\
\hline $16282+7614$ & MLR350 & 2011.518 & 1 & 0.906 & 0.007 & $228.5^{*}$ & 0.4 & & & & & \\
\hline $16287+3714$ & COU1140 & 2011.469 & 1 & 0.803 & 0.007 & $351.8^{*}$ & 0.3 & & & & & \\
\hline $16301+3353$ & HU1173 & 2012.517 & 1 & 0.207 & 0.009 & 60.8 & 2.3 & & & Hei1997 & -0.05 & -3.4 \\
\hline
\end{tabular}




\begin{tabular}{|c|c|c|c|c|c|c|c|c|c|c|c|c|}
\hline WDS & Name & Epoch & Bin. & $\begin{array}{c}\rho \\
(")\end{array}$ & $\begin{array}{l}\sigma_{\rho} \\
(")\end{array}$ & $\begin{array}{c}\theta \\
\left({ }^{\circ}\right)\end{array}$ & $\begin{array}{l}\sigma_{\theta} \\
\left(^{\circ}\right)\end{array}$ & $\Delta m$ & Notes & Orbit & $\begin{array}{r}\Delta \rho(\mathrm{O}-\mathrm{C}) \\
(")\end{array}$ & $\begin{array}{r}\Delta \theta(\mathrm{O}-\mathrm{C}) \\
\left({ }^{\circ}\right)\end{array}$ \\
\hline $16304+4044$ & HDS2331 & 2011.469 & 1 & 0.564 & 0.021 & 276.4 & 0.6 & & & & & \\
\hline $16304+4044$ & HDS2331 & 2011.469 & 1 & 0.553 & 0.010 & $276.7^{*}$ & ${ }^{k} 0.5$ & & & & & \\
\hline $16308-0218$ & RST5059 & 2011.521 & 1 & 1.339 & 0.007 & $172.1^{*}$ & ${ }^{k} 0.3$ & & & & & \\
\hline $16309+6318$ & TDS9943 & 2012.531 & 1 & 0.660 & 0.007 & $195.3^{*}$ & ${ }^{k} 0.4$ & & & & & \\
\hline $16309+6318$ & TDS9943 & 2012.531 & 1 & 0.677 & 0.018 & 193.5 & 1.2 & & & & & \\
\hline $16310+3514 \mathrm{AB}$ & HDS2332AB & 2012.547 & 1 & - & - & - & - & & NR & & & \\
\hline $16311+5756$ & A1138 & 2011.515 & 1 & 0.547 & 0.007 & 174.5 & 0.3 & & & & & \\
\hline $16312+5725$ & WOR32 & 2012.523 & 1 & 1.002 & 0.007 & $1.0^{*}$ & 0.3 & & & & & \\
\hline $16313+5557$ & MLR636 & 2011.515 & 1 & 0.577 & 0.007 & $270.1^{*}$ & ${ }^{*} 0.3$ & & & & & \\
\hline $16315+7237$ & HDS2334 & 2012.528 & 1 & 1.057 & 0.007 & 174.2 & 0.6 & & & & & \\
\hline $16315+7237$ & HDS2334 & 2012.528 & 1 & 1.081 & 0.011 & $175.1^{*}$ & 0.3 & & & & & \\
\hline $16315+7237$ & HDS2334 & 2011.518 & 1 & 1.020 & 0.007 & $175.3^{*}$ & ${ }^{k} 0.4$ & & & & & \\
\hline $16319+5259$ & A1862 & 2012.528 & 1 & 0.336 & 0.038 & 67.6 & 4.4 & & & & & \\
\hline $16319+5259$ & A1862 & 2012.528 & 1 & 0.401 & 0.007 & $63.0^{*}$ & 1.0 & & & & & \\
\hline $16322+6940$ & MLR180 & 2011.515 & 1 & 0.551 & 0.007 & $246.9^{*}$ & ${ }^{k} 0.4$ & & & & & \\
\hline $16326+2314$ & BU817 & 2012.547 & 1 & 0.942 & 0.007 & $328.1^{*}$ & ${ }^{k} 0.3$ & & & & & \\
\hline $16326+4007$ & STT313 & 2012.520 & 1 & 0.940 & 0.007 & $130.9^{*}$ & ${ }^{k} 0.5$ & & & & & \\
\hline $16330+0233$ & A 2234 & 2011.521 & 1 & 0.941 & 0.009 & $121.9^{*}$ & ${ }^{k} 0.9$ & & & & & \\
\hline $16330+0233$ & $\mathrm{~A} 2234$ & 2011.521 & 1 & 0.982 & 0.007 & 121.8 & 0.3 & 3.47 & & & & \\
\hline $16332-0956$ & RST3952 & 2011.526 & 1 & 2.069 & 0.010 & $92.9^{*}$ & 0.3 & & & & & \\
\hline $16335+3030$ & BU818 & 2011.521 & 1 & 1.576 & 0.028 & $238.8^{*}$ & ${ }^{*} 0.6$ & & & & & \\
\hline $16335+3030$ & BU818 & 2011.521 & 1 & 1.476 & 0.027 & 238.8 & 1.3 & 3.13 & & & & \\
\hline $16337+4520$ & COU1590 & 2012.520 & 1 & 1.209 & 0.007 & $29.4^{*}$ & 0.3 & & & & & \\
\hline $16339+7159$ & HDS2342 & 2011.518 & 1 & 0.324 & 0.007 & $264.9^{*}$ & ${ }^{k} 0.4$ & & & & & \\
\hline $16348+2145$ & WSI105 & 2012.517 & 1 & - & - & - & - & & NR & & & \\
\hline $16357+6716$ & MLR181 & 2011.518 & 1 & 1.379 & 0.007 & 105.0 & 0.3 & & & & & \\
\hline $16360+6237$ & MLR54 & 2011.518 & 1 & 1.522 & 0.008 & $57.6^{*}$ & 0.3 & & & & & \\
\hline $16366+5502$ & TDS824 & 2012.528 & 1 & 1.559 & 0.013 & $296.5^{*}$ & ${ }^{*} 0.3$ & & & & & \\
\hline $16366+6948$ & BAZ7 & 2011.515 & 1 & 0.490 & 0.022 & 87.2 & 4.5 & & & & & \\
\hline $16366+6948$ & BAZ7 & 2012.523 & 1 & 0.462 & 0.029 & 90.3 & 4.8 & & & & & \\
\hline $16367+4230$ & COU1451 & 2011.469 & 1 & 1.113 & 0.007 & $2.0^{*}$ & 0.4 & & & & & \\
\hline $16369+4547$ & COU1591 & 2012.520 & 1 & 0.553 & 0.007 & $19.2^{*}$ & 0.3 & & & & & \\
\hline $16371+1732$ & COU311 & 2012.479 & 1 & 0.334 & 0.007 & $101.9^{*}$ & ${ }^{*} 1.4$ & & & Cou1999b & -0.04 & -10.7 \\
\hline $16371+1732$ & COU311 & 2011.469 & 1 & 0.360 & 0.024 & 108.3 & 1.5 & & & Cou1999b & -0.02 & -3.6 \\
\hline $16376+4510$ & A1643 & 2012.547 & 1 & 0.780 & 0.007 & $151.5^{*}$ & ${ }^{*} 0.3$ & & & & & \\
\hline $16380+4831$ & COU1768 & 2012.496 & 1 & 0.613 & 0.007 & $300.5^{*}$ & ${ }^{k} 0.6$ & & & & & \\
\hline $16380+6910$ & HDS2353 & 2011.518 & 1 & 0.311 & 0.008 & $202.1^{*}$ & ${ }^{k} 0.5$ & & & & & \\
\hline $16381+3935$ & HDS2355 & 2012.547 & 1 & 0.590 & 0.007 & $326.5^{*}$ & ${ }^{*} 0.7$ & & & & & \\
\hline $16384+3514$ & COU985 & 2012.520 & 1 & 0.323 & 0.010 & $255.0^{*}$ & ${ }^{*} 0.6$ & & & Doc2019h & -0.00 & -0.7 \\
\hline $16386-0204$ & RST5062 & 2011.521 & 1 & 0.445 & 0.007 & $266.9^{*}$ & ${ }^{k} 1.1$ & & & & & \\
\hline $16386-0204$ & RST5062 & 2011.521 & 1 & 0.400 & 0.007 & 264.5 & 0.3 & 0.99 & & & & \\
\hline $16386+3820$ & STF2080 & 2012.496 & 1 & 0.782 & 0.007 & $240.5^{*}$ & ${ }^{k} 0.3$ & & & & & \\
\hline $16392+6819$ & YSC113 & 2012.523 & 1 & - & - & - & - & & NR & & & \\
\hline $16392+6819$ & YSC113 & 2012.528 & 1 & 0.866 & 0.015 & 333.3 & 0.5 & & & & & \\
\hline $16396+2344$ & COU414 & 2012.496 & 1 & 1.753 & 0.009 & $197.1^{*}$ & ${ }^{k} 0.3$ & & & & & \\
\hline $16397-0337$ & A26 & 2011.521 & 1 & 1.314 & 0.007 & 340.9 & 0.3 & 2.57 & & & & \\
\hline $16397-0337$ & A26 & 2011.521 & 1 & 1.331 & 0.007 & $340.9^{*}$ & ${ }^{k} 0.4$ & & & & & \\
\hline $16407+7323$ & MLR197AB & 2011.515 & 1 & 0.454 & 0.008 & $74.0^{*}$ & 0.8 & & & & & \\
\hline $16407+7323$ & MLR197AC & 2012.235 & 1 & 1.927 & 0.010 & $247.2^{*}$ & ${ }^{*} 0.3$ & & & & & \\
\hline $16412-0100$ & HDS2367 & 2011.524 & 1 & 0.799 & 0.007 & $68.1^{*}$ & 0.3 & & & & & \\
\hline
\end{tabular}




\begin{tabular}{|c|c|c|c|c|c|c|c|c|c|c|c|c|}
\hline WDS & Name & Epoch & Bin. & $\begin{array}{c}\rho \\
(")\end{array}$ & $\begin{array}{l}\sigma_{\rho} \\
(")\end{array}$ & $\begin{array}{c}\theta \\
\left({ }^{\circ}\right)\end{array}$ & $\begin{array}{l}\sigma_{\theta} \\
\left({ }^{\circ}\right)\end{array}$ & $\Delta m$ & Notes & Orbit & $\begin{array}{r}\Delta \rho(\mathrm{O}-\mathrm{C}) \\
(")\end{array}$ & $\begin{array}{r}\Delta \theta(\mathrm{O}-\mathrm{C}) \\
\left({ }^{\circ}\right)\end{array}$ \\
\hline $16413+1816$ & COU2512 & 2012.479 & 1 & 0.372 & 0.007 & $181.8^{*}$ & 0.5 & & & & & \\
\hline $16413+3006$ & A349 & 2012.517 & 1 & 0.672 & 0.007 & $133.4^{*}$ & 0.3 & & & Hrt2014b & 0.01 & -0.6 \\
\hline $16420+5711$ & HDS2371 & 2011.518 & 1 & 0.861 & 0.007 & $71.8^{*}$ & 0.5 & & & & & \\
\hline $16420+7353$ & MLR198 & 2011.515 & 1 & 0.210 & 0.010 & $53.8^{*}$ & 1.6 & & & Hrt2014b & -0.02 & -7.4 \\
\hline $16420+7353$ & MLR198 & 2012.523 & 1 & 0.196 & 0.040 & 50.8 & 8.0 & & & Hrt2014b & -0.04 & $-7.3^{Q}$ \\
\hline $16421-0847$ & RST3956 & 2011.524 & 1 & 2.220 & 0.207 & $201.7^{*}$ & 10.7 & & & & & \\
\hline $16422+3753$ & HDS2372 & 2012.547 & 1 & 0.196 & 0.040 & $241.8^{*}$ & 8.0 & & & & & \\
\hline $16422+4112$ & STF2091 & 2012.547 & 1 & 0.379 & 0.007 & $328.2^{*}$ & 0.7 & & & Zir2014a & 0.00 & 0.2 \\
\hline $16428+7122$ & HDS2373 & 2011.518 & 1 & 0.166 & 0.040 & 180.6 & 8.0 & & & & & \\
\hline $16429+4814$ & COU1769 & 2012.496 & 1 & 0.553 & 0.007 & $181.6^{*}$ & 0.3 & & & & & \\
\hline $16430+5533$ & TDT45 & 2012.528 & 1 & - & - & - & - & & NR & & & \\
\hline $16433-0953$ & RST3958 & 2011.524 & 1 & 0.900 & 0.022 & $145.3^{*}$ & 0.7 & & & & & \\
\hline $16434+3539$ & COU986 & 2012.479 & 1 & 1.272 & 0.014 & $221.3^{*}$ & 0.4 & & & & & \\
\hline $16434+6120$ & TDS831 & 2012.528 & 1 & 1.084 & 0.018 & $215.8^{*}$ & 1.0 & & & & & \\
\hline $16435-0807$ & WRH26 & 2011.526 & 1 & 0.331 & 0.013 & 168.3 & 1.7 & & & & & \\
\hline $16437+5544$ & MLR570 & 2012.523 & 1 & 1.264 & 0.007 & $350.0^{*}$ & 0.3 & & & & & \\
\hline $16439+4329$ & D15 & 2011.469 & 1 & 0.413 & 0.007 & $2.5^{*}$ & 0.9 & & & Alz2007 & 0.03 & -0.8 \\
\hline $16442+2331$ & STF2094 & 2012.553 & 1 & 1.159 & 0.013 & 72.5 & 0.4 & & & & & \\
\hline $16442+2331$ & STF2094 & 2012.553 & 1 & 1.142 & 0.007 & 72.9 & 0.8 & 0.84 & & & & \\
\hline $16446+7145$ & MLR182Aa,Ab & 2011.515 & 1 & 0.310 & 0.012 & 92.1 & 0.7 & & & Hei1997 & 0.00 & 32.7 \\
\hline $16446+7145$ & MLR182Aa,Ab & 2012.528 & 1 & 0.311 & 0.009 & $92.7^{*}$ & 0.6 & & & Hei1997 & 0.01 & 35.5 \\
\hline $16446+7145$ & MLR182Aa,Ab & 2012.528 & 1 & 0.303 & 0.007 & 94.0 & 2.2 & & & Hei1997 & 0.00 & 36.8 \\
\hline $16447+3148$ & COU804 & 2011.469 & 1 & 0.212 & 0.007 & 30.2 & 2.2 & & & & & \\
\hline $16449+0039$ & RST5416 & 2011.524 & 1 & 0.769 & 0.018 & $101.9^{*}$ & 1.2 & & & & & \\
\hline $16450+2928$ & COU490 & 2012.479 & 1 & - & - & - & - & & NR & & & \\
\hline $16450+3842$ & COU1284 & 2012.517 & 1 & 0.162 & 0.040 & $166.8^{*}$ & 8.0 & & & Cou1999b & 0.05 & $29.3^{Q}$ \\
\hline $16453+2435$ & HDS2376 & 2012.520 & 1 & 0.641 & 0.009 & $257.6^{*}$ & 0.4 & & & & & \\
\hline $16458-0828$ & HDS2378 & 2011.526 & 1 & 0.318 & 0.007 & $148.1^{*}$ & 0.8 & & & & & \\
\hline $16471+7435$ & TDS835 & 2012.528 & 1 & 1.191 & 0.007 & $115.8^{*}$ & 0.7 & & & & & \\
\hline $16472+6454$ & MLR61 & 2011.518 & 1 & - & - & - & - & & NR & & & \\
\hline $16476+4255$ & GII3 & 2011.179 & 1 & 1.303 & 0.028 & 182.3 & 1.3 & & & & & \\
\hline $16483+0244$ & BU43 & 2011.524 & 1 & 1.411 & 0.008 & $54.6^{*}$ & 0.3 & & & & & \\
\hline $16486+2516$ & COU491 & 2012.479 & 1 & 0.769 & 0.007 & $42.0^{*}$ & 0.4 & & & & & \\
\hline $16488+6752$ & MLR183 & 2011.518 & 1 & 0.728 & 0.013 & 27.8 & 0.4 & & & & & \\
\hline $16492+4559$ & A1866BC & 2011.559 & 1 & 0.336 & 0.007 & $258.1^{*}$ & 0.3 & 0.16 & & DRs2012 & 0.05 & -3.7 \\
\hline $16492+4559$ & A1866BC & 2012.520 & 1 & 0.342 & 0.007 & 264.9 & 0.3 & 0.10 & & DRs2012 & 0.05 & -1.4 \\
\hline $16492+4559$ & A1866BC & 2012.520 & 1 & 0.335 & 0.007 & 264.3 & 0.3 & 0.23 & & DRs2012 & 0.05 & -2.0 \\
\hline $16492+4559$ & A1866BC & 2012.520 & 1 & 0.306 & 0.007 & 259.9 & 0.3 & 0.36 & & DRs2012 & 0.02 & -6.4 \\
\hline $16492+4559$ & BU627A,BC & 2011.559 & 1 & 2.133 & 0.011 & $40.7^{*}$ & 0.3 & & & & & \\
\hline $16492+4559$ & BU627A,BC & 2012.520 & 1 & 2.092 & 0.010 & $41.2^{*}$ & 0.3 & & & & & \\
\hline $16492+4559$ & BU627A,BC & 2012.520 & 1 & 2.106 & 0.011 & 41.4 & 0.3 & 3.95 & & & & \\
\hline $16497+5816$ & MLR430 & 2011.515 & 1 & 0.148 & 0.040 & 99.4 & 8.0 & & & Lin2019b & -0.02 & -15.8 \\
\hline $16509+4259$ & A 575 & 2011.455 & 1 & 0.715 & 0.007 & $149.8^{*}$ & 0.6 & & & & & \\
\hline $16509+4445$ & COU1592 & 2011.455 & 1 & 0.565 & 0.007 & 47.8 & 0.7 & & & & & \\
\hline $16510-0836$ & RST3960 & 2011.521 & 1 & 1.211 & 0.018 & 108.3 & 0.8 & & & & & \\
\hline $16511+0924$ & STF2106 & 2011.510 & 1 & 0.752 & 0.007 & $173.7^{*}$ & 0.3 & & & Sca2001g & -0.02 & 1.7 \\
\hline $16518+2840$ & STF2107 & 2012.496 & 1 & 1.433 & 0.007 & $102.2^{*}$ & 0.3 & & & Izm2019 & 0.00 & -0.6 \\
\hline $16519+6755$ & HEI386 & 2011.518 & 1 & 0.101 & 0.055 & 171.5 & 8.2 & & & & & \\
\hline $16521+6746$ & MLR185 & 2011.515 & 1 & - & - & - & - & & NR & & & \\
\hline $16521+6746$ & MLR185 & 2012.523 & 1 & - & - & - & - & & NR & & & \\
\hline $16522+1535$ & HU1278 & 2011.524 & 1 & 1.328 & 0.010 & $0.2^{*}$ & 0.3 & & & & & \\
\hline $16524+3521$ & COU987 & 2012.487 & 1 & 1.528 & 0.008 & $27.4^{*}$ & 0.3 & & & & & \\
\hline $16533+4122$ & COU1286 & 2011.455 & 1 & 0.866 & 0.007 & $136.7^{*}$ & 0.3 & & & & & \\
\hline
\end{tabular}




\begin{tabular}{|c|c|c|c|c|c|c|c|c|c|c|c|c|}
\hline WDS & Name & Epoch & Bin. & $\begin{array}{c}\rho \\
(")\end{array}$ & $\begin{array}{l}\sigma_{\rho} \\
(")\end{array}$ & $\begin{array}{c}\theta \\
\left({ }^{\circ}\right)\end{array}$ & $\begin{array}{l}\sigma_{\theta} \\
\left(^{\circ}\right)\end{array}$ & $\Delta m$ & Notes & Orbit & $\begin{array}{r}\Delta \rho(\mathrm{O}-\mathrm{C}) \\
(")\end{array}$ & $\begin{array}{r}\Delta \theta(\mathrm{O}-\mathrm{C}) \\
\left({ }^{\circ}\right)\end{array}$ \\
\hline $16539+2547$ & COU492 & 2012.547 & 1 & 0.536 & 0.012 & $92.0^{*}$ & 0.3 & & & & & \\
\hline $16540+2906$ & A350 & 2012.517 & 1 & 0.593 & 0.007 & $148.0^{*}$ & * 0.4 & & & & & \\
\hline $16544+5802$ & MLR431 & 2012.523 & 1 & 2.220 & 0.011 & $9.9^{*}$ & 0.3 & & & & & \\
\hline $16550+3223$ & COU988 & 2011.455 & 1 & 1.640 & 0.010 & $265.6^{*}$ & $* 0.3$ & & & & & \\
\hline $16557+1544$ & HEI70 & 2011.510 & 1 & 1.303 & 0.013 & 137.6 & 0.3 & & & & & \\
\hline $16557+3511$ & RUC9 & 2012.487 & 1 & - & - & - & - & & NR & & & \\
\hline $16564+3755$ & COU1287 & 2012.479 & 1 & 0.719 & 0.007 & $162.6^{*}$ & * 0.3 & & & & & \\
\hline $16564+6502$ & STF2118 & 2011.518 & 1 & 1.031 & 0.007 & 66.6 & 0.3 & & & Izm2019 & 0.00 & 0.4 \\
\hline $16564+6502$ & STF2118 & 2012.531 & 1 & 1.023 & 0.007 & $66.5^{*}$ & 0.3 & & & Izm2019 & 0.01 & 0.4 \\
\hline $16564+6502$ & STF2118 & 2012.531 & 1 & 1.014 & 0.017 & 66.9 & 0.3 & & & Izm2019 & -0.00 & 0.8 \\
\hline $16565+4108$ & COU1288 & 2011.455 & 1 & 1.503 & 0.008 & $182.8^{*}$ & * 0.3 & & & & & \\
\hline $16566+5711$ & A1143AB & 2011.518 & 1 & 0.317 & 0.008 & 46.1 & 0.9 & & & Hrt2000c & -0.01 & $-2.0^{Q}$ \\
\hline $16566+5711$ & A1143AB & 2012.531 & 1 & 0.327 & 0.007 & $45.4^{*}$ & 0.5 & & & Hrt2000c & 0.00 & $-0.8^{Q}$ \\
\hline $16566+5711$ & A1143AB & 2012.531 & 1 & 0.345 & 0.017 & 47.0 & 0.6 & & & Hrt2000c & 0.02 & $0.8^{Q}$ \\
\hline $16566+5711$ & A1143CD & 2011.518 & 1 & 0.713 & 0.007 & $139.8^{*}$ & $* 0.3$ & & & & & \\
\hline $16566+5711$ & A1143CD & 2012.531 & 1 & 0.693 & 0.008 & 137.7 & 1.4 & & & & & \\
\hline $16566+5711$ & A1143CD & 2012.531 & 1 & 0.696 & 0.007 & $139.6^{*}$ & * 0.4 & & & & & \\
\hline $16567+1539$ & HDS2395 & 2011.526 & 1 & 0.148 & 0.040 & 153.1 & 8.0 & & & & & \\
\hline $16569+1540$ & HEI71AB & 2011.510 & 1 & 1.267 & 0.008 & $76.2^{*}$ & 0.3 & & & & & \\
\hline $16573+4950$ & COU1771 & 2012.517 & 1 & 1.815 & 0.009 & $243.8^{*}$ & * 0.3 & & & & & \\
\hline $16574+1444$ & A1142 & 2011.510 & 1 & 2.045 & 0.010 & $129.2^{*}$ & * 0.3 & & & & & \\
\hline $16576+6027$ & HDS2398 & 2011.518 & 1 & 0.466 & 0.010 & $59.5^{*}$ & 0.3 & & & & & \\
\hline $16579-0644$ & RST3964 & 2011.521 & 1 & 2.399 & 0.015 & $184.0^{*}$ & * 0.3 & & & & & \\
\hline $16583+3107$ & COU989 & 2012.487 & 1 & 0.266 & 0.007 & $194.6^{*}$ & $* 0.7$ & & & & & \\
\hline $16584+3943$ & COU1289 & 2011.469 & 1 & - & - & - & - & & NR & & & \\
\hline $16584+3943$ & COU1289 & 2012.479 & 1 & 0.131 & 0.040 & 63.1 & 8.0 & & & Doc2013e & 0.02 & -3.3 \\
\hline $16584+6935$ & WRH25AB & 2012.523 & 1 & 0.174 & 0.040 & 174.2 & 8.0 & & & & & \\
\hline $16584+6935$ & WRH25AC & 2011.518 & 1 & 1.244 & 0.008 & $343.5^{*}$ & * 0.6 & & & & & \\
\hline $16584+6935$ & WRH25AC & 2012.523 & 1 & 1.294 & 0.008 & $342.9^{*}$ & * 0.3 & & & & & \\
\hline $17003+0106$ & $\mathrm{~A} 2235$ & 2011.521 & 1 & 0.801 & 0.007 & $267.0^{*}$ & * 0.3 & & & & & \\
\hline $17005+0635$ & CHR59 & 2011.526 & 1 & - & - & - & - & & NR & & & \\
\hline $17005+7514$ & HDS2408 & 2011.518 & 1 & 0.601 & 0.007 & $110.8^{*}$ & * 0.4 & & & & & \\
\hline $17006+2002$ & COU199AB & 2011.556 & 1 & 0.244 & 0.018 & 13.5 & 4.4 & & & & & \\
\hline $17006+2002$ & COU199AC & 2011.556 & 1 & 1.300 & 0.007 & $331.4^{*}$ & * 0.4 & & ND & & & \\
\hline $17006+2026$ & COU200 & 2012.487 & 1 & 1.465 & 0.010 & $132.8^{*}$ & * 0.3 & & & & & \\
\hline $17006+2026$ & COU200 & 2011.455 & 1 & 1.464 & 0.009 & $312.7^{*}$ & $* 0.3$ & & & & & \\
\hline $17013+6937$ & HEI245 & 2012.528 & 1 & 1.353 & 0.013 & 296.2 & 1.4 & & & & & \\
\hline $17013+6937$ & HEI245 & 2012.528 & 1 & 1.362 & 0.015 & $296.1^{*}$ & * 0.5 & & & & & \\
\hline $17014-0047$ & RST5073 & 2011.521 & 1 & 0.675 & 0.009 & $21.1^{*}$ & 0.9 & & & & & \\
\hline $17019+3943$ & BAG26 & 2012.575 & 1 & 0.287 & 0.021 & 31.8 & 2.2 & & & & & \\
\hline $17036+6948$ & A1146 & 2011.515 & 1 & 0.325 & 0.012 & $104.2^{*}$ & * 0.8 & & & USN2007b & 0.03 & -2.5 \\
\hline $17039+1941$ & BU822 & 2011.524 & 1 & 1.528 & 0.008 & $223.9^{*}$ & * 0.3 & & & & & \\
\hline $17039+3212$ & DAE6 & 2012.575 & 1 & 1.365 & 0.010 & 149.0 & 0.9 & 0.65 & & & & \\
\hline $17039+3212$ & DAE6 & 2012.575 & 1 & 1.341 & 0.036 & 147.4 & 0.7 & & & & & \\
\hline $17039+3212$ & DAE6 & 2012.575 & 1 & 1.343 & 0.007 & 147.1 & 0.6 & & & & & \\
\hline $17039+3212$ & DAE6 & 2012.586 & 1 & 1.339 & 0.027 & 146.7 & 0.5 & 2.23 & & & & \\
\hline $17039+3212$ & DAE6 & 2012.586 & 1 & 1.341 & 0.007 & $147.8^{*}$ & * 0.3 & & & & & \\
\hline $17039+3212$ & DAE6 & 2012.586 & 1 & 1.353 & 0.008 & 148.1 & 0.3 & & & & & \\
\hline $17039+5314$ & MLR603 & 2012.528 & 1 & 0.368 & 0.013 & 166.8 & 1.2 & & & & & \\
\hline $17043+4445$ & COU1593 & 2012.517 & 1 & 0.490 & 0.007 & $199.1^{*}$ & $* 0.3$ & & & & & \\
\hline $17043+4445$ & COU1593 & 2011.469 & 1 & 0.490 & 0.007 & $198.0^{*}$ & * 0.5 & & & & & \\
\hline $17044+3314$ & HO555 & 2011.556 & 1 & 1.462 & 0.012 & $186.3^{*}$ & $* 0.7$ & & & & & \\
\hline $17046-0339$ & RST4565 & 2011.521 & 1 & 0.779 & 0.009 & $165.3^{*}$ & $* 0.3$ & & & & & \\
\hline $17047+1936$ & PRY2AB & 2011.573 & 1 & 1.849 & 0.009 & 47.7 & 0.3 & & & & & \\
\hline
\end{tabular}




\begin{tabular}{|c|c|c|c|c|c|c|c|c|c|c|c|c|}
\hline WDS & Name & Epoch & Bin. & $\begin{array}{c}\rho \\
(")\end{array}$ & $\begin{array}{l}\sigma_{\rho} \\
(")\end{array}$ & $\begin{array}{c}\theta \\
\left({ }^{\circ}\right)\end{array}$ & $\begin{array}{l}\sigma_{\theta} \\
\left(^{\circ}\right)\end{array}$ & $\Delta m$ & Notes & Orbit & $\begin{array}{r}\Delta \rho(\mathrm{O}-\mathrm{C}) \\
(")\end{array}$ & $\begin{array}{r}\Delta \theta(\mathrm{O}-\mathrm{C}) \\
\left({ }^{\circ}\right)\end{array}$ \\
\hline $17051+1353$ & HDS2417 & 2011.526 & 1 & - & - & - & - & & NR & & & \\
\hline $17053+5927$ & MLR353 & 2011.518 & 1 & 0.718 & 0.007 & $353.5^{*}$ & 0.3 & & & & & \\
\hline $17055+1033$ & BU357 & 2011.524 & 1 & 1.457 & 0.008 & $307.2^{*}$ & 0.3 & & & & & \\
\hline $17060+4616$ & TDT212 & 2012.547 & 1 & 1.209 & 0.007 & $131.0^{*}$ & 0.3 & & & & & \\
\hline $17063+2631$ & $\mathrm{~A} 228$ & 2012.569 & 1 & 0.534 & 0.007 & $6.9^{*}$ & 0.8 & & & & & \\
\hline $17063+2631$ & A228 & 2012.569 & 1 & 0.537 & 0.015 & 7.2 & 0.5 & 0.55 & & & & \\
\hline $17063+2631$ & A228 & 2012.569 & 1 & 0.511 & 0.010 & 4.7 & 2.2 & & & & & \\
\hline $17064+3114$ & TDT214 & 2012.548 & 1 & 0.734 & 0.007 & $354.9^{*}$ & 0.7 & & & & & \\
\hline $17067-0749$ & RST3965 & 2011.521 & 1 & 1.683 & 0.008 & $296.8^{*}$ & 0.3 & & & & & \\
\hline $17067+2225$ & COU110 & 2011.556 & 1 & 1.430 & 0.007 & $90.6^{*}$ & 0.3 & & & & & \\
\hline $17073+4604$ & COU1773 & 2011.469 & 1 & 0.914 & 0.007 & $23.7^{*}$ & 0.3 & & & & & \\
\hline $17073+5920$ & MLR354 & 2011.518 & 1 & 1.675 & 0.008 & $288.8^{*}$ & 0.3 & & & & & \\
\hline $17074+6229$ & HU918 & 2011.515 & 1 & 0.233 & 0.012 & 37.6 & 1.8 & & & & & \\
\hline $17075+3810$ & COU1291 & 2011.469 & 1 & 0.238 & 0.007 & $126.3^{*}$ & 1.2 & & & Doc2011a & -0.04 & $1.9^{Q}$ \\
\hline $17075+3810$ & COU1291 & 2011.469 & 1 & 0.272 & 0.008 & 125.3 & 1.7 & & & Doc2011a & -0.01 & $0.9^{Q}$ \\
\hline $17075+3810$ & COU1291 & 2012.479 & 1 & 0.274 & 0.007 & $127.6^{*}$ & 1.8 & & & Doc2011a & -0.01 & $2.0^{Q}$ \\
\hline $17075+5126$ & COU1921 & 2012.496 & 1 & 0.528 & 0.007 & $284.8^{*}$ & 0.4 & & & & & \\
\hline $17076+4108$ & COU1292 & 2011.455 & 1 & 0.523 & 0.007 & $347.8^{*}$ & 0.7 & & & & & \\
\hline $17080-0957$ & RST3966 & 2011.521 & 1 & 0.442 & 0.010 & $108.7^{*}$ & 0.9 & & & & & \\
\hline $17080+3556$ & HU1176 & 2011.559 & 1 & 0.173 & 0.040 & $99.8^{*}$ & 8.0 & & & Mut2010b & 0.04 & -1.1 \\
\hline $17080+3556$ & HU1176 & 2012.517 & 1 & 0.114 & 0.040 & 98.1 & 8.0 & & & Mut2010b & 0.01 & 12.5 \\
\hline $17083+5051$ & CHR60Aa,Ab & 2012.520 & 1 & - & - & - & - & & NR & & & \\
\hline $17084-0518$ & RST4566 & 2011.526 & 1 & 0.256 & 0.008 & $188.9^{*}$ & 0.6 & & & & & \\
\hline $17084+3229$ & TDT227 & 2012.548 & 1 & - & - & - & - & & NR & & & \\
\hline $17087+1945$ & $\mathrm{~J} 1130$ & 2011.573 & 1 & 1.288 & 0.007 & $278.9^{*}$ & 0.4 & & & & & \\
\hline $17088+4856$ & COU1774 & 2011.559 & 1 & 0.583 & 0.013 & $97.6^{*}$ & 0.9 & & & & & \\
\hline $17094+2423$ & A 229 & 2012.520 & 1 & 1.213 & 0.007 & $356.9^{*}$ & 0.3 & & & & & \\
\hline $17094+2423$ & A229 & 2012.520 & 1 & 1.210 & 0.007 & $356.8^{*}$ & 0.3 & & & & & \\
\hline $17100+6812$ & MLR200 & 2011.518 & 1 & 1.153 & 0.007 & $90.2^{*}$ & 0.3 & & & & & \\
\hline $17102-0046$ & BU124 & 2011.524 & 1 & 0.948 & 0.017 & $261.3^{*}$ & 0.3 & & & & & \\
\hline $17103+5403$ & HDS2424 & 2012.523 & 1 & 0.518 & 0.008 & 157.6 & 0.3 & & & & & \\
\hline $17105+4645$ & A1645 & 2012.520 & 1 & 0.246 & 0.007 & $219.0^{*}$ & 0.4 & & & & & \\
\hline $17105+4645$ & A1645 & 2012.520 & 1 & 0.233 & 0.007 & $36.8^{*}$ & 0.6 & & & & & \\
\hline $17107+1651$ & HEI167 & 2011.510 & 1 & 0.386 & 0.007 & $93.2^{*}$ & 0.7 & & & & & \\
\hline $17107+2104$ & TDT251 & 2012.548 & 1 & 0.606 & 0.012 & 117.9 & 0.9 & & & & & \\
\hline $17109+4044$ & COU1293 & 2012.517 & 1 & 0.203 & 0.011 & 9.4 & 0.4 & & & & & \\
\hline $17110+1622$ & HEI168 & 2011.510 & 1 & 0.760 & 0.007 & 65.4 & 0.4 & & & & & \\
\hline $17115-0956$ & HDS2428 & 2011.526 & 1 & 0.322 & 0.007 & $348.5^{*}$ & 2.2 & & & & & \\
\hline $17115+4914$ & COU1775 & 2012.548 & 1 & 0.165 & 0.040 & 6.0 & 8.0 & & & & & \\
\hline $17116+3916$ & HU1178AB & 2012.487 & 1 & 0.340 & 0.007 & $10.2^{*}$ & 0.3 & & & Zir2012 & -0.01 & 1.0 \\
\hline $17116+3916$ & HU1178CD & 2012.487 & 1 & 1.381 & 0.007 & $76.6^{*}$ & 0.3 & & & & & \\
\hline $17121+2515$ & COU313 & 2012.569 & 1 & 0.793 & 0.007 & 153.9 & 0.5 & & & & & \\
\hline $17121+2515$ & COU313 & 2012.569 & 1 & 0.784 & 0.015 & 152.9 & 1.3 & & & & & \\
\hline $17121+4540$ & KUI79 & 2012.487 & 1 & 1.103 & 0.047 & $224.3^{*}$ & 0.3 & & & Hrt1996a & 0.02 & -0.5 \\
\hline $17121+4540$ & KUI79 & 2011.455 & 1 & 1.137 & 0.047 & $232.0^{*}$ & 0.3 & & & Hrt1996a & 0.00 & -0.5 \\
\hline $17125+2802$ & COU493 & 2012.496 & 1 & 0.295 & 0.010 & $55.5^{*}$ & 1.5 & & & & & \\
\hline $17129-0216$ & RST4568 & 2011.521 & 1 & 1.599 & 0.015 & $318.7^{*}$ & 0.5 & & & & & \\
\hline $17134+4944$ & COU1776 & 2011.559 & 1 & 0.418 & 0.007 & $96.7^{*}$ & 0.3 & & & & & \\
\hline $17134+4944$ & COU1776 & 2012.517 & 1 & 0.462 & 0.007 & $276.7^{*}$ & 0.3 & & & & & \\
\hline $17136+0405$ & HEI895 & 2011.510 & 1 & 0.418 & 0.007 & 17.3 & 2.1 & & & & & \\
\hline $17136+1716$ & A2087 & 2012.496 & 1 & 0.435 & 0.007 & $131.2^{*}$ & 0.3 & & & Mnt2001a & -0.01 & -2.1 \\
\hline $17141+5608$ & STT327 & 2012.531 & 1 & 0.461 & 0.007 & $154.6^{*}$ & 0.5 & & & Msn2011c & 0.01 & $1.4^{Q}$ \\
\hline $17141+5608$ & STT327 & 2012.531 & 1 & 0.461 & 0.007 & 153.8 & 0.6 & & & Msn2011c & 0.01 & $1.4^{Q}$ \\
\hline $17142+0622$ & $\mathrm{~A} 1147 \mathrm{AB}$ & 2011.526 & 1 & 0.186 & 0.040 & 181.0 & 8.0 & & & & & \\
\hline
\end{tabular}




\begin{tabular}{|c|c|c|c|c|c|c|c|c|c|c|c|c|}
\hline WDS & Name & Epoch & Bin. & $\begin{array}{c}\rho \\
(")\end{array}$ & $\begin{array}{l}\sigma_{\rho} \\
(")\end{array}$ & $\begin{array}{c}\theta \\
\left({ }^{\circ}\right)\end{array}$ & $\begin{array}{l}\sigma_{\theta} \\
\left(^{\circ}\right)\end{array}$ & $\Delta m$ & Notes & Orbit & $\begin{array}{r}\Delta \rho(\mathrm{O}-\mathrm{C}) \\
(")\end{array}$ & $\begin{array}{r}\Delta \theta(\mathrm{O}-\mathrm{C}) \\
\left({ }^{\circ}\right)\end{array}$ \\
\hline $17142+0622$ & $\mathrm{~A} 1147 \mathrm{AB}, \mathrm{C}$ & 2011.526 & 1 & 5.328 & 0.027 & 1139.1 & 0.3 & & & & & \\
\hline $17142+2731$ & COU495 & 2011.455 & 1 & 0.784 & 0.007 & $280.8^{*}$ & 0.4 & & & & & \\
\hline $17145+4331$ & HDS2437 & 2011.469 & 1 & 0.364 & 0.007 & 31.9 & 1.2 & & & & & \\
\hline $17151+6639$ & MLR186 & 2011.515 & 1 & 0.224 & 0.022 & 25.8 & 4.3 & & & & & \\
\hline $17151+6639$ & MLR186 & 2012.523 & 1 & - & - & - & - & & NR & & & \\
\hline $17152+3214$ & COU805 & 2012.518 & 1 & 0.858 & 0.007 & $235.3^{*}$ & 0.5 & & & & & \\
\hline $17155+1052$ & HDS2440 & 2011.526 & 1 & 0.131 & 0.059 & 192.9 & 9.1 & & & Cve2014 & 0.03 & 2.9 \\
\hline $17157-0949$ & A2592 & 2011.521 & 1 & 0.169 & 0.040 & 136.9 & 6.0 & & & Tok2015c & -0.01 & -0.6 \\
\hline $17158+4016$ & COU1294 & 2011.469 & 1 & 0.978 & 0.007 & 83.3 & 0.3 & & & & & \\
\hline $17161+2316$ & COU315 & 2011.455 & 1 & 0.148 & 0.040 & 135.6 & 8.0 & & & Doc2020b & 0.07 & 14.7 \\
\hline $17161+2316$ & COU315 & 2012.518 & 1 & 0.121 & 0.040 & 128.2 & 8.0 & & & Doc2020b & 0.05 & 17.8 \\
\hline $17163+3458$ & COU1141 & 2011.469 & 1 & 0.497 & 0.007 & 138.1 & 1.0 & & & & & \\
\hline $17163+3458$ & COU1141 & 2012.479 & 1 & 0.508 & 0.009 & $138.8^{*}$ & 0.5 & & & & & \\
\hline $17164+4643$ & HDS2442 & 2011.559 & 1 & 0.616 & 0.007 & $54.6^{*}$ & 0.7 & & & & & \\
\hline $17164+4643$ & HDS2442 & 2012.589 & 1 & 0.607 & 0.007 & $52.4^{*}$ & 0.7 & & & & & \\
\hline $17166+1440$ & BU1200 & 2011.526 & 1 & - & - & - & - & & NR & & & \\
\hline $17166+2635$ & WHC15 & 2012.520 & 1 & 0.125 & 0.042 & $63.3^{*}$ & 6.7 & & & & & \\
\hline $17166+7136$ & HDS2443 & 2011.518 & 1 & 0.573 & 0.007 & $141.4^{*}$ & 0.7 & & & & & \\
\hline $17171+4034$ & COU1295 & 2011.469 & 1 & 0.252 & 0.009 & 55.5 & 0.5 & & & & & \\
\hline $17173+2113$ & HU668 & 2012.583 & 1 & 1.331 & 0.007 & 37.6 & 0.4 & & & & & \\
\hline $17174+1113$ & HU172 & 2011.526 & 1 & 1.131 & 0.007 & $344.9^{*}$ & 0.3 & & & & & \\
\hline $17179+4918$ & STF2153 & 2012.569 & 1 & 1.465 & 0.011 & 64.8 & 0.7 & & & Izm2019 & -0.01 & -0.8 \\
\hline $17179+4918$ & STF2153 & 2012.569 & 1 & 1.468 & 0.007 & 66.2 & 0.7 & 1.62 & & Izm2019 & -0.00 & 0.6 \\
\hline $17181+2742$ & A231 & 2012.575 & 1 & - & - & - & - & & NR & & & \\
\hline $17182+1559$ & HEI246 & 2011.573 & 1 & 1.120 & 0.007 & $42.4^{*}$ & 0.3 & & & & & \\
\hline $17182+4952$ & HU669 & 2012.569 & 1 & 0.897 & 0.009 & 80.7 & 1.4 & & & & & \\
\hline $17182+4952$ & HU669 & 2012.569 & 1 & 0.888 & 0.010 & 82.7 & 1.0 & 0.36 & & & & \\
\hline $17184+3240$ & BU628 & 2012.548 & 1 & 0.548 & 0.007 & $263.8^{*}$ & 0.3 & & & FMR2012a & 0.00 & -1.4 \\
\hline $17193+4917$ & HU670 & 2012.548 & 1 & 0.192 & 0.040 & $18.8^{*}$ & 8.0 & & & & & \\
\hline $17194+2802$ & COU625 & 2012.496 & 1 & 0.129 & 0.052 & 99.4 & 8.0 & & & & & \\
\hline $17199+1807$ & COU2625 & 2011.573 & 1 & 2.344 & 0.016 & $8.7^{*}$ & 0.3 & & & & & \\
\hline $17201+6206$ & HU920 & 2011.518 & 1 & 0.457 & 0.007 & $312.6^{*}$ & 0.9 & & & & & \\
\hline $17210-0014$ & RST5079 & 2011.526 & 1 & 1.294 & 0.007 & 62.1 & 0.3 & & & & & \\
\hline $17215+2845$ & KUI80 & 2012.520 & 1 & 0.634 & 0.010 & $173.2^{*}$ & 1.1 & & & & & \\
\hline $17215+2845$ & KUI80 & 2011.469 & 1 & 0.639 & 0.013 & $171.5^{*}$ & 0.3 & & & & & \\
\hline $17220+5351$ & BU1249 & 2012.523 & 1 & 0.543 & 0.015 & 265.5 & 2.1 & & & & & \\
\hline $17220+5351$ & BU1249 & 2012.523 & 1 & 0.577 & 0.007 & 260.8 & 1.3 & & & & & \\
\hline $17220+5351$ & BU1249 & 2012.523 & 1 & 0.586 & 0.007 & $262.4^{*}$ & 0.3 & & & & & \\
\hline $17220+5351$ & BU1249 & 2012.523 & 1 & 0.586 & 0.007 & 259.5 & 2.5 & & & & & \\
\hline $17221+2310$ & COU415 & 2011.456 & 1 & 0.204 & 0.010 & $241.7^{*}$ & 4.6 & & & Msn2011c & -0.05 & -4.1 \\
\hline $17221+2310$ & COU415 & 2012.496 & 1 & 0.218 & 0.007 & $246.2^{*}$ & 1.5 & & & Msn2011c & -0.04 & 4.2 \\
\hline $17226+3037$ & TDT325 & 2012.575 & 1 & 0.184 & 0.040 & $125.9^{*}$ & 8.0 & & & & & \\
\hline $17227+2808$ & TDT326 & 2012.548 & 1 & 0.812 & 0.007 & $69.4^{*}$ & 0.6 & & & & & \\
\hline $17228-0344$ & HDS2453 & 2011.526 & 1 & - & - & - & - & & NR & & & \\
\hline $17229+4723$ & COU1777 & 2012.569 & 1 & 0.444 & 0.021 & 42.4 & 1.5 & & & & & \\
\hline $17229+4723$ & COU1777 & 2012.569 & 1 & 0.425 & 0.027 & 44.1 & 1.5 & 0.08 & & & & \\
\hline $17231+2545$ & $\mathrm{HO} 415$ & 2012.583 & 1 & 0.890 & 0.007 & $344.1^{*}$ & 0.3 & & & & & \\
\hline $17234+4209$ & STF2163 & 2011.573 & 1 & 1.427 & 0.009 & $76.9^{*}$ & 0.3 & & & & & \\
\hline $17235+1654$ & A2183 & 2011.573 & 1 & 1.182 & 0.007 & $135.9^{*}$ & 0.3 & & & & & \\
\hline $17239+3627$ & STF2162 & 2011.556 & 1 & 1.345 & 0.007 & $284.8^{*}$ & 0.3 & & & & & \\
\hline $17240-0921$ & RST3972Aa,Ab & 2011.526 & 1 & 0.224 & 0.007 & $250.3^{*}$ & 1.5 & & & Sod1999 & 0.00 & -0.7 \\
\hline $17240+3835$ & HU1179 & 2012.575 & 1 & 0.266 & 0.010 & 276.0 & 0.7 & & & Hrt2000b & 0.00 & 5.3 \\
\hline $17246+3913$ & WSI2Aa,Ab & 2011.469 & 1 & 0.205 & 0.047 & 80.3 & 5.6 & & & & & \\
\hline
\end{tabular}




\begin{tabular}{|c|c|c|c|c|c|c|c|c|c|c|c|c|}
\hline WDS & Name & Epoch & Bin. & $\begin{array}{c}\rho \\
(")\end{array}$ & $\begin{array}{l}\sigma_{\rho} \\
(")\end{array}$ & $\begin{array}{c}\theta \\
\left({ }^{\circ}\right)\end{array}$ & $\begin{array}{l}\sigma_{\theta} \\
\left({ }^{\circ}\right)\end{array}$ & $\Delta m$ & Notes & Orbit & $\begin{array}{r}\Delta \rho(\mathrm{O}-\mathrm{C}) \\
(")\end{array}$ & $\begin{array}{r}\Delta \theta(\mathrm{O}-\mathrm{C}) \\
\left({ }^{\circ}\right)\end{array}$ \\
\hline $17246+3913$ & WSI2Aa,Ab & 2011.469 & 1 & 0.344 & 0.039 & 79.01 & 2.2 & & & & & \\
\hline $17246+3913$ & WSI2Aa,Ab & 2012.569 & 1 & 0.362 & 0.038 & 73.5 & 1.9 & 1.61 & & & & \\
\hline $17247+3802$ & HSL1Aa,Ac & 2011.556 & 1 & 0.168 & 0.040 & 43.8 & 8.0 & & & & & \\
\hline $17247+3802$ & COU1142 & 2011.556 & 1 & 1.939 & 0.010 & 41.5 & 0.3 & & & & & \\
\hline $17248+3044$ & BU1250 & 2012.520 & 1 & 1.981 & 0.010 & $117.2^{*}$ & 0.3 & & & Izm2019 & 0.07 & -0.9 \\
\hline $17250+4306$ & COU1454 & 2011.573 & 1 & 0.972 & 0.007 & $327.8^{*}$ & 0.3 & & & & & \\
\hline $17251+3444$ & HU922Aa,Ab & 2012.479 & 1 & 0.252 & 0.007 & $39.9^{*}$ & 1.7 & & & FMR2016b & 0.00 & 0.8 \\
\hline $17255+0030$ & RST5432 & 2011.510 & 1 & 0.236 & 0.007 & 187.1 & 0.9 & & & Hrt2009 & 0.02 & -6.8 \\
\hline $17256+4145$ & COU1296 & 2012.487 & 1 & 0.496 & 0.007 & $344.8^{*}$ & 0.8 & & & & & \\
\hline $17263+6746$ & BU1201 & 2011.515 & 1 & 0.126 & 0.057 & $341.3^{*}$ & 5.4 & & & & & \\
\hline $17266+7331$ & MLR201 & 2011.515 & 1 & 0.424 & 0.009 & $258.9^{*}$ & 1.2 & & & & & \\
\hline $17268+2625$ & COU1455 & 2012.569 & 1 & 0.239 & 0.035 & 178.3 & 1.2 & 0.77 & & & & \\
\hline $17271+2402$ & TDT360 & 2012.000 & 2 & 0.677 & 0.060 & 178.6 & 1.3 & & & & & \\
\hline $17271+2402$ & TDT360 & 2012.589 & 1 & 0.702 & 0.057 & $178.0^{*}$ & 0.4 & & & & & \\
\hline $17271+2402$ & TDT360 & 2012.589 & 1 & 0.655 & 0.041 & 182.0 & 1.7 & & & & & \\
\hline $17272+2250$ & TDT363 & 2012.548 & 1 & 1.544 & 0.008 & $190.1^{*}$ & 0.3 & & & & & \\
\hline $17276+2624$ & COU498 & 2012.583 & 1 & 0.455 & 0.007 & $41.2^{*}$ & 0.3 & & & & & \\
\hline $17279+5610$ & A1152 & 2012.523 & 1 & 0.154 & 0.040 & $203.5^{*}$ & 8.0 & & & & & \\
\hline $17279+5610$ & A1152 & 2011.515 & 1 & 0.193 & 0.040 & 201.0 & 8.0 & & & & & \\
\hline $17279+7112$ & A1154 & 2011.518 & 1 & 0.908 & 0.007 & $67.4^{*}$ & 0.3 & & & & & \\
\hline $17285+7430$ & A1155 & 2011.518 & 1 & 0.569 & 0.017 & $286.7^{*}$ & 0.8 & & & & & \\
\hline $17290+3845$ & COU1297 & 2012.479 & 1 & 0.279 & 0.007 & $97.5^{*}$ & 0.5 & & & & & \\
\hline $17292+1543$ & HEI169 & 2011.510 & 1 & 0.650 & 0.011 & 40.4 & 0.3 & & & & & \\
\hline $17296+6953$ & MLR188 & 2012.523 & 1 & 1.848 & 0.017 & $197.5^{*}$ & 0.3 & & & & & \\
\hline $17296+6953$ & MLR188 & 2012.523 & 1 & 1.850 & 0.009 & 197.8 & 0.4 & 0.05 & & & & \\
\hline $17296+6953$ & MLR188 & 2012.523 & 1 & 1.869 & 0.028 & 198.0 & 0.6 & & & & & \\
\hline $17297-0801$ & RST3977 & 2011.526 & 1 & 0.263 & 0.018 & 145.2 & 1.9 & & & & & \\
\hline $17299+1558$ & $\mathrm{HDS} 2471 \mathrm{Aa}, \mathrm{Ab}$ & 2011.469 & 1 & - & - & - & - & & NR & & & \\
\hline $17301+3025$ & TDT383 & 2012.575 & 1 & 1.071 & 0.024 & 242.0 & 1.8 & 2.26 & & & & \\
\hline $17301+3025$ & TDT383 & 2012.575 & 1 & 1.113 & 0.032 & 242.0 & 1.0 & & & & & \\
\hline $17301+3025$ & TDT383 & 2012.575 & 1 & 1.066 & 0.007 & $240.0^{*}$ & 0.3 & & & & & \\
\hline $17308+0349$ & $\mathrm{~A} 2247$ & 2011.510 & 1 & 0.242 & 0.007 & 174.7 & 2.4 & & & & & \\
\hline $17309+1512$ & HDS2473 & 2011.521 & 1 & 0.547 & 0.019 & $142.4^{*}$ & 0.9 & & & & & \\
\hline $17312+4903$ & COU1778 & 2011.559 & 1 & 0.370 & 0.007 & $191.5^{*}$ & 0.3 & & & & & \\
\hline $17313+1901$ & COU499 & 2011.469 & 1 & - & - & - & - & & NR & & & \\
\hline $17313+1901$ & COU499 & 2012.479 & 1 & 0.133 & 0.040 & 348.9 & 8.0 & & & Tok2017c & 0.04 & 1.1 \\
\hline $17318-0055$ & RST5088 & 2011.521 & 1 & 1.161 & 0.008 & 57.9 & 0.4 & & & & & \\
\hline $17321+2310$ & TDT400 & 2012.570 & 1 & 0.455 & 0.007 & 139.8 & 0.3 & & & & & \\
\hline $17321+2310$ & TDT400 & 2012.570 & 1 & 0.459 & 0.016 & 140.7 & 0.9 & 0.37 & & & & \\
\hline $17324+2898$ & $\mathrm{~A} 352 \mathrm{AB}$ & 2011.556 & 1 & - & - & - & - & & NR & & & \\
\hline $17324+2898$ & $\mathrm{~A} 352 \mathrm{AB}$ & 2012.479 & 1 & - & - & - & - & & NR & & & \\
\hline $17326+3445$ & HU1181 & 2012.518 & 1 & 0.150 & 0.040 & 316.7 & 8.0 & & & Sod1999 & 0.05 & -2.2 \\
\hline $17327+0251$ & HDS2475 & 2011.521 & 1 & 0.243 & 0.014 & 31.2 & 2.1 & & & & & \\
\hline $17328+6659$ & HDS2476 & 2011.518 & 1 & 0.422 & 0.010 & $213.1^{*}$ & 0.5 & & & & & \\
\hline $17330+0913$ & HDS2477 & 2011.521 & 1 & - & - & - & - & & NR & & & \\
\hline $17330+2316$ & COU113 & 2012.518 & 1 & 1.241 & 0.007 & $71.0^{*}$ & 0.3 & & & & & \\
\hline $17335+5734$ & MLR571 & 2012.523 & 1 & - & - & - & - & & NR & & & \\
\hline $17343+4657$ & A2090 & 2012.520 & 1 & 0.890 & 0.014 & $112.5^{*}$ & 0.3 & & & & & \\
\hline $17343+4657$ & A2090 & 2012.520 & 1 & 0.877 & 0.007 & $112.7^{*}$ & 0.3 & & & & & \\
\hline $17344+5022$ & COU2113 & 2012.518 & 1 & - & - & - & - & & NR & & & \\
\hline $17344+7801$ & MLR205 & 2011.518 & 1 & 1.142 & 0.007 & 92.2 & 0.3 & & & & & \\
\hline $17345+3935$ & COU1298 & 2012.570 & 1 & 0.268 & 0.069 & 75.8 & 2.6 & & & & & \\
\hline
\end{tabular}




\begin{tabular}{|c|c|c|c|c|c|c|c|c|c|c|c|c|}
\hline WDS & Name & Epoch & Bin. & $\begin{array}{c}\rho \\
(")\end{array}$ & $\begin{array}{l}\sigma_{\rho} \\
(")\end{array}$ & $\begin{array}{c}\theta \\
\left({ }^{\circ}\right)\end{array}$ & $\begin{array}{l}\sigma_{\theta} \\
\left({ }^{\circ}\right)\end{array}$ & $\Delta m$ & Notes & Orbit & $\begin{array}{r}\Delta \rho(\mathrm{O}-\mathrm{C}) \\
(")\end{array}$ & $\begin{array}{r}\Delta \theta(\mathrm{O}-\mathrm{C}) \\
\left({ }^{\circ}\right)\end{array}$ \\
\hline $17345+3935$ & COU1298 & 2012.570 & 1 & 0.322 & 0.063 & 67.3 & 8.4 & 0.67 & & & & \\
\hline $17348+1812$ & TDT422 & 2012.575 & 1 & 0.473 & 0.035 & 138.2 & 0.6 & 0.23 & & & & \\
\hline $17348+1812$ & TDT422 & 2012.575 & 1 & 0.406 & 0.041 & 137.8 & 3.3 & 0.45 & & & & \\
\hline $17348+1812$ & TDT422 & 2012.575 & 1 & 0.500 & 0.007 & 141.1 & 1.5 & & & & & \\
\hline $17348+1812$ & TDT422 & 2012.575 & 1 & 0.486 & 0.009 & $139.9^{*}$ & ${ }^{*} 0.5$ & & & & & \\
\hline $17349+4803$ & HDS2482 & 2011.573 & 1 & 0.204 & 0.037 & 3.4 & 2.7 & & & & & \\
\hline $17349+4803$ & HDS2482 & 2011.573 & 1 & 0.205 & 0.027 & $5.2^{*}$ & 1.5 & & & & & \\
\hline $17349+4803$ & HDS2482 & 2012.518 & 1 & 0.187 & 0.040 & 0.0 & 8.0 & & & & & \\
\hline $17351-0036$ & HDS2484AB & 2011.521 & 1 & 0.792 & 0.010 & $200.9^{*}$ & ${ }^{*} 0.4$ & & & & & \\
\hline $17351-0036$ & HDS2484AC & 2011.521 & 1 & 3.342 & 0.049 & $307.8^{*}$ & ${ }^{*} 0.8$ & & & & & \\
\hline $17354+3443$ & COU995 & 2012.518 & 1 & 0.401 & 0.007 & $332.2^{*}$ & ${ }^{*} 0.3$ & & & & & \\
\hline $17359+3205$ & COU807 & 2012.583 & 1 & 0.646 & 0.007 & $141.1^{*}$ & ${ }^{*} 0.5$ & & & & & \\
\hline $17364+2503$ & COU500 & 2011.456 & 1 & 0.582 & 0.007 & $339.8^{*}$ & ${ }^{*} 0.3$ & & & & & \\
\hline $17365+4142$ & ES636 & 2012.553 & 1 & 1.968 & 0.010 & 127.5 & 0.3 & & & & & \\
\hline $17365+4543$ & COU1595 & 2012.583 & 1 & 0.489 & 0.007 & $265.3^{*}$ & ${ }^{*} 0.4$ & & & & & \\
\hline $17366+4827$ & COU1922 & 2011.456 & 1 & 0.212 & 0.018 & $101.8^{*}$ & * 2.3 & & & Doc2008f & -0.10 & 4.8 \\
\hline $17366+4827$ & COU1922 & 2012.520 & 1 & 0.250 & 0.007 & 94.3 & 0.3 & 2.06 & & Doc2008f & -0.08 & 1.9 \\
\hline $17366+4827$ & COU1922 & 2012.520 & 1 & 0.306 & 0.027 & $91.2^{*}$ & 0.5 & & & Doc2008f & -0.02 & -1.2 \\
\hline $17372+2754$ & KUI83 & 2011.469 & 1 & 0.213 & 0.010 & $265.9^{*}$ & * 1.7 & & & Msn2012a & -0.02 & 1.2 \\
\hline $17372+2754$ & KUI83 & 2012.479 & 1 & 0.228 & 0.007 & $240.3^{*}$ & * 1.7 & & & Msn2012a & -0.01 & -3.5 \\
\hline $17374+1233$ & BU1121 & 2011.521 & 1 & 0.467 & 0.020 & $196.4^{*}$ & ${ }^{*} 0.5$ & & & & & \\
\hline $17377+3017$ & HDS2490 & 2011.573 & 1 & 0.222 & 0.007 & $164.1^{*}$ & ${ }^{*} 0.3$ & & & & & \\
\hline $17380+2842$ & TDS875 & 2012.575 & 1 & 0.863 & 0.007 & 178.7 & 0.7 & & & & & \\
\hline $17380+2842$ & TDS875 & 2012.575 & 1 & 0.869 & 0.011 & 179.3 & 0.3 & 1.58 & & & & \\
\hline $17380+2842$ & TDS875 & 2012.575 & 1 & 0.858 & 0.007 & 179.3 & 0.3 & & & & & \\
\hline $17381+3655$ & HO69 & 2012.605 & 1 & 1.948 & 0.010 & $139.6^{*}$ & ${ }^{*} 0.3$ & & & & & \\
\hline $17386+2219$ & COU316 & 2012.479 & 1 & 2.296 & 0.011 & $276.2^{*}$ & ${ }^{*} 0.3$ & & & & & \\
\hline $17386+5546$ & STF2199 & 2012.531 & 1 & 2.050 & 0.010 & $55.6^{*}$ & 0.3 & & & Izm2019 & 0.03 & 0.2 \\
\hline $17386+5546$ & STF2199 & 2012.531 & 1 & 2.054 & 0.010 & 56.5 & 0.3 & & & Izm2019 & 0.04 & 1.1 \\
\hline $17388+5406$ & HDS2493 & 2012.523 & 1 & 0.524 & 0.007 & $255.3^{*}$ & ${ }^{*} 0.7$ & & & & & \\
\hline $17393+2617$ & COU628 & 2011.456 & 1 & 1.131 & 0.007 & $129.2^{*}$ & ${ }^{*} 0.3$ & & & & & \\
\hline $17393+7020$ & A1372 & 2011.518 & 1 & 1.134 & 0.007 & $82.5^{*}$ & 0.3 & & & & & \\
\hline $17398+4619$ & COU1780 & 2012.520 & 1 & - & - & - & - & & $\mathrm{NR}$ & & & \\
\hline $17403+5047$ & COU2114 & 2012.583 & 1 & 0.366 & 0.007 & $185.8^{*}$ & ${ }^{*} 0.9$ & & & & & \\
\hline $17407+3117$ & HDS2501 & 2011.456 & 1 & 0.786 & 0.007 & 77.8 & 0.5 & & & & & \\
\hline $17417+0109$ & A2249 & 2011.510 & 1 & 1.407 & 0.007 & 123.2 & 0.3 & & & & & \\
\hline $17418+2130$ & COU114 & 2012.518 & 1 & 0.096 & 0.040 & 261.1 & 8.0 & & & Cou1999b & -0.01 & -8.4 \\
\hline $17418+2130$ & COU114 & 2011.456 & 1 & - & - & - & - & & NR & & & \\
\hline $17418+7140$ & MLR202 & 2011.515 & 1 & 0.276 & 0.013 & $112.3^{*}$ & * 1.8 & & & & & \\
\hline $17420+1038$ & HDS2504 & 2011.576 & 1 & 0.323 & 0.015 & 53.1 & 2.2 & & & & & \\
\hline $17420+1557$ & BU1251 & 2011.469 & 1 & 1.428 & 0.007 & $106.3^{*}$ & ${ }^{*} 0.3$ & & & Baz1991a & 0.06 & 2.3 \\
\hline $17421+2103$ & A1371 & 2012.479 & 1 & 0.137 & 0.040 & $136.4^{*}$ & $* 8.0$ & & & & & \\
\hline $17423+3030$ & TDT469 & 2012.589 & 1 & 0.535 & 0.007 & $96.1^{*}$ & 0.3 & & & & & \\
\hline $17425+7456$ & MLR207 & 2011.515 & 1 & 0.196 & 0.040 & 134.9 & 8.0 & & & & & \\
\hline $17425+7456$ & MLR207 & 2011.515 & 1 & 0.182 & 0.040 & 131.8 & 8.0 & & & & & \\
\hline $17430+1215$ & HU1283 & 2011.510 & 1 & 0.452 & 0.007 & $6.1^{*}$ & 0.3 & & & & & \\
\hline $17433+2137$ & DUQ1Aa,Ab & 2011.556 & 1 & - & - & - & - & & $\mathrm{NR}$ & & & \\
\hline $17435+3453$ & COU997 & 2012.548 & 1 & 0.165 & 0.040 & $35.1^{*}$ & 8.0 & & & & & \\
\hline $17436+2237$ & HU1285 & 2011.456 & 1 & 0.542 & 0.007 & $212.4^{*}$ & ${ }^{*} 0.3$ & & & USN2002 & 0.02 & -1.1 \\
\hline $17440+2517$ & TDT482 & 2012.589 & 1 & 0.589 & 0.007 & $18.6^{*}$ & 0.8 & & & & & \\
\hline $17449+6628$ & HU924 & 2012.531 & 1 & 0.338 & 0.021 & 36.9 & 1.3 & & & & & \\
\hline $17449+6628$ & HU924 & 2012.531 & 1 & 0.315 & 0.007 & $33.4^{*}$ & 1.2 & & & & & \\
\hline $17452+2107$ & COU630 & 2011.456 & 1 & 0.190 & 0.066 & 127.8 & 8.0 & & & & & \\
\hline
\end{tabular}




\begin{tabular}{|c|c|c|c|c|c|c|c|c|c|c|c|c|}
\hline WDS & Name & Epoch & Bin. & $\begin{array}{c}\rho \\
(")\end{array}$ & $\begin{array}{l}\sigma_{\rho} \\
(")\end{array}$ & $\begin{array}{c}\theta \\
\left(^{\circ}\right)\end{array}$ & $\begin{array}{l}\sigma_{\theta} \\
\left(^{\circ}\right)\end{array}$ & $\Delta m$ & Notes & Orbit & $\begin{array}{r}\Delta \rho(\mathrm{O}-\mathrm{C}) \\
(")\end{array}$ & $\begin{array}{r}\Delta \theta(\mathrm{O}-\mathrm{C}) \\
\left({ }^{\circ}\right)\end{array}$ \\
\hline $17453+0244$ & HEI798 & 2011.576 & 1 & 1.948 & 0.010 & $103.3^{*}$ & 0.3 & & & & & \\
\hline $17453+1750$ & TDT488 & 2012.589 & 1 & 0.693 & 0.008 & $192.9^{*}$ & 1.0 & & & & & \\
\hline $17455+3554$ & HDS2509 & 2012.548 & 1 & 0.559 & 0.018 & $78.4^{*}$ & 1.4 & & & & & \\
\hline $17456+3032$ & $\mathrm{HO} 70$ & 2012.520 & 1 & 0.305 & 0.007 & $268.5^{*}$ & 0.9 & & & & & \\
\hline $17457+3557$ & TDT493 & 2011.573 & 1 & - & - & - & - & & NR & & & \\
\hline $17462+3853$ & COU1300 & 2011.469 & 1 & 0.753 & 0.007 & $125.7^{*}$ & 0.3 & & & & & \\
\hline $17462+5357$ & HDS2511 & 2012.523 & 1 & 0.469 & 0.010 & 96.5 & 0.8 & & & & & \\
\hline $17462+5357$ & HDS2511 & 2012.523 & 1 & 0.427 & 0.013 & 96.8 & 0.9 & & & & & \\
\hline $17464+3553$ & TDT497 & 2012.575 & 1 & 0.748 & 0.013 & 262.5 & 0.3 & & & & & \\
\hline $17464+3553$ & TDT497 & 2012.575 & 1 & 0.708 & 0.013 & 260.0 & 1.3 & 0.39 & & & & \\
\hline $17464+3553$ & TDT497 & 2012.575 & 1 & 0.723 & 0.007 & $260.9^{*}$ & 0.3 & & & & & \\
\hline $17465+2743$ & $\mathrm{AC} 7 \mathrm{BC}$ & 2011.456 & 1 & 1.070 & 0.007 & $253.1^{*}$ & 0.3 & & & Pru2014 & 0.03 & -0.2 \\
\hline $17465+2743$ & $\mathrm{AC} 7 \mathrm{BC}$ & 2012.479 & 1 & 0.980 & 0.007 & $259.6^{*}$ & 0.3 & & & Pru2014 & 0.03 & -0.3 \\
\hline $17468+0534$ & A1161 & 2011.576 & 1 & 0.785 & 0.007 & $282.9^{*}$ & 0.3 & & & & & \\
\hline $17470+2915$ & TDS881AB & 2012.583 & 1 & 0.171 & 0.040 & 137.8 & 8.0 & & & & & \\
\hline $17470+2915$ & TDS881AC & 2012.583 & 1 & 1.028 & 0.014 & $240.8^{*}$ & 0.3 & & & & & \\
\hline $17471+1742$ & STF2215 & 2011.573 & 1 & 0.498 & 0.007 & $251.6^{*}$ & 0.3 & & & Cve2006e & 0.03 & 1.2 \\
\hline $17471+3235$ & COU634 & 2012.479 & 1 & 0.251 & 0.007 & $83.0^{*}$ & 1.3 & & & & & \\
\hline $17471+4737$ & CHR64 & 2011.573 & 1 & 0.421 & 0.021 & $152.8^{*}$ & 1.7 & & & $\operatorname{Lin} 2012 c$ & 0.01 & 0.6 \\
\hline $17474+7549$ & MLR210 & 2011.518 & 1 & 0.646 & 0.007 & 82.3 & 0.3 & & & & & \\
\hline $17481+0135$ & A 2185 & 2011.510 & 1 & 0.668 & 0.013 & $182.2^{*}$ & 0.3 & & & & & \\
\hline $17484+2738$ & TDT515 & 2012.583 & 1 & - & - & - & - & & NR & & & \\
\hline $17487+3536$ & HU1182 & 2011.573 & 1 & 0.456 & 0.007 & $124.6^{*}$ & 0.3 & & & USN2002 & -0.00 & $-0.8^{Q}$ \\
\hline $17487+3536$ & HU1182 & 2012.570 & 1 & 0.468 & 0.021 & 121.8 & 0.6 & 0.21 & & USN2002 & 0.02 & $-2.5^{Q}$ \\
\hline $17487+3536$ & HU1182 & 2012.570 & 1 & 0.462 & 0.016 & 122.0 & 2.4 & & & USN2002 & 0.01 & $-2.3^{Q}$ \\
\hline $17489-0152$ & BU824 & 2011.526 & 1 & 0.235 & 0.010 & 21.0 & 2.3 & & & & & \\
\hline $17490+2511$ & COU501 & 2011.556 & 1 & 1.917 & 0.010 & $318.8^{*}$ & 0.3 & & & & & \\
\hline $17490+3704$ & COU1145 & 2012.479 & 1 & 0.173 & 0.040 & $124.9^{*}$ & 8.0 & & & Hrt1996a & 0.01 & -1.5 \\
\hline $17494+4028$ & COU1456 & 2011.559 & 1 & 1.377 & 0.013 & $93.5^{*}$ & 0.9 & & & & & \\
\hline $17500+0031$ & A2186 & 2011.510 & 1 & 0.262 & 0.010 & $263.3^{*}$ & 2.2 & & & Zir2013d & 0.03 & 8.5 \\
\hline $17502+2704$ & TDT526 & 2012.586 & 1 & 0.834 & 0.007 & $327.2^{*}$ & 0.3 & & & & & \\
\hline $17503-0335$ & RST4577 & 2011.526 & 1 & 0.270 & 0.011 & $122.2^{*}$ & 0.5 & & & & & \\
\hline $17505+3651$ & COU1146 & 2012.520 & 1 & 0.280 & 0.007 & 149.1 & 0.3 & 4.43 & & & & \\
\hline $17505+4907$ & COU1924 & 2012.518 & 1 & 0.588 & 0.007 & $67.3^{*}$ & 0.3 & & & & & \\
\hline $17507+3638$ & TDS889 & 2012.583 & 1 & 1.349 & 0.007 & 14.9 & 0.3 & & & & & \\
\hline $17507+3638$ & TDS889 & 2012.548 & 1 & 1.355 & 0.007 & $14.2^{*}$ & 0.3 & & & & & \\
\hline $17510+3010$ & COU636 & 2011.556 & 1 & 1.500 & 0.009 & $270.0^{*}$ & 0.6 & & & & & \\
\hline $17512+3821$ & HU1183 & 2012.570 & 1 & 0.472 & 0.013 & 11.4 & 1.8 & 0.94 & & & & \\
\hline $17512+3821$ & HU1183 & 2012.570 & 1 & 0.484 & 0.010 & 10.3 & 2.3 & & & & & \\
\hline $17516+4555$ & A1883 & 2011.573 & 1 & 0.559 & 0.007 & $41.3^{*}$ & 0.4 & & & & & \\
\hline $17523+4057$ & A699 & 2012.605 & 1 & - & - & - & - & & NR & & & \\
\hline $17526+2536$ & A234 & 2012.570 & 1 & 0.125 & 0.042 & 159.5 & 9.8 & & & & & \\
\hline $17528+3408$ & TDT553 & 2012.605 & 1 & 0.695 & 0.007 & $241.1^{*}$ & 0.3 & & & & & \\
\hline $17528+4038$ & TDT552 & 2012.586 & 1 & 1.291 & 0.010 & $224.3^{*}$ & 0.4 & & & & & \\
\hline $17532+3243$ & COU998Aa,Ab & 2011.556 & 1 & 0.261 & 0.010 & 29.7 & 2.0 & & ND & & & \\
\hline $17532+3243$ & COU998Aa,Ab & 2011.576 & 1 & 0.178 & 0.040 & 25.5 & 8.0 & 0.30 & ND & & & \\
\hline $17532+3243$ & COU998Aa,Ab & 2012.479 & 1 & 0.187 & 0.040 & 22.4 & 8.0 & 0.47 & ND & & & \\
\hline $17532+3243$ & COU998Aa,Ab & 2012.518 & 1 & 0.244 & 0.007 & 18.3 & 1.1 & & ND & & & \\
\hline $17532+3243$ & COU998AB & 2011.573 & 1 & 1.601 & 0.010 & $122.0^{*}$ & 0.3 & & & & & \\
\hline $17532+3243$ & COU998AB & 2011.576 & 1 & 1.630 & 0.008 & $122.5^{*}$ & 0.3 & & & & & \\
\hline $17532+3243$ & COU998AB & 2012.479 & 1 & 1.621 & 0.012 & $120.5^{*}$ & 0.5 & & & & & \\
\hline $17532+3243$ & COU998AB & 2012.518 & 1 & 1.645 & 0.008 & $120.6^{*}$ & 0.4 & & & & & \\
\hline $17533+2459$ & A235 & 2011.556 & 1 & 0.179 & 0.040 & 21.0 & 8.0 & & & Hrt2014b & 0.06 & -8.1 \\
\hline
\end{tabular}




\begin{tabular}{|c|c|c|c|c|c|c|c|c|c|c|c|c|}
\hline WDS & Name & Epoch & Bin. & $\begin{array}{c}\rho \\
(")\end{array}$ & $\begin{array}{l}\sigma_{\rho} \\
(")\end{array}$ & $\begin{array}{c}\theta \\
\left({ }^{\circ}\right)\end{array}$ & $\begin{array}{l}\sigma_{\theta} \\
\left(^{\circ}\right)\end{array}$ & $\Delta m$ & Notes & Orbit & $\begin{array}{r}\Delta \rho(\mathrm{O}-\mathrm{C}) \\
(")\end{array}$ & $\begin{array}{r}\Delta \theta(\mathrm{O}-\mathrm{C}) \\
\left({ }^{\circ}\right)\end{array}$ \\
\hline $17533+2459$ & A 235 & 2011.573 & 1 & - & - & - & - & & NR & & & \\
\hline $17533+3605$ & STF2243 & 2012.520 & 1 & 1.126 & 0.007 & $39.0^{*}$ & 0.3 & & & & & \\
\hline $17541+2949$ & $\mathrm{AC} 9$ & 2011.556 & 1 & 1.109 & 0.007 & $240.7^{*}$ & 0.3 & & & & & \\
\hline $17541+4702$ & HDS2525 & 2012.520 & 1 & 0.361 & 0.008 & $184.3^{*}$ & 1.0 & & & & & \\
\hline $17541+4702$ & HDS2525 & 2012.520 & 1 & 0.290 & 0.022 & 186.0 & 2.3 & & & & & \\
\hline $17543+0328$ & A2188 & 2011.510 & 1 & 0.179 & 0.040 & 171.6 & 8.0 & & & & & \\
\hline $17543+5357$ & A1885 & 2012.531 & 1 & 0.407 & 0.007 & $80.5^{*}$ & 0.7 & & & & & \\
\hline $17548+3610$ & COU1303 & 2011.556 & 1 & 1.326 & 0.021 & $75.7^{*}$ & 0.7 & & & & & \\
\hline $17555+4108$ & COU1601 & 2012.589 & 1 & 0.533 & 0.007 & $62.2^{*}$ & 0.3 & & & & & \\
\hline $17556+2508$ & COU503 & 2012.583 & 1 & 0.338 & 0.026 & $114.0^{*}$ & 1.2 & & & & & \\
\hline $17562+3926$ & BU417 & 2011.556 & 1 & 1.010 & 0.007 & $314.4^{*}$ & 0.3 & & & Izm2019 & 0.05 & -0.9 \\
\hline $17565+3139$ & TDT585 & 2012.583 & 1 & 0.557 & 0.007 & 177.2 & 0.3 & & & & & \\
\hline $17571+0004$ & STF2244 & 2011.526 & 1 & 0.638 & 0.007 & $99.3^{*}$ & 0.3 & & & Msn2017e & -0.01 & 0.3 \\
\hline $17572+2400$ & MCA50 & 2011.576 & 1 & - & - & - & - & & NR & & & \\
\hline $17575+3942$ & COU1457 & 2012.520 & 1 & 1.925 & 0.010 & $7.3^{*}$ & 0.3 & & & & & \\
\hline $17575+3942$ & COU1457 & 2012.548 & 1 & 1.915 & 0.010 & $7.2^{*}$ & 0.3 & & & & & \\
\hline $17575+3942$ & COU1457 & 2012.575 & 1 & 1.909 & 0.010 & 6.5 & 0.5 & & & & & \\
\hline $17575+3942$ & COU1457 & 2012.575 & 1 & 1.903 & 0.016 & 7.1 & 0.3 & 1.37 & & & & \\
\hline $17578+0253$ & HDS2532 & 2011.527 & 1 & 0.207 & 0.012 & 5.9 & 1.7 & & & & & \\
\hline $17584+0428$ & KUI84 & 2011.510 & 1 & 0.214 & 0.007 & $6.6^{*}$ & 1.4 & & & Tok2016e & 0.01 & -3.9 \\
\hline $17584+3524$ & COU1000 & 2012.518 & 1 & 0.906 & 0.007 & $155.0^{*}$ & 0.5 & & & & & \\
\hline $17585-0350$ & RST4579 & 2011.527 & 1 & - & - & - & - & & NR & & & \\
\hline $17587+3538$ & COU1002 & 2011.556 & 1 & 0.833 & 0.007 & $165.1^{*}$ & 0.5 & & & & & \\
\hline $17589+2620$ & COU808 & 2011.556 & 1 & 0.939 & 0.012 & $154.6^{*}$ & 0.6 & & & & & \\
\hline $17591+3228$ & HU1185 & 2011.573 & 1 & 0.384 & 0.010 & $141.0^{*}$ & 0.4 & & & Doc $2012 \mathrm{i}$ & 0.01 & 0.3 \\
\hline $17592+3926$ & COU1458 & 2012.518 & 1 & 0.353 & 0.013 & $77.4^{*}$ & 2.6 & & & & & \\
\hline $17593+4902$ & YSC64 & 2012.518 & 1 & 0.528 & 0.009 & 39.4 & 0.7 & & & & & \\
\hline $17593+4902$ & YSC64 & 2012.518 & 1 & 0.512 & 0.007 & 40.6 & 0.3 & 0.65 & & & & \\
\hline $17596+4314$ & COU1784 & 2011.573 & 1 & 2.155 & 0.012 & $97.4^{*}$ & 0.4 & & & & & \\
\hline $17597+2110$ & TDT618 & 2012.632 & 1 & 1.759 & 0.009 & $108.2^{*}$ & 0.3 & & & & & \\
\hline $17599+5304$ & HO563 & 2012.531 & 1 & 0.727 & 0.019 & 190.1 & 1.7 & & & & & \\
\hline $17599+5304$ & HO563 & 2012.531 & 1 & 0.704 & 0.007 & $191.5^{*}$ & 0.3 & & & & & \\
\hline $18000+2449$ & COU115 & 2012.575 & 1 & 0.246 & 0.021 & 116.8 & 1.1 & & & & & \\
\hline $18000+2449$ & COU115 & 2012.575 & 1 & 0.215 & 0.013 & 118.3 & 3.9 & & & & & \\
\hline $18000+6027$ & HDS2537 & 2011.515 & 1 & 0.339 & 0.010 & $267.1^{*}$ & 0.7 & & & & & \\
\hline $18001+2215$ & TDT622 & 2012.583 & 1 & 1.029 & 0.014 & $279.6^{*}$ & 0.4 & & & & & \\
\hline $18003+2154$ & A1374 & 2011.556 & 1 & 0.443 & 0.007 & $220.5^{*}$ & 0.5 & & & Mdz2017 & 0.02 & -1.9 \\
\hline $18003+2154$ & A1374 & 2012.627 & 1 & 0.456 & 0.008 & $217.0^{*}$ & 0.4 & & & Mdz2017 & 0.03 & -3.3 \\
\hline $18007+1736$ & COU810 & 2012.630 & 1 & 0.156 & 0.040 & 108.6 & 8.0 & & & & & \\
\hline $18007+1736$ & COU810 & 2011.510 & 1 & 0.201 & 0.040 & 114.0 & 1.5 & & & & & \\
\hline $18020+1245$ & HDS2538 & 2011.576 & 1 & 0.435 & 0.009 & $213.6^{*}$ & 1.7 & & & & & \\
\hline $18025+4414$ & BU1127Aa,B & 2012.520 & 1 & 0.725 & 0.007 & $48.5^{*}$ & 0.3 & & & Cve2016c & -0.00 & -1.6 \\
\hline $18028+3115$ & TDT651 & 2012.589 & 1 & 0.555 & 0.007 & $46.8^{*}$ & 0.5 & & & & & \\
\hline $18031+2702$ & TDT653 & 2012.575 & 1 & 0.731 & 0.007 & $208.3^{*}$ & 0.3 & & & & & \\
\hline $18031+2702$ & TDT653 & 2012.575 & 1 & 0.711 & 0.026 & 208.5 & 0.7 & 0.49 & & & & \\
\hline $18032+2603$ & HO565 & 2012.630 & 1 & 0.169 & 0.040 & 102.8 & 8.0 & & & & & \\
\hline $18032+2603$ & HO565 & 2012.627 & 1 & 0.198 & 0.040 & $101.3^{*}$ & 8.0 & & & & & \\
\hline $18033+3921$ & STF2275 & 2012.635 & 1 & 0.286 & 0.007 & $120.3^{*}$ & 0.5 & & & Pop2000a & 0.00 & $-2.1^{Q}$ \\
\hline $18035+4032$ & COU1785 & 2012.520 & 1 & 0.145 & 0.040 & 19.4 & 8.0 & & & Doc2008a & 0.01 & -7.1 \\
\hline $18043+4206$ & COU1786Aa,Ab & 2012.518 & 1 & 0.125 & 0.040 & 169.0 & 8.0 & & & Hrt2009 & -0.04 & 2.2 \\
\hline $18047+4650$ & COU2115 & 2012.518 & 1 & 0.339 & 0.011 & $34.4^{*}$ & 1.0 & & & & & \\
\hline $18048+2630$ & TDT666 & 2012.589 & 1 & 0.774 & 0.007 & $33.6^{*}$ & 1.0 & & & & & \\
\hline $18048+5344$ & HDS2546 & 2012.531 & 1 & 0.570 & 0.007 & 235.4 & 0.4 & & & & & \\
\hline $18048+5344$ & HDS2546 & 2012.531 & 1 & 0.613 & 0.007 & $235.2^{*}$ & 0.4 & & & & & \\
\hline
\end{tabular}




\begin{tabular}{|c|c|c|c|c|c|c|c|c|c|c|c|c|}
\hline WDS & Name & Epoch & Bin. & $\begin{array}{c}\rho \\
(")\end{array}$ & $\begin{array}{l}\sigma_{\rho} \\
(")\end{array}$ & $\begin{array}{c}\theta \\
\left({ }^{\circ}\right)\end{array}$ & $\begin{array}{l}\sigma_{\theta} \\
\left(^{\circ}\right)\end{array}$ & $\Delta m$ & Notes & Orbit & $\begin{array}{r}\Delta \rho(\mathrm{O}-\mathrm{C}) \\
(")\end{array}$ & $\begin{array}{r}\Delta \theta(\mathrm{O}-\mathrm{C}) \\
\left({ }^{\circ}\right)\end{array}$ \\
\hline $18053+2357$ & COU116 & 2012.605 & 1 & 0.749 & 0.007 & 69.2 & 0.3 & & & & & \\
\hline $18054+4306$ & COU1787 & 2012.548 & 1 & 0.380 & 0.007 & $325.3^{*}$ & 0.5 & & & & & \\
\hline $18054+4306$ & COU1787 & 2011.573 & 1 & 0.372 & 0.007 & $324.5^{*}$ & 0.9 & & & & & \\
\hline $18058+2127$ & STT341 & 2012.583 & 1 & 0.435 & 0.007 & $92.2^{*}$ & 0.3 & & & Hei1982b & -0.00 & -0.6 \\
\hline $18058+3512$ & TDT678 & 2012.589 & 1 & 0.654 & 0.007 & $234.2^{*}$ & 0.5 & & & & & \\
\hline $18060+3002$ & TDT679 & 2012.635 & 1 & 0.758 & 0.007 & $348.9^{*}$ & 0.4 & & & & & \\
\hline $18063+3824$ & HU1186 & 2012.520 & 1 & 0.196 & 0.040 & $124.4^{*}$ & 8.0 & & & Cve2006c & -0.04 & 4.8 \\
\hline $18064+4437$ & COU1926 & 2012.630 & 1 & 0.292 & 0.015 & $126.8^{*}$ & 1.9 & & & & & \\
\hline $18065+4022$ & STF2282AB & 2012.630 & 1 & 2.580 & 0.013 & $82.7^{*}$ & 0.3 & & & & & \\
\hline $18065+4022$ & STF2282BC & 2012.630 & 1 & 0.208 & 0.010 & $36.3^{*}$ & 1.5 & & & & & \\
\hline $18068+2326$ & COU417 & 2011.556 & 1 & 1.453 & 0.007 & $90.8^{*}$ & 0.3 & & & & & \\
\hline $18070+2824$ & HDS2553 & 2012.635 & 1 & 0.123 & 0.040 & 142.5 & 8.0 & & & & & \\
\hline $18070+3034$ & SCA170 & 2011.557 & 1 & 1.176 & 0.007 & $316.5^{*}$ & 0.3 & & & & & \\
\hline $18070+3034$ & SCA170 & 2012.520 & 1 & 1.192 & 0.007 & 320.4 & 0.3 & 3.09 & & & & \\
\hline $18070+3034$ & SCA170 & 2012.520 & 1 & 1.189 & 0.007 & 320.1 & 0.3 & 3.16 & & & & \\
\hline $18070+3034$ & SCA170 & 2012.520 & 1 & 1.193 & 0.007 & 319.1 & 0.3 & & & & & \\
\hline $18072+2822$ & TDT691 & 2012.583 & 1 & 0.889 & 0.007 & $202.1^{*}$ & 0.3 & & & & & \\
\hline $18072+7131$ & A1168 & 2011.516 & 1 & 0.630 & 0.008 & $353.2^{*}$ & 0.6 & & & & & \\
\hline $18075+1940$ & STT524 & 2012.627 & 1 & 0.470 & 0.007 & $207.1^{*}$ & 0.9 & & & Hrt2000c & 0.01 & -0.7 \\
\hline $18077+0946$ & BU826 & 2011.521 & 1 & 1.038 & 0.007 & 136.8 & 0.9 & & & & & \\
\hline $18078+2606$ & CHR67 & 2011.576 & 1 & 0.360 & 0.008 & 147.1 & 1.9 & & & & & \\
\hline $18083+2941$ & YSC65Aa,Ac & 2012.479 & 1 & - & - & - & - & & NR & & & \\
\hline $18083+2941$ & YSC65Aa,Ac & 2012.479 & 1 & 0.154 & 0.040 & 138.2 & 8.0 & & & & & \\
\hline $18083+2941$ & YSC65Aa,Ac & 2012.518 & 1 & 0.200 & 0.040 & 118.6 & 8.0 & & & & & \\
\hline $18083+2941$ & YSC65Aa,Ac & 2012.630 & 1 & 0.196 & 0.040 & 124.87 & 8.0 & & & & & \\
\hline $18086+1245$ & HDS2556 & 2011.510 & 1 & 0.514 & 0.007 & $124.5^{*}$ & 0.6 & & & & & \\
\hline $18086+1700$ & HDS2555 & 2011.510 & 1 & 0.386 & 0.007 & $113.2^{*}$ & 0.8 & & & & & \\
\hline $18089+2435$ & HDS2558 & 2012.583 & 1 & 0.146 & 0.040 & 139.5 & 8.0 & & & & & \\
\hline $18090+2335$ & HU315 & 2012.548 & 1 & 0.123 & 0.046 & 133.1 & 8.0 & & & & & \\
\hline $18090+2335$ & HU315 & 2012.548 & 1 & 0.123 & 0.046 & 133.1 & 8.0 & & & & & \\
\hline $18092-0817$ & RST3998 & 2011.521 & 1 & 0.311 & 0.007 & $166.2^{*}$ & 0.5 & & & & & \\
\hline $18092+2323$ & TDT711 & 2012.586 & 1 & 0.990 & 0.007 & $293.6^{*}$ & 0.3 & & & & & \\
\hline $18092+3129$ & COU812 & 2012.479 & 1 & 0.639 & 0.007 & $262.9^{*}$ & 0.3 & & & Cou1999b & -0.04 & -19.9 \\
\hline $18092+3129$ & COU812 & 2011.576 & 1 & 0.656 & 0.007 & $263.8^{*}$ & 0.3 & & & Cou1999b & -0.02 & -18.1 \\
\hline $18096+0400$ & STF2281 & 2011.527 & 1 & 0.655 & 0.007 & $287.2^{*}$ & 0.3 & & & Sod1999 & -0.01 & 1.4 \\
\hline $18101+1629$ & STF2289 & 2011.521 & 1 & 1.224 & 0.007 & $219.0^{*}$ & 0.4 & & & Hop1964b & -0.02 & 2.7 \\
\hline $18101+6007$ & MLR5 & 2012.531 & 1 & - & - & - & - & & NR & & & \\
\hline $18101+6007$ & MLR5 & 2011.516 & 1 & 0.359 & 0.077 & 42.1 & 8.0 & & & & & \\
\hline $18103+0235$ & BU638BC & 2011.510 & 1 & 1.810 & 0.018 & $187.8^{*}$ & 0.3 & & & & & \\
\hline $18103+0235$ & BU638BC & 2011.521 & 1 & 1.810 & 0.009 & $7.7^{*}$ & 0.3 & & & & & \\
\hline $18106+0738$ & HDS2562 & 2011.527 & 1 & 0.961 & 0.021 & 63.7 & 1.6 & & & & & \\
\hline $18109+3321$ & COU1005AB & 2012.575 & 1 & 0.182 & 0.040 & 143.7 & 8.0 & & & & & \\
\hline $18109+3321$ & COU1005AC & 2012.575 & 1 & 1.796 & 0.009 & 24.4 & 0.5 & & & & & \\
\hline $18113+6022$ & MLR501 & 2011.516 & 1 & 0.299 & 0.037 & 84.9 & 6.3 & & & & & \\
\hline $18113+6022$ & MLR501 & 2012.531 & 1 & 0.354 & 0.041 & 96.7 & 8.2 & & & & & \\
\hline $18114+2519$ & A238 & 2012.632 & 1 & 0.623 & 0.007 & $71.8^{*}$ & 0.4 & & & & & \\
\hline $18121+4644$ & COU2118 & 2011.573 & 1 & - & - & - & - & & NR & & & \\
\hline $18121+4644$ & COU2118 & 2012.589 & 1 & - & - & - & - & & NR & & & \\
\hline $18122+0814$ & HDS2568 & 2011.527 & 1 & 0.610 & 0.007 & $260.9^{*}$ & 0.6 & & & & & \\
\hline $18130+3318$ & COU1006 & 2012.479 & 1 & 0.455 & 0.007 & $326.6^{*}$ & 0.3 & & & Cou1999b & 0.25 & 99.7 \\
\hline $18131+2602$ & LWR14 & 2012.630 & 2 & - & - & - & - & & NR & & & \\
\hline $18132+5749$ & HDS2571 & 2011.518 & 1 & 0.257 & 0.008 & $300.0^{*}$ & 1.4 & & & & & \\
\hline $18133+0906$ & HDS2573 & 2011.527 & 1 & 0.821 & 0.007 & $163.3^{*}$ & 0.3 & & & & & \\
\hline
\end{tabular}




\begin{tabular}{|c|c|c|c|c|c|c|c|c|c|c|c|c|}
\hline WDS & Name & Epoch & Bin. & $\begin{array}{c}\rho \\
(")\end{array}$ & $\begin{array}{l}\sigma_{\rho} \\
(")\end{array}$ & $\begin{array}{c}\theta \\
\left({ }^{\circ}\right)\end{array}$ & $\begin{array}{l}\sigma_{\theta} \\
\left({ }^{\circ}\right)\end{array}$ & $\Delta m$ & Notes & Orbit & $\begin{array}{r}\Delta \rho(\mathrm{O}-\mathrm{C}) \\
(")\end{array}$ & $\begin{array}{r}\Delta \theta(\mathrm{O}-\mathrm{C}) \\
\left({ }^{\circ}\right)\end{array}$ \\
\hline $18133+2118$ & TDT743 & 2012.627 & 1 & 0.969 & 0.007 & $64.2^{*}$ & 0.8 & & & & & \\
\hline $18136+0946$ & HDS2574 & 2011.527 & 1 & 0.641 & 0.033 & 137.4 & 1.4 & & & & & \\
\hline $18136+0946$ & HDS2574 & 2011.527 & 1 & 0.673 & 0.014 & $136.5^{*}$ & ${ }^{*} 0.4$ & & & & & \\
\hline $18141+2235$ & TDT755 & 2012.605 & 1 & 1.756 & 0.009 & $77.1^{*}$ & 0.3 & & & & & \\
\hline $18144+1953$ & HDS2576 & 2011.573 & 1 & 0.973 & 0.017 & $67.1^{*}$ & 0.3 & & & & & \\
\hline $18144+1953$ & HDS2576 & 2012.548 & 1 & 0.957 & 0.017 & $66.3^{*}$ & 0.5 & & & & & \\
\hline $18144+1953$ & HDS2576 & 2011.573 & 1 & 0.947 & 0.017 & $65.5^{*}$ & 0.3 & & & & & \\
\hline $18144+4042$ & TDS910 & 2012.632 & 1 & 1.979 & 0.010 & $264.4^{*}$ & ${ }^{*} 0.3$ & & & & & \\
\hline $18145+3249$ & HU927 & 2012.520 & 1 & 0.354 & 0.007 & $101.7^{*}$ & ${ }^{*} 0.5$ & & & & & \\
\hline $18145+3313$ & COU1007 & 2012.570 & 1 & 0.290 & 0.025 & 37.5 & 0.8 & & & & & \\
\hline $18145+3313$ & COU1007 & 2012.570 & 1 & 0.279 & 0.035 & 38.6 & 3.5 & 0.81 & & & & \\
\hline $18146+0011$ & STF2294 & 2011.527 & 1 & 1.352 & 0.013 & $93.5^{*}$ & 0.3 & & & Sca2015b & 0.04 & 0.1 \\
\hline $18146+2335$ & HU318 & 2012.632 & 1 & 0.682 & 0.007 & $155.4^{*}$ & ${ }^{*} 0.5$ & & & & & \\
\hline $18147+5635$ & BU1274BC & 2011.516 & 2 & 1.080 & 0.015 & $145.5^{*}$ & ${ }^{*} 0.7$ & & & & & \\
\hline $18147+5635$ & BU1274BD & 2011.516 & 2 & 5.184 & 0.026 & $187.2^{*}$ & ${ }^{*} 0.3$ & & & & & \\
\hline $18153+6012$ & MLR6 & 2012.531 & 1 & 0.139 & 0.040 & 57.7 & 8.0 & & & & & \\
\hline $18153+6012$ & MLR6 & 2012.531 & 1 & 0.226 & 0.037 & 52.2 & 4.3 & & & & & \\
\hline $18154+5720$ & HDS2577 & 2011.516 & 1 & 0.235 & 0.013 & $292.4^{*}$ & ${ }^{*} 1.1$ & & & RAO2015 & -0.02 & -0.5 \\
\hline $18163+2333$ & TDT768 & 2012.605 & 1 & 0.139 & 0.040 & 126.2 & 8.0 & & & & & \\
\hline $18163+3625$ & HU1291 & 2011.573 & 1 & - & - & - & - & & NR & & & \\
\hline $18165+2249$ & HU319 & 2012.570 & 1 & 0.247 & 0.086 & 120.0 & 9.2 & 0.87 & & & & \\
\hline $18171+3453$ & COU12 & 2011.576 & 1 & 2.050 & 0.019 & $30.2^{*}$ & 0.3 & & & & & \\
\hline $18171+3453$ & COU12 & 2012.479 & 1 & 2.038 & 0.010 & $31.4^{*}$ & 0.5 & & & & & \\
\hline $18171+3453$ & COU12 & 2012.548 & 1 & 2.015 & 0.010 & $31.2^{*}$ & 0.3 & & & & & \\
\hline $18171+3453$ & COU12 & 2012.570 & 1 & 2.072 & 0.010 & 30.7 & 0.4 & 0.32 & & & & \\
\hline $18171+3453$ & COU12 & 2012.570 & 1 & 2.007 & 0.025 & 29.9 & 0.3 & & & & & \\
\hline $18177+3932$ & TDT778 & 2012.575 & 1 & 0.908 & 0.010 & $177.5^{*}$ & ${ }^{*} 0.8$ & & & & & \\
\hline $18178+4351$ & $\mathrm{~A} 578 \mathrm{AB}$ & 2012.520 & 1 & 1.845 & 0.009 & $167.3^{*}$ & ${ }^{*} 0.3$ & & & & & \\
\hline $18178+4351$ & $\mathrm{~A} 578 \mathrm{AB}$ & 2012.520 & 1 & 1.807 & 0.010 & $167.4^{*}$ & ${ }^{*} 0.3$ & & & & & \\
\hline $18178+4351$ & $\mathrm{~A} 578 \mathrm{AB}$ & 2012.586 & 1 & 1.796 & 0.019 & $168.2^{*}$ & ${ }^{*} 0.3$ & & & & & \\
\hline $18178+4351$ & $\mathrm{~A} 578 \mathrm{Aa}, \mathrm{Ab}$ & 2012.520 & 1 & 0.323 & 0.017 & $244.3^{*}$ & ${ }^{*} 0.3$ & & & & & \\
\hline $18178+4351$ & $\mathrm{~A} 578 \mathrm{Aa}, \mathrm{Ab}$ & 2012.520 & 1 & 0.298 & 0.017 & $243.0^{*}$ & ${ }^{*} 0.5$ & & & & & \\
\hline $18178+4351$ & $\mathrm{~A} 578 \mathrm{Aa}, \mathrm{Ab}$ & 2012.586 & 1 & 0.277 & 0.009 & $244.2^{*}$ & ${ }^{*} 1.2$ & & & & & \\
\hline $18181+6051$ & FOX81AB & 2011.516 & 1 & 0.887 & 0.007 & $132.3^{*}$ & 0.7 & & & & & \\
\hline $18182+4509$ & HDS2588 & 2012.589 & 1 & 0.181 & 0.040 & 27.7 & 8.0 & & & & & \\
\hline $18186+2513$ & TDT789 & 2012.583 & 1 & 1.025 & 0.007 & $82.3^{*}$ & 0.3 & & & & & \\
\hline $18187-1837$ & BU639AC & 2011.751 & 1 & 1.772 & 0.010 & $243.8^{*}$ & * 0.3 & & & & & \\
\hline $18198+1705$ & TDT799 & 2012.605 & 1 & 0.496 & 0.007 & $35.2^{*}$ & 0.3 & & & & & \\
\hline $18201-0759$ & STF2303 & 2012.548 & 1 & 0.761 & 0.007 & $293.3^{*}$ & ${ }^{*} 0.3$ & & & & & \\
\hline $18203+2104$ & HDS2592 & 2011.559 & 1 & 0.449 & 0.042 & $183.6^{*}$ & ${ }^{*} 1.6$ & & & & & \\
\hline $18204+2423$ & TDT805 & 2012.575 & 1 & 0.979 & 0.011 & 57.8 & 1.1 & 0.22 & & & & \\
\hline $18204+2423$ & TDT805 & 2012.575 & 1 & 0.978 & 0.007 & $56.8^{*}$ & 0.3 & & & & & \\
\hline $18204+2423$ & TDT805 & 2012.575 & 1 & 0.999 & 0.030 & 55.7 & 1.4 & & & & & \\
\hline $18205+2055$ & COU202 & 2012.575 & 1 & 0.156 & 0.040 & 113.4 & 8.0 & & & & & \\
\hline $18207+3547$ & COU1148 & 2012.586 & 1 & 1.873 & 0.014 & $71.5^{*}$ & 0.7 & & & & & \\
\hline $18213+2409$ & COU505 & 2011.557 & 1 & 1.041 & 0.011 & $138.0^{*}$ & ${ }^{*} 0.3$ & & & & & \\
\hline $18214+1810$ & HDS2594 & 2012.605 & 1 & 0.259 & 0.007 & $319.2^{*}$ & ${ }^{*} 0.4$ & & & & & \\
\hline $18215+1318$ & HDS2595 & 2011.527 & 1 & 1.551 & 0.008 & $23.6^{*}$ & 0.3 & & & & & \\
\hline $18217+0902$ & HDS2596 & 2011.527 & 1 & - & - & - & - & & NR & & & \\
\hline $18217+2356$ & COU418 & 2012.518 & 1 & 0.192 & 0.040 & $277.3^{*}$ & ${ }^{*} 8.0$ & & & & & \\
\hline $18217+2356$ & COU418 & 2011.557 & 1 & 0.216 & 0.024 & $265.3^{*}$ & ${ }^{*} 2.8$ & & & & & \\
\hline $18222+3417$ & TDT818 & 2012.635 & 1 & 0.282 & 0.007 & $202.4^{*}$ & ${ }^{*} 0.9$ & & & & & \\
\hline $18224+4545$ & A700 & 2012.635 & 1 & 0.625 & 0.007 & $127.9^{*}$ & ${ }^{*} 0.5$ & & & Hei1998 & 0.49 & -19.8 \\
\hline $18225+7659$ & HU930 & 2012.531 & 1 & 1.024 & 0.007 & $309.5^{*}$ & ${ }^{*} 0.3$ & & & & & \\
\hline
\end{tabular}




\begin{tabular}{|c|c|c|c|c|c|c|c|c|c|c|c|c|}
\hline WDS & Name & Epoch & Bin. & $\begin{array}{c}\rho \\
(")\end{array}$ & $\begin{array}{l}\sigma_{\rho} \\
(")\end{array}$ & $\begin{array}{c}\theta \\
\left({ }^{\circ}\right)\end{array}$ & $\begin{array}{l}\sigma_{\theta} \\
\left(^{\circ}\right)\end{array}$ & $\Delta m$ & Notes & Orbit & $\begin{array}{r}\Delta \rho(\mathrm{O}-\mathrm{C}) \\
(")\end{array}$ & $\begin{array}{r}\Delta \theta(\mathrm{O}-\mathrm{C}) \\
\left({ }^{\circ}\right)\end{array}$ \\
\hline $18225+7659$ & HU930 & 2012.531 & 1 & 1.034 & 0.032 & 311.4 & 2.6 & & & & & \\
\hline $18238+5318$ & YSC66 & 2012.531 & 1 & 0.886 & 0.007 & $42.7^{*}$ & 0.3 & & & & & \\
\hline $18238+5318$ & YSC66 & 2012.531 & 1 & 0.872 & 0.013 & 43.4 & 0.4 & & & & & \\
\hline $18238+5318$ & YSC66 & 2012.531 & 1 & - & - & - & - & & NR & & & \\
\hline $18241+4054$ & COU1603 & 2012.632 & 1 & 0.280 & 0.009 & $82.6^{*}$ & 0.9 & & & & & \\
\hline $18242+2818$ & A244 & 2011.557 & 1 & 0.548 & 0.011 & $272.3^{*}$ & 1.5 & & & & & \\
\hline $18249+1932$ & TDT845 & 2012.605 & 1 & - & - & - & - & & NR & & & \\
\hline $18250-0135$ & $\mathrm{AC} 11$ & 2011.521 & 1 & 0.914 & 0.007 & $355.6^{*}$ & 0.3 & & & Tok2017c & 0.01 & 0.6 \\
\hline $18250+2724$ & STF 2315 & 2011.557 & 1 & 0.638 & 0.007 & $120.0^{*}$ & 0.4 & & & WSI2004b & 0.02 & 1.5 \\
\hline $18250+2724$ & STF2315 & 2012.520 & 1 & 0.636 & 0.007 & $118.8^{*}$ & 0.3 & & & WSI2004b & 0.02 & 0.7 \\
\hline $18253+2605$ & HDS2605 & 2012.632 & 1 & 1.203 & 0.007 & $83.3^{*}$ & 0.3 & & & & & \\
\hline $18253+4846$ & HU66 & 2011.559 & 1 & 0.211 & 0.007 & 213.8 & 2.3 & 0.50 & & USN2002 & 0.05 & 0.9 \\
\hline $18253+4846$ & HU66 & 2011.573 & 1 & 0.191 & 0.040 & $216.9^{*}$ & 8.0 & 0.46 & & USN2002 & 0.03 & 4.0 \\
\hline $18253+4846$ & STT351AC & 2011.559 & 1 & 0.786 & 0.028 & $26.0^{*}$ & 0.3 & & & & & \\
\hline $18253+4846$ & STT351AC & 2011.573 & 1 & 0.766 & 0.027 & $25.9^{*}$ & 0.5 & & & & & \\
\hline $18257+2639$ & COU815 & 2011.557 & 2 & 1.596 & 0.024 & $146.6^{*}$ & 0.8 & & & & & \\
\hline $18264+0255$ & HEI560 & 2011.521 & 1 & 1.705 & 0.009 & $208.7^{*}$ & 0.3 & & & & & \\
\hline $18266+0633$ & HDS2607 & 2011.521 & 1 & 0.173 & 0.040 & 17.6 & 8.0 & & & & & \\
\hline $18271+2754$ & TDS921 & 2012.586 & 1 & 0.911 & 0.007 & $135.0^{*}$ & 0.5 & & & & & \\
\hline $18276+4926$ & COU2278 & 2011.559 & 1 & 1.409 & 0.007 & $15.8^{*}$ & 0.3 & & & & & \\
\hline $18285+2010$ & COU203AB & 2012.518 & 1 & 0.177 & 0.040 & 154.4 & 8.0 & & & & & \\
\hline $18285+2010$ & COU203AC & 2012.518 & 1 & 0.465 & 0.007 & $56.4^{*}$ & 0.3 & & & & & \\
\hline $18295+3259$ & TDS925 & 2012.586 & 1 & 1.756 & 0.009 & $89.3^{*}$ & 0.3 & & & & & \\
\hline $18295+3259$ & TDS925 & 2012.589 & 1 & 1.791 & 0.009 & $88.6^{*}$ & 0.3 & & & & & \\
\hline $18295+3259$ & TDS925 & 2012.627 & 1 & 1.773 & 0.018 & $88.2^{*}$ & 0.3 & & & & & \\
\hline $18296+2603$ & HDS2619 & 2012.518 & 1 & 0.620 & 0.007 & 109.8 & 0.3 & 4.53 & & & & \\
\hline $18297+3929$ & TDT910 & 2012.635 & 1 & 0.807 & 0.007 & $194.8^{*}$ & 0.3 & & & & & \\
\hline $18301+4325$ & J763 & 2011.573 & 1 & 1.838 & 0.010 & $309.8^{*}$ & 0.3 & & & & & \\
\hline $18305+0416$ & A583 & 2011.510 & 1 & - & - & - & - & & NR & & & \\
\hline $18306+4429$ & COU1928 & 2012.518 & 1 & 0.559 & 0.007 & $272.8^{*}$ & 0.3 & & & & & \\
\hline $18309+3417$ & COU1150 & 2012.548 & 1 & 0.176 & 0.040 & 100.8 & 8.0 & & & & & \\
\hline $18311+2216$ & COU15 & 2011.573 & 1 & 1.948 & 0.010 & $118.4^{*}$ & 0.3 & & & & & \\
\hline $18316+2030$ & COU119 & 2011.573 & 1 & 0.675 & 0.008 & $227.5^{*}$ & 0.3 & & & & & \\
\hline $18316+2030$ & COU119 & 2012.518 & 1 & 0.686 & 0.007 & $227.8^{*}$ & 0.3 & & & & & \\
\hline $18316+2030$ & COU119 & 2012.548 & 1 & 0.688 & 0.007 & $227.0^{*}$ & 1.0 & & & & & \\
\hline $18316+2030$ & COU119 & 2012.605 & 1 & 0.675 & 0.007 & $227.5^{*}$ & 0.3 & & & & & \\
\hline $18317+7120$ & A1170 & 2011.516 & 1 & 0.877 & 0.007 & $188.3^{*}$ & 0.3 & & & & & \\
\hline $18319+3538$ & COU1151 & 2012.556 & 1 & 1.289 & 0.015 & $96.5^{*}$ & 0.5 & & & & & \\
\hline $18323+1625$ & HDS2630 & 2011.510 & 1 & 0.158 & 0.040 & 165.0 & 8.0 & & & & & \\
\hline $18334+2301$ & $\mathrm{~J} 463$ & 2012.635 & 1 & 1.900 & 0.009 & $223.6^{*}$ & 0.3 & & & & & \\
\hline $18335+3510$ & $\mathrm{HO} 86$ & 2012.518 & 1 & 0.286 & 0.010 & $198.6^{*}$ & 0.5 & & & & & \\
\hline $18335+3934$ & COU1606 & 2012.627 & 1 & 1.895 & 0.009 & $130.2^{*}$ & 0.3 & & & & & \\
\hline $18335+3934$ & COU1606 & 2012.627 & 1 & 1.886 & 0.009 & $130.1^{*}$ & 0.3 & & & & & \\
\hline $18335+6929$ & MLR95 & 2011.516 & 1 & 0.224 & 0.021 & 43.7 & 3.3 & & & & & \\
\hline $18337+6019$ & FOX83 & 2011.516 & 1 & 0.686 & 0.007 & $27.9^{*}$ & 0.8 & & & & & \\
\hline $18338+1744$ & WAK21AB & 2012.556 & 1 & 1.553 & 0.018 & 96.4 & 0.6 & 0.63 & & & & \\
\hline $18338+1744$ & WAK21AB & 2012.605 & 1 & 1.570 & 0.018 & 96.4 & 0.6 & & & & & \\
\hline $18338+1744$ & WAK21CD & 2012.583 & 1 & 0.474 & 0.007 & 261.5 & 0.3 & 0.68 & & & & \\
\hline $18338+1744$ & WAK21CD & 2012.605 & 1 & 0.494 & 0.007 & 262.6 & 0.3 & 0.67 & & & & \\
\hline $18338+1744$ & STF2339AB,CD & 2012.583 & 1 & 1.556 & 0.008 & $276.3^{*}$ & 0.3 & & & & & \\
\hline $18338+1744$ & STF2339AB,CD & 2012.605 & 1 & 1.564 & 0.008 & 276.8 & 0.3 & & & & & \\
\hline $18339+7016$ & MLR97 & 2011.516 & 1 & 0.232 & 0.012 & 110.5 & 5.0 & & & & & \\
\hline $18347+3548$ & HDS2635AC & 2011.559 & 1 & 0.255 & 0.015 & 95.1 & 2.6 & & & & & \\
\hline $18354-0621$ & RST4591 & 2011.527 & 1 & 0.266 & 0.007 & 11.6 & 0.3 & & & & & \\
\hline
\end{tabular}




\begin{tabular}{|c|c|c|c|c|c|c|c|c|c|c|c|c|}
\hline WDS & Name & Epoch & Bin. & $\begin{array}{c}\rho \\
(")\end{array}$ & $\begin{array}{l}\sigma_{\rho} \\
(")\end{array}$ & $\begin{array}{c}\theta \\
\left({ }^{\circ}\right)\end{array}$ & $\begin{array}{l}\sigma_{\theta} \\
\left(^{\circ}\right)\end{array}$ & $\Delta m$ & Notes & Orbit & $\begin{array}{r}\Delta \rho(\mathrm{O}-\mathrm{C}) \\
(")\end{array}$ & $\begin{array}{r}\Delta \theta(\mathrm{O}-\mathrm{C}) \\
\left({ }^{\circ}\right)\end{array}$ \\
\hline $18355+2336$ & STT359 & 2011.559 & 1 & 0.741 & 0.007 & $6.8^{*}$ & 0.4 & & & Sca2009a & 0.00 & 2.0 \\
\hline $18359+1659$ & STT358 & 2012.556 & 1 & 1.619 & 0.022 & 150.2 & 0.5 & & & Izm2019 & -0.03 & 0.6 \\
\hline $18359+2542$ & TDT952 & 2012.630 & 1 & 1.109 & 0.007 & $69.0^{*}$ & 0.3 & & & & & \\
\hline $18360+3452$ & GII48 & 2012.630 & 1 & 0.345 & 0.007 & $315.3^{*}$ & 0.3 & & $\mathrm{ND}$ & & & \\
\hline $18360+3452$ & GII48 & 2012.632 & 1 & 0.359 & 0.010 & $316.1^{*}$ & 0.6 & & $\mathrm{ND}$ & & & \\
\hline $18361+2003$ & TDS931 & 2012.586 & 1 & 1.252 & 0.007 & $48.6^{*}$ & 0.5 & & & & & \\
\hline $18361+2344$ & HDS2639 & 2011.573 & 1 & 0.108 & 0.040 & 135.9 & 5.7 & & & & & \\
\hline $18361+2344$ & HDS2639 & 2012.583 & 1 & 0.076 & 0.040 & 137.0 & 5.5 & & & & & \\
\hline $18363+2143$ & COU206 & 2011.559 & 1 & - & - & - & - & & NR & & & \\
\hline $18363+2143$ & COU206 & 2012.518 & 1 & - & - & - & - & & NR & & & \\
\hline $18366+3505$ & COU1307 & 2011.559 & 1 & 1.134 & 0.013 & $326.6^{*}$ & 0.3 & & & & & \\
\hline $18377+3346$ & TDT963 & 2012.586 & 1 & 1.190 & 0.007 & $149.7^{*}$ & 0.3 & & & & & \\
\hline $18384-0312$ & $\mathrm{~A} 88 \mathrm{AB}$ & 2011.527 & 1 & - & - & - & - & & NR & & & \\
\hline $18384+0850$ & HU198 & 2011.510 & 1 & 0.522 & 0.007 & $129.5^{*}$ & 0.5 & & & Nov2007d & 0.06 & 0.1 \\
\hline $18385+3503$ & COU1308 & 2012.518 & 1 & 0.369 & 0.007 & 219.4 & 0.3 & 1.90 & & & & \\
\hline $18385+3503$ & COU1308 & 2012.518 & 1 & 0.320 & 0.007 & 210.8 & 1.1 & & & & & \\
\hline $18386+1632$ & $\mathrm{HO} 87$ & 2012.635 & 1 & 0.440 & 0.007 & $87.4^{*}$ & 0.5 & & & Msn2019 & 0.02 & -1.0 \\
\hline $18389+2324$ & TDT972 & 2012.635 & 1 & 0.848 & 0.007 & $43.8^{*}$ & 0.4 & & & & & \\
\hline $18390+5559$ & A1380AB & 2011.516 & 1 & 0.998 & 0.007 & $200.3^{*}$ & 0.3 & & & & & \\
\hline $18399+3806$ & HU1190 & 2011.573 & 1 & 1.638 & 0.008 & $168.3^{*}$ & 0.3 & & & & & \\
\hline $18402+3822$ & HDS2644 & 2011.559 & 1 & - & - & - & - & & NR & & & \\
\hline $18403+3425$ & HDS2645 & 2011.559 & 1 & 1.908 & 0.010 & $20.4^{*}$ & 0.3 & & & & & \\
\hline $18403+3425$ & HDS2645 & 2012.586 & 1 & 1.917 & 0.010 & 19.1 & 0.3 & 4.74 & & & & \\
\hline $18403+3425$ & HDS2645 & 2012.586 & 1 & 1.881 & 0.009 & $19.6^{*}$ & 0.3 & & & & & \\
\hline $18403+3425$ & HDS2645 & 2012.627 & 1 & 1.889 & 0.010 & 18.8 & 0.3 & & & & & \\
\hline $18403+3425$ & HDS2645 & 2012.627 & 1 & 1.889 & 0.009 & $19.0^{*}$ & 0.3 & & & & & \\
\hline $18407+4336$ & TDT990 & 2012.575 & 1 & 0.340 & 0.007 & 138.6 & 1.2 & & & & & \\
\hline $18407+4336$ & TDT990 & 2012.575 & 1 & 0.296 & 0.027 & 134.8 & 2.5 & & & & & \\
\hline $18410+2450$ & A2988 & 2012.556 & 1 & 0.135 & 0.040 & 220.4 & 8.0 & & & FMR2010f & 0.01 & -8.6 \\
\hline $18413+3018$ & STF2367 & 2011.576 & 1 & 0.415 & 0.008 & $74.6^{*}$ & 0.3 & & & Pbx2000b & 0.01 & 0.5 \\
\hline $18416-0353$ & RST4593 & 2011.527 & 1 & - & - & - & - & & NR & & & \\
\hline $18421+3445$ & $\mathrm{~B} 2546 \mathrm{Aa}, \mathrm{Ab}$ & 2012.556 & 1 & 0.151 & 0.040 & 8.7 & 8.0 & & & USN2002 & 0.04 & $-4.1^{Q}$ \\
\hline $18427+0742$ & HEI870 & 2011.521 & 1 & 1.542 & 0.056 & $345.3^{*}$ & 5.9 & & & & & \\
\hline $18431+2447$ & TDS937 & 2012.632 & 1 & 1.675 & 0.025 & $44.0^{*}$ & 0.8 & & & & & \\
\hline $18432+3822$ & HDS2651 & 2011.573 & 1 & 0.476 & 0.007 & $46.1^{*}$ & 0.7 & & & & & \\
\hline $18433+1847$ & COU816 & 2012.583 & 1 & 0.230 & 0.007 & $121.9^{*}$ & 1.1 & & & & & \\
\hline $18440+0321$ & A2388 & 2011.510 & 1 & 0.277 & 0.007 & $127.9^{*}$ & 0.9 & & & FMR2014c & 0.02 & -1.7 \\
\hline $18443+2720$ & TDS941 & 2012.630 & 1 & 0.835 & 0.007 & $91.5^{*}$ & 0.3 & & & & & \\
\hline $18449+0253$ & HEI562 & 2011.510 & 1 & 0.345 & 0.008 & $246.9^{*}$ & 0.4 & & & & & \\
\hline $18450+4051$ & COU1792 & 2011.557 & 2 & 1.075 & 0.015 & $347.2^{*}$ & 0.5 & & & & & \\
\hline $18451+0639$ & HDS2658 & 2011.527 & 1 & 0.110 & 0.063 & 200.7 & 8.6 & & & & & \\
\hline $18451+0639$ & HDS2658 & 2011.527 & 1 & 0.125 & 0.051 & 190.3 & 8.9 & & & & & \\
\hline $18455+0530$ & FIN332 & 2011.521 & 1 & 2.562 & 0.013 & $120.2^{*}$ & 0.3 & & & & & \\
\hline $18459+1450$ & HEI397 & 2011.576 & 1 & 1.087 & 0.007 & $141.1^{*}$ & 0.3 & & & & & \\
\hline $18459+3657$ & COU1309 & 2012.575 & 1 & 0.484 & 0.027 & 179.5 & 3.6 & & & & & \\
\hline $18459+3657$ & COU1309 & 2012.575 & 1 & 0.471 & 0.019 & 180.3 & 0.7 & 0.05 & & & & \\
\hline $18461+1655$ & HEI259 & 2012.682 & 1 & 1.921 & 0.010 & $247.2^{*}$ & 0.3 & & & & & \\
\hline $18462+4408$ & HDS2662 & 2012.548 & 1 & 0.770 & 0.007 & $256.6^{*}$ & 0.3 & & & & & \\
\hline $18465+3414$ & COU1153 & 2012.586 & 1 & 0.364 & 0.010 & $115.6^{*}$ & 0.3 & & & & & \\
\hline $18465+3414$ & COU1153 & 2012.605 & 1 & 0.367 & 0.007 & $115.1^{*}$ & 0.5 & & & & & \\
\hline $18466+3821$ & HU1191 & 2011.573 & 1 & 0.138 & 0.040 & 121.4 & 8.0 & & & Doc2009g & 0.04 & 7.0 \\
\hline $18476+3248$ & COU1154 & 2012.605 & 1 & 0.566 & 0.008 & $54.3^{*}$ & 0.7 & & & & & \\
\hline $18476+4450$ & KOI68AB & 2012.627 & 1 & - & - & - & - & & NR & & & \\
\hline
\end{tabular}




\begin{tabular}{|c|c|c|c|c|c|c|c|c|c|c|c|c|}
\hline WDS & Name & Epoch & Bin. & $\begin{array}{c}\rho \\
(")\end{array}$ & $\begin{array}{l}\sigma_{\rho} \\
(")\end{array}$ & $\begin{array}{c}\theta \\
\left({ }^{\circ}\right)\end{array}$ & $\begin{array}{l}\sigma_{\theta} \\
\left({ }^{\circ}\right)\end{array}$ & $\Delta m$ & Notes & Orbit & $\begin{array}{r}\Delta \rho(\mathrm{O}-\mathrm{C}) \\
(")\end{array}$ & $\begin{array}{r}\Delta \theta(\mathrm{O}-\mathrm{C}) \\
\left({ }^{\circ}\right)\end{array}$ \\
\hline $18476+4450$ & KOI68AB & 2012.635 & 1 & - & - & 一 & - & & $\mathrm{NR}$ & & & \\
\hline $18486+3132$ & TDT1063 & 2012.605 & 1 & 0.262 & 0.007 & 109.8 & 1.2 & & & & & \\
\hline $18486+3132$ & TDT1063 & 2012.575 & 1 & 0.325 & 0.007 & 112.1 & 1.7 & & & & & \\
\hline $18489+1615$ & STF2400BC & 2011.527 & 1 & 0.975 & 0.007 & $201.8^{*}$ & 0.7 & & & & & \\
\hline $18489+1615$ & STF2400BC & 2011.510 & 1 & 0.989 & 0.007 & $22.8^{*}$ & 0.3 & & & & & \\
\hline $18490-0828$ & HDS2666 & 2011.521 & 1 & 0.220 & 0.009 & $261.6^{*}$ & 0.9 & & & & & \\
\hline $18490+3432$ & YSC11 & 2012.556 & 1 & 0.266 & 0.079 & 74.1 & 7.8 & & & & & \\
\hline $18495+1811$ & HDS2668 & 2011.527 & 1 & 0.393 & 0.007 & $60.3^{*}$ & 0.3 & & & & & \\
\hline $18497+6930$ & MLR96 & 2011.516 & 1 & 1.649 & 0.008 & 86.4 & 0.3 & & & & & \\
\hline $18500+1519$ & YSC12AB & 2012.556 & 1 & 0.443 & 0.024 & 55.2 & 2.5 & & & Tok2018b & -0.04 & -0.1 \\
\hline $18500+1519$ & YSC12AB & 2012.632 & 1 & - & - & - & - & & NR & & & \\
\hline $18500+1519$ & YSC12AB & 2012.635 & 1 & 0.438 & 0.015 & 53.2 & 2.4 & & & Tok2018b & -0.04 & -1.9 \\
\hline $18509+2058$ & COU317 & 2012.682 & 1 & 1.521 & 0.008 & $94.6^{*}$ & 0.3 & & & & & \\
\hline $18511-0540$ & RST4603 & 2011.521 & 1 & 0.747 & 0.007 & $100.4^{*}$ & 0.4 & & & & & \\
\hline $18511-0540$ & RST4603 & 2011.521 & 2 & 0.739 & 0.015 & $98.6^{*}$ & 0.8 & & & & & \\
\hline $18517+4323$ & BU421 & 2012.641 & 1 & 1.171 & 0.007 & $296.4^{*}$ & 0.3 & & & & & \\
\hline $18521+2431$ & COU510 & 2012.589 & 1 & 0.180 & 0.040 & $165.6^{*}$ & 8.0 & & & & & \\
\hline $18525+2632$ & HDS2677 & 2012.589 & 1 & 0.985 & 0.007 & 76.0 & 0.3 & & & & & \\
\hline $18526+4508$ & KOI42 & 2012.589 & 1 & 1.671 & 0.008 & $34.9^{*}$ & 0.3 & & & & & \\
\hline $18528+3125$ & $\mathrm{~A} 257 \mathrm{CD}$ & 2011.559 & 1 & 0.682 & 0.030 & $82.8^{*}$ & 1.4 & & & & & \\
\hline $18528+3125$ & $\mathrm{~A} 257 \mathrm{CD}$ & 2012.589 & 1 & 0.734 & 0.007 & $81.6^{*}$ & 0.3 & & & & & \\
\hline $18529+4121$ & KOI284 & 2012.586 & 1 & 0.831 & 0.007 & $95.5^{*}$ & 0.4 & & & & & \\
\hline $18529+4121$ & KOI284 & 2012.589 & 1 & 0.858 & 0.007 & $96.2^{*}$ & 0.4 & & & & & \\
\hline $18529+4121$ & KOI284 & 2012.627 & 1 & 0.840 & 0.007 & $94.8^{*}$ & 0.3 & & & & & \\
\hline $18529+5814$ & MLR359 & 2011.516 & 1 & 1.838 & 0.009 & $294.2^{*}$ & 0.3 & & & & & \\
\hline $18534+2553$ & A2989 & 2012.556 & 1 & 0.297 & 0.023 & 197.0 & 3.1 & & & USN2002 & 0.03 & -2.0 \\
\hline $18550+3053$ & A 258 & 2012.693 & 1 & 0.323 & 0.007 & $237.7^{*}$ & 1.0 & & & Hrt2013c & -0.05 & -3.0 \\
\hline $18551+4051$ & HEI73 & 2012.630 & 1 & 0.706 & 0.007 & $320.8^{*}$ & 0.6 & & & & & \\
\hline $18552+3941$ & TDT1126 & 2012.687 & 1 & 0.340 & 0.007 & $189.6^{*}$ & 0.4 & & & & & \\
\hline $18554+2324$ & COU511 & 2012.556 & 1 & 0.665 & 0.208 & 185.0 & 21.0 & & & & & \\
\hline $18554+3556$ & A1385 & 2012.575 & 1 & 0.477 & 0.007 & 112.9 & 0.5 & & & & & \\
\hline $18554+3556$ & A1385 & 2012.575 & 1 & 0.499 & 0.180 & 119.9 & 12.9 & 1.29 & & & & \\
\hline $18563-0048$ & A1171AB & 2011.527 & 1 & 0.873 & 0.007 & $81.0^{*}$ & 0.3 & & & & & \\
\hline $18564+2944$ & COU1014 & 2012.630 & 1 & 0.240 & 0.007 & $43.5^{*}$ & 0.9 & & & & & \\
\hline $18571+3451$ & HDS2685 & 2012.641 & 1 & 0.506 & 0.007 & $199.3^{*}$ & 0.6 & & & & & \\
\hline $18572+3845$ & COU1611 & 2012.687 & 1 & 0.666 & 0.007 & $108.0^{*}$ & 0.5 & & & & & \\
\hline $18574+2129$ & HU329 & 2012.693 & 1 & 0.107 & 0.040 & 157.9 & 8.0 & & & & & \\
\hline $18582+4858$ & KOI258AB & 2012.586 & 1 & 1.032 & 0.030 & 72.9 & 0.3 & & & & & \\
\hline $18582+4858$ & KOI258AB & 2012.586 & 1 & 1.002 & 0.030 & $72.9^{*}$ & 0.3 & & & & & \\
\hline $18582+4858$ & KOI258AB & 2012.589 & 1 & 1.010 & 0.030 & $73.5^{*}$ & 0.4 & & & & & \\
\hline $18582+4858$ & KOI258AB & 2012.641 & 2 & 1.021 & 0.030 & 73.3 & 0.3 & 3.37 & & & & \\
\hline $18582+4858$ & KOI258AB & 2012.641 & 2 & 1.071 & 0.030 & 73.8 & 0.3 & & & & & \\
\hline $18582+4858$ & KOI258AB & 2012.641 & 1 & 0.976 & 0.030 & 72.1 & 0.3 & 1.33 & & & & \\
\hline $18582+4858$ & $\mathrm{KOI} 258 \mathrm{Aa}, \mathrm{Ab}$ & 2012.586 & 1 & 0.460 & 0.027 & 78.9 & 0.3 & 1.08 & & & & \\
\hline $18582+4858$ & $\mathrm{KOI} 258 \mathrm{Aa}, \mathrm{Ab}$ & 2012.589 & 1 & 0.436 & 0.027 & $79.0^{*}$ & 0.3 & 0.84 & & & & \\
\hline $18582+4858$ & $\mathrm{KOI} 258 \mathrm{Aa}, \mathrm{Ab}$ & 2012.641 & 1 & 0.436 & 0.027 & 80.3 & 0.3 & 0.85 & & & & \\
\hline $18582+7519$ & WOR26 & 2011.516 & 1 & 0.231 & 0.007 & 161.5 & 6.8 & & & & & \\
\hline $18587+2744$ & COU817 & 2011.557 & 2 & 1.066 & 0.016 & $194.4^{*}$ & 2.0 & & & & & \\
\hline $18589+2500$ & TDT1162 & 2012.605 & 1 & 0.986 & 0.027 & $176.7^{*}$ & 0.3 & & & & & \\
\hline $18589+2500$ & TDT1162 & 2012.687 & 1 & 1.004 & 0.027 & $177.6^{*}$ & 0.5 & & & & & \\
\hline $18597+2949$ & COU1019 & 2012.630 & 1 & 0.302 & 0.007 & $348.8^{*}$ & 0.6 & & & & & \\
\hline $19005+2716$ & TDT1178 & 2012.627 & 1 & 0.849 & 0.007 & $334.8^{*}$ & 0.3 & & & & & \\
\hline $19006+3300$ & COU1156 & 2011.559 & 1 & 0.780 & 0.007 & $290.4^{*}$ & 0.4 & & & & & \\
\hline
\end{tabular}




\begin{tabular}{|c|c|c|c|c|c|c|c|c|c|c|c|c|}
\hline WDS & Name & Epoch & Bin. & $\begin{array}{c}\rho \\
(")\end{array}$ & $\begin{array}{l}\sigma_{\rho} \\
(")\end{array}$ & $\begin{array}{c}\theta \\
\left({ }^{\circ}\right)\end{array}$ & $\begin{array}{l}\sigma_{\theta} \\
\left(^{\circ}\right)\end{array}$ & $\Delta m$ & Notes & Orbit & $\begin{array}{r}\Delta \rho(\mathrm{O}-\mathrm{C}) \\
(")\end{array}$ & $\begin{array}{r}\Delta \theta(\mathrm{O}-\mathrm{C}) \\
\left({ }^{\circ}\right)\end{array}$ \\
\hline $19006+3951$ & COU1933 & 2012.632 & 1 & 0.541 & 0.007 & $199.2^{*}$ & 0.4 & & & & & \\
\hline $19006+3952$ & HDS2696 & 2012.630 & 1 & 0.362 & 0.007 & 136.6 & 0.3 & & & & & \\
\hline $19016+3253$ & HU1295 & 2012.583 & 1 & 0.252 & 0.007 & $219.2^{*}$ & 1.2 & & & & & \\
\hline $19018+3448$ & COU1612 & 2012.682 & 1 & 0.854 & 0.007 & $321.5^{*}$ & 0.3 & & & & & \\
\hline $19027-0043$ & STF2434BC & 2011.527 & 1 & 0.691 & 0.010 & $273.3^{*}$ & 0.5 & & & & & \\
\hline $19027+4307$ & YSC13 & 2012.583 & 1 & 0.097 & 0.040 & 167.0 & 8.0 & & & Doc $2013 \mathrm{j}$ & 0.01 & -7.8 \\
\hline $19028+2208$ & TDS961 & 2012.684 & 1 & 0.888 & 0.007 & $211.6^{*}$ & 0.3 & & & & & \\
\hline $19029+2742$ & WAK3AC & 2012.682 & 1 & 1.145 & 0.028 & 356.9 & 0.3 & 1.90 & & & & \\
\hline $19029+2742$ & WAK3AC & 2012.682 & 1 & 1.126 & 0.025 & 357.2 & 0.3 & & & & & \\
\hline $19029+2742$ & WAK3AC & 2012.682 & 1 & 1.120 & 0.027 & 357.3 & 0.3 & 1.51 & & & & \\
\hline $19036+3705$ & HDS2702 & 2011.559 & 1 & 0.272 & 0.007 & 92.9 & 1.0 & & & & & \\
\hline $19038+4000$ & TDT1203 & 2012.687 & 1 & 1.177 & 0.007 & $203.9^{*}$ & 0.3 & & & & & \\
\hline $19038+4040$ & COU2196 & 2012.709 & 1 & 0.497 & 0.007 & $332.8^{*}$ & 0.3 & & & & & \\
\hline $19039+2642$ & A2992 & 2011.573 & 1 & 0.170 & 0.040 & 234.0 & 8.0 & & & Doc2009g & -0.03 & -1.4 \\
\hline $19039+2642$ & A2992 & 2012.632 & 1 & 0.193 & 0.040 & $235.3^{*}$ & 8.0 & & & Doc $2009 \mathrm{~g}$ & -0.00 & $1.3^{Q}$ \\
\hline $19045-0426$ & A2689 & 2011.521 & 1 & 0.744 & 0.007 & $69.2^{*}$ & 0.3 & & & & & \\
\hline $19045-0426$ & A2689 & 2011.521 & 1 & 0.739 & 0.007 & $70.0^{*}$ & 0.3 & & & & & \\
\hline $19053+1504$ & HEI267 & 2011.510 & 1 & 0.881 & 0.007 & $43.8^{*}$ & 0.4 & & & & & \\
\hline $19055+3352$ & HU940 & 2011.576 & 1 & 0.487 & 0.007 & $194.5^{*}$ & 0.5 & & & Doc $2009 \mathrm{~g}$ & -0.01 & 2.4 \\
\hline $19056+2724$ & HDS2709 & 2011.559 & 1 & 0.336 & 0.007 & $38.1^{*}$ & 1.0 & & & & & \\
\hline $19057+2717$ & HO95 & 2012.684 & 1 & 0.199 & 0.040 & $147.3^{*}$ & 8.0 & & & & & \\
\hline $19060+3354$ & TDS965 & 2012.693 & 1 & 1.370 & 0.007 & $166.1^{*}$ & 0.4 & & & & & \\
\hline $19069+4137$ & COU2197 & 2011.559 & 1 & 0.136 & 0.047 & $149.3^{*}$ & 6.8 & & & Doc $2016 \mathrm{~g}$ & -0.05 & $14.1^{Q}$ \\
\hline $19069+4137$ & COU2197 & 2012.627 & 1 & 0.148 & 0.044 & $146.8^{*}$ & 6.9 & & & Doc2016g & -0.03 & $20.3^{Q}$ \\
\hline $19070+1104$ & HEI568 & 2011.510 & 1 & 0.267 & 0.014 & $263.5^{*}$ & 1.5 & & & Zir2010 & -0.04 & -2.2 \\
\hline $19073+3606$ & STA3 & 2011.576 & 1 & - & - & - & - & & NR & & & \\
\hline $19074+3230$ & KUI90Ca,Cb & 2011.559 & 1 & 0.370 & 0.023 & $87.9^{*}$ & 1.5 & & & Sgr2000 & 0.03 & 8.2 \\
\hline $19074+3230$ & $\mathrm{KUI} 90 \mathrm{Ca}, \mathrm{Cb}$ & 2011.573 & 1 & 0.345 & 0.007 & $80.5^{*}$ & 0.4 & & & Sgr2000 & 0.03 & 8.2 \\
\hline $19074+3230$ & KUI90Ca,Cb & 2012.583 & 1 & 0.337 & 0.007 & $90.7^{*}$ & 0.8 & & & Sgr 2000 & 0.03 & 8.2 \\
\hline $19076-0249$ & HDS2711AB & 2011.521 & 1 & - & - & - & - & & NR & & & \\
\hline $19081+2142$ & CHR83 & 2012.632 & 1 & 0.128 & 0.040 & 161.6 & 8.0 & & & & & \\
\hline $19081+2142$ & CHR83 & 2011.576 & 1 & - & - & - & - & & NR & & & \\
\hline $19081+3031$ & HO99 & 2012.630 & 1 & 0.369 & 0.007 & $163.6^{*}$ & 0.7 & & & & & \\
\hline $19083+0948$ & HEI807 & 2011.510 & 1 & 1.503 & 0.008 & $206.7^{*}$ & 0.3 & & & & & \\
\hline $19089+3404$ & $\mathrm{COU} 1462 \mathrm{Aa}, \mathrm{Ab}$ & 2011.559 & 1 & 0.280 & 0.010 & $205.1^{*}$ & 1.2 & & & Doc2003e & 0.06 & -2.7 \\
\hline $19089+3404$ & $\mathrm{COU} 1462 \mathrm{Aa}, \mathrm{Ab}$ & 2012.627 & 1 & 0.244 & 0.007 & $208.6^{*}$ & 0.8 & & & Doc2003e & 0.01 & -1.8 \\
\hline $19093+1138$ & HDS2716 & 2011.576 & 1 & - & - & - & - & & $\mathrm{NR}$ & & & \\
\hline $19095+2303$ & TDS971 & 2012.605 & 1 & 1.148 & 0.007 & $48.2^{*}$ & 0.3 & & & & & \\
\hline $19098-0017$ & A 863 & 2011.521 & 1 & 0.335 & 0.026 & $156.9^{*}$ & 0.7 & & & & & \\
\hline $19100+3821$ & A 263 & 2011.557 & 2 & 1.634 & 0.015 & $216.1^{*}$ & 0.4 & & & & & \\
\hline $19100+3821$ & A 263 & 2011.557 & 2 & 1.649 & 0.024 & $217.2^{*}$ & 0.3 & & & & & \\
\hline $19103+2820$ & COU723 & 2012.605 & 1 & 0.202 & 0.007 & 10.6 & 0.6 & & & & & \\
\hline $19103+3044$ & COU1020 & 2011.557 & 2 & 0.973 & 0.021 & $99.0^{*}$ & 0.9 & & & & & \\
\hline $19104+2030$ & A150 & 2012.556 & 1 & 0.423 & 0.018 & 121.1 & 3.0 & & & & & \\
\hline $19104+2320$ & COU120 & 2011.573 & 1 & 0.117 & 0.047 & $51.4^{*}$ & 7.4 & & & & & \\
\hline $19104+2320$ & COU120 & 2012.635 & 1 & 0.125 & 0.049 & $52.2^{*}$ & 7.4 & & & & & \\
\hline $19108+3726$ & COU1799 & 2012.698 & 1 & 1.430 & 0.007 & 12.9 & 0.3 & & & & & \\
\hline $19108+4123$ & TDT1277 & 2012.635 & 1 & 1.080 & 0.007 & $186.3^{*}$ & 0.4 & & & & & \\
\hline $19108+4720$ & KOI98 & 2012.586 & 1 & 0.247 & 0.007 & $142.1^{*}$ & 0.9 & & & & & \\
\hline $19108+4720$ & KOI98 & 2012.589 & 1 & 0.261 & 0.007 & 145.8 & 0.3 & 6.91 & & & & \\
\hline $19110-0726$ & A95 & 2011.521 & 1 & 0.281 & 0.007 & $26.5^{*}$ & 1.1 & & & Doc2017f & 0.03 & -5.7 \\
\hline $19120+3925$ & YSC114 & 2012.586 & 1 & 0.617 & 0.007 & 313.5 & 0.3 & 3.86 & & & & \\
\hline
\end{tabular}




\begin{tabular}{|c|c|c|c|c|c|c|c|c|c|c|c|c|}
\hline WDS & Name & Epoch & Bin. & $\begin{array}{c}\rho \\
(")\end{array}$ & $\begin{array}{l}\sigma_{\rho} \\
(")\end{array}$ & $\begin{array}{c}\theta \\
\left({ }^{\circ}\right)\end{array}$ & $\begin{array}{l}\sigma_{\theta} \\
\left(^{\circ}\right)\end{array}$ & $\Delta m$ & Notes & Orbit & $\begin{array}{r}\Delta \rho(\mathrm{O}-\mathrm{C}) \\
(")\end{array}$ & $\begin{array}{r}\Delta \theta(\mathrm{O}-\mathrm{C}) \\
\left(^{\circ}\right)\end{array}$ \\
\hline $19120+3925$ & YSC114 & 2012.589 & 1 & 0.556 & 0.007 & $317.6^{*}$ & 0.3 & & & & & \\
\hline $19121+0237$ & BU1204 & 2011.510 & 1 & 0.221 & 0.011 & 2.8 & 0.4 & & & & & \\
\hline $19121+0237$ & BU1204 & 2011.527 & 1 & 0.204 & 0.021 & 0.2 & 0.7 & & & & & \\
\hline $19121+0237$ & BU1204 & 2011.527 & 1 & 0.205 & 0.010 & 2.6 & 0.5 & & & & & \\
\hline $19121+0254$ & AST1 & 2011.510 & 1 & 0.252 & 0.013 & 187.5 & 2.8 & & & AST2016 & 0.05 & 8.7 \\
\hline $19127+1324$ & HEI399 & 2011.576 & 1 & 1.791 & 0.009 & $306.2^{*}$ & 0.3 & & & & & \\
\hline $19127+2435$ & HDS2720 & 2012.687 & 1 & - & - & - & - & & NR & & & \\
\hline $19127+2435$ & $\mathrm{~A} 264 \mathrm{AB}$ & 2012.605 & 1 & 3.570 & 0.018 & 291.0 & 2.3 & 4.08 & & Izm2019 & 0.17 & 2.1 \\
\hline $19127+2435$ & $\mathrm{~A} 264 \mathrm{AB}$ & 2012.687 & 1 & 3.443 & 0.017 & 286.4 & 3.3 & 3.73 & & Izm2019 & 0.05 & -2.5 \\
\hline $19127+2545$ & TDT1295 & 2012.709 & 1 & 0.811 & 0.007 & $137.5^{*}$ & 0.3 & & & & & \\
\hline $19131+2154$ & A153 & 2012.605 & 1 & 0.694 & 0.007 & $265.1^{*}$ & 0.3 & & & & & \\
\hline $19131+2154$ & A153 & 2011.557 & 2 & 0.635 & 0.016 & $266.3^{*}$ & 1.3 & & & & & \\
\hline $19132+3420$ & COU1616 & 2012.627 & 1 & 0.286 & 0.013 & 129.8 & 3.9 & & & & & \\
\hline $19133-0817$ & RST4622 & 2011.521 & 1 & 0.235 & 0.008 & 181.8 & 1.3 & & & & & \\
\hline $19134+2926$ & COU1157 & 2011.573 & 1 & 0.208 & 0.009 & $96.5^{*}$ & 0.9 & & & & & \\
\hline $19136+2012$ & HU335 & 2012.682 & 1 & 0.488 & 0.007 & $245.1^{*}$ & 0.7 & & & & & \\
\hline $19140+4005$ & COU2199 & 2011.573 & 1 & 0.942 & 0.007 & $351.5^{*}$ & 0.4 & & & & & \\
\hline $19141+6707$ & HDS2722 & 2011.516 & 1 & 0.169 & 0.040 & 24.5 & 8.0 & & & & & \\
\hline $19147+3258$ & COU1463 & 2012.635 & 1 & 0.622 & 0.007 & $207.3^{*}$ & 0.5 & & & & & \\
\hline $19150+3539$ & COU1617 & 2012.641 & 1 & 0.925 & 0.007 & $150.2^{*}$ & 0.3 & & & & & \\
\hline $19159+2727$ & STT371 & 2012.714 & 1 & 0.886 & 0.007 & $160.9^{*}$ & 0.3 & & & & & \\
\hline $19162+2817$ & STF2491 & 2011.726 & 1 & 1.362 & 0.007 & $229.2^{*}$ & 0.7 & & & & & \\
\hline $19162+4505$ & COU2521 & 2011.576 & 1 & 0.564 & 0.007 & $277.1^{*}$ & 0.9 & & & & & \\
\hline $19163+4018$ & COU2280 & 2012.682 & 1 & 0.282 & 0.007 & $89.4^{*}$ & 0.5 & & & & & \\
\hline $19172-0323$ & A100 & 2011.527 & 1 & 0.734 & 0.009 & $317.7^{*}$ & 0.3 & & & & & \\
\hline $19174+0115$ & A 2267 & 2011.527 & 1 & 0.426 & 0.007 & 173.3 & 0.3 & & & & & \\
\hline $19177+3654$ & TDT1357 & 2012.684 & 1 & 0.545 & 0.007 & $280.8^{*}$ & 0.3 & & & & & \\
\hline $19178+4759$ & TDT1358 & 2012.641 & 1 & - & - & - & - & & NR & & & \\
\hline $19178+5950$ & HDS2729 & 2011.516 & 1 & 0.143 & 0.040 & 133.4 & 8.0 & & & & & \\
\hline $19180+2012$ & COU321 & 2011.573 & 1 & 0.171 & 0.040 & 173.6 & 8.0 & & & Doc2003e & 0.06 & 9.4 \\
\hline $19180+2012$ & COU321 & 2011.573 & 1 & - & - & - & - & & NR & & & \\
\hline $19180+2012$ & COU321 & 2012.605 & 1 & 0.188 & 0.040 & $144.7^{*}$ & 8.0 & & & Doc2003e & 0.06 & -17.4 \\
\hline $19181-0525$ & HDS2731AB & 2011.521 & 1 & - & - & - & - & & NR & & & \\
\hline $19181-0525$ & HDS2731AC & 2011.521 & 2 & 7.294 & 0.036 & $306.9^{*}$ & 0.3 & & & & & \\
\hline $19188+1736$ & HU337 & 2011.527 & 1 & 0.199 & 0.040 & $83.1^{*}$ & 8.0 & & & & & \\
\hline $19189+7648$ & HDS2732 & 2011.516 & 1 & 0.725 & 0.015 & $85.0^{*}$ & 0.6 & & & & & \\
\hline $19192+1229$ & HEI570 & 2011.510 & 1 & 0.682 & 0.047 & $222.8^{*}$ & 0.3 & & & & & \\
\hline $19192+2844$ & VBS28 & 2012.632 & 1 & 2.032 & 0.010 & $94.6^{*}$ & 0.3 & & & & & \\
\hline $19192+2844$ & VBS28 & 2012.684 & 1 & 2.028 & 0.010 & $93.7^{*}$ & 0.3 & & & & & \\
\hline $19194+1943$ & COU421 & 2012.627 & 1 & 0.130 & 0.040 & 152.4 & 8.0 & & & & & \\
\hline $19196+4035$ & COU2398 & 2011.559 & 1 & 0.169 & 0.040 & $95.9^{*}$ & 8.0 & & & & & \\
\hline $19201+2849$ & COU1022 & 2012.687 & 1 & 0.151 & 0.040 & 142.1 & 8.0 & & & & & \\
\hline $19201+2849$ & COU1022 & 2012.728 & 1 & 0.180 & 0.040 & $143.8^{*}$ & 8.0 & & & & & \\
\hline $19203+2831$ & TDS982 & 2012.635 & 1 & 0.768 & 0.007 & $298.4^{*}$ & 0.3 & & & & & \\
\hline $19204+2631$ & COU1023 & 2012.728 & 1 & 0.262 & 0.007 & $144.9^{*}$ & 1.3 & & & & & \\
\hline $19205+3831$ & KOI300 & 2012.632 & 1 & - & - & - & - & & NR & & & \\
\hline $19205+3831$ & KOI300 & 2012.627 & 1 & - & - & - & - & & NR & & & \\
\hline $19205+3831$ & KOI300 & 2012.586 & 1 & 0.153 & 0.040 & 113.4 & 8.0 & & & & & \\
\hline
\end{tabular}




\begin{tabular}{|c|c|c|c|c|c|c|c|c|c|c|c|c|}
\hline WDS & Name & Epoch & Bin. & $\begin{array}{c}\rho \\
(")\end{array}$ & $\begin{array}{l}\sigma_{\rho} \\
(")\end{array}$ & $\begin{array}{c}\theta \\
\left(^{\circ}\right)\end{array}$ & $\begin{array}{l}\sigma_{\theta} \\
\left(^{\circ}\right)\end{array}$ & $\Delta m$ & Notes & Orbit & $\begin{array}{r}\Delta \rho(\mathrm{O}-\mathrm{C}) \\
(")\end{array}$ & $\begin{array}{r}\Delta \theta(\mathrm{O}-\mathrm{C}) \\
\left({ }^{\circ}\right)\end{array}$ \\
\hline $19213+5543$ & A1395 & 2011.516 & 1 & - & - & - & - & & NR & & & \\
\hline $19218+7708$ & HDS2740 & 2011.516 & 1 & 0.274 & 0.007 & 21.1 & 2.4 & & & & & \\
\hline $19220+2230$ & BU141 & 2011.745 & 1 & 0.911 & 0.007 & $81.8^{*}$ & 0.6 & & & & & \\
\hline $19220+7037$ & HDS2741 & 2011.516 & 1 & 0.211 & 0.007 & $15.1^{*}$ & 5.2 & & & & & \\
\hline $19226+2912$ & TDT1407 & 2012.641 & 1 & 0.937 & 0.011 & $166.6^{*}$ & ${ }^{*} 0.3$ & & & & & \\
\hline $19227+2336$ & TDT1409 & 2012.632 & 1 & 0.892 & 0.007 & $188.7^{*}$ & 0.3 & & & & & \\
\hline $19237+2822$ & COU1024 & 2012.641 & 1 & 0.430 & 0.010 & $237.9^{*}$ & 0.7 & & & & & \\
\hline $19238+3832$ & COU2201 & 2011.573 & 1 & 0.213 & 0.007 & $139.9^{*}$ & 1.6 & & & & & \\
\hline $19240+2433$ & HDS2750 & 2011.559 & 1 & 0.355 & 0.007 & 58.3 & 2.4 & & & & & \\
\hline $19240+2437$ & TDS988AB & 2012.605 & 1 & 0.167 & 0.037 & 141.0 & 4.9 & & & & & \\
\hline $19240+2437$ & TDS988AB & 2012.605 & 1 & 0.207 & 0.037 & 149.4 & 4.3 & 0.36 & & & & \\
\hline $19240+2437$ & TDS988AC & 2012.605 & 1 & 3.279 & 0.016 & 55.6 & 0.3 & & $\mathrm{ND}$ & & & \\
\hline $19240+2437$ & TDS988AC & 2012.627 & 1 & 3.305 & 0.017 & $56.6^{*}$ & 0.3 & & $\mathrm{ND}$ & & & \\
\hline $19243+3156$ & COU1160 & 2012.728 & 1 & 0.546 & 0.007 & $195.2^{*}$ & 0.8 & & & & & \\
\hline $19244+1656$ & HDS2753 & 2011.576 & 1 & 0.446 & 0.015 & $319.1^{*}$ & 0.9 & & & & & \\
\hline $19247+1005$ & HDS2756 & 2011.745 & 1 & 0.187 & 0.040 & 6.6 & 8.0 & & & & & \\
\hline $19252+3928$ & COU2202 & 2011.576 & 1 & 1.373 & 0.014 & $248.9^{*}$ & ${ }^{*} 0.8$ & & & & & \\
\hline $19264+2241$ & GII50 & 2012.641 & 1 & 0.396 & 0.010 & $184.8^{*}$ & ${ }^{*} 0.3$ & & $\mathrm{ND}$ & & & \\
\hline $19264+2241$ & GII50 & 2012.682 & 1 & 0.385 & 0.012 & 187.8 & 2.0 & & ND & & & \\
\hline $19254+2619$ & COU819 & 2011.576 & 1 & 2.076 & 0.023 & $184.5^{*}$ & 0.3 & & & & & \\
\hline $19269+1204$ & A1181 & 2011.745 & 1 & 0.700 & 0.007 & $200.1^{*}$ & 0.5 & & & & & \\
\hline $19269+1818$ & HDS2764 & 2011.745 & 1 & - & - & - & - & & NR & & & \\
\hline $19269+2245$ & CON37AB & 2012.605 & 2 & 1.556 & 0.036 & $325.3^{*}$ & 3.3 & 2.1 & & & & \\
\hline $19269+2245$ & CON37AB & 2012.687 & 2 & 1.484 & 0.026 & 321.9 & 3.1 & 2.05 & & & & \\
\hline $19269+2245$ & CON37AB & 2012.693 & 2 & 1.480 & 0.037 & $325.4^{*}$ & 2.3 & 1.8 & & & & \\
\hline $19269+2245$ & CON37AB & 2012.693 & 2 & 1.592 & 0.035 & 324.9 & 3.3 & 1.75 & & & & \\
\hline $19269+2245$ & CON37AB & 2012.717 & 2 & 1.536 & 0.028 & 326.0 & 3.2 & & & & & \\
\hline $19269+2245$ & CON37AB & 2012.717 & 1 & 1.600 & 0.038 & $324.9^{*}$ & 0.3 & & & & & \\
\hline $19269+2245$ & CON37A,CD & 2012.693 & 2 & 10.39 & 0.08 & 159.8 & 3.0 & 3.2 & & & & \\
\hline $19269+2245$ & CON37CD & 2012.641 & 1 & - & - & - & - & & NR & & & \\
\hline $19269+2245$ & CON37CD & 2012.693 & 1 & 0.590 & 0.007 & 147.3 & 0.3 & 1.12 & & & & \\
\hline $19272+1626$ & HDS2765 & 2011.745 & 1 & 0.260 & 0.008 & $181.8^{*}$ & 0.5 & & & & & \\
\hline $19274+2510$ & COU1026 & 2011.576 & 1 & 1.979 & 0.010 & $13.0^{*}$ & 0.3 & & & & & \\
\hline $19287+1442$ & HEI271 & 2011.745 & 1 & 1.382 & 0.007 & $222.6^{*}$ & 0.3 & & & & & \\
\hline $19288+2305$ & A160 & 2011.742 & 1 & 0.179 & 0.040 & 102.1 & 8.0 & & & & & \\
\hline $19290+1515$ & A1651 & 2011.740 & 1 & 0.209 & 0.007 & $254.7^{*}$ & 3.1 & & & & & \\
\hline $19300+2918$ & COU1318 & 2011.742 & 1 & 0.398 & 0.007 & $200.3^{*}$ & ${ }^{*} 1.4$ & 0.40 & & & & \\
\hline $19302+5639$ & A712 & 2011.516 & 1 & 0.107 & 0.040 & 72.2 & 8.0 & & & USN2006b & 0.01 & -1.6 \\
\hline $19303+4204$ & COU2401 & 2012.714 & 1 & 0.976 & 0.018 & $191.4^{*}$ & 0.4 & & & & & \\
\hline $19306+2817$ & COU1161 & 2011.559 & 1 & 0.232 & 0.007 & 179.1 & 8.2 & & & & & \\
\hline $19306+2817$ & COU1161 & 2011.573 & 1 & 0.109 & 0.063 & 138.1 & 7.2 & & & & & \\
\hline $19310+0429$ & A366 & 2011.521 & 1 & 0.506 & 0.007 & $305.5^{*}$ & 0.3 & & & & & \\
\hline $19310+4210$ & COU2403 & 2011.573 & 1 & 0.345 & 0.007 & $32.2^{*}$ & 0.8 & & & & & \\
\hline $19312+2201$ & A161 & 2011.559 & 1 & - & - & - & - & & NR & & & \\
\hline $19316+1747$ & STF2536 & 2011.742 & 1 & 1.796 & 0.009 & $117.6^{*}$ & 0.3 & & & Izm2019 & -0.02 & -1.2 \\
\hline $19317+3040$ & TDT1520 & 2012.635 & 1 & 1.183 & 0.007 & $66.6^{*}$ & 0.3 & & & & & \\
\hline $19325+4358$ & A585 & 2012.630 & 1 & 1.847 & 0.009 & $291.4^{*}$ & 0.3 & & & & & \\
\hline $19326+3649$ & COU2204 & 2011.740 & 1 & 0.250 & 0.007 & $229.5^{*}$ & ${ }^{*} 1.1$ & & & & & \\
\hline $19330+0546$ & A367 & 2011.756 & 1 & 0.999 & 0.007 & $306.2^{*}$ & 0.3 & & & Izm2019 & 0.05 & 1.3 \\
\hline $19331+3254$ & HU948AB & 2011.740 & 1 & 0.218 & 0.011 & $157.2^{*}$ & ${ }^{*} 1.2$ & & & & & \\
\hline $19333+2629$ & COU1028 & 2011.742 & 1 & 0.280 & 0.007 & $64.4^{*}$ & 0.6 & 0.60 & & & & \\
\hline $19333+3329$ & HO108 & 2011.742 & 1 & 0.210 & 0.015 & $193.3^{*}$ & ${ }^{*} 1.9$ & & & & & \\
\hline $19334-0602$ & RST4632 & 2011.756 & 1 & 0.716 & 0.007 & $53.1^{*}$ & 0.3 & & & & & \\
\hline $19334+3551$ & COU1941 & 2012.630 & 1 & 0.516 & 0.009 & $221.2^{*}$ & 0.3 & & & & & \\
\hline $19334+4111$ & COU2404AB & 2012.630 & 1 & 1.400 & 0.007 & $155.6^{*}$ & 0.3 & & & & & \\
\hline $19334+4111$ & COU2404AB & 2012.630 & 2 & 1.426 & 0.018 & 155.9 & 0.7 & & & & & \\
\hline $19334+4111$ & COU2404AB & 2012.630 & 2 & 1.420 & 0.015 & 156.1 & 0.5 & & & & & \\
\hline $19334+4111$ & COU2404AB & 2012.630 & 2 & 1.389 & 0.015 & 155.9 & 0.3 & & & & & \\
\hline $19334+4111$ & COU2404AC & 2012.630 & 2 & 8.398 & 0.042 & $355.9^{*}$ & 0.3 & & & & & \\
\hline
\end{tabular}




\begin{tabular}{|c|c|c|c|c|c|c|c|c|c|c|c|c|}
\hline WDS & Name & Epoch & Bin. & $\begin{array}{c}\rho \\
(")\end{array}$ & $\begin{array}{l}\sigma_{\rho} \\
(")\end{array}$ & $\begin{array}{c}\theta \\
\left({ }^{\circ}\right)\end{array}$ & $\begin{array}{l}\sigma_{\theta} \\
\left(^{\circ}\right)\end{array}$ & $\Delta m$ & Notes & Orbit & $\begin{array}{r}\Delta \rho(\mathrm{O}-\mathrm{C}) \\
(")\end{array}$ & $\begin{array}{r}\Delta \theta(\mathrm{O}-\mathrm{C}) \\
\left({ }^{\circ}\right)\end{array}$ \\
\hline $19342+0747$ & HDS2777 & 2011.521 & 1 & 0.166 & 0.040 & $241.6^{*}$ & 8.0 & & & & & \\
\hline $19346+1808$ & STT375 & 2011.748 & 1 & 0.621 & 0.013 & $188.2^{*}$ & 0.6 & & & Zir2015a & 0.02 & 1.9 \\
\hline $19347+2854$ & COU1031AB & 2011.510 & 1 & 1.827 & 0.016 & 60.2 & 0.3 & & & & & \\
\hline $19347+2854$ & COU1031AB & 2011.559 & 1 & 1.829 & 0.020 & 60.3 & 0.6 & & & & & \\
\hline $19347+2854$ & COU1031AB & 2012.635 & 1 & 1.790 & 0.039 & $59.7^{*}$ & 0.8 & & & & & \\
\hline $19347+2854$ & GII33Aa,Ab & 2011.510 & 1 & 0.297 & 0.011 & $205.9^{*}$ & 3.9 & & & & & \\
\hline $19347+2854$ & GII33Aa,Ab & 2011.559 & 1 & 0.364 & 0.010 & 207.1 & 0.9 & & & & & \\
\hline $19347+2854$ & GII33Aa,Ab & 2012.635 & 1 & 0.262 & 0.046 & $199.3^{*}$ & 4.3 & & & & & \\
\hline $19348+3751$ & COU2205 & 2011.759 & 1 & 0.306 & 0.007 & $265.3^{*}$ & 0.3 & & & & & \\
\hline $19350+2328$ & A162 & 2012.635 & 1 & 0.153 & 0.040 & 294.0 & 8.0 & & & Mdz2017 & 0.00 & 9.5 \\
\hline $19350+2947$ & A368 & 2012.635 & 1 & 0.506 & 0.007 & $154.2^{*}$ & 0.6 & & & & & \\
\hline $19355+1126$ & BU53 & 2011.745 & 1 & 1.660 & 0.012 & $245.9^{*}$ & 0.3 & & & & & \\
\hline $19356+4002$ & A1400 & 2011.726 & 1 & 0.328 & 0.013 & $121.7^{*}$ & 0.4 & & & USN2002 & 0.08 & -18.7 \\
\hline $19358+2316$ & A163 & 2011.573 & 1 & 0.244 & 0.007 & $190.7^{*}$ & 1.3 & & & Sca2019d & 0.02 & $-7.2^{Q}$ \\
\hline $19360+1416$ & HEI272 & 2011.510 & 1 & - & - & - & - & & NR & & & \\
\hline $19371+4013$ & WSI5 & 2011.726 & 1 & 0.165 & 0.040 & 17.5 & 8.0 & & & & & \\
\hline $19376+2911$ & COU1320 & 2011.740 & 1 & 1.681 & 0.008 & $30.0^{*}$ & 0.3 & & & & & \\
\hline $19379+0130$ & HEI176 & 2011.510 & 1 & 0.227 & 0.007 & $327.5^{*}$ & 0.3 & & & & & \\
\hline $19379+0916$ & J801 & 2011.751 & 1 & 1.590 & 0.008 & $77.3^{*}$ & 0.3 & & & & & \\
\hline $19379+1836$ & HDS2785 & 2011.521 & 1 & 0.182 & 0.040 & 182.0 & 8.0 & & & & & \\
\hline $19379+2248$ & A164 & 2011.745 & 1 & 0.193 & 0.040 & $242.8^{*}$ & 8.0 & & & & & \\
\hline $19379+4312$ & HDS2784 & 2011.759 & 1 & 0.356 & 0.013 & 169.2 & 3.1 & & & & & \\
\hline $19380+1614$ & A1656 & 2011.510 & 1 & 0.224 & 0.026 & $176.0^{*}$ & 3.3 & & & & & \\
\hline $19382+0725$ & TDT1596AB & 2011.751 & 1 & - & - & - & - & & NR & & & \\
\hline $19382+0725$ & TDT1598AB & 2011.751 & 1 & 0.476 & 0.007 & $148.8^{*}$ & 0.3 & & & & & \\
\hline $19382+0725$ & TDT1596AC & 2011.751 & 1 & 2.508 & 0.043 & 122.0 & 0.3 & & $\mathrm{ND}$ & & & \\
\hline $19388+2307$ & HDS2788 & 2011.576 & 1 & 0.325 & 0.012 & $235.8^{*}$ & 4.8 & & & & & \\
\hline $19389+3514$ & HU953 & 2011.740 & 1 & 0.278 & 0.007 & $40.4^{*}$ & 1.2 & & & $\operatorname{Lin} 2017 \mathrm{c}$ & -0.03 & -0.2 \\
\hline $19390+1842$ & COU213 & 2011.745 & 1 & 1.134 & 0.007 & $183.1^{*}$ & 0.3 & & & & & \\
\hline $19394+2215$ & STF2556 & 2011.740 & 1 & 0.368 & 0.007 & $348.8^{*}$ & 0.8 & & & Ari1999 & 0.00 & -0.1 \\
\hline $19394+2215$ & STF2556 & 2011.740 & 1 & 0.371 & 0.007 & 348.6 & 0.3 & & & Ari1999 & 0.01 & -0.3 \\
\hline $19394+2215$ & STF2556 & 2012.755 & 1 & 0.348 & 0.015 & $347.5^{*}$ & 0.8 & & & Ari1999 & -0.02 & -0.0 \\
\hline $19397+3359$ & HO111 & 2012.755 & 1 & 1.290 & 0.007 & $17.5^{*}$ & 0.3 & & & & & \\
\hline $19397+3359$ & HO111 & 2012.755 & 1 & 1.296 & 0.007 & 17.8 & 0.3 & 3.19 & & & & \\
\hline $19400-0306$ & RST5141 & 2011.756 & 1 & 0.263 & 0.007 & 114.4 & 3.9 & & & & & \\
\hline $19404+3118$ & COU1164 & 2011.759 & 1 & 0.362 & 0.007 & $331.4^{*}$ & 0.4 & & & & & \\
\hline $19404+3121$ & COU1163 & 2012.755 & 1 & 0.143 & 0.040 & $165.7^{*}$ & 8.0 & & & & & \\
\hline $19404+3121$ & COU1163 & 2011.576 & 1 & 0.170 & 0.040 & $169.2^{*}$ & 8.0 & & & & & \\
\hline $19409+1523$ & HEI74 & 2011.748 & 1 & 0.836 & 0.007 & $111.5^{*}$ & 0.5 & & & & & \\
\hline $19410+0042$ & TDT1631 & 2011.753 & 1 & - & - & - & - & & NR & & & \\
\hline $19411+1349$ & KUI93 & 2011.510 & 1 & 0.141 & 0.040 & 319.5 & 8.0 & & & Tok2021 & -0.03 & 5.0 \\
\hline $19413+3043$ & BU145 & 2011.723 & 1 & 0.851 & 0.009 & $271.3^{*}$ & 0.3 & & & & & \\
\hline $19414+0813$ & HEI814 & 2011.745 & 1 & 1.473 & 0.007 & $83.7^{*}$ & 0.3 & & & & & \\
\hline $19415+2319$ & A2789 & 2011.723 & 1 & 0.755 & 0.007 & $278.6^{*}$ & 0.3 & & & & & \\
\hline $19420+4015$ & KUI94 & 2011.573 & 1 & 0.463 & 0.007 & $160.5^{*}$ & 0.3 & & & Sod1999 & 0.02 & 0.2 \\
\hline $19424+1515$ & HDS2795 & 2011.745 & 1 & 0.190 & 0.040 & $194.5^{*}$ & 8.0 & & & & & \\
\hline $19426+1150$ & STT380 & 2011.576 & 1 & 0.403 & 0.007 & $72.8^{*}$ & 1.4 & & & & & \\
\hline $19428+1711$ & HU343 & 2011.745 & 1 & - & - & - & - & & NR & & & \\
\hline $19429+2803$ & $\mathrm{~A} 273$ & 2011.723 & 1 & 1.742 & 0.009 & $153.6^{*}$ & 0.3 & & & & & \\
\hline $19429+2822$ & HDS2797 & 2012.635 & 1 & - & - & - & - & & $\mathrm{NR}$ & & & \\
\hline $19432+0117$ & RST5463 & 2011.751 & 1 & 2.397 & 0.012 & $106.5^{*}$ & 0.5 & & & & & \\
\hline $19432+1736$ & COU323 & 2011.751 & 1 & 1.127 & 0.007 & $301.4^{*}$ & 0.3 & & & & & \\
\hline $19439-0034$ & RST5142 & 2011.756 & 1 & 1.457 & 0.007 & $76.8^{*}$ & 0.3 & & & & & \\
\hline $19445+4350$ & COU2633 & 2011.759 & 1 & 0.531 & 0.009 & 158.0 & 0.4 & & & & & \\
\hline
\end{tabular}




\begin{tabular}{|c|c|c|c|c|c|c|c|c|c|c|c|c|}
\hline WDS & Name & Epoch & Bin. & $\begin{array}{c}\rho \\
(")\end{array}$ & $\begin{array}{l}\sigma_{\rho} \\
(")\end{array}$ & $\begin{array}{c}\theta \\
\left({ }^{\circ}\right)\end{array}$ & $\begin{array}{l}\sigma_{\theta} \\
\left({ }^{\circ}\right)\end{array}$ & $\Delta m$ & Notes & Orbit & $\begin{array}{r}\Delta \rho(\mathrm{O}-\mathrm{C}) \\
(")\end{array}$ & $\begin{array}{r}\Delta \theta(\mathrm{O}-\mathrm{C}) \\
\left({ }^{\circ}\right)\end{array}$ \\
\hline $19448+1057$ & HDS2802 & 2011.745 & 1 & 1.063 & 0.007 & 13.1 & 0.3 & & & & & \\
\hline $19449+1047$ & STF2570AB,C & 2011.510 & 1 & 4.252 & 0.021 & $276.8^{*}$ & 0.3 & & & & & \\
\hline $19449+1047$ & AGC10 & 2011.510 & 1 & 0.212 & 0.007 & 140.7 & 2.4 & & & & & \\
\hline $19450+1551$ & HDS2803 & 2011.753 & 1 & - & - & - & - & & $\mathrm{NR}$ & & & \\
\hline $19452+1823$ & TDT1708 & 2011.748 & 1 & 0.979 & 0.011 & $197.4^{*}$ & 0.4 & & & & & \\
\hline $19458+2710$ & KUI95 & 2011.527 & 1 & 2.083 & 0.010 & $58.2^{*}$ & 0.3 & & & Izm2019 & 0.00 & 0.9 \\
\hline $19458+2710$ & KUI95 & 2012.570 & 1 & 2.100 & 0.015 & 57.9 & 0.3 & & & Izm2019 & -0.04 & 0.2 \\
\hline $19458+2710$ & KUI95 & 2012.570 & 1 & 2.042 & 0.010 & $59.0^{*}$ & 0.3 & & & Izm2019 & -0.01 & 1.3 \\
\hline $19458+2710$ & KUI95 & 2012.570 & 1 & 2.076 & 0.196 & 58.8 & 17.2 & 0.22 & & Izm2019 & -0.07 & 1.1 \\
\hline $19458+2710$ & KUI95 & 2012.583 & 1 & 2.116 & 0.013 & 58.8 & 0.5 & & & Izm2019 & -0.03 & 1.1 \\
\hline $19458+2710$ & KUI95 & 2012.583 & 1 & 2.144 & 0.012 & 59.4 & 0.3 & & & Izm2019 & 0.00 & 1.7 \\
\hline $19458+2710$ & KUI95 & 2012.583 & 1 & 2.123 & 0.011 & 58.7 & 0.5 & & & Izm2019 & -0.02 & 1.0 \\
\hline $19458+2710$ & KUI95 & 2012.583 & 1 & 2.143 & 0.040 & 57.8 & 1.5 & 0.67 & & Izm2019 & 0.00 & 0.1 \\
\hline $19458+2710$ & KUI95 & 2012.583 & 1 & 2.138 & 0.059 & 60.0 & 3.7 & 0.85 & & Izm2019 & -0.00 & 2.3 \\
\hline $19458+2710$ & KUI95 & 2012.583 & 1 & 2.128 & 0.011 & 58.4 & 0.6 & 0.47 & & Izm2019 & -0.01 & 0.7 \\
\hline $19458+2710$ & KUI95 & 2012.583 & 1 & 2.133 & 0.024 & 58.3 & 0.6 & 0.74 & & Izm2019 & -0.01 & 0.6 \\
\hline $19459+3953$ & A1404 & 2012.684 & 1 & 0.181 & 0.040 & $108.0^{*}$ & 8.0 & & & & & \\
\hline $19460+3717$ & COU2284 & 2011.740 & 1 & 0.590 & 0.007 & $329.7^{*}$ & 0.7 & & & & & \\
\hline $19463+1648$ & HEI177 & 2011.510 & 1 & 0.382 & 0.010 & $31.3^{*}$ & 1.5 & & & & & \\
\hline $19464+0418$ & BU1301BC & 2011.510 & 1 & 0.724 & 0.008 & $339.2^{*}$ & 0.7 & & & & & \\
\hline $19465+1924$ & $\mathrm{~J} 1335$ & 2011.753 & 1 & 1.338 & 0.007 & $182.7^{*}$ & 0.3 & & & & & \\
\hline $19466-0123$ & RST5143 & 2011.753 & 1 & 0.178 & 0.040 & 153.5 & 8.0 & & & & & \\
\hline $19466+4346$ & HDS2810 & 2011.573 & 1 & 0.359 & 0.018 & $51.6^{*}$ & 2.9 & & & & & \\
\hline $19466+4346$ & HDS2810 & 2011.573 & 1 & 0.337 & 0.007 & 36.9 & 1.4 & & & & & \\
\hline $19470+1911$ & HDS2812 & 2011.753 & 1 & 0.180 & 0.040 & $358.5^{*}$ & 8.0 & & & & & \\
\hline $19470+2710$ & TDT1740Aa,Ab & 2012.630 & 2 & - & - & - & - & & NR & & & \\
\hline $19471-0809$ & A108 & 2011.756 & 1 & 0.267 & 0.007 & $109.1^{*}$ & 0.9 & & & Doc2017l & -0.04 & 4.5 \\
\hline $19474-0148$ & A2993 & 2011.753 & 1 & - & - & - & - & & NR & & & \\
\hline $19477+1905$ & TDT1760 & 2011.748 & 1 & 1.789 & 0.015 & $78.4^{*}$ & 0.3 & & & & & \\
\hline $19480+1541$ & HEI75 & 2011.742 & 1 & 0.610 & 0.007 & $31.5^{*}$ & 0.6 & 1.30 & & & & \\
\hline $19480+3513$ & BU1473 & 2011.576 & 1 & 1.037 & 0.007 & $194.4^{*}$ & 0.7 & & & & & \\
\hline $19484+4113$ & COU2527 & 2011.759 & 1 & 0.558 & 0.009 & $109.0^{*}$ & 0.3 & & & & & \\
\hline $19484+4117$ & COU2526 & 2011.573 & 1 & 0.176 & 0.089 & $235.4^{*}$ & 17.8 & & & & & \\
\hline $19486+3450$ & HU682 & 2011.740 & 1 & 0.374 & 0.007 & $119.1^{*}$ & 0.8 & & & & & \\
\hline $19487+1504$ & A1658 & 2012.684 & 1 & 0.205 & 0.010 & $92.3^{*}$ & 1.8 & & & Doc $2009 \mathrm{~g}$ & -0.02 & 5.6 \\
\hline $19487+1504$ & A1658 & 2012.684 & 1 & 0.208 & 0.015 & 88.6 & 1.1 & & & Doc $2009 \mathrm{~g}$ & -0.02 & 1.9 \\
\hline $19487+3519$ & STT387 & 2011.560 & 1 & 0.522 & 0.007 & $119.0^{*}$ & 0.6 & & & WSI2006b & -0.00 & -0.8 \\
\hline $19490+1909$ & AGC11 & 2011.726 & 1 & 0.200 & 0.009 & $175.5^{*}$ & 2.0 & & & Mut2010b & -0.03 & 5.1 \\
\hline $19490+4423$ & $\mathrm{~A} 718 \mathrm{BC}$ & 2012.627 & 1 & 0.198 & 0.040 & 26.7 & 8.0 & & & & & \\
\hline $19491+1645$ & HDS2819 & 2011.740 & 1 & 0.185 & 0.040 & $194.9^{*}$ & 8.0 & & & & & \\
\hline $19500+3243$ & ES2371 & 2012.755 & 1 & 2.793 & 0.152 & $179.2^{*}$ & 0.3 & & & & & \\
\hline $19501+2348$ & COU121 & 2012.714 & 1 & 0.367 & 0.007 & $261.4^{*}$ & 2.2 & & & & & \\
\hline $19504+2409$ & COU1034 & 2011.742 & 1 & 0.241 & 0.007 & $209.8^{*}$ & 1.2 & 0.30 & & & & \\
\hline $19506+3715$ & COU2285 & 2012.641 & 1 & 1.492 & 0.009 & $66.8^{*}$ & 0.4 & & & & & \\
\hline $19511+2743$ & HDS2827 & 2011.527 & 1 & - & - & - & - & & NR & & & \\
\hline $19513+0938$ & HDS2829 & 2011.745 & 1 & 0.344 & 0.007 & 113.6 & 0.6 & & & & & \\
\hline $19517+4003$ & COU2531AB & 2011.726 & 1 & - & - & - & - & & $\mathrm{NR}$ & & & \\
\hline $19520+1548$ & TDT1810 & 2011.748 & 1 & 1.356 & 0.010 & 165.2 & 0.3 & & & & & \\
\hline $19523+3417$ & TDT1815 & 2012.630 & 1 & 0.154 & 0.040 & 134.3 & 8.0 & & & & & \\
\hline $19524+3537$ & HU685 & 2011.759 & 1 & 1.607 & 0.008 & $63.1^{*}$ & 0.3 & & & & & \\
\hline $19530+3825$ & COU2532 & 2011.759 & 1 & 0.362 & 0.009 & $155.1^{*}$ & 0.4 & & & & & \\
\hline $19534+2020$ & HDS2833 & 2011.527 & 1 & 0.178 & 0.040 & 135.6 & 8.0 & & & & & \\
\hline $19537-0127$ & RST5145 & 2011.756 & 1 & 0.613 & 0.018 & $190.3^{*}$ & 0.3 & & & & & \\
\hline
\end{tabular}




\begin{tabular}{|c|c|c|c|c|c|c|c|c|c|c|c|c|}
\hline WDS & Name & Epoch & Bin. & $\begin{array}{c}\rho \\
(")\end{array}$ & $\begin{array}{l}\sigma_{\rho} \\
(")\end{array}$ & $\begin{array}{c}\theta \\
\left({ }^{\circ}\right)\end{array}$ & $\begin{array}{l}\sigma_{\theta} \\
\left(^{\circ}\right)\end{array}$ & $\Delta m$ & Notes & Orbit & $\begin{array}{r}\Delta \rho(\mathrm{O}-\mathrm{C}) \\
(")\end{array}$ & $\begin{array}{r}\Delta \theta(\mathrm{O}-\mathrm{C}) \\
\left({ }^{\circ}\right)\end{array}$ \\
\hline $19537+2712$ & TDT1831 & 2012.712 & 1 & 1.726 & 0.009 & $70.0^{*}$ & 0.3 & & & & & \\
\hline $19539+3257$ & COU1626 & 2011.573 & 1 & - & - & - & - & & NR & & & \\
\hline $19540+2616$ & COU1321 & 2011.742 & 1 & 0.314 & 0.008 & $138.0^{*}$ & 1.2 & 0.60 & & & & \\
\hline $19550+4152$ & HO581 & 2012.712 & 1 & 0.115 & 0.040 & 229.7 & 8.0 & & & Sod1999 & -0.04 & 3.1 \\
\hline $19551+3611$ & COU1946 & 2011.742 & 1 & 0.969 & 0.007 & $172.5^{*}$ & 0.4 & & & & & \\
\hline $19553-0644$ & STF2597 & 2011.756 & 1 & 0.620 & 0.007 & $101.3^{*}$ & 0.8 & & & Hrt1996a & -0.01 & 0.4 \\
\hline $19554+2341$ & COU1036 & 2011.740 & 1 & 0.278 & 0.007 & $197.1^{*}$ & 0.6 & & & & & \\
\hline $19554+2341$ & COU1036 & 2012.755 & 1 & 0.278 & 0.007 & $194.2^{*}$ & 0.6 & & & & & \\
\hline $19559+2500$ & COU1037 & 2012.687 & 1 & 0.178 & 0.040 & $153.9^{*}$ & 8.0 & & & Doc2010h & 0.01 & 1.1 \\
\hline $19561+1151$ & J153 & 2011.748 & 1 & 1.835 & 0.009 & $3.7^{*}$ & 0.3 & & & & & \\
\hline $19562+0456$ & HEI575 & 2011.510 & 1 & 1.373 & 0.013 & $237.8^{*}$ & 0.3 & & & & & \\
\hline $19564+2015$ & TDS1025 & 2012.709 & 1 & 1.182 & 0.007 & $170.7^{*}$ & 0.3 & & & & & \\
\hline $19577-0349$ & A2690 & 2011.753 & 1 & 1.430 & 0.007 & $213.5^{*}$ & 0.3 & & & & & \\
\hline $19579+3353$ & COU1805 & 2011.759 & 1 & 0.312 & 0.007 & $62.8^{*}$ & 0.6 & & & & & \\
\hline $19579+3353$ & COU1805 & 2012.709 & 1 & 0.300 & 0.007 & $63.5^{*}$ & 0.5 & & & & & \\
\hline $19580+0456$ & A606 & 2011.745 & 1 & 0.576 & 0.010 & $159.9^{*}$ & 0.4 & & & Sca2016c & 0.04 & 0.6 \\
\hline $19580+3840$ & A1409 & 2011.740 & 1 & 0.445 & 0.009 & $0.8^{*}$ & 0.4 & & & & & \\
\hline $19582-0621$ & HDS2843 & 2011.753 & 1 & 0.316 & 0.017 & $174.2^{*}$ & 1.3 & & & & & \\
\hline $19585+3317$ & STF2606 & 2011.573 & 1 & 0.719 & 0.007 & $145.6^{*}$ & 0.9 & & & Zir2015a & 0.02 & -0.2 \\
\hline $19591+3809$ & COU2411 & 2011.726 & 1 & 0.382 & 0.007 & $45.6^{*}$ & 0.3 & & & & & \\
\hline $19598-0957$ & HO276 & 2011.753 & 1 & - & - & - & - & & NR & & & \\
\hline $20001+0338$ & A2393 & 2011.753 & 1 & 0.198 & 0.040 & 9.8 & 8.0 & & & & & \\
\hline $20002+1454$ & HDS2847 & 2011.573 & 1 & 0.173 & 0.040 & 101.2 & 8.0 & & & & & \\
\hline $20003+1306$ & HDS2849 & 2011.745 & 1 & - & - & - & - & & NR & & & \\
\hline $20003+1757$ & HU352AB & 2011.753 & 1 & 0.180 & 0.040 & $197.8^{*}$ & 8.0 & & & & & \\
\hline $20007+3635$ & HDS2851 & 2011.576 & 1 & 0.367 & 0.007 & 171.2 & 1.1 & & & & & \\
\hline $20008-0749$ & RST4647 & 2011.756 & 1 & 0.463 & 0.007 & $80.8^{*}$ & 0.3 & & & & & \\
\hline $20009-0230$ & RST5150 & 2011.756 & 1 & 0.525 & 0.007 & $121.3^{*}$ & ${ }^{*} 0.4$ & & & & & \\
\hline $20014+1202$ & TDT1917 & 2011.748 & 1 & - & - & - & - & & NR & & & \\
\hline $20015+2439$ & TDS1032 & 2012.728 & 1 & 0.810 & 0.007 & $95.1^{*}$ & 0.3 & & & & & \\
\hline $20016+3435$ & COU1947 & 2011.573 & 1 & 0.249 & 0.016 & $90.6^{*}$ & 0.7 & & & & & \\
\hline $20020+0257$ & HEI818 & 2011.748 & 1 & 2.060 & 0.010 & $24.1^{*}$ & 0.3 & & & & & \\
\hline $20023+2009$ & COU215 & 2012.728 & 1 & 0.304 & 0.007 & $271.4^{*}$ & 1.9 & & & & & \\
\hline $20024+2105$ & HDS2855 & 2012.755 & 1 & 0.140 & 0.040 & 145.7 & 8.0 & & & & & \\
\hline $20032+0444$ & HDS2860 & 2011.745 & 1 & - & - & - & - & & NR & & & \\
\hline $20033+2209$ & OL110AC & 2012.635 & 2 & 8.297 & 0.041 & $331.2^{*}$ & ${ }^{*} 0.3$ & & & & & \\
\hline $20034+3815$ & A1412 & 2012.764 & 1 & 0.753 & 0.007 & $226.0^{*}$ & ${ }^{*} 0.3$ & & & & & \\
\hline $20039+2034$ & COU216 & 2011.723 & 1 & 0.422 & 0.007 & $216.6^{*}$ & 0.3 & & & & & \\
\hline $20041+3957$ & COU2534 & 2011.759 & 1 & 0.191 & 0.040 & $196.5^{*}$ & 8.0 & & & & & \\
\hline $20050+3707$ & COU2212 & 2012.684 & 1 & 0.514 & 0.007 & 17.2 & 0.3 & & & & & \\
\hline $20052+4044$ & A381 & 2012.682 & 1 & 0.779 & 0.007 & $277.3^{*}$ & ${ }^{*} 0.3$ & & & & & \\
\hline $20059+3247$ & COU1807 & 2011.726 & 1 & - & - & - & - & & NR & & & \\
\hline $20063+1954$ & WAK19 & 2012.682 & 1 & 2.018 & 0.014 & 165.4 & 0.6 & & & & & \\
\hline $20063+3148$ & COU1322 & 2011.573 & 1 & 0.115 & 0.045 & 102.8 & 6.6 & & & & & \\
\hline $20063+3148$ & COU1322 & 2011.573 & 1 & 0.104 & 0.047 & 104.8 & 7.6 & & & & & \\
\hline $20067+1256$ & BU428 & 2011.745 & 1 & 0.810 & 0.007 & $354.7^{*}$ & ${ }^{*} 0.3$ & & & & & \\
\hline $20068+0157$ & $\mathrm{~A} 2278 \mathrm{AB}$ & 2011.510 & 1 & 0.921 & 0.007 & $35.0^{*}$ & 0.3 & & & & & \\
\hline $20069+3438$ & COU2124 & 2011.573 & 1 & 0.359 & 0.007 & $34.1^{*}$ & 0.8 & & & & & \\
\hline $20072+1141$ & HEI579 & 2011.751 & 2 & 2.118 & 0.016 & $326.6^{*}$ & 0.3 & & & & & \\
\hline $20073+3534$ & $\mathrm{COU} 2125 \mathrm{Aa}, \mathrm{Ab}$ & 2011.576 & 1 & - & - & - & - & & NR & & & \\
\hline $20075+3535$ & COU2126 & 2012.755 & 1 & 0.680 & 0.007 & $337.4^{*}$ & 0.3 & & & & & \\
\hline $20078+1852$ & TDT2005 & 2011.748 & 1 & - & - & - & - & & NR & & & \\
\hline $20079+1621$ & HEI76 & 2011.510 & 1 & - & - & - & - & & NR & & & \\
\hline
\end{tabular}




\begin{tabular}{|c|c|c|c|c|c|c|c|c|c|c|c|c|}
\hline WDS & Name & Epoch & Bin. & $\begin{array}{c}\rho \\
(")\end{array}$ & $\begin{array}{l}\sigma_{\rho} \\
(")\end{array}$ & $\begin{array}{c}\theta \\
\left({ }^{\circ}\right)\end{array}$ & $\begin{array}{l}\sigma_{\theta} \\
\left({ }^{\circ}\right)\end{array}$ & $\Delta m$ & Notes & Orbit & $\begin{array}{r}\Delta \rho(\mathrm{O}-\mathrm{C}) \\
(")\end{array}$ & $\begin{array}{r}\Delta \theta(\mathrm{O}-\mathrm{C}) \\
\left({ }^{\circ}\right)\end{array}$ \\
\hline $20080+4204$ & A383 & 2012.712 & 1 & 0.345 & 0.007 & $187.8^{*}$ & 0.3 & & & Hrt2013c & -0.00 & $-3.0^{Q}$ \\
\hline $20081+3554$ & TDS1043 & 2012.764 & 1 & 0.921 & 0.007 & $234.6^{*}$ & 0.6 & & & & & \\
\hline $20082+2715$ & COU1167 & 2011.726 & 1 & 2.026 & 0.010 & $238.6^{*}$ & 0.3 & & & & & \\
\hline $20083+3519$ & COU2127 & 2011.759 & 1 & 0.248 & 0.007 & $222.6^{*}$ & 1.6 & & & & & \\
\hline $20083+3519$ & COU2127 & 2012.685 & 1 & 0.298 & 0.007 & $35.2^{*}$ & 1.9 & & & & & \\
\hline $20096+4009$ & COU2414 & 2011.759 & 1 & 0.275 & 0.007 & $158.5^{*}$ & 1.0 & & & & & \\
\hline $20101+2712$ & TDT2040 & 2011.740 & 1 & 1.809 & 0.013 & $254.7^{*}$ & 0.4 & & & & & \\
\hline $20101+2712$ & TDT2040 & 2012.630 & 1 & 1.810 & 0.013 & $255.1^{*}$ & 0.3 & & & & & \\
\hline $20102+2131$ & TDT2042 & 2011.723 & 1 & 0.567 & 0.008 & $358.5^{*}$ & 0.6 & & & & & \\
\hline $20105+2503$ & COU122 & 2011.827 & 1 & 0.635 & 0.007 & $353.2^{*}$ & 0.4 & & & & & \\
\hline $20105+2920$ & $\mathrm{HDS} 2874 \mathrm{Ca}, \mathrm{Cb}$ & 2012.630 & 1 & 0.379 & 0.008 & 71.0 & 5.0 & & & & & \\
\hline $20108+2029$ & HDS2875 & 2011.740 & 1 & 0.306 & 0.010 & $230.9^{*}$ & 4.3 & & & & & \\
\hline $20109+3047$ & HDS 2876 & 2011.740 & 1 & - & - & - & - & & $\mathrm{NR}$ & & & \\
\hline $20110+2928$ & TDT2056 & 2012.725 & 1 & 1.463 & 0.008 & $357.6^{*}$ & 0.3 & & & & & \\
\hline $20113+3338$ & COU1630 & 2012.709 & 1 & 0.115 & 0.040 & 65.8 & 8.0 & & & & & \\
\hline $20118+3936$ & COU2415 & 2012.755 & 1 & 0.324 & 0.007 & $155.2^{*}$ & 1.0 & & & & & \\
\hline $20119+2625$ & HDS2879 & 2012.712 & 1 & 0.293 & 0.007 & $162.1^{*}$ & 0.8 & & & & & \\
\hline $20120+3333$ & COU1631 & 2011.742 & 1 & 0.382 & 0.007 & $327.1^{*}$ & 0.3 & 0.50 & & & & \\
\hline $20120+3429$ & $\mathrm{~A} 282 \mathrm{AB}$ & 2011.742 & 1 & 0.255 & 0.011 & $206.7^{*}$ & 2.2 & & & & & \\
\hline $20120+3436$ & HO120 & 2011.723 & 1 & 1.216 & 0.007 & $110.6^{*}$ & 0.3 & & & & & \\
\hline $20121+0306$ & HEI581 & 2011.510 & 1 & 0.841 & 0.009 & $340.2^{*}$ & 0.5 & & & & & \\
\hline $20129+2913$ & A1203 & 2012.687 & 1 & 0.396 & 0.009 & 176.7 & 1.7 & & & & & \\
\hline $20129+2913$ & A1203 & 2012.687 & 1 & 0.383 & 0.007 & $355.7^{*}$ & 0.8 & & & & & \\
\hline $20135+3526$ & COU2214AB & 2011.573 & 1 & 1.127 & 0.008 & $211.3^{*}$ & 0.3 & & & & & \\
\hline $20135+3526$ & COU2214AB & 2011.759 & 1 & 1.151 & 0.007 & $209.6^{*}$ & 0.3 & & & & & \\
\hline $20135+3526$ & COU2214AB & 2012.685 & 1 & 1.137 & 0.007 & $208.7^{*}$ & 0.3 & & & & & \\
\hline $20135+3526$ & COU2214AB & 2012.709 & 1 & 1.144 & 0.007 & $210.8^{*}$ & 0.3 & & & & & \\
\hline $20135+3526$ & COU2214AB & 2012.709 & 1 & 1.092 & 0.007 & 210.4 & 0.3 & 0.13 & & & & \\
\hline $20135+3526$ & COU2214AC & 2011.573 & 1 & 3.420 & 0.018 & $263.6^{*}$ & 0.3 & & $\mathrm{ND}$ & & & \\
\hline $20135+3526$ & COU2214AC & 2011.576 & 1 & 3.404 & 0.017 & $263.3^{*}$ & 0.3 & & $\mathrm{ND}$ & & & \\
\hline $20137+1302$ & A1668 & 2011.748 & 1 & 1.457 & 0.010 & $340.7^{*}$ & 0.3 & & & & & \\
\hline $20139+4539$ & POP138 & 2012.755 & 1 & 0.545 & 0.007 & $220.9^{*}$ & 0.3 & & & & & \\
\hline $20140+3031$ & COU1475 & 2011.742 & 1 & 0.216 & 0.011 & $75.8^{*}$ & 2.1 & & & & & \\
\hline $20141-0450$ & HDS2883 & 2011.753 & 2 & 0.866 & 0.034 & 137.8 & 1.2 & & & & & \\
\hline $20141-0450$ & HDS2883 & 2011.753 & 2 & 0.952 & 0.055 & 138.4 & 1.4 & & & & & \\
\hline $20144+3501$ & COU2216 & 2012.725 & 1 & 0.554 & 0.007 & $206.2^{*}$ & 0.5 & & & & & \\
\hline $20148+2159$ & HDS2886 & 2011.745 & 1 & 1.012 & 0.007 & $63.6^{*}$ & 0.3 & & & & & \\
\hline $20149+2840$ & HO122 & 2012.764 & 1 & 1.406 & 0.010 & $72.2^{*}$ & 0.3 & & & & & \\
\hline $20151+3742$ & COU2416 & 2012.682 & 1 & 0.293 & 0.011 & $126.1^{*}$ & 0.9 & & & Doc2013g & 0.02 & -0.0 \\
\hline $20151+3742$ & COU2416 & 2011.723 & 1 & 0.297 & 0.007 & $124.5^{*}$ & 0.9 & & & Doc2013g & 0.03 & 1.3 \\
\hline $20155+4030$ & A390 & 2012.000 & 2 & 0.822 & 0.038 & 326.4 & 0.5 & & & & & \\
\hline $20155+4030$ & A390 & 2012.687 & 1 & 0.852 & 0.047 & 326.2 & 0.6 & & & & & \\
\hline $20155+4030$ & A390 & 2012.687 & 1 & 0.867 & 0.025 & 325.3 & 1.2 & & & & & \\
\hline $20155+4030$ & A390 & 2012.687 & 1 & 0.834 & 0.037 & $325.7^{*}$ & 0.3 & & & & & \\
\hline $20157+4339$ & A2095AB & 2012.687 & 1 & 0.241 & 0.007 & $332.7^{*}$ & 0.4 & & & $\operatorname{Lin} 2012 \mathrm{c}$ & -0.03 & $-1.6^{Q}$ \\
\hline $20157+4339$ & A2095A,BC & 2012.687 & 1 & 3.048 & 0.015 & $313.9^{*}$ & 0.3 & & & & & \\
\hline $20163+2033$ & HDS2888 & 2011.745 & 1 & 0.646 & 0.010 & $283.0^{*}$ & 0.9 & & & & & \\
\hline $20164+2309$ & COU218 & 2012.709 & 1 & 0.339 & 0.021 & $218.6^{*}$ & 0.6 & & & & & \\
\hline $20165+2747$ & HDS2889 & 2011.576 & 1 & 0.186 & 0.040 & 122.1 & 8.0 & & & & & \\
\hline $20168+3435$ & TDT2137 & 2012.725 & 1 & 0.613 & 0.007 & $110.1^{*}$ & 0.8 & & & & & \\
\hline $20170+3725$ & HDS2891 & 2011.726 & 1 & 0.914 & 0.007 & $53.3^{*}$ & 0.3 & & & & & \\
\hline $20170+3725$ & A1423AB & 2011.726 & 1 & 4.517 & 0.023 & $128.8^{*}$ & 0.3 & & & & & \\
\hline
\end{tabular}




\begin{tabular}{|c|c|c|c|c|c|c|c|c|c|c|c|c|}
\hline WDS & Name & Epoch & Bin. & $\begin{array}{c}\rho \\
(")\end{array}$ & $\begin{array}{l}\sigma_{\rho} \\
(")\end{array}$ & $\begin{array}{c}\theta \\
\left(^{\circ}\right)\end{array}$ & $\begin{array}{l}\sigma_{\theta} \\
\left(^{\circ}\right)\end{array}$ & $\Delta m$ & Notes & Orbit & $\begin{array}{r}\Delta \rho(\mathrm{O}-\mathrm{C}) \\
(")\end{array}$ & $\begin{array}{r}\Delta \theta(\mathrm{O}-\mathrm{C}) \\
\left({ }^{\circ}\right)\end{array}$ \\
\hline $20176+2622$ & BU984 & 2011.573 & 1 & 0.201 & 0.040 & 153.9 & 8.0 & & & Pop2000a & -0.07 & 29.1 \\
\hline $20176+2622$ & BU984 & 2012.764 & 1 & 0.161 & 0.040 & $159.7^{*}$ & ${ }^{*} 8.0$ & & & Pop2000a & -0.11 & $30.6^{Q}$ \\
\hline $20177+4030$ & HDS2897 & 2011.759 & 1 & 0.205 & 0.015 & 276.3 & 0.9 & & & & & \\
\hline $20177+4030$ & HDS2897 & 2011.759 & 1 & 0.205 & 0.015 & $276.3^{*}$ & * 0.9 & & & & & \\
\hline $20177+4030$ & HDS2897 & 2012.687 & 1 & 0.266 & 0.007 & $273.0^{*}$ & ${ }^{*} 1.9$ & & & & & \\
\hline $20181+2730$ & HDS2898 & 2011.740 & 1 & 0.199 & 0.040 & 168.2 & 8.0 & & & & & \\
\hline $20182+1820$ & HU357 & 2011.748 & 1 & 1.768 & 0.009 & $195.2^{*}$ & ${ }^{*} 0.3$ & & & & & \\
\hline $20182+2912$ & A1205AB & 2012.687 & 1 & 1.188 & 0.007 & $97.0^{*}$ & 0.4 & & & WSI2006b & 0.00 & -0.2 \\
\hline $20187+2001$ & HDS2901 & 2011.745 & 1 & 0.292 & 0.030 & 46.1 & 4.2 & & & & & \\
\hline $20188+3102$ & HDS2902 & 2011.726 & 1 & 1.676 & 0.013 & $295.6^{*}$ & ${ }^{*} 0.3$ & & & & & \\
\hline $20189+3325$ & COU1948 & 2012.712 & 1 & 0.168 & 0.040 & $342.4^{*}$ & ${ }^{*} 8.0$ & & & & & \\
\hline $20189+3817$ & $\mathrm{~A} 1425$ & 2011.573 & 1 & 0.182 & 0.040 & $266.2^{*}$ & * 8.0 & & & & & \\
\hline $20191+3648$ & COU2286 & 2011.742 & 1 & 0.523 & 0.007 & $121.6^{*}$ & * 0.8 & 0.40 & & & & \\
\hline $20195+1533$ & HDS2905 & 2011.510 & 1 & 0.321 & 0.007 & 116.1 & 2.9 & & & & & \\
\hline $20199+3725$ & COU2287 & 2012.764 & 1 & 0.194 & 0.040 & $314.5^{*}$ & * 8.0 & & & & & \\
\hline $20200+1848$ & HU359 & 2011.740 & 1 & 0.194 & 0.040 & 179.2 & 8.0 & & & & & \\
\hline $20201+3700$ & TDT2183AB & 2012.755 & 2 & 1.624 & 0.016 & 192.3 & 0.3 & & & & & \\
\hline $20201+3700$ & TDT2183AC & 2012.755 & 2 & 2.922 & 0.029 & 258.8 & 0.3 & 1.79 & ND & & & \\
\hline $20202+1128$ & HDS2906 & 2011.510 & 1 & 0.577 & 0.016 & $316.9^{*}$ & ${ }^{*} 0.7$ & & & & & \\
\hline $20203+3924$ & A 1427 & 2012.712 & 1 & 0.190 & 0.040 & 132.4 & 8.0 & & & Doc2016h & -0.04 & 1.3 \\
\hline $20205+1634$ & HU360AB & 2011.748 & 1 & - & - & - & - & & NR & & & \\
\hline $20205+1634$ & HU360AC & 2011.748 & 2 & 7.327 & 0.037 & $347.8^{*}$ & ${ }^{*} 0.3$ & & $\mathrm{ND}$ & & & \\
\hline $20205+1634$ & HU360AC & 2011.751 & 2 & 7.324 & 0.037 & $348.2^{*}$ & ${ }^{*} 0.3$ & & $\mathrm{ND}$ & & & \\
\hline $20207+1601$ & YSC70 & 2012.682 & 1 & 0.525 & 0.010 & 155.8 & 0.4 & & & & & \\
\hline $20207+3512$ & COU2218 & 2012.693 & 1 & 0.210 & 0.007 & $88.2^{*}$ & 0.5 & & & & & \\
\hline $20209+3928$ & HO127 & 2012.627 & 1 & 1.914 & 0.010 & $94.0^{*}$ & 0.3 & & & & & \\
\hline $20215+0034$ & A 2280 & 2011.751 & 1 & 0.319 & 0.034 & $31.3^{*}$ & 3.4 & & & & & \\
\hline $20216+1930$ & COU327AB & 2011.510 & 1 & 0.176 & 0.040 & $235.8^{*}$ & $* 8.0$ & & & Doc2008a & 0.02 & -6.9 \\
\hline $20216+1930$ & COU327AB,C & 2011.510 & 1 & 4.088 & 0.020 & 132.2 & 0.3 & & & & & \\
\hline $20219+1336$ & HU1197 & 2011.753 & 1 & 1.186 & 0.007 & $305.2^{*}$ & * 0.3 & & & & & \\
\hline $20219+3017$ & TDT2209 & 2012.627 & 1 & 1.731 & 0.009 & $50.9^{*}$ & 0.3 & & & & & \\
\hline $20227+2837$ & COU1169 & 2011.759 & 1 & 0.243 & 0.007 & $58.6^{*}$ & 0.3 & & & & & \\
\hline $20227+2837$ & COU1169 & 2011.759 & 1 & 0.239 & 0.007 & $59.2^{*}$ & 1.0 & & & & & \\
\hline $20227+2837$ & COU1169 & 2012.709 & 1 & 0.224 & 0.015 & $59.8^{*}$ & 0.6 & & & & & \\
\hline $20230+2020$ & HDS2911 & 2011.745 & 1 & 0.384 & 0.010 & $332.5^{*}$ & * 1.6 & & & & & \\
\hline $20231+2052$ & A 288 & 2011.573 & 1 & 0.181 & 0.040 & $110.3^{*}$ & $* 8.0$ & & & Doc1994d & -0.02 & $-4.1^{Q}$ \\
\hline $20231+2052$ & A 288 & 2012.728 & 1 & 0.196 & 0.040 & 115.6 & 8.0 & & & Doc1994d & -0.00 & $-0.8^{Q}$ \\
\hline $20231+3342$ & COU1949 & 2012.712 & 1 & 0.197 & 0.040 & $83.0^{*}$ & 8.0 & & & & & \\
\hline $20236-0055$ & RST5155 & 2011.751 & 1 & 1.126 & 0.007 & $11.9^{*}$ & 0.3 & & & & & \\
\hline $20236+3854$ & GII51 & 2012.725 & 1 & 3.246 & 0.016 & $355.8^{*}$ & * 0.3 & & $\mathrm{ND}$ & & & \\
\hline $20236+3854$ & GII51 & 2012.728 & 1 & 3.192 & 0.016 & $356.6^{*}$ & * 0.3 & & $\mathrm{ND}$ & & & \\
\hline $20237-0104$ & HDS2913 & 2011.751 & 1 & 0.588 & 0.012 & 4.1 & 0.3 & & & & & \\
\hline $20244+1213$ & A1209AB & 2011.576 & 1 & 1.875 & 0.009 & $324.1^{*}$ & * 0.3 & & & & & \\
\hline $20244+1213$ & WOR33Aa,Ab & 2011.576 & 1 & 0.576 & 0.007 & $133.0^{*}$ & * 0.3 & & & & & \\
\hline $20244+1301$ & HU1198 & 2011.745 & 1 & 0.571 & 0.007 & $31.2^{*}$ & 0.4 & & & & & \\
\hline $20244+2802$ & HDS2918 & 2011.573 & 1 & 0.202 & 0.040 & $218.0^{*}$ & $* 8.0$ & & & & & \\
\hline $20244+2802$ & HDS2918 & 2012.693 & 1 & - & - & - & - & & NR & & & \\
\hline $20244+2802$ & HDS2918 & 2012.728 & 1 & 0.154 & 0.040 & 227.5 & 8.0 & & & & & \\
\hline $20249+1309$ & J841 & 2011.751 & 1 & 0.176 & 0.040 & 179.6 & 8.0 & & & & & \\
\hline $20249+3404$ & A 290 & 2011.827 & 1 & 0.484 & 0.007 & $313.1^{*}$ & * 0.3 & & & & & \\
\hline $20254+4014$ & MLR1 & 2012.685 & 1 & 0.394 & 0.016 & $213.6^{*}$ & * 0.9 & & & & & \\
\hline $20254+4014$ & MLR1 & 2011.759 & 1 & 0.395 & 0.008 & $213.0^{*}$ & * 0.7 & & & & & \\
\hline
\end{tabular}




\begin{tabular}{|c|c|c|c|c|c|c|c|c|c|c|c|c|}
\hline WDS & Name & Epoch & Bin. & $\begin{array}{c}\rho \\
(")\end{array}$ & $\begin{array}{l}\sigma_{\rho} \\
(")\end{array}$ & $\begin{array}{c}\theta \\
\left({ }^{\circ}\right)\end{array}$ & $\begin{array}{l}\sigma_{\theta} \\
\left({ }^{\circ}\right)\end{array}$ & $\Delta m$ & Notes & Orbit & $\begin{array}{r}\Delta \rho(\mathrm{O}-\mathrm{C}) \\
(")\end{array}$ & $\begin{array}{r}\Delta \theta(\mathrm{O}-\mathrm{C}) \\
\left({ }^{\circ}\right)\end{array}$ \\
\hline $20254+4129$ & COU2642 & 2012.693 & 1 & 0.746 & 0.007 & $321.4^{*}$ & * 0.4 & & & & & \\
\hline $20260+3512$ & COU2129 & 2012.685 & 1 & 0.205 & 0.007 & $157.2^{*}$ & * 2.1 & & & & & \\
\hline $20260+3549$ & HDS2922 & 2012.693 & 1 & 0.621 & 0.012 & $142.6^{*}$ & * 0.3 & & & & & \\
\hline $20262+3547$ & COU2130 & 2012.709 & 1 & 0.127 & 0.040 & 49.0 & 8.0 & & & & & \\
\hline $20266-0343$ & RST4666 & 2011.753 & 1 & 0.499 & 0.007 & $199.1^{*}$ & * 0.5 & & & & & \\
\hline $20266+3424$ & TDT2274 & 2011.748 & 1 & - & - & - & - & & NR & & & \\
\hline $20273+3357$ & COU1955 & 2012.712 & 1 & 0.390 & 0.012 & $348.3^{*}$ & * 1.4 & & & & & \\
\hline $20276+4202$ & HDS2928 & 2012.764 & 1 & 0.173 & 0.040 & $14.6^{*}$ & 8.0 & & & & & \\
\hline $20278+3447$ & COU2131 & 2012.728 & 1 & 1.058 & 0.007 & $280.3^{*}$ & * 0.3 & & & & & \\
\hline $20286+3118$ & COU1325 & 2012.712 & 1 & 0.232 & 0.013 & 42.3 & 0.5 & & & & & \\
\hline $20290+0710$ & A610 & 2011.745 & 1 & 0.475 & 0.007 & $79.7^{*}$ & 0.6 & & & Cot2002 & 0.04 & -0.0 \\
\hline $20290+3535$ & COU1959 & 2011.726 & 1 & 0.358 & 0.007 & $324.7^{*}$ & ${ }^{*} 0.3$ & & & & & \\
\hline $20302+2651$ & WOR9 & 2012.627 & 1 & 0.509 & 0.007 & $244.7^{*}$ & * 0.3 & & & Zir2003 & 0.02 & 5.2 \\
\hline $20307+3521$ & HU760AB & 2012.764 & 1 & 0.258 & 0.007 & $67.2^{*}$ & 1.4 & & & & & \\
\hline $20307+3521$ & HU760AC & 2012.764 & 2 & 8.720 & 0.044 & $148.6^{*}$ & * 0.3 & & & & & \\
\hline $20311+1548$ & $\mathrm{~A} 1675$ & 2011.808 & 1 & 0.105 & 0.047 & 324.5 & 7.0 & & & Baz1987d & -0.09 & 7.6 \\
\hline $20311+3333$ & COU1962 & 2011.573 & 1 & 0.215 & 0.007 & $289.2^{*}$ & * 1.2 & & & Tok2021 & -0.02 & $4.4^{Q}$ \\
\hline $20311+3333$ & COU1962 & 2012.627 & 1 & 0.216 & 0.007 & 292.4 & 1.4 & & & Tok2021 & -0.03 & 4.8 \\
\hline $20311+3333$ & COU1962 & 2012.627 & 1 & 0.210 & 0.007 & $107.7^{*}$ & * 0.6 & & & Tok2021 & -0.03 & 0.1 \\
\hline $20320+2548$ & STF2695 & 2011.740 & 1 & 0.375 & 0.007 & $254.3^{*}$ & * 0.3 & & & Alz2005a & -0.04 & -0.6 \\
\hline $20321+1511$ & A1677 & 2011.751 & 1 & 1.283 & 0.007 & $183.7^{*}$ & * 0.3 & & & & & \\
\hline $20328+4418$ & COU2645 & 2012.627 & 1 & 0.248 & 0.007 & $295.2^{*}$ & * 0.8 & & & & & \\
\hline $20328+4418$ & COU2645 & 2011.759 & 1 & 0.248 & 0.007 & 301.1 & 0.3 & & & & & \\
\hline $20329+1357$ & L35CD & 2011.576 & 1 & 0.472 & 0.007 & $156.4^{*}$ & * 0.3 & & & Hrt014b & 0.01 & 1.3 \\
\hline $20329+1357$ & BU670AB & 2011.576 & 1 & 0.821 & 0.007 & $7.5^{*}$ & 0.3 & & & & & \\
\hline $20329+1357$ & BU670AB & 2012.816 & 1 & 0.824 & 0.007 & $7.3^{*}$ & 0.3 & & & & & \\
\hline $20329+3056$ & COU1326 & 2011.742 & 1 & 0.215 & 0.048 & $262.4^{*}$ & * 6.9 & 0.10 & & & & \\
\hline $20331+2324$ & A2792 & 2012.685 & 1 & 0.261 & 0.007 & $127.8^{*}$ & * 0.6 & & & & & \\
\hline $20333+4119$ & HER4 & 2012.627 & 1 & 3.131 & 0.016 & 160.2 & 0.3 & & & & & \\
\hline $20334-0321$ & HDS2936 & 2011.751 & 1 & - & - & - & - & & NR & & & \\
\hline $20334+4109$ & WSI67 & 2012.627 & 1 & 0.744 & 0.007 & $145.8^{*}$ & * 0.5 & & & & & \\
\hline $20335+2250$ & HDS2938 & 2011.573 & 1 & 1.553 & 0.010 & 119.2 & 0.3 & & & & & \\
\hline $20340+1244$ & TDT2378 & 2011.748 & 1 & - & - & - & - & & NR & & & \\
\hline $20341+4804$ & YSC116 & 2012.728 & 1 & 0.147 & 0.040 & 99.7 & 8.0 & & & & & \\
\hline $20341+4804$ & YSC116 & 2012.687 & 1 & 0.142 & 0.040 & 98.2 & 8.0 & & & & & \\
\hline $20351-0436$ & RST4671 & 2011.751 & 1 & 0.720 & 0.008 & $46.5^{*}$ & 0.3 & & & & & \\
\hline $20352+0019$ & RST5157 & 2011.756 & 1 & 1.734 & 0.009 & $293.4^{*}$ & ${ }^{*} 0.3$ & & & & & \\
\hline $20354+1121$ & YR16 & 2012.816 & 1 & 0.604 & 0.007 & $264.6^{*}$ & * 0.3 & & & & & \\
\hline $20355+3241$ & COU1632 & 2011.576 & 1 & 0.648 & 0.007 & $251.5^{*}$ & * 0.8 & & & & & \\
\hline $20357+3908$ & COU2289 & 2011.576 & 1 & 0.702 & 0.007 & $333.7^{*}$ & * 0.3 & & & & & \\
\hline $20359+3617$ & GII52Aa,Ab & 2012.726 & 1 & 0.354 & 0.007 & $216.2^{*}$ & * 0.8 & & ND & & & \\
\hline $20375+1050$ & HEI586 & 2012.816 & 1 & 0.561 & 0.008 & $219.9^{*}$ & * 0.5 & & & & & \\
\hline $20375+1436$ & BU151 & 2011.510 & 1 & 0.230 & 0.010 & $37.4^{*}$ & 1.6 & & & Mut2010e & -0.03 & -5.0 \\
\hline $20376+1835$ & COU224 & 2011.748 & 1 & 0.429 & 0.010 & $323.1^{*}$ & * 0.7 & & & & & \\
\hline $20380+1011$ & HDS2945 & 2011.748 & 1 & 0.356 & 0.007 & $77.8^{*}$ & 1.8 & & & & & \\
\hline $20381+2050$ & COU328 & 2011.576 & 1 & 1.029 & 0.007 & $155.9^{*}$ & * 0.3 & & & & & \\
\hline $20385+2945$ & COU1172 & 2011.726 & 1 & 0.325 & 0.007 & $278.5^{*}$ & * 0.4 & & & & & \\
\hline $20390+3702$ & COU2219 & 2011.576 & 1 & 0.911 & 0.007 & 42.8 & 0.3 & & & & & \\
\hline $20393+1529$ & HEI77 & 2011.740 & 1 & 1.382 & 0.011 & $40.9^{*}$ & 0.5 & & & & & \\
\hline $20393+2714$ & TDT2454 & 2011.723 & 1 & 0.934 & 0.007 & $45.4^{*}$ & 0.3 & & & & & \\
\hline $20394+2554$ & TDT2455 & 2011.759 & 1 & - & - & - & - & & NR & & & \\
\hline
\end{tabular}




\begin{tabular}{|c|c|c|c|c|c|c|c|c|c|c|c|c|}
\hline WDS & Name & Epoch & Bin. & $\begin{array}{c}\rho \\
(")\end{array}$ & $\begin{array}{l}\sigma_{\rho} \\
(")\end{array}$ & $\begin{array}{c}\theta \\
\left({ }^{\circ}\right)\end{array}$ & $\begin{array}{l}\sigma_{\theta} \\
\left(^{\circ}\right)\end{array}$ & $\Delta m$ & Notes & Orbit & $\begin{array}{r}\Delta \rho(\mathrm{O}-\mathrm{C}) \\
(")\end{array}$ & $\begin{array}{r}\Delta \theta(\mathrm{O}-\mathrm{C}) \\
\left({ }^{\circ}\right)\end{array}$ \\
\hline $20394+4318$ & COU2539 & 2012.726 & 1 & 0.353 & 0.007 & $64.0^{*}$ & 1.1 & & & & & \\
\hline $20396+0458$ & KUI99 & 2011.745 & 1 & 0.739 & 0.028 & $311.4^{*}$ & * 2.3 & & & Tok2017a & -0.04 & 3.7 \\
\hline $20396+0458$ & KUI99 & 2011.754 & 1 & 0.751 & 0.027 & $311.5^{*}$ & $* 2.3$ & & & Tok2017a & -0.03 & 3.8 \\
\hline $20396+0458$ & KUI99 & 2012.816 & 1 & 0.705 & 0.027 & $308.0^{*}$ & * 2.4 & & & Tok2017a & -0.04 & -0.3 \\
\hline $20397+3658$ & A1432 & 2011.576 & 1 & 0.430 & 0.007 & $115.7^{*}$ & * 0.5 & & & & & \\
\hline $20406+2948$ & HO137 & 2011.740 & 1 & 0.735 & 0.007 & $355.8^{*}$ & * 0.3 & & & & & \\
\hline $20406+2948$ & HO137 & 2011.830 & 1 & 0.754 & 0.007 & $355.7^{*}$ & * 0.3 & & & & & \\
\hline $20410+1206$ & HEI587 & 2011.754 & 1 & 1.218 & 0.007 & $35.8^{*}$ & 0.3 & & & & & \\
\hline $20411+3516$ & COU1963AB & 2011.742 & 1 & 0.242 & 0.007 & $183.4^{*}$ & * 0.4 & 0.20 & & & & \\
\hline $20411+3516$ & COU1963AB,C & 2011.742 & 1 & 1.751 & 0.018 & $43.9^{*}$ & 0.3 & & & & & \\
\hline $20412+2023$ & COU423 & 2011.723 & 1 & 0.430 & 0.007 & $160.4^{*}$ & * 1.6 & & & & & \\
\hline $20412+3001$ & COU1173 & 2011.723 & 1 & 1.159 & 0.007 & 48.3 & 0.3 & & & & & \\
\hline $20416+3000$ & COU1174 & 2011.573 & 1 & 0.341 & 0.007 & $30.8^{*}$ & 0.8 & & & & & \\
\hline $20416+3000$ & COU1174 & 2012.764 & 1 & 0.333 & 0.007 & $28.7^{*}$ & 0.3 & & & & & \\
\hline $20416+3950$ & COU2290 & 2012.687 & 1 & 0.548 & 0.007 & $39.4^{*}$ & 0.9 & & & & & \\
\hline $20418+1404$ & $\mathrm{~J} 2575$ & 2011.754 & 1 & - & - & - & - & & NR & & & \\
\hline $20419-0458$ & RST4676 & 2011.756 & 1 & 0.342 & 0.007 & $188.0^{*}$ & * 0.9 & & & & & \\
\hline $20423+1921$ & SHN39 & 2012.764 & 1 & 0.458 & 0.007 & 54.3 & 1.9 & & & & & \\
\hline $20424+3455$ & COU1965 & 2011.726 & 1 & 0.358 & 0.007 & $274.6^{*}$ & * 0.9 & & & & & \\
\hline $20432+3350$ & HDS2949 & 2011.759 & 1 & 0.929 & 0.007 & $164.5^{*}$ & * 0.4 & & & & & \\
\hline $20432+3350$ & HDS2949 & 2011.759 & 1 & 0.925 & 0.007 & 164.4 & 0.3 & & & & & \\
\hline $20432+3350$ & HDS2949 & 2012.687 & 1 & 0.951 & 0.007 & $165.5^{*}$ & * 0.5 & & & & & \\
\hline $20439-0557$ & RST4679 & 2011.756 & 1 & 0.337 & 0.011 & $356.0^{*}$ & * 1.2 & & & & & \\
\hline $20440+3839$ & COU2292 & 2012.687 & 1 & 0.303 & 0.007 & $238.1^{*}$ & * 0.4 & & & & & \\
\hline $20440+3839$ & COU2292 & 2011.759 & 1 & 0.296 & 0.007 & $235.9^{*}$ & * 0.9 & & & & & \\
\hline $20444+4103$ & COU2423 & 2011.726 & 1 & 0.283 & 0.007 & $183.2^{*}$ & * 0.5 & & & & & \\
\hline $20450+1244$ & BU64 & 2011.745 & 1 & 0.661 & 0.007 & $173.1^{*}$ & * 0.3 & & & USN2007a & 0.01 & $-1.1^{Q}$ \\
\hline $20455-0716$ & A3000 & 2011.756 & 1 & 0.672 & 0.007 & $63.7^{*}$ & 0.3 & & & & & \\
\hline $20456+1714$ & COU 425 & 2011.827 & 1 & 0.655 & 0.013 & 146.3 & 1.1 & & & & & \\
\hline $20459+0607$ & TDT2523 & 2012.816 & 1 & 1.650 & 0.008 & $80.2^{*}$ & 0.3 & & & & & \\
\hline $20464+3511$ & COU1810 & 2011.830 & 1 & 0.207 & 0.007 & 178.4 & 1.7 & & & & & \\
\hline $20464+3511$ & COU1810 & 2011.740 & 1 & 0.233 & 0.012 & 181.8 & 1.0 & & & & & \\
\hline $20466+1915$ & COU426 & 2011.573 & 1 & 0.247 & 0.007 & $159.2^{*}$ & * 2.2 & & & & & \\
\hline $20477+3258$ & COU1634 & 2011.830 & 1 & 0.184 & 0.040 & $133.1^{*}$ & * 8.0 & & & & & \\
\hline $20480+2704$ & COU826 & 2012.687 & 1 & 1.278 & 0.007 & $122.7^{*}$ & * 0.3 & & & & & \\
\hline $20481-0302$ & A174 & 2011.751 & 1 & 0.637 & 0.012 & $356.9^{*}$ & * 0.3 & & & & & \\
\hline $20482-0648$ & RST4681 & 2011.751 & 1 & 0.312 & 0.007 & 62.7 & 1.2 & & & & & \\
\hline $20485+0143$ & RST5471 & 2011.756 & 1 & 0.657 & 0.007 & $17.8^{*}$ & 0.3 & & & & & \\
\hline $20489-0214$ & HO142 & 2012.816 & 1 & 1.004 & 0.007 & $9.6^{*}$ & 0.4 & & & & & \\
\hline $20490+2540$ & HDS2966 & 2011.827 & 1 & 0.555 & 0.007 & $222.2^{*}$ & * 1.0 & & & & & \\
\hline $20492-0302$ & RST4682 & 2011.756 & 1 & 0.930 & 0.007 & $1.7^{*}$ & 0.3 & & & & & \\
\hline $20492+0502$ & HDS2967 & 2011.754 & 1 & 0.399 & 0.010 & $189.9^{*}$ & $* 2.4$ & & & & & \\
\hline $20492+4525$ & COU2646 & 2012.764 & 1 & 0.383 & 0.007 & $265.1^{*}$ & * 0.4 & & & & & \\
\hline $20494+1124$ & J194 & 2012.816 & 1 & 0.600 & 0.007 & $320.5^{*}$ & * 0.3 & & & Sod1999 & 0.02 & 2.5 \\
\hline $20495+4035$ & COU2426 & 2011.576 & 1 & 1.237 & 0.007 & $328.5^{*}$ & * 0.3 & & & & & \\
\hline $20496+5047$ & HDS2968 & 2011.759 & 1 & 0.331 & 0.007 & 69.4 & 0.8 & & & & & \\
\hline $20499-0131$ & RST4684 & 2011.754 & 1 & 0.271 & 0.007 & 2.6 & 0.3 & & & & & \\
\hline $20508+2232$ & HDS2972 & 2011.745 & 1 & - & - & - & - & & NR & & & \\
\hline $20514-0538$ & STF2729 & 2011.756 & 1 & 0.832 & 0.007 & $27.4^{*}$ & 0.3 & & & Mdz2017 & 0.00 & 0.7 \\
\hline $20514-0538$ & STF 2729 & 2012.816 & 1 & 0.815 & 0.007 & $28.4^{*}$ & 0.3 & & & Mdz2017 & -0.00 & 1.0 \\
\hline $20527+4607$ & A750 & 2011.573 & 1 & - & - & - & - & & NR & & & \\
\hline $20528+3132$ & TDT2599 & 2012.777 & 2 & 1.714 & 0.015 & $144.5^{*}$ & * 0.4 & & & & & \\
\hline $20528+3646$ & COU2221 & 2012.682 & 1 & 0.318 & 0.007 & $202.6^{*}$ & * 1.7 & & & & & \\
\hline $20530+0427$ & TDT2603 & 2011.748 & 1 & - & - & - & - & & NR & & & \\
\hline
\end{tabular}




\begin{tabular}{|c|c|c|c|c|c|c|c|c|c|c|c|c|}
\hline WDS & Name & Epoch & Bin. & $\begin{array}{c}\rho \\
(")\end{array}$ & $\begin{array}{l}\sigma_{\rho} \\
(")\end{array}$ & $\begin{array}{c}\theta \\
\left({ }^{\circ}\right)\end{array}$ & $\begin{array}{l}\sigma_{\theta} \\
\left(^{\circ}\right)\end{array}$ & $\Delta m$ & Notes & Orbit & $\begin{array}{r}\Delta \rho(\mathrm{O}-\mathrm{C}) \\
(")\end{array}$ & $\begin{array}{r}\Delta \theta(\mathrm{O}-\mathrm{C}) \\
\left({ }^{\circ}\right)\end{array}$ \\
\hline $20535+0300$ & HEI819 & 2011.745 & 1 & 0.210 & 0.007 & $135.0^{*}$ & * 2.0 & & & & & \\
\hline $20535+2630$ & COU1177 & 2011.726 & 1 & 0.244 & 0.007 & $13.9^{*}$ & 1.0 & & & & & \\
\hline $20539-0323$ & RST4686 & 2011.754 & 1 & 0.441 & 0.015 & $103.6^{*}$ & * 1.2 & & & & & \\
\hline $20549+3709$ & COU2295 & 2011.576 & 1 & 0.436 & 0.007 & $182.6^{*}$ & ${ }^{*} 0.3$ & & & & & \\
\hline $20549+3803$ & SEI1309AB & 2012.682 & 1 & 6.421 & 0.032 & 120.7 & 0.3 & 2.29 & & & & \\
\hline $20550+2805$ & BU367 & 2012.685 & 1 & 0.245 & 0.010 & $153.5^{*}$ & * 1.1 & & & Sca2003e & 0.08 & -25.5 \\
\hline $20550+2805$ & BU367 & 2012.685 & 1 & 0.220 & 0.007 & 153.4 & 2.6 & & & Sca2003e & 0.05 & -25.6 \\
\hline $20554+0836$ & J1318 & 2011.745 & 1 & 0.873 & 0.007 & $138.9^{*}$ & ${ }^{*} 0.3$ & & & & & \\
\hline $20556+3809$ & COU2222 & 2012.682 & 1 & 0.357 & 0.007 & $110.9^{*}$ & * 1.1 & & & & & \\
\hline $20556+3809$ & COU2222 & 2011.759 & 1 & 0.393 & 0.007 & $288.0^{*}$ & * 0.8 & & & & & \\
\hline $20558+2131$ & COU727 & 2011.726 & 1 & 1.104 & 0.009 & $353.4^{*}$ & ${ }^{*} 0.3$ & & & & & \\
\hline $20560+3546$ & COU2134 & 2011.740 & 1 & 0.539 & 0.007 & $261.1^{*}$ & ${ }^{*} 0.3$ & & & & & \\
\hline $20564+4308$ & COU2544 & 2012.764 & 1 & 0.317 & 0.008 & $161.2^{*}$ & * 0.3 & & & & & \\
\hline $20565+2118$ & A 2797 & 2012.764 & 1 & 0.135 & 0.040 & $237.2^{*}$ & ${ }^{*} 8.0$ & & & & & \\
\hline $20565+2118$ & A 2797 & 2012.726 & 1 & 0.159 & 0.040 & 232.0 & 8.0 & & & & & \\
\hline $20566+3858$ & A1436 & 2012.726 & 1 & 0.514 & 0.008 & $50.0^{*}$ & 0.6 & & & & & \\
\hline $20568+3348$ & COU1812 & 2011.576 & 1 & 1.110 & 0.010 & $104.4^{*}$ & ${ }^{*} 0.3$ & & & & & \\
\hline $20569+1059$ & HDS2985 & 2011.745 & 1 & 0.630 & 0.007 & $292.5^{*}$ & * 0.9 & & & & & \\
\hline $20577+3848$ & ES2056 & 2012.777 & 2 & 2.658 & 0.019 & $74.8^{*}$ & 0.3 & & & & & \\
\hline $20581+4043$ & COU2430AB & 2012.685 & 1 & 0.413 & 0.007 & $252.9^{*}$ & ${ }^{*} 1.4$ & & & & & \\
\hline $20581+4043$ & COU2430A,BC & 2012.685 & 1 & 3.047 & 0.015 & 175.5 & 0.3 & & & & & \\
\hline $20581+4043$ & COU2430A,BC & 2012.685 & 1 & 3.004 & 0.015 & 176.2 & 0.3 & 4.96 & & & & \\
\hline $20582+1038$ & $\mathrm{~A} 1215$ & 2012.816 & 1 & 0.431 & 0.007 & $209.3^{*}$ & ${ }^{*} 0.3$ & & & & & \\
\hline $20584+0118$ & RST5474 & 2012.816 & 1 & 1.993 & 0.010 & $193.5^{*}$ & ${ }^{*} 0.3$ & & & & & \\
\hline $20584+3344$ & COU1635 & 2011.827 & 1 & 1.587 & 0.008 & 51.6 & 0.5 & & & & & \\
\hline $20588-0402$ & RST4687 & 2011.751 & 1 & - & - & - & - & & $\mathrm{NR}$ & & & \\
\hline $20590+3218$ & COU1636 & 2011.742 & 1 & 0.815 & 0.007 & $189.7^{*}$ & ${ }^{*} 0.8$ & 0.30 & & & & \\
\hline $20590+3218$ & COU1636 & 2011.827 & 1 & 0.831 & 0.007 & $189.1^{*}$ & ${ }^{*} 0.3$ & 0.40 & & & & \\
\hline $20591+0418$ & STF2737 & 2011.754 & 1 & 0.465 & 0.007 & $285.6^{*}$ & ${ }^{*} 0.6$ & & & Tok2017c & -0.01 & 1.9 \\
\hline $20592+3314$ & COU1637 & 2011.726 & 1 & 0.121 & 0.048 & $3.7^{*}$ & 7.1 & & & & & \\
\hline $20599+4016$ & HDS2989Da,Db & 2012.764 & 1 & 0.205 & 0.015 & 144.6 & 8.3 & & & Tok2019e & 0.02 & -1.6 \\
\hline $21000-0436$ & RST4688 & 2011.754 & 1 & 0.293 & 0.008 & $291.5^{*}$ & ${ }^{*} 1.4$ & & & & & \\
\hline $21000+3740$ & COU2297 & 2011.742 & 1 & 0.268 & 0.007 & $233.8^{*}$ & ${ }^{*} 0.3$ & 0.40 & & & & \\
\hline $21000+3740$ & COU2297 & 2011.830 & 1 & 0.282 & 0.007 & $235.8^{*}$ & * 0.3 & & & & & \\
\hline $21000+3740$ & COU2297 & 2012.764 & 1 & 0.311 & 0.010 & $238.1^{*}$ & * 1.5 & & & & & \\
\hline $21000+4004$ & KUI103 & 2011.573 & 1 & 0.582 & 0.007 & $27.3^{*}$ & 0.3 & & & Doc $2016 b$ & -0.02 & 0.5 \\
\hline $21000+4004$ & KUI103 & 2011.726 & 1 & 0.598 & 0.007 & $28.7^{*}$ & 0.3 & & & Doc2016b & -0.01 & 0.3 \\
\hline $21000+4004$ & KUI103 & 2012.627 & 1 & 0.646 & 0.010 & $35.1^{*}$ & 0.3 & & & Doc2016b & -0.02 & -1.7 \\
\hline $21000+4004$ & KUI103 & 2012.628 & 1 & 0.633 & 0.012 & $39.8^{*}$ & 0.3 & & & Doc2016b & -0.04 & 3.0 \\
\hline $21001+0731$ & KUI102 & 2012.816 & 1 & 0.135 & 0.040 & 287.4 & 8.0 & & & Hrt2014b & -0.01 & -0.6 \\
\hline $21004+3022$ & COU1179 & 2012.854 & 1 & 0.895 & 0.007 & $358.9^{*}$ & ${ }^{*} 0.3$ & & & & & \\
\hline $21004+3022$ & COU1179 & 2012.854 & 1 & 0.877 & 0.014 & $359.3^{*}$ & ${ }^{*} 0.3$ & & & & & \\
\hline $21004+3411$ & BU1329 & 2012.854 & 1 & 0.305 & 0.007 & $217.3^{*}$ & ${ }^{*} 0.3$ & & & & & \\
\hline $21004+3411$ & BU1329 & 2012.854 & 1 & 0.321 & 0.007 & $218.7^{*}$ & * 0.3 & & & & & \\
\hline $21008+2057$ & A176 & 2012.854 & 1 & 0.249 & 0.007 & $175.2^{*}$ & ${ }^{*} 0.6$ & & & & & \\
\hline $21010+1910$ & COU523 & 2011.745 & 1 & - & - & - & - & & NR & & & \\
\hline $21019+2340$ & COU128 & 2012.726 & 1 & 0.200 & 0.007 & $146.2^{*}$ & * 1.4 & & & & & \\
\hline $21021+1423$ & A1687 & 2011.745 & 1 & 0.790 & 0.007 & $189.0^{*}$ & ${ }^{*} 0.3$ & & & & & \\
\hline $21023+2037$ & HDS2998 & 2011.830 & 1 & 0.104 & 0.043 & 130.4 & 6.7 & & & & & \\
\hline $21026+2141$ & BU69 & 2011.808 & 1 & 0.323 & 0.007 & $359.7^{*}$ & ${ }^{*} 0.6$ & & & Zir2014a & -0.07 & -0.4 \\
\hline $21031+0132$ & STF 2744 & 2011.748 & 1 & 1.241 & 0.007 & $112.2^{*}$ & * 0.3 & & & Izm2019 & -0.02 & -0.0 \\
\hline
\end{tabular}




\begin{tabular}{|c|c|c|c|c|c|c|c|c|c|c|c|c|}
\hline WDS & Name & Epoch & Bin. & $\stackrel{\rho}{\rho}$ & $\begin{array}{l}\sigma_{\rho} \\
(")\end{array}$ & $\begin{array}{c}\theta \\
\left({ }^{\circ}\right)\end{array}$ & $\begin{array}{l}\sigma_{\theta} \\
\left(^{\circ}\right)\end{array}$ & $\Delta m$ & Notes & Orbit & $\begin{array}{r}\Delta \rho(\mathrm{O}-\mathrm{C}) \\
(")\end{array}$ & $\begin{array}{r}\Delta \theta(\mathrm{O}-\mathrm{C}) \\
\left({ }^{\circ}\right)\end{array}$ \\
\hline $21032-0610$ & RST4689 & 2011.754 & 1 & 0.844 & 0.007 & $83.5^{*}$ & 0.4 & & & & & \\
\hline $21037+4652$ & YSC14 & 2012.755 & 1 & - & - & - & - & & NR & & & \\
\hline $21040+4225$ & COU2433 & 2012.755 & 1 & 0.347 & 0.007 & $230.8^{*}$ & 0.7 & & & & & \\
\hline $21047+0332$ & SE3AB & 2011.745 & 1 & - & - & - & - & & NR & & & \\
\hline $21047+0332$ & SE3BC & 2011.748 & 1 & 0.602 & 0.007 & 129.6 & 0.3 & 0.13 & & & & \\
\hline $21047+0332$ & STF2749A,BC & 2011.748 & 1 & 3.065 & 0.015 & $181.4^{*}$ & 0.3 & & & & & \\
\hline $21050-0403$ & RST4690 & 2011.756 & 1 & 0.885 & 0.007 & $7.3^{*}$ & 0.3 & & & & & \\
\hline $21051+0757$ & HDS3004 & 2011.754 & 1 & - & - & - & - & & NR & & & \\
\hline $21051+0757$ & HDS3004 & 2012.816 & 1 & 0.180 & 0.007 & 64.0 & 1.8 & & & Tok2015c & -0.02 & 1.0 \\
\hline $21052+1936$ & HDS3005 & 2012.764 & 1 & 0.340 & 0.007 & 3.0 & 0.5 & & & & & \\
\hline $21053+0044$ & HDS3007 & 2011.751 & 1 & - & - & - & - & & NR & & & \\
\hline $21053+2807$ & HDS3006 & 2012.685 & 1 & 0.693 & 0.008 & $11.2^{*}$ & 0.3 & & & & & \\
\hline $21053+2807$ & HDS3006 & 2012.685 & 1 & 0.689 & 0.021 & 11.4 & 1.0 & & & & & \\
\hline $21054-0744$ & RST4691 & 2011.756 & 1 & 0.479 & 0.027 & 70.7 & 1.5 & & & & & \\
\hline $21054+2824$ & MLB666 & 2012.848 & 1 & 1.351 & 0.021 & 175.9 & 1.0 & & & & & \\
\hline $21054+2824$ & MLB666 & 2012.848 & 1 & 1.351 & 0.021 & 175.9 & 1.0 & & & & & \\
\hline $21055+2434$ & COU129 & 2012.854 & 1 & 0.701 & 0.007 & $75.7^{*}$ & 0.8 & & & & & \\
\hline $21056+1443$ & HEI78 & 2011.748 & 1 & 1.844 & 0.009 & 54.0 & 0.3 & & & & & \\
\hline $21057+2508$ & COU130 & 2011.726 & 1 & 1.323 & 0.007 & $150.4^{*}$ & 0.3 & & & & & \\
\hline $21058+1939$ & $\mathrm{~J} 2702$ & 2012.764 & 1 & 5.386 & 0.027 & $178.5^{*}$ & 0.3 & 0.23 & & & & \\
\hline $21059+0152$ & YSC71 & 2012.816 & 1 & - & - & - & - & & NR & & & \\
\hline $21059+2118$ & A178 & 2012.848 & 1 & 1.046 & 0.007 & $64.2^{*}$ & 0.3 & & & & & \\
\hline $21065+2655$ & COU527AB & 2012.755 & 1 & 0.309 & 0.007 & $131.1^{*}$ & 0.9 & & & & & \\
\hline $21065+2655$ & COU527AC & 2012.755 & 1 & 5.894 & 0.029 & $49.6^{*}$ & 0.3 & 2.98 & & & & \\
\hline $21068+2306$ & HU364 & 2011.759 & 1 & 0.241 & 0.008 & $111.0^{*}$ & 1.3 & & & & & \\
\hline $21072+2833$ & COU528 & 2011.740 & 1 & 1.204 & 0.007 & $69.8^{*}$ & 0.4 & & & & & \\
\hline $21072+2833$ & COU528 & 2011.827 & 1 & 1.207 & 0.013 & $70.1^{*}$ & 0.3 & & & & & \\
\hline $21074-0810$ & HDS3010 & 2011.754 & 1 & 0.852 & 0.007 & $30.0^{*}$ & 0.3 & & & & & \\
\hline $21074+3444$ & COU1814 & 2011.740 & 1 & 0.331 & 0.014 & $201.0^{*}$ & 1.6 & & & & & \\
\hline $21077+3255$ & COU1330 & 2012.854 & 1 & 0.393 & 0.007 & $218.7^{*}$ & 0.5 & & & & & \\
\hline $21080+0509$ & STT527 & 2011.751 & 1 & 0.345 & 0.007 & $115.0^{*}$ & 0.3 & & & Pop1995a & -0.03 & 5.9 \\
\hline $21083+2913$ & COU1331 & 2011.742 & 1 & 0.311 & 0.007 & $31.5^{*}$ & 0.4 & 0.60 & & & & \\
\hline $21091+2922$ & COU1332 & 2011.759 & 1 & 0.210 & 0.011 & $199.4^{*}$ & 0.9 & & & & & \\
\hline $21091+2922$ & COU1332 & 2012.685 & 1 & 0.253 & 0.007 & 202.6 & 3.3 & & & & & \\
\hline $21091+2922$ & COU1332 & 2012.685 & 1 & 0.229 & 0.015 & $200.5^{*}$ & 1.1 & & & & & \\
\hline $21092+3620$ & COU1815 & 2011.726 & 1 & 0.359 & 0.007 & $99.5^{*}$ & 0.8 & & & & & \\
\hline $21092+3638$ & COU1966 & 2012.756 & 1 & 1.332 & 0.007 & $311.2^{*}$ & 0.3 & & & & & \\
\hline $21096-0940$ & RST4075 & 2011.754 & 1 & 1.624 & 0.014 & 43.6 & 0.3 & & & & & \\
\hline $21097+1434$ & OL132 & 2011.917 & 1 & - & - & - & - & & NR & & & \\
\hline $21106+1650$ & HU367 & 2012.854 & 1 & 0.302 & 0.007 & $342.1^{*}$ & 1.0 & & & & & \\
\hline $21107+1334$ & HEI186 & 2012.816 & 1 & - & - & - & - & & NR & & & \\
\hline $21109+1801$ & COU69 & 2012.848 & 1 & 1.505 & 0.334 & 150.8 & 8.2 & & & & & \\
\hline $21109+1801$ & COU69 & 2012.848 & 1 & 1.505 & 0.334 & 150.8 & 8.2 & & & & & \\
\hline $21109+2925$ & BAG29 & 2011.827 & 1 & 0.215 & 0.016 & 106.3 & 1.9 & & & Bag2010 & -0.04 & -7.6 \\
\hline $21109+2925$ & BAG29 & 2012.726 & 1 & 0.325 & 0.010 & $123.1^{*}$ & 0.3 & & & Bag2010 & 0.06 & -0.8 \\
\hline $21110+0102$ & $\mathrm{~A} 2287$ & 2011.754 & 1 & 0.108 & 0.049 & 11.8 & 7.0 & & & & & \\
\hline $21110+3320$ & VKI41 & 2012.685 & 1 & 1.192 & 0.007 & $309.4^{*}$ & 0.4 & & & & & \\
\hline $21115+2144$ & COU227 & 2011.827 & 1 & 0.512 & 0.007 & $115.8^{*}$ & 0.8 & & & FMR2012b & -0.01 & 1.3 \\
\hline
\end{tabular}




\begin{tabular}{|c|c|c|c|c|c|c|c|c|c|c|c|c|}
\hline WDS & Name & Epoch & Bin. & $\begin{array}{c}\rho \\
(")\end{array}$ & $\begin{array}{l}\sigma_{\rho} \\
(")\end{array}$ & $\begin{array}{l}\theta \\
\left({ }^{\circ}\right)\end{array}$ & $\begin{array}{l}\sigma_{\theta} \\
\left({ }^{\circ}\right)\end{array}$ & $\Delta m$ & Notes & Orbit & $\begin{array}{r}\Delta \rho(\mathrm{O}-\mathrm{C}) \\
\text { (") }\end{array}$ & $\begin{array}{r}\Delta \theta(\mathrm{O}-\mathrm{C}) \\
\left({ }^{\circ}\right)\end{array}$ \\
\hline $21115+2144$ & COU227 & 2012.854 & 1 & 0.511 & 0.007 & $116.3^{*}$ & * 0.5 & & & FMR2012b & -0.01 & -0.0 \\
\hline $21119+2524$ & COU643 & 2011.726 & 1 & 0.173 & 0.040 & $130.0^{*}$ & $* 8.0$ & & & & & \\
\hline $21122-0203$ & A179 & 2011.756 & 1 & 0.283 & 0.010 & $180.3^{*}$ & $* 0.7$ & & & & & \\
\hline $21125+2821$ & HO152 & 2011.726 & 1 & 0.181 & 0.040 & 140.7 & 8.0 & & & Doc $2016 \mathrm{~g}$ & -0.01 & 1.6 \\
\hline $21125+2821$ & HO152 & 2012.756 & 1 & 0.192 & 0.040 & $139.5^{*}$ & $* 8.0$ & & & Doc2016g & 0.01 & -1.9 \\
\hline $21133+4655$ & A884 & 2011.759 & 1 & 0.435 & 0.007 & 114.4 & 0.6 & & & & & \\
\hline $21135+1559$ & HU767 & 2011.748 & 1 & 0.086 & 0.040 & 165.3 & 8.0 & & & Hrt1996a & 0.05 & 89.8 \\
\hline $21135+1559$ & HU767 & 2011.827 & 1 & 0.114 & 0.040 & 170.6 & 8.0 & & & Hrt1996a & 0.08 & 95.0 \\
\hline $21138+3711$ & COU1816AB & 2012.726 & 1 & 1.844 & 0.009 & $247.7^{*}$ & $* 0.3$ & & & & & \\
\hline $21138+3711$ & COU1816AB & 2012.726 & 2 & 1.849 & 0.015 & $247.8^{*}$ & $* 0.3$ & & & & & \\
\hline $21138+3711$ & COU1816AC & 2012.726 & 2 & 10.038 & 0.050 & $336.1^{*}$ & * 0.3 & & ND & & & \\
\hline $21145+1000$ & STT535AB & 2011.748 & 1 & 0.300 & 0.007 & $24.3^{*}$ & 1.4 & & & Mut2008 & -0.04 & $-3.4^{Q}$ \\
\hline $21151+4227$ & COU2228 & 2011.759 & 1 & 0.502 & 0.007 & $28.5^{*}$ & 0.5 & 1.00 & & & & \\
\hline $21159+2858$ & HDS3025 & 2012.854 & 1 & 0.221 & 0.010 & 45.9 & 2.8 & & & & & \\
\hline $21161+3747$ & COU1819 & 2012.756 & 1 & 0.696 & 0.010 & $328.8^{*}$ & ${ }^{*} 0.4$ & & & & & \\
\hline $21166-0037$ & HDS3029 & 2011.756 & 1 & 0.640 & 0.007 & 118.0 & 0.7 & & & & & \\
\hline $21166+4009$ & COU1972 & 2011.759 & 1 & - & - & - & - & & NR & & & \\
\hline $21169+4009$ & COU1972 & 2012.756 & 1 & 0.157 & 0.040 & 145.2 & 8.0 & & & & & \\
\hline $21170+4704$ & COU2437 & 2012.726 & 1 & 0.430 & 0.007 & $235.3^{*}$ & ${ }^{*} 0.6$ & & & & & \\
\hline $21171+3546$ & BU162 & 2012.777 & 1 & 1.228 & 0.007 & $252.7^{*}$ & $* 0.3$ & & & & & \\
\hline $21179+1831$ & HU368 & 2012.777 & 1 & 0.249 & 0.007 & 131.8 & 1.7 & & & & & \\
\hline $21180+3049$ & COU1183 & 2012.777 & 1 & 0.253 & 0.013 & $195.9^{*}$ & * 1.0 & & & & & \\
\hline $21183+4140$ & COU2302 & 2012.777 & 1 & 1.276 & 0.007 & $60.9^{*}$ & 0.3 & & & & & \\
\hline $21187+2118$ & HDS3033Aa,Ab & 2012.854 & 1 & - & - & - & - & & NR & & & \\
\hline $21187+3348$ & HU770 & 2012.848 & 1 & 0.894 & 0.014 & 133.4 & 0.8 & & & & & \\
\hline $21187+3348$ & HU770 & 2012.848 & 1 & 0.894 & 0.014 & 133.4 & 0.8 & & & & & \\
\hline $21206+1310$ & HDS3038 & 2011.748 & 1 & - & - & - & - & & NR & & & \\
\hline $21209+0307$ & BU838 & 2011.748 & 1 & 1.628 & 0.008 & $154.6^{*}$ & ${ }^{*} 0.3$ & & & Zir2015a & -0.02 & 1.4 \\
\hline $21209+0307$ & BU838 & 2011.756 & 1 & 1.639 & 0.008 & $154.9^{*}$ & ${ }^{*} 0.3$ & & & Zir2015a & -0.01 & 1.8 \\
\hline $21209+2720$ & COU729 & 2012.854 & 1 & 0.213 & 0.008 & $138.1^{*}$ & * 0.3 & & & & & \\
\hline $21212+4003$ & COU2230 & 2011.726 & 1 & 0.440 & 0.007 & $84.0^{*}$ & 0.4 & & & & & \\
\hline $21214+0253$ & STT435 & 2011.748 & 1 & 0.684 & 0.007 & 57.9 & 0.3 & & & $\mathrm{PkO} 2018 \mathrm{~b}$ & 0.00 & -0.8 \\
\hline $21224+2927$ & COU1041 & 2011.740 & 1 & 1.246 & 0.008 & 90.5 & 0.3 & & & & & \\
\hline $21240+2445$ & COU231 & 2011.830 & 1 & 0.461 & 0.007 & $125.2^{*}$ & ${ }^{*} 0.6$ & & & & & \\
\hline $21243+0343$ & A2288 & 2011.748 & 1 & 0.364 & 0.013 & $227.4^{*}$ & * 1.0 & & & Tok2015c & -0.01 & -3.1 \\
\hline $21248+4106$ & COU2305 & 2011.726 & 1 & 0.350 & 0.007 & $102.1^{*}$ & ${ }^{*} 0.3$ & & & & & \\
\hline $21252+1828$ & COU430 & 2011.751 & 1 & 0.582 & 0.007 & $233.8^{*}$ & ${ }^{*} 0.3$ & & & & & \\
\hline $21252+2600$ & TDT2952 & 2012.854 & 1 & 0.970 & 0.007 & $119.2^{*}$ & * 0.6 & & & & & \\
\hline $21253+2928$ & COU940 & 2012.685 & 1 & 0.357 & 0.007 & $269.0^{*}$ & * 1.1 & & & & & \\
\hline $21254+1121$ & A 887 & 2011.748 & 1 & 0.183 & 0.040 & $180.2^{*}$ & * 8.0 & & & & & \\
\hline $21256+4138$ & A618 & 2012.726 & 1 & 0.556 & 0.009 & $276.5^{*}$ & * 0.3 & & & & & \\
\hline $21257+4043$ & COU2306 & 2012.867 & 1 & 0.579 & 0.007 & $20.4^{*}$ & 0.6 & & & & & \\
\hline $21257+4043$ & COU2306 & 2011.759 & 1 & 0.584 & 0.007 & $21.1^{*}$ & 0.5 & & & & & \\
\hline $21260+2422$ & COU534 & 2011.726 & 1 & 0.337 & 0.008 & $110.7^{*}$ & * 0.5 & & & & & \\
\hline $21261-0010$ & RST5164 & 2011.756 & 1 & 0.856 & 0.007 & $307.4^{*}$ & * 0.8 & & & & & \\
\hline $21264+3909$ & A1442 & 2012.777 & 1 & 1.258 & 0.007 & $272.9^{*}$ & ${ }^{*} 0.3$ & & & & & \\
\hline $21268+3731$ & COU1821 & 2011.830 & 1 & 0.641 & 0.007 & $95.5^{*}$ & 0.3 & & & & & \\
\hline $21281+4110$ & COU2231 & 2012.868 & 1 & 0.179 & 0.040 & $28.7^{*}$ & 8.0 & & & & & \\
\hline $21287+0743$ & HU276 & 2011.751 & 1 & 0.892 & 0.007 & $28.0^{*}$ & 0.4 & & & & & \\
\hline $21287+1810$ & HU490AB & 2011.740 & 1 & 0.346 & 0.007 & $312.6^{*}$ & * 0.3 & & & & & \\
\hline $21287+1810$ & HU490AB & 2012.756 & 1 & 0.343 & 0.007 & $309.2^{*}$ & * 0.9 & & & & & \\
\hline $21296+4626$ & HDS3059 & 2012.685 & 1 & 0.669 & 0.007 & 142.0 & 0.6 & & & & & \\
\hline $21297+1719$ & COU535 & 2012.870 & 1 & 0.671 & 0.007 & $31.4^{*}$ & 0.3 & & & & & \\
\hline
\end{tabular}




\begin{tabular}{|c|c|c|c|c|c|c|c|c|c|c|c|c|}
\hline WDS & Name & Epoch & Bin. & $\begin{array}{c}\rho \\
(")\end{array}$ & $\begin{array}{l}\sigma_{\rho} \\
(")\end{array}$ & $\begin{array}{c}\theta \\
\left({ }^{\circ}\right)\end{array}$ & $\begin{array}{l}\sigma_{\theta} \\
\left(^{\circ}\right)\end{array}$ & $\Delta m$ & Notes & Orbit & $\begin{array}{r}\Delta \rho(\mathrm{O}-\mathrm{C}) \\
(")\end{array}$ & $\begin{array}{r}\Delta \theta(\mathrm{O}-\mathrm{C}) \\
\left({ }^{\circ}\right)\end{array}$ \\
\hline $21297+4732$ & A 767 & 2011.759 & 1 & 1.269 & 0.007 & $171.0^{*}$ & * 0.3 & & & & & \\
\hline $21301+3050$ & COU941 & 2011.830 & 1 & - & - & - & - & & NR & & & \\
\hline $21301+3050$ & COU941 & 2012.854 & 1 & - & - & - & - & & NR & & & \\
\hline $21303+4740$ & COU2440 & 2012.685 & 1 & 0.477 & 0.007 & $180.2^{*}$ & * 0.3 & & & & & \\
\hline $21303+4740$ & COU2440 & 2011.759 & 1 & 0.507 & 0.007 & $178.9^{*}$ & * 0.6 & & & & & \\
\hline $21304+3504$ & HLD45 & 2012.868 & 1 & 1.300 & 0.007 & $197.9^{*}$ & * 0.3 & & & & & \\
\hline $21307+0005$ & A888 & 2012.816 & 1 & 0.553 & 0.007 & $217.3^{*}$ & * 0.4 & & & & & \\
\hline $21307+2258$ & COU134 & 2011.830 & 1 & 1.432 & 0.054 & $3.6^{*}$ & 1.4 & & & & & \\
\hline $21308+3446$ & COU1479 & 2012.756 & 1 & 0.717 & 0.027 & $143.0^{*}$ & * 0.8 & & & & & \\
\hline $21308+3446$ & COU1479 & 2011.740 & 1 & 0.757 & 0.027 & $147.5^{*}$ & $*$ * 1.1 & & & & & \\
\hline $21321+4828$ & COU2442 & 2012.726 & 1 & 0.334 & 0.019 & 121.8 & 2.4 & & & & & \\
\hline $21322+0013$ & ALC4 & 2011.751 & 1 & 1.482 & 0.007 & 132.0 & 0.3 & & & & & \\
\hline $21324+2434$ & MCT12 & 2012.854 & 2 & 1.455 & 0.015 & $243.1^{*}$ & * 0.3 & & & & & \\
\hline $21328-0053$ & HDS3064 & 2011.751 & 1 & 0.294 & 0.007 & 177.7 & 0.4 & & & & & \\
\hline $21328-0053$ & HDS3064 & 2011.751 & 1 & 0.294 & 0.007 & 177.7 & 0.4 & & & & & \\
\hline $21328+0159$ & A2290 & 2011.756 & 1 & 0.499 & 0.007 & $260.4^{*}$ & * 0.4 & & & & & \\
\hline $21328+3904$ & A1443 & 2012.868 & 1 & 0.250 & 0.007 & $242.9^{*}$ & ${ }^{*} 1.6$ & & & & & \\
\hline $21340+3844$ & COU1976 & 2012.868 & 1 & 0.407 & 0.007 & $29.2^{*}$ & 0.4 & & & & & \\
\hline $21349+0308$ & A2291 & 2012.816 & 1 & 1.009 & 0.007 & $93.1^{*}$ & 0.3 & & & & & \\
\hline $21352+2124$ & BU74 & 2012.857 & 1 & 0.982 & 0.007 & $336.4^{*}$ & * 0.3 & & & & & \\
\hline $21352+2124$ & BU74 & 2012.857 & 1 & 0.987 & 0.007 & $337.0^{*}$ & * 0.3 & & & & & \\
\hline $21355+2427$ & HU371 & 2011.740 & 1 & 0.210 & 0.007 & $319.1^{*}$ & ${ }^{*} 2.3$ & & & WSI2006b & -0.06 & -0.9 \\
\hline $21365+4304$ & COU2233 & 2011.759 & 1 & 0.153 & 0.040 & 118.0 & 8.0 & & & & & \\
\hline $21368+3217$ & COU1184 & 2011.740 & 1 & 0.224 & 0.007 & 160.3 & 0.7 & & & & & \\
\hline $21372+0054$ & ARU22 & 2012.816 & 1 & - & - & - & - & & NR & & & \\
\hline $21376+0035$ & ARU4 & 2011.751 & 1 & 0.321 & 0.047 & 120.6 & 2.6 & & & & & \\
\hline $21379+2743$ & HDS3080 & 2011.748 & 1 & 3.317 & 0.017 & $18.9^{*}$ & 0.3 & & & & & \\
\hline $21379+2743$ & HDS3080 & 2012.685 & 1 & 3.286 & 0.016 & $18.6^{*}$ & 0.3 & & & & & \\
\hline $21379+2743$ & HDS3080 & 2012.685 & 1 & 3.235 & 0.016 & 19.3 & 0.3 & & & & & \\
\hline $21383+2336$ & HU372 & 2012.854 & 1 & 0.292 & 0.007 & $71.1^{*}$ & 0.3 & & & USN2002 & 0.06 & $-6.2^{Q}$ \\
\hline $21391+1252$ & ROE128 & 2012.889 & 1 & 1.353 & 0.008 & $262.5^{*}$ & * 0.3 & & & & & \\
\hline $21391+3356$ & WSI120 & 2012.764 & 1 & 1.410 & 0.007 & $54.8^{*}$ & 0.3 & & & & & \\
\hline $21393+2043$ & STT445 & 2012.870 & 1 & 1.017 & 0.007 & $120.2^{*}$ & * 0.3 & & & & & \\
\hline $21393+4625$ & COU2312 & 2011.759 & 1 & 0.320 & 0.007 & $161.7^{*}$ & * 0.3 & & & & & \\
\hline $21395+3551$ & COU1335 & 2011.830 & 1 & 0.548 & 0.009 & $288.4^{*}$ & * 0.5 & & & & & \\
\hline $21396+0431$ & HEI822 & 2011.756 & 1 & 1.206 & 0.007 & $0.1^{*}$ & 0.3 & & & & & \\
\hline $21399+1914$ & COU330 & 2011.830 & 1 & 0.507 & 0.007 & 13.3 & 0.3 & & & & & \\
\hline $21399+1914$ & COU330 & 2011.830 & 1 & 0.560 & 0.014 & 13.5 & 0.5 & & & & & \\
\hline $21399+2737$ & HDS3083AB & 2011.860 & 1 & - & - & - & - & & NR & & & \\
\hline $21399+2737$ & HDS3083AB & 2012.726 & 1 & - & - & - & - & & NR & & & \\
\hline $21399+2737$ & HDS3083AB & 2012.764 & 1 & - & - & - & - & & NR & & & \\
\hline $21399+2737$ & HDS3083AC & 2011.830 & 1 & 3.356 & 0.017 & $2.5^{*}$ & 0.3 & & & & & \\
\hline $21399+3931$ & A1445 & 2012.868 & 1 & 1.428 & 0.007 & 101.5 & 0.3 & 4.19 & & & & \\
\hline $21399+3931$ & A1445 & 2012.868 & 1 & 1.466 & 0.009 & 102.3 & 0.4 & & & & & \\
\hline $21401-0403$ & RST4697 & 2012.816 & 1 & 1.902 & 0.010 & $164.0^{*}$ & * 0.3 & & & & & \\
\hline $21423+0555$ & HU280 & 2011.754 & 1 & 0.178 & 0.040 & $178.1^{*}$ & $* 8.0$ & & & USN2006a & 0.01 & $-7.1^{Q}$ \\
\hline $21423+0555$ & HU280 & 2012.870 & 1 & 0.152 & 0.040 & $184.7^{*}$ & $* 8.0$ & & & USN2006a & -0.01 & $-3.1^{Q}$ \\
\hline $21424+4105$ & KUI108 & 2012.868 & 1 & 0.204 & 0.010 & 29.9 & 1.3 & & & Hrt2000c & 0.00 & 5.3 \\
\hline $21424+4105$ & KUI108 & 2011.759 & 1 & 0.165 & 0.040 & 28.4 & 8.0 & & & Hrt $2000 \mathrm{c}$ & -0.03 & -3.1 \\
\hline $21426+0233$ & HDS3092 & 2011.751 & 1 & 0.210 & 0.007 & 127.6 & 1.8 & & & Tok2021 & 0.03 & 11.3 \\
\hline $21426+1900$ & HO165 & 2012.854 & 1 & 0.757 & 0.007 & $61.2^{*}$ & 0.3 & & & & & \\
\hline $21428+1429$ & HEI288 & 2011.748 & 1 & 1.672 & 0.008 & $241.4^{*}$ & * 0.4 & & & & & \\
\hline $21429-0225$ & A180 & 2011.756 & 1 & 0.582 & 0.007 & $235.5^{*}$ & * 0.6 & & & & & \\
\hline
\end{tabular}




\begin{tabular}{|c|c|c|c|c|c|c|c|c|c|c|c|c|}
\hline WDS & Name & Epoch & Bin. & $\begin{array}{c}\rho \\
(")\end{array}$ & $\begin{array}{l}\sigma_{\rho} \\
(")\end{array}$ & $\begin{array}{c}\theta \\
\left(^{\circ}\right)\end{array}$ & $\begin{array}{l}\sigma_{\theta} \\
\left(^{\circ}\right)\end{array}$ & $\Delta m$ & Notes & Orbit & $\begin{array}{r}\Delta \rho(\mathrm{O}-\mathrm{C}) \\
(")\end{array}$ & $\begin{array}{r}\Delta \theta(\mathrm{O}-\mathrm{C}) \\
\left({ }^{\circ}\right)\end{array}$ \\
\hline $21431+4122$ & COU1978 & 2012.868 & 1 & 0.223 & 0.007 & $81.7^{*}$ & 0.6 & & & & & \\
\hline $21434+2353$ & HU374 & 2012.854 & 1 & 0.172 & 0.040 & $121.1^{*}$ & 8.0 & & & USN2002 & -0.06 & 70.3 \\
\hline $21435+2721$ & A299 & 2011.759 & 1 & 1.133 & 0.007 & $64.1^{*}$ & 0.3 & & & & & \\
\hline $21445+0607$ & HDS3096 & 2012.870 & 1 & 1.325 & 0.007 & $79.1^{*}$ & 0.4 & & & & & \\
\hline $21459+1153$ & A 1223 & 2011.754 & 1 & - & - & - & - & & NR & & & \\
\hline $21459+1153$ & A1223 & 2012.816 & 1 & - & - & - & - & & NR & & & \\
\hline $21460+1053$ & BU1305 & 2012.889 & 1 & 1.277 & 0.007 & $52.1^{*}$ & 0.3 & & & & & \\
\hline $21461+3534$ & COU1337 & 2011.740 & 1 & 0.345 & 0.024 & $110.4^{*}$ & 4.6 & & & & & \\
\hline $21465+2210$ & HDS3101 & 2012.854 & 1 & 0.156 & 0.040 & $12.5^{*}$ & 8.0 & & & & & \\
\hline $21466+1929$ & COU431 & 2012.889 & 1 & 0.466 & 0.007 & $185.0^{*}$ & 0.4 & & & & & \\
\hline $21469+0051$ & STF2825 & 2011.754 & 1 & 0.370 & 0.007 & $155.8^{*}$ & 0.6 & & & Zir2014a & -0.09 & 2.0 \\
\hline $21473+3451$ & COU1482 & 2012.868 & 1 & 0.807 & 0.007 & $55.7^{*}$ & 0.3 & & & & & \\
\hline $21477-0143$ & BAR15 & 2011.754 & 1 & 0.269 & 0.008 & $152.4^{*}$ & 1.2 & & & & & \\
\hline $21481+2100$ & HU378 & 2012.889 & 1 & 0.150 & 0.040 & 118.2 & 8.0 & & & & & \\
\hline $21489+0523$ & HU281 & 2011.756 & 1 & 1.458 & 0.007 & $146.6^{*}$ & 0.3 & & & & & \\
\hline $21495+2334$ & BU1306CD & 2012.889 & 1 & 1.993 & 0.010 & $348.1^{*}$ & 0.3 & & & & & \\
\hline $21496+3319$ & HDS3106 & 2011.740 & 1 & 0.140 & 0.040 & 139.5 & 8.0 & & & & & \\
\hline $21498+3455$ & COU1484 & 2012.868 & 1 & 0.405 & 0.007 & $349.2^{*}$ & 0.3 & & & & & \\
\hline $21501+1717$ & COU14 & 2012.854 & 1 & 0.291 & 0.007 & $50.2^{*}$ & 0.6 & & & Mut2010b & -0.03 & -0.9 \\
\hline $21504+3927$ & HO170 & 2011.726 & 1 & 0.283 & 0.007 & 75.8 & 0.5 & & & & & \\
\hline $21510+2911$ & A889 & 2011.726 & 1 & 0.156 & 0.040 & 126.1 & 8.0 & & & Hei1984b & 0.05 & 5.9 \\
\hline $21511+1022$ & COU2549 & 2012.816 & 1 & 0.119 & 0.044 & 85.7 & 8.0 & & & & & \\
\hline $21511+4209$ & COU1822 & 2012.868 & 1 & 0.165 & 0.040 & $19.0^{*}$ & 8.0 & & & & & \\
\hline $21518-0333$ & RST4699 & 2011.754 & 1 & 0.714 & 0.007 & $259.1^{*}$ & 0.3 & & & & & \\
\hline $21521+0549$ & J916 & 2012.870 & 1 & 1.749 & 0.009 & $5.0^{*}$ & 0.3 & & & & & \\
\hline $21539+2821$ & WSI8 & 2011.740 & 1 & 0.111 & 0.040 & 142.2 & 8.0 & & & HIP1997d & 0.02 & 2.5 \\
\hline $21543+1943$ & COU432BC & 2012.854 & 1 & 0.173 & 0.040 & $180.2^{*}$ & 8.0 & & & Tok2017c & 0.01 & $2.6^{Q}$ \\
\hline $21544+1621$ & HEI82 & 2012.854 & 1 & 1.556 & 0.008 & $73.1^{*}$ & 0.4 & & & & & \\
\hline $21546-0300$ & HDS3116 & 2011.754 & 1 & 0.231 & 0.011 & $143.7^{*}$ & 3.5 & & & & & \\
\hline $21548+4548$ & STF2846AB & 2012.764 & 1 & 3.595 & 0.018 & 85.3 & 0.3 & & & & & \\
\hline $21548+4548$ & COU2137 & 2012.764 & 1 & 0.207 & 0.007 & 39.8 & 0.9 & & & & & \\
\hline $21553+1842$ & HO173 & 2012.870 & 1 & 0.981 & 0.007 & $72.8^{*}$ & 0.3 & & & & & \\
\hline $21553+1842$ & HO173 & 2012.870 & 1 & 0.988 & 0.007 & 72.4 & 0.3 & 1.60 & & & & \\
\hline $21553+2850$ & COU836 & 2011.759 & 1 & 1.224 & 0.007 & $161.2^{*}$ & 0.3 & & & & & \\
\hline $21555+1053$ & BU75 & 2012.870 & 1 & 1.024 & 0.007 & $24.0^{*}$ & 0.3 & & & Hei1996a & 0.01 & 0.2 \\
\hline $21556+3849$ & A1449 & 2011.726 & 1 & 0.271 & 0.007 & $50.0^{*}$ & 0.9 & & & Hrt2014b & -0.02 & $-4.0^{Q}$ \\
\hline $21557+0715$ & STT452 & 2011.756 & 1 & 0.710 & 0.007 & $178.4^{*}$ & 0.3 & & & & & \\
\hline $21559+1536$ & BAT1 & 2012.870 & 1 & 1.106 & 0.007 & $152.8^{*}$ & 0.3 & & & & & \\
\hline $21561+2846$ & COU837 & 2012.868 & 1 & 1.186 & 0.007 & 150.1 & 0.3 & & & & & \\
\hline $21562+0935$ & $\mathrm{~A} 621$ & 2012.870 & 1 & - & - & - & - & & NR & & & \\
\hline $21566+3053$ & HDS3119 & 2011.740 & 1 & 0.553 & 0.008 & $32.7^{*}$ & 0.4 & & & & & \\
\hline $21571-0711$ & RST4700 & 2011.754 & 1 & 0.493 & 0.008 & $244.2^{*}$ & 0.9 & & & & & \\
\hline $21573+3241$ & A1226 & 2011.740 & 1 & 0.103 & 0.045 & $180.9^{*}$ & 6.1 & & & Cou1987c & -0.09 & $-3.9^{Q}$ \\
\hline $21576-0858$ & RST4094 & 2011.754 & 1 & 0.268 & 0.011 & 166.1 & 2.2 & & & & & \\
\hline $21577-0038$ & A891 & 2011.756 & 1 & 0.611 & 0.007 & $256.4^{*}$ & 0.3 & & & & & \\
\hline $21581-0329$ & STF2847 & 2012.816 & 1 & 0.715 & 0.007 & $306.6^{*}$ & 0.3 & & & & & \\
\hline $21588+2427$ & COU839 & 2012.854 & 1 & 0.316 & 0.007 & $95.9^{*}$ & 0.7 & & & & & \\
\hline $21590+1212$ & HEI410 & 2012.889 & 1 & 0.194 & 0.040 & $154.3^{*}$ & 8.0 & & & & & \\
\hline $21593+2641$ & COU1043 & 2011.726 & 1 & 0.362 & 0.007 & $18.2^{*}$ & 0.6 & & & & & \\
\hline $21593+4606$ & COU2138 & 2012.764 & 1 & 0.129 & 0.040 & 145.3 & 8.0 & & & Doc2012c & -0.01 & 8.9 \\
\hline $21594+1719$ & COU433 & 2012.889 & 1 & 0.588 & 0.007 & $120.3^{*}$ & 0.8 & & & & & \\
\hline $22012-0507$ & RST4703 & 2011.756 & 1 & 0.308 & 0.023 & $92.9^{*}$ & 1.5 & & & & & \\
\hline $22020+1058$ & HDS3126 & 2011.756 & 1 & 0.522 & 0.007 & 86.0 & 0.7 & & & & & \\
\hline $22021+4223$ & HDS3128 & 2012.726 & 1 & - & - & - & - & & NR & & & \\
\hline
\end{tabular}




\begin{tabular}{|c|c|c|c|c|c|c|c|c|c|c|c|c|}
\hline WDS & Name & Epoch & Bin. & $\begin{array}{c}\rho \\
(")\end{array}$ & $\begin{array}{l}\sigma_{\rho} \\
(")\end{array}$ & $\begin{array}{c}\theta \\
\left({ }^{\circ}\right)\end{array}$ & $\begin{array}{l}\sigma_{\theta} \\
\left(^{\circ}\right)\end{array}$ & $\Delta m$ & Notes & Orbit & $\begin{array}{r}\Delta \rho(\mathrm{O}-\mathrm{C}) \\
(")\end{array}$ & $\begin{array}{r}\Delta \theta(\mathrm{O}-\mathrm{C}) \\
\left({ }^{\circ}\right)\end{array}$ \\
\hline $22028-0045$ & RST5166 & 2011.756 & 1 & 0.591 & 0.007 & $12.3^{*}$ & 0.4 & & & & & \\
\hline $22029+1547$ & HDS3129 & 2011.827 & 1 & 0.154 & 0.040 & 158.4 & 8.0 & & & Tok2015c & 0.06 & -1.2 \\
\hline $22029+1547$ & HDS3129 & 2012.870 & 1 & - & - & - & - & & NR & & & \\
\hline $22029+3436$ & COU 445 & 2011.740 & 1 & 0.863 & 0.041 & 213.8 & 1.7 & & & & & \\
\hline $22029+3436$ & COU445 & 2011.742 & 1 & 0.883 & 0.007 & $211.9^{*}$ & $* 0.8$ & 0.20 & & & & \\
\hline $22032+2358$ & J289 & 2012.756 & 1 & 1.953 & 0.010 & $136.5^{*}$ & * 0.3 & & & & & \\
\hline $22032+2729$ & COU434 & 2011.742 & 1 & 0.931 & 0.024 & 92.2 & 0.7 & & & & & \\
\hline $22037+0515$ & HU491 & 2012.816 & 1 & 0.982 & 0.007 & $260.6^{*}$ & * 0.3 & & & & & \\
\hline $22038-0248$ & HO469 & 2011.756 & 1 & 0.477 & 0.007 & $52.1^{*}$ & 1.1 & & & & & \\
\hline $22038+0100$ & RST5482 & 2012.816 & 1 & 0.475 & 0.008 & $32.6^{*}$ & 1.6 & & & & & \\
\hline $22040+3841$ & COU1341 & 2012.726 & 1 & 1.599 & 0.008 & 91.2 & 0.3 & & & & & \\
\hline $22040+3841$ & COU1341 & 2011.827 & 1 & 1.542 & 0.008 & 90.4 & 0.3 & & & & & \\
\hline $22042+4821$ & COU2323 & 2011.759 & 1 & 1.612 & 0.008 & $22.1^{*}$ & 0.3 & & & & & \\
\hline $22042+4821$ & COU2323 & 2012.726 & 1 & 1.622 & 0.008 & $22.2^{*}$ & 0.3 & & & & & \\
\hline $22044+1339$ & STF2854 & 2011.751 & 1 & 1.638 & 0.008 & $83.3^{*}$ & 0.3 & & & & & \\
\hline $22045+1551$ & BU696 & 2012.870 & 1 & 0.156 & 0.040 & $356.8^{*}$ & $* 8.0$ & & & FMR2013d & -0.04 & 4.3 \\
\hline $22051+3923$ & YSC121 & 2012.756 & 1 & 1.125 & 0.007 & $102.4^{*}$ & $* 0.3$ & 4.58 & & & & \\
\hline $22051+3923$ & YSC121 & 2012.756 & 1 & 1.072 & 0.007 & 101.3 & 0.4 & & & & & \\
\hline $22053+4308$ & COU1826 & 2011.759 & 1 & 0.945 & 0.007 & $177.7^{*}$ & $* 0.3$ & & & & & \\
\hline $22053+4308$ & COU1826 & 2012.726 & 1 & 0.951 & 0.007 & $177.4^{*}$ & * 0.3 & & & & & \\
\hline $22055+0545$ & YSC73 & 2012.816 & 1 & - & - & - & - & & NR & & & \\
\hline $22055+0545$ & YSC73 & 2012.870 & 1 & 0.348 & 0.037 & 165.7 & 1.4 & & & & & \\
\hline $22055+0545$ & YSC73 & 2012.889 & 1 & 0.354 & 0.015 & 170.6 & 1.9 & & & & & \\
\hline $22059+4522$ & A183 & 2011.759 & 1 & 0.732 & 0.007 & $245.0^{*}$ & $* 0.3$ & & & & & \\
\hline $22075+2538$ & A308 & 2012.868 & 1 & 0.196 & 0.040 & $133.4^{*}$ & $* 8.0$ & & & Hrt2009 & 0.02 & $-0.1^{Q}$ \\
\hline $22077+2622$ & COU537 & 2011.759 & 1 & 0.238 & 0.007 & $140.3^{*}$ & * 0.5 & 0.10 & & Doc $2012 \mathrm{~g}$ & 0.00 & $-1.0^{Q}$ \\
\hline $22077+2622$ & COU537 & 2012.854 & 1 & 0.242 & 0.007 & 143.5 & 0.8 & & & Doc $2012 \mathrm{~g}$ & 0.00 & $-1.2^{Q}$ \\
\hline $22091+2259$ & HDS3148 & 2012.854 & 1 & 0.584 & 0.007 & $134.1^{*}$ & $* 4.0$ & & & & & \\
\hline $22095+0542$ & HWE61 & 2012.889 & 1 & 1.235 & 0.007 & $128.6^{*}$ & * 0.3 & & & & & \\
\hline $22099+4254$ & COU1828 & 2012.868 & 1 & 0.867 & 0.007 & $274.8^{*}$ & $* 0.3$ & & & & & \\
\hline $22100+2308$ & COU136 & 2012.854 & 1 & 0.457 & 0.007 & 20.7 & 0.6 & & & Doc2016h & -0.01 & -1.2 \\
\hline $22100+2308$ & COU136 & 2011.726 & 1 & 0.473 & 0.007 & 22.8 & 0.5 & & & Doc2016h & -0.00 & 0.1 \\
\hline $22106+3544$ & COU1188 & 2011.827 & 1 & 0.424 & 0.020 & $40.7^{*}$ & 0.3 & 0.80 & & & & \\
\hline $22106+3706$ & HDS3149 & 2011.830 & 1 & 0.180 & 0.040 & 138.7 & 8.0 & & & & & \\
\hline $22107+0755$ & WOR10 & 2011.754 & 1 & 0.785 & 0.007 & $12.5^{*}$ & 0.8 & & & Zir2014a & 0.00 & 0.6 \\
\hline $22107+0755$ & WOR10 & 2011.756 & 1 & 0.801 & 0.007 & $11.5^{*}$ & 0.3 & & & Zir2014a & 0.02 & -0.4 \\
\hline $22107+0755$ & WOR10 & 2012.816 & 1 & 0.835 & 0.007 & $10.9^{*}$ & 0.3 & & & Zir2014a & 0.03 & 0.5 \\
\hline $22109+2717$ & COU538 & 2011.830 & 1 & 0.119 & 0.040 & $134.5^{*}$ & $* 7.2$ & & & & & \\
\hline $22115+1806$ & CHR119 & 2011.756 & 1 & 0.188 & 0.040 & 147.3 & 8.0 & & & & & \\
\hline $22116+3727$ & COU1486 & 2011.827 & 1 & 0.570 & 0.007 & $34.0^{*}$ & 0.4 & 0.10 & & & & \\
\hline $22116+3727$ & COU1486 & 2011.742 & 1 & 0.571 & 0.007 & 31.6 & 1.1 & & & & & \\
\hline $22126+3013$ & HO179 & 2012.857 & 1 & 0.918 & 0.007 & $280.7^{*}$ & * 0.3 & & & & & \\
\hline $22134+3914$ & HDS3154 & 2011.742 & 1 & 0.546 & 0.007 & $321.9^{*}$ & $* 0.5$ & & & & & \\
\hline $22149+4142$ & $\mathrm{~A} 410$ & 2011.726 & 1 & 0.819 & 0.007 & $350.9^{*}$ & $* 0.3$ & & & & & \\
\hline $22158-0226$ & A2600 & 2011.756 & 1 & 1.759 & 0.009 & $16.5^{*}$ & 0.3 & & & & & \\
\hline $22163+4821$ & TDT3434 & 2012.889 & 1 & 0.885 & 0.007 & $263.2^{*}$ & * 0.3 & & & & & \\
\hline $22175+1649$ & HEI192 & 2012.870 & 1 & 0.127 & 0.040 & 166.7 & 8.0 & & & & & \\
\hline $22183-0022$ & HDS3160AC & 2011.754 & 1 & 1.312 & 0.014 & $119.9^{*}$ & * 0.3 & & & & & \\
\hline $22183-0022$ & HDS3160AC & 2012.816 & 1 & 1.300 & 0.018 & $120.3^{*}$ & ${ }^{*} 1.3$ & & & & & \\
\hline $22195+0501$ & TDT3464 & 2012.889 & 1 & 0.760 & 0.008 & $212.8^{*}$ & * 0.3 & & & & & \\
\hline $22195+2531$ & COU436 & 2011.830 & 1 & 0.372 & 0.007 & $259.9^{*}$ & * 1.3 & 0.20 & & & & \\
\hline $22196+2107$ & HU383 & 2012.870 & 1 & 0.359 & 0.007 & $28.9^{*}$ & 0.6 & & & & & \\
\hline $22196+2107$ & HU383 & 2012.870 & 1 & 0.333 & 0.007 & 29.3 & 0.3 & 0.63 & & & & \\
\hline $22202+2931$ & BU1216 & 2012.854 & 1 & 0.916 & 0.007 & $276.6^{*}$ & $* 0.3$ & & & Lin2012a & 0.04 & -1.3 \\
\hline
\end{tabular}




\begin{tabular}{|c|c|c|c|c|c|c|c|c|c|c|c|c|}
\hline WDS & Name & Epoch & Bin. & $\begin{array}{c}\rho \\
(")\end{array}$ & $\begin{array}{l}\sigma_{\rho} \\
(")\end{array}$ & $\begin{array}{c}\theta \\
\left(^{\circ}\right)\end{array}$ & $\begin{array}{l}\sigma_{\theta} \\
\left(^{\circ}\right)\end{array}$ & $\Delta m$ & Notes & Orbit & $\begin{array}{r}\Delta \rho(\mathrm{O}-\mathrm{C}) \\
(")\end{array}$ & $\begin{array}{r}\Delta \theta(\mathrm{O}-\mathrm{C}) \\
\left({ }^{\circ}\right)\end{array}$ \\
\hline $22210+3119$ & BU1217 & 2012.854 & 1 & 0.218 & 0.007 & $214.9^{*}$ & * 1.2 & & & & & \\
\hline $22223+2131$ & HU384 & 2011.742 & 1 & 0.225 & 0.017 & 136.4 & 3.1 & & & & & \\
\hline $22223+2131$ & HU384 & 2012.854 & 1 & 0.150 & 0.040 & 109.2 & 8.0 & & & & & \\
\hline $22223+2151$ & TDT3493 & 2012.889 & 1 & 1.156 & 0.007 & $289.7^{*}$ & * 0.3 & & & & & \\
\hline $22225-0727$ & A2694 & 2011.754 & 1 & 0.266 & 0.007 & $154.6^{*}$ & * 0.4 & & & & & \\
\hline $22234+1733$ & HO182 & 2012.870 & 1 & 1.455 & 0.007 & $310.0^{*}$ & * 0.3 & & & & & \\
\hline $22234+3228$ & WOR11 & 2011.726 & 1 & 1.401 & 0.007 & $60.6^{*}$ & 0.4 & & & Cve2011c & -0.02 & $1.0^{Q}$ \\
\hline $22234+3228$ & WOR11 & 2011.830 & 1 & 1.388 & 0.008 & $241.5^{*}$ & * 0.5 & & & Cve2011c & -0.03 & 1.6 \\
\hline $22237+1107$ & A630 & 2011.756 & 1 & 1.388 & 0.007 & $124.5^{*}$ & * 0.3 & & & & & \\
\hline $22237+2051$ & COU139CD & 2011.827 & 1 & 0.300 & 0.008 & $91.2^{*}$ & 1.1 & & & & & \\
\hline $22248+2233$ & HO183 & 2012.854 & 1 & 2.381 & 0.012 & $221.7^{*}$ & * 0.3 & & & Zir2003 & 0.06 & 0.4 \\
\hline $22248+2841$ & HDS3176 & 2011.742 & 1 & 0.320 & 0.015 & $329.5^{*}$ & * 0.7 & 0.40 & & & & \\
\hline $22255+4227$ & A2498 & 2011.726 & 1 & 0.614 & 0.007 & $345.5^{*}$ & * 0.3 & & & & & \\
\hline $22262+4228$ & COU1832 & 2011.726 & 1 & 1.016 & 0.007 & $156.9^{*}$ & * 0.3 & & & & & \\
\hline $22265+1925$ & HU596 & 2012.889 & 1 & 1.156 & 0.007 & 17.7 & 0.3 & & & & & \\
\hline $22273+3822$ & COU1643 & 2011.726 & 1 & 0.389 & 0.007 & $341.8^{*}$ & * 0.3 & & & & & \\
\hline $22276+0040$ & HDS3181 & 2011.754 & 1 & 1.249 & 0.007 & $238.1^{*}$ & * 0.4 & & & & & \\
\hline $22277-0253$ & RST4707 & 2011.756 & 1 & 0.248 & 0.007 & 156.3 & 1.0 & & & & & \\
\hline $22277+0431$ & BU291 & 2012.816 & 1 & 0.263 & 0.008 & $232.0^{*}$ & * 0.8 & & & & & \\
\hline $22281+1215$ & BU701 & 2012.870 & 1 & 0.968 & 0.007 & $181.5^{*}$ & * 0.3 & & & Cve2005 & 0.00 & 0.9 \\
\hline $22281+2942$ & BU1218 & 2011.759 & 1 & 1.517 & 0.008 & $52.8^{*}$ & 0.3 & 1.20 & & & & \\
\hline $22285+3343$ & COU942 & 2011.830 & 1 & 0.235 & 0.007 & $137.6^{*}$ & * 0.8 & 0.30 & & & & \\
\hline $22290-0956$ & HDS3183 & 2011.754 & 1 & 0.473 & 0.008 & $59.7^{*}$ & 1.1 & & & & & \\
\hline $22290+1420$ & HU1317 & 2012.870 & 1 & 0.443 & 0.007 & $126.8^{*}$ & * 0.4 & & & & & \\
\hline $22295-0012$ & BU76 & 2012.816 & 1 & 1.600 & 0.008 & $8.4^{*}$ & 0.3 & & & & & \\
\hline $22297+1848$ & TDT3549 & 2012.889 & 1 & 1.016 & 0.007 & $229.6^{*}$ & * 0.3 & & & & & \\
\hline $22302+2228$ & HU388 & 2012.854 & 1 & 0.524 & 0.007 & $59.8^{*}$ & 0.3 & & & Doc $2008 \mathrm{c}$ & -0.01 & -0.5 \\
\hline $22302+2259$ & COU140 & 2012.870 & 1 & 0.694 & 0.007 & $103.5^{*}$ & * 0.4 & & & & & \\
\hline $22303+0047$ & RST5485 & 2011.756 & 1 & 1.090 & 0.007 & 91.6 & 0.3 & & & & & \\
\hline $22303+0047$ & RST5485 & 2012.816 & 1 & 1.100 & 0.007 & $91.9^{*}$ & 0.3 & & & & & \\
\hline $22303+1810$ & COU331 & 2011.584 & 1 & 0.444 & 0.007 & $180.2^{*}$ & * 0.6 & & & & & \\
\hline $22307+1758$ & COU234 & 2012.854 & 1 & 0.163 & 0.040 & 186.4 & 8.0 & & & Doc2012c & -0.04 & 3.8 \\
\hline $22307+1758$ & COU234 & 2011.726 & 1 & 0.176 & 0.040 & 177.3 & 8.0 & & & Doc2012c & -0.01 & -8.3 \\
\hline $22308+0630$ & HDS3191 & 2011.584 & 1 & 1.289 & 0.007 & $147.7^{*}$ & * 0.3 & & & & & \\
\hline $22314+3617$ & COU1192 & 2011.830 & 1 & 0.793 & 0.010 & $182.5^{*}$ & * 1.0 & & & & & \\
\hline $22322+1311$ & BU1308BC & 2012.889 & 2 & 1.076 & 0.015 & 339.3 & 0.3 & 1.04 & & & & \\
\hline $22322+1311$ & BU1308A,BC & 2012.889 & 2 & 7.585 & 0.038 & $270.4^{*}$ & * 0.3 & & & & & \\
\hline $22328+1024$ & YSC122Aa,Ab & 2012.870 & 1 & 0.118 & 0.040 & 176.8 & 8.0 & & & & & \\
\hline $22328+1024$ & YSC122AB & 2012.870 & 1 & 1.263 & 0.020 & 118.5 & 0.3 & & & & & \\
\hline $22333+1203$ & HEI411 & 2012.816 & 1 & 1.511 & 0.008 & $302.1^{*}$ & * 0.3 & & & & & \\
\hline $22336+2217$ & J617 & 2012.854 & 1 & 0.706 & 0.007 & $93.2^{*}$ & 0.3 & & & & & \\
\hline $22344+2623$ & COU540 & 2012.854 & 1 & 0.322 & 0.007 & $322.0^{*}$ & * 0.5 & & & Mnt2004a & -0.01 & 3.3 \\
\hline $22344+2623$ & COU540 & 2011.726 & 1 & 0.308 & 0.013 & $320.9^{*}$ & * 1.0 & & & Mnt2004a & -0.03 & 3.3 \\
\hline $22352+1437$ & HU982 & 2012.941 & 1 & 0.573 & 0.007 & $216.6^{*}$ & * 0.3 & & & & & \\
\hline $22354+4341$ & COU2143 & 2012.868 & 1 & 0.337 & 0.007 & $108.0^{*}$ & * 0.4 & & & & & \\
\hline $22360+4515$ & COU2242 & 2012.868 & 1 & 0.814 & 0.007 & $275.1^{*}$ & * 0.3 & & & & & \\
\hline $22360+4515$ & GII55 & 2012.868 & 2 & 2.523 & 0.025 & $311.6^{*}$ & * 0.6 & & $\mathrm{ND}$ & & & \\
\hline $22369+1130$ & ROE130 & 2012.816 & 1 & 0.966 & 0.007 & $102.1^{*}$ & * 0.3 & & & & & \\
\hline $22372+2645$ & COU737 & 2011.584 & 1 & 0.173 & 0.040 & 13.1 & 8.0 & & & & & \\
\hline $22375+1607$ & HEI86 & 2012.870 & 1 & 0.265 & 0.007 & $100.8^{*}$ & * 0.6 & & & & & \\
\hline $22384+2943$ & HO480 & 2012.854 & 1 & 0.131 & 0.040 & 317.8 & 8.0 & & & FMR2014b & 0.02 & -11.8 \\
\hline $22386+2215$ & COU235 & 2012.870 & 1 & 0.415 & 0.018 & $74.4^{*}$ & 0.6 & & & & & \\
\hline $22389+1931$ & KUI113 & 2011.584 & 1 & 1.993 & 0.010 & 58.4 & 0.3 & & & & & \\
\hline $22396+2822$ & A413 & 2012.941 & 1 & 1.111 & 0.007 & $15.2^{*}$ & 0.3 & & & & & \\
\hline
\end{tabular}




\begin{tabular}{|c|c|c|c|c|c|c|c|c|c|c|c|c|}
\hline WDS & Name & Epoch & Bin. & $\begin{array}{c}\rho \\
(")\end{array}$ & $\begin{array}{l}\sigma_{\rho} \\
(")\end{array}$ & $\begin{array}{c}\theta \\
\left({ }^{\circ}\right)\end{array}$ & $\begin{array}{l}\sigma_{\theta} \\
\left(^{\circ}\right)\end{array}$ & $\Delta m$ & Notes & Orbit & $\begin{array}{r}\Delta \rho(\mathrm{O}-\mathrm{C}) \\
(")\end{array}$ & $\begin{array}{r}\Delta \theta(\mathrm{O}-\mathrm{C}) \\
\left({ }^{\circ}\right)\end{array}$ \\
\hline $22398+3641$ & COU1044 & 2011.582 & 1 & 0.531 & 0.011 & $117.7^{*}$ & * 0.6 & & & & & \\
\hline $22405+2002$ & WSI9 & 2012.870 & 1 & - & - & - & - & & NR & & & \\
\hline $22406+0632$ & HU494 & 2012.919 & 1 & 0.469 & 0.009 & $156.8^{*}$ & * 0.6 & & & Hrt2010a & -0.01 & $4.1^{Q}$ \\
\hline $22407+1634$ & J618 & 2012.941 & 1 & 1.836 & 0.010 & $229.6^{*}$ & * 0.4 & & & & & \\
\hline $22408-0333$ & KUI114 & 2011.754 & 1 & 0.116 & 0.007 & 137.2 & 2.0 & & & Doc2016d & 0.02 & 14.5 \\
\hline $22408-0333$ & KUI114 & 2012.816 & 1 & 0.174 & 0.040 & $134.3^{*}$ & $* 8.0$ & & & Doc2016d & -0.00 & 9.9 \\
\hline $22414+0443$ & BU480 & 2012.816 & 1 & 1.069 & 0.007 & $60.3^{*}$ & 0.3 & & & & & \\
\hline $22419+2126$ & STF2934 & 2012.840 & 1 & 1.373 & 0.007 & $57.0^{*}$ & 0.3 & & & Zir2013c & 0.05 & 1.9 \\
\hline $22420+1513$ & HU781 & 2012.889 & 1 & 0.128 & 0.040 & 157.7 & 8.0 & & & & & \\
\hline $22420+1513$ & HDS3222Aa,Ab & 2011.754 & 1 & - & - & - & - & & NR & & & \\
\hline $22421+2013$ & HDS3221 & 2011.742 & 1 & 0.598 & 0.007 & $88.2^{*}$ & 0.3 & & & & & \\
\hline $22424-0048$ & A1233 & 2012.816 & 1 & 0.233 & 0.007 & $197.4^{*}$ & $* 0.3$ & & & & & \\
\hline $22425+0254$ & HDS3223 & 2011.754 & 1 & 0.675 & 0.013 & 85.0 & 0.9 & & & & & \\
\hline $22426+2943$ & BU710 & 2012.941 & 1 & 0.490 & 0.010 & $63.8^{*}$ & 0.4 & & & & & \\
\hline $22428+2348$ & HU395A,BC & 2012.944 & 2 & 7.811 & 0.039 & $236.2^{*}$ & * 0.3 & & & & & \\
\hline $22429-0442$ & A310 & 2011.754 & 1 & 0.726 & 0.010 & $285.7^{*}$ & * 0.8 & & & & & \\
\hline $22430+0637$ & HU394 & 2012.816 & 1 & 0.208 & 0.007 & 133.9 & 0.6 & & & & & \\
\hline $22438+0353$ & WSI92 & 2012.816 & 1 & 0.975 & 0.007 & $300.0^{*}$ & * 0.3 & 3.72 & & & & \\
\hline $22438+0353$ & WSI92 & 2012.816 & 1 & 1.007 & 0.007 & 298.5 & 0.5 & & & & & \\
\hline $22438+2935$ & HDS3225 & 2011.742 & 1 & 0.926 & 0.008 & $1.5^{*}$ & 0.3 & & & & & \\
\hline $22447+1014$ & BAR17 & 2012.870 & 1 & 2.052 & 0.010 & $325.1^{*}$ & * 0.3 & & & & & \\
\hline $22455+3359$ & HU782 & 2011.879 & 1 & 1.972 & 0.010 & $321.5^{*}$ & * 0.4 & 1.40 & & & & \\
\hline $22456+4524$ & HDS3231 & 2012.868 & 1 & 0.942 & 0.007 & 344.1 & 0.3 & 2.00 & & & & \\
\hline $22456+4524$ & HDS3231 & 2012.868 & 1 & 0.934 & 0.007 & $343.6^{*}$ & * 0.3 & & & & & \\
\hline $22457+2924$ & HO481 & 2012.840 & 1 & 0.461 & 0.007 & $285.3^{*}$ & * 0.4 & & & & & \\
\hline $22459+3358$ & HJ969 & 2011.879 & 2 & 5.978 & 0.030 & $26.1^{*}$ & 0.3 & & & & & \\
\hline $22465+1944$ & COU333 & 2012.919 & 1 & 0.675 & 0.007 & $195.2^{*}$ & * 0.3 & & & & & \\
\hline $22474+1749$ & WSI93 & 2012.854 & 1 & 0.159 & 0.040 & 138.2 & 8.0 & & & Tok2017c & -0.08 & -1.2 \\
\hline $22474+1749$ & WSI93 & 2012.870 & 1 & 0.137 & 0.040 & 141.6 & 8.0 & & & Tok2017c & -0.10 & 2.0 \\
\hline $22479+1259$ & HU985 & 2011.584 & 1 & 0.721 & 0.007 & $135.7^{*}$ & * 0.8 & & & USN2002 & 0.05 & -3.3 \\
\hline $22485-0435$ & HDS3237 & 2011.754 & 1 & 0.249 & 0.020 & 352.7 & 1.0 & & & & & \\
\hline $22485-0435$ & HDS3237 & 2011.754 & 1 & 0.382 & 0.014 & $355.7^{*}$ & $* 0.5$ & & & & & \\
\hline $22485+3106$ & BU1146 & 2011.582 & 1 & 0.264 & 0.007 & $75.5^{*}$ & 0.4 & & & Doc2017i & 0.03 & 0.3 \\
\hline $22502+1810$ & HDS3243 & 2012.870 & 1 & 0.627 & 0.007 & 76.7 & 0.4 & & & & & \\
\hline $22502+1810$ & HDS3243 & 2012.870 & 1 & 0.604 & 0.007 & 73.5 & 0.3 & 3.19 & & & & \\
\hline $22513+2120$ & COU238 & 2012.854 & 1 & 0.773 & 0.007 & $160.6^{*}$ & $* 0.5$ & & & & & \\
\hline $22527+4347$ & COU2244 & 2011.582 & 1 & 0.247 & 0.010 & $288.2^{*}$ & * 0.7 & & & & & \\
\hline $22530+1407$ & $\mathrm{~J} 1347$ & 2012.870 & 1 & 1.281 & 0.007 & $352.0^{*}$ & * 0.3 & & & & & \\
\hline $22531+5001$ & COU2665 & 2011.759 & 1 & - & - & - & - & & NR & & & \\
\hline $22531+5001$ & COU2665 & 2012.868 & 1 & 0.330 & 0.007 & $88.7^{*}$ & 0.4 & & & & & \\
\hline $22532+4157$ & COU1990 & 2011.582 & 1 & 0.579 & 0.007 & $43.8^{*}$ & 0.4 & & & & & \\
\hline $22533+0740$ & HEI603 & 2011.584 & 1 & 0.235 & 0.021 & 163.5 & 1.9 & & & & & \\
\hline $22534-0906$ & HDS3251 & 2011.754 & 1 & 1.597 & 0.016 & $34.1^{*}$ & 0.3 & & & & & \\
\hline $22536+3756$ & RUC15 & 2011.827 & 1 & 1.631 & 0.008 & 79.2 & 0.3 & & & & & \\
\hline $22537+2558$ & HDS3254 & 2011.742 & 1 & 0.267 & 0.007 & 137.1 & 1.9 & & & & & \\
\hline $22545+1517$ & HEI193 & 2012.870 & 1 & 0.953 & 0.007 & $211.1^{*}$ & * 0.3 & & & & & \\
\hline $22546+1054$ & HDS3257 & 2012.816 & 1 & 0.131 & 0.040 & 67.3 & 8.0 & & & Tok2015c & -0.03 & -3.8 \\
\hline $22548+4908$ & COU2666 & 2011.759 & 1 & 1.038 & 0.010 & $139.7^{*}$ & * 0.4 & 0.20 & & & & \\
\hline $22553+4354$ & COU2245 & 2012.868 & 1 & 0.503 & 0.011 & $213.4^{*}$ & * 0.7 & & & & & \\
\hline $22553+4354$ & COU2245 & 2012.868 & 1 & 0.476 & 0.007 & 215.2 & 0.3 & 1.55 & & & & \\
\hline
\end{tabular}




\begin{tabular}{|c|c|c|c|c|c|c|c|c|c|c|c|c|}
\hline WDS & Name & Epoch & Bin. & $\begin{array}{c}\rho \\
(")\end{array}$ & $\begin{array}{l}\sigma_{\rho} \\
(")\end{array}$ & $\begin{array}{c}\theta \\
\left({ }^{\circ}\right)\end{array}$ & $\begin{array}{l}\sigma_{\theta} \\
\left(^{\circ}\right)\end{array}$ & $\Delta m$ & Notes & Orbit & $\begin{array}{r}\Delta \rho(\mathrm{O}-\mathrm{C}) \\
(")\end{array}$ & $\begin{array}{r}\Delta \theta(\mathrm{O}-\mathrm{C}) \\
\left({ }^{\circ}\right)\end{array}$ \\
\hline $22563+4247$ & A 416 & 2011.726 & 1 & 0.375 & 0.007 & $334.2^{*}$ & 0.3 & & & & & \\
\hline $22564+4443$ & COU2144 & 2012.868 & 1 & 0.985 & 0.007 & $156.2^{*}$ & 0.4 & & & & & \\
\hline $22570+2441$ & COU542Aa,Ab & 2011.584 & 1 & 0.251 & 0.007 & 73.7 & 2.7 & & & Doc2016b & 0.06 & 5.0 \\
\hline $22570+2441$ & COU542Aa,Ab & 2011.584 & 1 & 0.194 & 0.040 & 73.9 & 8.0 & & & Doc $2016 \mathrm{~b}$ & 0.00 & 5.0 \\
\hline $22570+2441$ & COU542Aa,Ab & 2012.854 & 1 & 0.128 & 0.043 & $85.4^{*}$ & 8.0 & & & Doc $2016 b$ & -0.04 & 3.8 \\
\hline $22571+3650$ & COU1644 & 2012.840 & 1 & 0.477 & 0.010 & $72.7^{*}$ & 0.5 & & & & & \\
\hline $22572+1403$ & $\mathrm{~J} 669$ & 2012.870 & 1 & 1.416 & 0.008 & $38.1^{*}$ & 0.3 & & & & & \\
\hline $22574+1958$ & COU334 & 2012.854 & 1 & 1.148 & 0.010 & 256.4 & 0.3 & & & & & \\
\hline $22574+1958$ & COU334 & 2012.854 & 1 & 1.122 & 0.007 & $255.7^{*}$ & 0.3 & 3.27 & & & & \\
\hline $22574+1958$ & COU334 & 2012.919 & 1 & 1.132 & 0.007 & $256.1^{*}$ & 0.3 & & & & & \\
\hline $22574+1958$ & COU334 & 2012.919 & 1 & 1.139 & 0.007 & 258.0 & 0.3 & 3.11 & & & & \\
\hline $22579+1337$ & HU989 & 2011.748 & 1 & 0.277 & 0.010 & $69.2^{*}$ & 1.5 & & & Hei1998 & 0.01 & 2.2 \\
\hline $22586+0921$ & STT536 & 2011.745 & 1 & 0.264 & 0.007 & $166.4^{*}$ & 0.9 & & & Sod1999 & -0.03 & -0.8 \\
\hline $22587+2611$ & COU543 & 2011.582 & 1 & 0.240 & 0.007 & $75.4^{*}$ & 1.0 & & & & & \\
\hline $22587+2611$ & COU543 & 2012.854 & 1 & 0.227 & 0.007 & $75.8^{*}$ & 1.7 & & & & & \\
\hline $22588+3808$ & HDS3272 & 2011.830 & 1 & 0.190 & 0.040 & 154.0 & 8.0 & & & & & \\
\hline $22592+1144$ & STT483 & 2011.745 & 1 & 0.462 & 0.007 & $4.6^{*}$ & 0.3 & & & Alz2007 & 0.01 & -4.9 \\
\hline $22595+4355$ & COU2146AC & 2012.868 & 1 & 3.645 & 0.018 & 145.0 & 0.3 & 5.79 & & & & \\
\hline $22595+4355$ & COU2146AC & 2012.868 & 1 & 3.642 & 0.018 & 145.1 & 0.3 & & & & & \\
\hline $22598-0226$ & HDS3274 & 2011.754 & 1 & 0.711 & 0.015 & $226.0^{*}$ & 1.1 & & & & & \\
\hline $23006-0704$ & RST4712 & 2012.816 & 1 & 1.108 & 0.007 & $187.3^{*}$ & 0.3 & & & & & \\
\hline $23009+3522$ & HU991 & 2012.840 & 1 & 0.889 & 0.007 & $300.5^{*}$ & 0.3 & & & Sca2017d & 0.01 & -0.0 \\
\hline $23013+4430$ & COU1991 & 2011.759 & 1 & 0.494 & 0.007 & $285.9^{*}$ & 0.3 & & & & & \\
\hline $23015+3103$ & ES2362 & 2012.941 & 1 & 1.980 & 0.010 & 4.0 & 0.3 & & & & & \\
\hline $23015+3516$ & COU842 & 2011.584 & 1 & 0.686 & 0.007 & $53.2^{*}$ & 0.3 & & & & & \\
\hline $23015+3516$ & COU842 & 2011.860 & 1 & 0.694 & 0.007 & $232.5^{*}$ & 0.9 & & & & & \\
\hline $23019+3642$ & COU843 & 2012.840 & 1 & 1.044 & 0.007 & $132.2^{*}$ & 0.3 & & & & & \\
\hline $23020+4800$ & A194 & 2012.868 & 1 & 0.130 & 0.045 & $287.3^{*}$ & 8.0 & & & Doc2017e & 0.02 & 7.0 \\
\hline $23022+1832$ & HDS3279AC & 2012.854 & 1 & 1.660 & 0.008 & $163.5^{*}$ & 0.3 & 4.36 & & & & \\
\hline $23022+1832$ & HDS3279AC & 2012.854 & 1 & 1.661 & 0.008 & 163.8 & 0.3 & & & & & \\
\hline $23024+1837$ & HU398 & 2011.584 & 1 & 0.482 & 0.007 & $289.1^{*}$ & 0.3 & & & Hrt2014b & 0.02 & 1.2 \\
\hline $23024+1837$ & HU398 & 2012.870 & 1 & 0.472 & 0.007 & $289.6^{*}$ & 0.3 & & & Hrt2014b & 0.00 & 0.6 \\
\hline $23033+1129$ & GII71Aa,Ab & 2012.889 & 1 & 0.139 & 0.040 & 30.7 & 8.0 & & ND & & & \\
\hline $23033+1129$ & ROE132 & 2012.870 & 1 & 1.148 & 0.022 & $71.2^{*}$ & 2.3 & & & & & \\
\hline $23033+1129$ & ROE132 & 2012.870 & 2 & 1.146 & 0.021 & 70.5 & 2.3 & 2.28 & & & & \\
\hline $23033+1129$ & ROE132 & 2012.873 & 1 & 1.149 & 0.027 & 70.5 & 2.3 & & & & & \\
\hline $23033+1129$ & ROE132 & 2012.879 & 1 & 1.153 & 0.022 & $71.3^{*}$ & 2.6 & & & & & \\
\hline $23039+2512$ & COU142 & 2012.941 & 1 & 0.521 & 0.007 & $188.3^{*}$ & 0.3 & & & & & \\
\hline $23039+3510$ & ES2134 & 2012.840 & 1 & 1.528 & 0.008 & $120.5^{*}$ & 0.3 & & & & & \\
\hline $23043+1054$ & SKF246 & 2011.879 & 1 & 1.948 & 0.024 & $117.9^{*}$ & 0.3 & & & & & \\
\hline $23052-0742$ & A417 & 2011.751 & 1 & 0.217 & 0.007 & 60.9 & 0.7 & & & Hrt1996a & 0.01 & -0.5 \\
\hline $23052-0742$ & A 417 & 2011.754 & 1 & 0.180 & 0.064 & 57.9 & 8.2 & & & Hrt1996a & -0.03 & -3.5 \\
\hline $23060+4219$ & WOR13 & 2011.582 & 1 & 0.733 & 0.007 & $142.4^{*}$ & 0.3 & & & & & \\
\hline $23067+3302$ & COU741 & 2011.879 & 1 & 0.897 & 0.007 & $192.0^{*}$ & 0.3 & & & & & \\
\hline $23075+2108$ & MAR1Aa,Ab & 2011.879 & 1 & - & - & - & - & & NR & & & \\
\hline $23082+0502$ & A1899 & 2012.919 & 1 & 1.548 & 0.008 & $64.9^{*}$ & 0.3 & & & & & \\
\hline $23083-0228$ & RST5559 & 2012.816 & 1 & 0.828 & 0.007 & $153.3^{*}$ & 0.3 & & & & & \\
\hline $23088+0047$ & HEI884 & 2012.816 & 1 & 2.018 & 0.010 & $232.8^{*}$ & 0.3 & & & & & \\
\hline $23088+1058$ & $\mathrm{~A} 1238 \mathrm{AB}$ & 2011.584 & 1 & 0.255 & 0.007 & $115.3^{*}$ & 0.8 & & & Pru2014 & -0.04 & 4.6 \\
\hline
\end{tabular}




\begin{tabular}{|c|c|c|c|c|c|c|c|c|c|c|c|c|}
\hline WDS & Name & Epoch & Bin. & $\begin{array}{c}\rho \\
(")\end{array}$ & $\begin{array}{l}\sigma_{\rho} \\
(")\end{array}$ & $\begin{array}{c}\theta \\
\left({ }^{\circ}\right)\end{array}$ & $\begin{array}{l}\sigma_{\theta} \\
\left(^{\circ}\right)\end{array}$ & $\Delta m$ & Notes & Orbit & $\begin{array}{r}\Delta \rho(\mathrm{O}-\mathrm{C}) \\
(")\end{array}$ & $\begin{array}{r}\Delta \theta(\mathrm{O}-\mathrm{C}) \\
\left({ }^{\circ}\right)\end{array}$ \\
\hline $23088+1058$ & $\mathrm{~A} 1238 \mathrm{AB}$ & 2012.873 & 1 & 0.236 & 0.011 & 105.5 & 0.3 & & & Pru2014 & -0.05 & -1.1 \\
\hline $23088+1058$ & $\mathrm{~A} 1238 \mathrm{AB}$ & 2012.873 & 1 & 0.263 & 0.007 & $106.0^{*}$ & 0.3 & & & Pru2014 & -0.02 & -0.7 \\
\hline $23088+1058$ & $\mathrm{~A} 1238 \mathrm{CD}$ & 2011.748 & 1 & 1.322 & 0.011 & 301.2 & 0.3 & & & & & \\
\hline $23088+1058$ & $\mathrm{~A} 1238 \mathrm{CD}$ & 2012.889 & 1 & 1.259 & 0.007 & $300.6^{*}$ & 0.3 & & & & & \\
\hline $23091+4153$ & COU1492 & 2011.582 & 1 & 0.896 & 0.007 & $257.1^{*}$ & 0.6 & & & & & \\
\hline $23095+0841$ & YSC16 & 2012.873 & 1 & 0.170 & 0.040 & 157.6 & 8.0 & & & & & \\
\hline $23102+3540$ & COU1198 & 2011.584 & 1 & 0.517 & 0.007 & $258.1^{*}$ & 0.5 & & & & & \\
\hline $23106+0746$ & HDS3300 & 2012.889 & 1 & 0.988 & 0.007 & $332.1^{*}$ & 0.3 & & & & & \\
\hline $23107+0947$ & HDS3302 & 2011.582 & 1 & 0.324 & 0.007 & $337.1^{*}$ & 1.9 & & & & & \\
\hline $23107+2631$ & BU852BC & 2012.941 & 1 & 1.277 & 0.013 & $320.6^{*}$ & 0.3 & & & & & \\
\hline $23107+2631$ & HDS3301 & 2012.941 & 1 & 0.770 & 0.007 & 159.8 & 0.5 & & & & & \\
\hline $23107+2631$ & HDS3301 & 2012.941 & 1 & 0.771 & 0.007 & 158.6 & 0.3 & 2.94 & & & & \\
\hline $23110+3817$ & COU1199AB & 2011.584 & 1 & 1.877 & 0.009 & 114.3 & 0.4 & & & & & \\
\hline $23110+3817$ & COU1199BC & 2011.584 & 1 & 0.853 & 0.007 & 65.4 & 0.3 & 1.63 & ND & & & \\
\hline $23126+0241$ & A2298AB & 2011.745 & 1 & - & - & - & - & & NR & & & \\
\hline $23135-0854$ & A418 & 2011.754 & 1 & 0.759 & 0.007 & $36.7^{*}$ & 0.5 & & & & & \\
\hline $23137-0618$ & RST4718 & 2012.816 & 1 & 0.753 & 0.007 & $178.9^{*}$ & 0.6 & & & & & \\
\hline $23137+0212$ & A2299 & 2011.745 & 1 & 1.246 & 0.007 & $75.1^{*}$ & 0.3 & & & & & \\
\hline $23138+4348$ & COU1992 & 2012.868 & 1 & 0.394 & 0.007 & $144.1^{*}$ & 0.3 & & & & & \\
\hline $23141-0238$ & BU714 & 2011.751 & 1 & 0.247 & 0.027 & $105.9^{*}$ & 2.0 & & & & & \\
\hline $23141+1010$ & HDS3308 & 2012.889 & 1 & 0.128 & 0.040 & 166.2 & 8.0 & & & & & \\
\hline $23144+0739$ & A1900 & 2012.873 & 1 & 1.155 & 0.007 & $239.9^{*}$ & ${ }^{*} 0.3$ & & & & & \\
\hline $23146+2555$ & COU335 & 2011.830 & 1 & 0.235 & 0.007 & 165.3 & 0.8 & & & & & \\
\hline $23159-0905$ & BU1220BC & 2011.754 & 1 & 0.597 & 0.007 & $284.2^{*}$ & 0.3 & & & Zir2007 & 0.02 & $-0.1^{Q}$ \\
\hline $23159+1430$ & $\mathrm{~J} 581$ & 2012.873 & 1 & 1.255 & 0.007 & $16.2^{*}$ & 0.3 & & & & & \\
\hline $23167+1937$ & HDS3316 & 2012.870 & 0 & 0.256 & 0.013 & 214.3 & 1.8 & & & Bag2006 & -0.03 & 1.8 \\
\hline $23167+1937$ & HDS3316 & 2012.873 & 1 & 0.308 & 0.007 & $212.0^{*}$ & ${ }^{*} 0.3$ & & & Bag2006 & 0.02 & -0.5 \\
\hline $23167+3441$ & HDS3315 & 2011.582 & 1 & 0.280 & 0.007 & $206.7^{*}$ & 0.7 & & & Cve2014 & 0.04 & 0.9 \\
\hline $23175+1652$ & HLD171A,BC & 2011.582 & 1 & 2.081 & 0.010 & $21.8^{*}$ & 0.3 & & & & & \\
\hline $23175+1652$ & HLD171A,BC & 2011.830 & 1 & 2.185 & 0.011 & $27.8^{*}$ & 0.3 & & & & & \\
\hline $23175+1652$ & HU497AB & 2012.854 & 1 & 2.210 & 0.011 & $26.0^{*}$ & 0.3 & & & & & \\
\hline $23175+1652$ & HU497BC & 2011.582 & 1 & 0.210 & 0.017 & 71.9 & 2.3 & & & Lin2010c & -0.02 & 0.5 \\
\hline $23175+1652$ & HU497BC & 2011.830 & 1 & 0.244 & 0.017 & 64.4 & 2.3 & 0.17 & & $\operatorname{Lin} 2010 \mathrm{c}$ & 0.02 & -6.6 \\
\hline $23175+1652$ & HU497BC & 2012.854 & 1 & 0.233 & 0.040 & $72.6^{*}$ & 8.0 & 0.00 & & Lin2010c & 0.00 & -3.0 \\
\hline $23176-0131$ & BU79 & 2011.751 & 1 & 1.632 & 0.008 & $11.7^{*}$ & 0.3 & & & WSI2004b & 0.03 & 1.3 \\
\hline $23176+1818$ & HU400 & 2011.827 & 1 & 0.368 & 0.018 & $77.9^{*}$ & 1.0 & & & Sca2002d & 0.00 & -2.9 \\
\hline $23176+1818$ & HU400 & 2012.854 & 1 & 0.385 & 0.008 & $74.3^{*}$ & 0.3 & & & Sca2002d & 0.01 & -4.8 \\
\hline $23178-0534$ & A419 & 2012.816 & 1 & 0.914 & 0.007 & $237.4^{*}$ & 0.3 & & & & & \\
\hline $23181-0323$ & RST4719 & 2012.816 & 1 & 2.097 & 0.010 & $221.1^{*}$ & 0.3 & & & & & \\
\hline $23189+0524$ & BU80 & 2012.873 & 1 & 0.669 & 0.007 & $241.0^{*}$ & 0.3 & & & Hei1996c & 0.04 & 3.2 \\
\hline $23191+4855$ & HDS3321 & 2011.759 & 1 & 0.601 & 0.007 & 95.8 & 0.4 & & & & & \\
\hline $23191+4855$ & HDS3321 & 2012.868 & 1 & 0.587 & 0.012 & 96.1 & 0.7 & & & & & \\
\hline $23191+4855$ & HDS3321 & 2012.868 & 1 & 0.531 & 0.007 & 95.5 & 0.3 & 2.26 & & & & \\
\hline $23195+4225$ & COU1645 & 2012.868 & 1 & 0.753 & 0.007 & $74.2^{*}$ & 0.4 & & & & & \\
\hline $23198+4243$ & COU1646 & 2011.759 & 1 & 0.179 & 0.040 & 88.8 & 8.0 & & & & & \\
\hline $23199+0807$ & HEI606 & 2012.889 & 1 & 2.159 & 0.011 & $255.1^{*}$ & ${ }^{*} 0.3$ & & & & & \\
\hline $23199+2844$ & GII36AB,C & 2011.582 & 1 & 2.496 & 0.201 & 138.4 & 4.6 & & & & & \\
\hline $23199+2844$ & GII36AB,C & 2012.854 & 1 & 2.558 & 0.013 & $139.1^{*}$ & ${ }^{*} 0.3$ & 6.01 & & & & \\
\hline $23199+2844$ & COU439 & 2011.582 & 1 & 0.165 & 0.040 & $165.2^{*}$ & 8.0 & & & Doc2017e & 0.03 & $3.0^{Q}$ \\
\hline $23200+2612$ & COU336 & 2011.827 & 1 & 1.554 & 0.008 & $359.7^{*}$ & 0.3 & & & & & \\
\hline $23204-0115$ & RST4720 & 2012.816 & 1 & 1.150 & 0.007 & $127.9^{*}$ & ${ }^{*} 0.3$ & & & & & \\
\hline $23207+4739$ & COU2668 & 2011.759 & 1 & 0.683 & 0.010 & $15.5^{*}$ & 0.8 & & & & & \\
\hline $23209+1643$ & HEI88 & 2012.854 & 1 & 0.199 & 0.040 & $180.0^{*}$ & 8.0 & & & Cve2011a & -0.03 & -0.9 \\
\hline $23209+1643$ & HEI88 & 2011.745 & 1 & 0.212 & 0.010 & 176.6 & 1.0 & & & Cve2011a & -0.01 & 0.2 \\
\hline
\end{tabular}




\begin{tabular}{|c|c|c|c|c|c|c|c|c|c|c|c|c|}
\hline WDS & Name & Epoch & Bin. & $\begin{array}{c}\rho \\
(")\end{array}$ & $\begin{array}{l}\sigma_{\rho} \\
(")\end{array}$ & $\begin{array}{c}\theta \\
\left({ }^{\circ}\right)\end{array}$ & $\begin{array}{l}\sigma_{\theta} \\
\left(^{\circ}\right)\end{array}$ & $\Delta m$ & Notes & Orbit & $\begin{array}{r}\Delta \rho(\mathrm{O}-\mathrm{C}) \\
(")\end{array}$ & $\begin{array}{r}\Delta \theta(\mathrm{O}-\mathrm{C}) \\
\left({ }^{\circ}\right)\end{array}$ \\
\hline $23213+3835$ & HDS3325 & 2011.582 & 1 & 0.142 & 0.040 & $311.0^{*}$ & * 8.0 & & & & & \\
\hline $23214+1622$ & HEI89 & 2012.873 & 1 & 1.164 & 0.007 & $67.4^{*}$ & 0.3 & & & & & \\
\hline $23216+3901$ & COU1495 & 2011.759 & 1 & 0.875 & 0.007 & $256.0^{*}$ & ${ }^{*} 0.3$ & & & & & \\
\hline $23216+3901$ & COU1495 & 2011.828 & 1 & 0.848 & 0.007 & 253.9 & 0.3 & 0.10 & & & & \\
\hline $23216+3901$ & COU1495 & 2011.830 & 1 & 0.864 & 0.007 & $254.4^{*}$ & ${ }^{*} 0.3$ & & & & & \\
\hline $23219+3149$ & BU718 & 2011.860 & 1 & 0.448 & 0.008 & $179.1^{*}$ & * 1.4 & & & & & \\
\hline $23225+0530$ & HU294 & 2012.889 & 1 & 1.868 & 0.009 & $140.6^{*}$ & * 0.3 & & & & & \\
\hline $23227+2944$ & TDT4006 & 2012.941 & 1 & 1.112 & 0.007 & $122.8^{*}$ & * 0.3 & & & & & \\
\hline $23237+4425$ & COU1994 & 2011.759 & 1 & 0.983 & 0.007 & $43.7^{*}$ & 0.5 & & & & & \\
\hline $23244+1429$ & BU719 & 2011.584 & 1 & 1.344 & 0.007 & $158.8^{*}$ & * 0.3 & & & & & \\
\hline $23249+3521$ & COU1347 & 2011.830 & 1 & 0.539 & 0.007 & $106.0^{*}$ & * 0.8 & & & & & \\
\hline $23260+2742$ & HO489 & 2011.879 & 1 & 0.520 & 0.007 & $220.3^{*}$ & * 0.5 & & & & & \\
\hline $23266+2342$ & COU338 & 2012.919 & 1 & 0.134 & 0.040 & 11.0 & 8.0 & & & & & \\
\hline $23271+1307$ & HDS3339 & 2012.816 & 1 & 0.430 & 0.011 & $233.1^{*}$ & * 1.1 & & & & & \\
\hline $23271+1307$ & HDS3339 & 2011.751 & 1 & 0.458 & 0.007 & $229.5^{*}$ & * 1.6 & & & & & \\
\hline $23272+3026$ & POP21 & 2012.941 & 1 & 0.787 & 0.007 & $210.8^{*}$ & * 0.3 & & & & & \\
\hline $23281-0228$ & RST4724 & 2012.816 & 1 & 0.121 & 0.040 & 74.8 & 8.0 & & & & & \\
\hline $23288+1512$ & HU998 & 2012.854 & 1 & 0.370 & 0.007 & $177.6^{*}$ & * 0.7 & & & & & \\
\hline $23300+1542$ & HEI90 & 2012.854 & 1 & 0.292 & 0.007 & $107.3^{*}$ & * 0.3 & & & & & \\
\hline $23300+1542$ & HEI90 & 2011.582 & 1 & 0.241 & 0.010 & $111.6^{*}$ & * 1.6 & & & & & \\
\hline $23302-0314$ & RST4725 & 2011.751 & 1 & 0.828 & 0.007 & $324.2^{*}$ & * 0.3 & & & & & \\
\hline $23302+1359$ & HU999 & 2012.889 & 1 & 1.579 & 0.008 & $142.3^{*}$ & * 0.3 & & & & & \\
\hline $23304+3050$ & BU1266 & 2011.584 & 1 & 0.161 & 0.040 & 192.6 & 8.0 & & & Msn1998c & 0.04 & -7.8 \\
\hline $23309+0929$ & STT497 & 2012.919 & 1 & 1.407 & 0.007 & $216.6^{*}$ & * 0.3 & & & & & \\
\hline $23309+3920$ & COU1348 & 2011.830 & 1 & 0.235 & 0.040 & $11.8^{*}$ & 2.6 & & & & & \\
\hline $23310+0744$ & YSC76 & 2012.816 & 1 & 0.506 & 0.013 & 162.1 & 0.7 & & & & & \\
\hline $23318+2148$ & COU243 & 2012.840 & 1 & 0.903 & 0.010 & $343.9^{*}$ & * 0.5 & & & & & \\
\hline $23322+0705$ & HU298 & 2011.582 & 1 & 0.173 & 0.040 & $93.1^{*}$ & 8.0 & & & Hrt2000c & 0.01 & 12.7 \\
\hline $23322+0705$ & HU298 & 2012.816 & 1 & 0.168 & 0.040 & $280.8^{*}$ & $* 8.0$ & & & Hrt2000c & 0.01 & $6.0^{Q}$ \\
\hline $23322+1458$ & HDS3353 & 2011.751 & 1 & 0.211 & 0.007 & $88.3^{*}$ & 8.0 & & & & & \\
\hline $23322+1458$ & HDS3353 & 2011.879 & 1 & 0.200 & 0.010 & $97.6^{*}$ & 9.9 & & & & & \\
\hline $23322+1458$ & HDS3353 & 2011.879 & 1 & 0.211 & 0.017 & 107.6 & 8.9 & & & & & \\
\hline $23322+1942$ & COU340 & 2012.854 & 1 & 0.282 & 0.007 & $56.0^{*}$ & 0.6 & & & & & \\
\hline $23324+1724$ & STF3023 & 2012.941 & 1 & 1.737 & 0.009 & $279.8^{*}$ & ${ }^{*} 0.3$ & & & & & \\
\hline $23327+3854$ & COU1349 & 2011.584 & 1 & 1.647 & 0.011 & $27.5^{*}$ & 0.3 & & & & & \\
\hline $23330+4307$ & COU2147 & 2012.868 & 1 & 1.745 & 0.009 & $341.0^{*}$ & * 0.3 & & & & & \\
\hline $23334+4251$ & HDS3356 & 2012.868 & 1 & - & - & - & - & & NR & & & \\
\hline $23336+0100$ & RST5490 & 2011.751 & 1 & 1.015 & 0.007 & $178.6^{*}$ & ${ }^{*} 0.3$ & & & & & \\
\hline $23338+1159$ & CHE504 & 2011.879 & 1 & 0.195 & 0.040 & 165.7 & 8.0 & & & & & \\
\hline $23339+2342$ & COU144 & 2011.582 & 1 & 0.323 & 0.008 & $48.4^{*}$ & 0.3 & & & & & \\
\hline $23339+4938$ & COU2670 & 2012.868 & 1 & 0.525 & 0.007 & $109.0^{*}$ & ${ }^{*} 0.3$ & & & & & \\
\hline $23342+0759$ & TDT4102AC & 2012.889 & 1 & 1.158 & 0.007 & 46.8 & 0.3 & 4.41 & $\mathrm{ND}$ & & & \\
\hline $23342+0759$ & TDT4102AC & 2012.889 & 1 & 1.158 & 0.007 & 46.2 & 0.3 & & ND & & & \\
\hline $23347+1914$ & COU341 & 2012.840 & 1 & 0.791 & 0.007 & $120.0^{*}$ & * 0.4 & & & & & \\
\hline $23361+2027$ & TDT4118 & 2012.919 & 1 & 0.604 & 0.007 & $1.4^{*}$ & 0.3 & & & & & \\
\hline $23368+2346$ & HU498 & 2012.854 & 1 & 0.685 & 0.007 & $297.1^{*}$ & * 0.3 & & & & & \\
\hline $23374+0737$ & FOX102 & 2012.873 & 1 & 0.176 & 0.040 & $68.0^{*}$ & 8.0 & & & Tok2015c & -0.00 & -4.4 \\
\hline $23374+0737$ & FOX102 & 2011.584 & 1 & 0.179 & 0.040 & 68.9 & 8.0 & & & Tok2015c & 0.00 & 3.5 \\
\hline $23379+2510$ & COU441 & 2012.941 & 1 & 0.694 & 0.007 & $11.8^{*}$ & 0.4 & & & & & \\
\hline $23380+1253$ & A1241 & 2011.584 & 1 & 0.641 & 0.007 & $11.0^{*}$ & 0.7 & & & & & \\
\hline $23382+3250$ & HU792 & 2011.879 & 1 & 0.197 & 0.040 & $131.5^{*}$ & * 8.0 & & & & & \\
\hline
\end{tabular}




\begin{tabular}{|c|c|c|c|c|c|c|c|c|c|c|c|c|}
\hline WDS & Name & Epoch & Bin. & $\begin{array}{c}\rho \\
(")\end{array}$ & $\begin{array}{l}\sigma_{\rho} \\
(")\end{array}$ & $\begin{array}{c}\theta \\
\left({ }^{\circ}\right)\end{array}$ & $\begin{array}{l}\sigma_{\theta} \\
\left({ }^{\circ}\right)\end{array}$ & $\Delta m$ & Notes & Orbit & $\begin{array}{r}\Delta \rho(\mathrm{O}-\mathrm{C}) \\
(")\end{array}$ & $\begin{array}{r}\Delta \theta(\mathrm{O}-\mathrm{C}) \\
\left({ }^{\circ}\right)\end{array}$ \\
\hline $23386+1959$ & COU342 & 2012.854 & 1 & 0.885 & 0.007 & $30.9^{*}$ & 0.3 & & & & & \\
\hline $23400+4943$ & HDS3362 & 2012.868 & 1 & 0.753 & 0.007 & $33.1^{*}$ & 0.4 & & & & & \\
\hline $23400+4943$ & HDS3362 & 2012.868 & 1 & 0.778 & 0.007 & 33.5 & 0.3 & 3.13 & & & & \\
\hline $23401+1258$ & HU1325 & 2011.879 & 1 & 0.859 & 0.007 & $30.5^{*}$ & 0.6 & & & Sca2003a & 0.01 & 3.3 \\
\hline $23402+0346$ & HEI608 & 2011.584 & 1 & 0.376 & 0.012 & 171.0 & 1.1 & & & & & \\
\hline $23405+2959$ & HDS3363 & 2011.828 & 1 & 0.898 & 0.010 & $235.1^{*}$ & * 0.6 & & & & & \\
\hline $23409+0759$ & BU724 & 2012.873 & 1 & 0.610 & 0.007 & $99.4^{*}$ & 0.3 & & & & & \\
\hline $23409+2022$ & HO303 & 2012.873 & 1 & 1.109 & 0.007 & $185.9^{*}$ & * 0.4 & & & Doc2011d & 0.12 & 0.3 \\
\hline $23413+3234$ & BU858AB & 2012.941 & 1 & 0.860 & 0.007 & $221.9^{*}$ & * 0.3 & & & & & \\
\hline $23413+4547$ & COU1998 & 2012.868 & 1 & 0.514 & 0.007 & $251.8^{*}$ & * 0.6 & & & & & \\
\hline $23420+2018$ & STT503 & 2012.920 & 1 & 1.035 & 0.007 & $133.4^{*}$ & * 0.3 & & & & & \\
\hline $23431+1150$ & A1242 & 2012.873 & 1 & 1.024 & 0.007 & $338.8^{*}$ & * 0.3 & & & Izm2019 & 0.03 & 1.1 \\
\hline $23435+1652$ & HEI196 & 2011.584 & 1 & 0.853 & 0.007 & $167.5^{*}$ & * 0.3 & & & & & \\
\hline $23440+2922$ & AGC14 & 2012.840 & 1 & 0.844 & 0.007 & $279.8^{*}$ & * 0.3 & & & Pop1997f & -0.04 & -0.1 \\
\hline $23445+0830$ & HDS3374 & 2011.584 & 1 & 0.660 & 0.013 & $39.4^{*}$ & 0.5 & & & & & \\
\hline $23445+0830$ & HDS3374 & 2012.870 & 0 & 0.655 & 0.007 & 41.1 & 0.3 & & & & & \\
\hline $23445+0830$ & HDS3374 & 2012.873 & 1 & 0.642 & 0.007 & 33.9 & 0.3 & 2.78 & & & & \\
\hline $23445+0830$ & HDS3374 & 2012.873 & 1 & 0.660 & 0.007 & $40.8^{*}$ & 0.3 & & & & & \\
\hline $23450+3020$ & $\mathrm{RAS} 15 \mathrm{Aa}, \mathrm{Ab}$ & 2012.854 & 1 & - & - & - & - & & NR & & & \\
\hline $23450+3020$ & $\mathrm{RAS} 15 \mathrm{Aa}, \mathrm{Ab}$ & 2011.879 & 1 & - & - & - & - & & NR & & & \\
\hline $23452+0814$ & WSI95 & 2012.873 & 1 & 1.177 & 0.007 & $196.8^{*}$ & * 0.3 & & & & & \\
\hline $23452+0814$ & WSI95 & 2012.873 & 1 & 1.189 & 0.007 & 195.3 & 0.3 & 3.83 & & & & \\
\hline $23455+0629$ & HU300 & 2012.920 & 1 & 1.272 & 0.007 & $109.4^{*}$ & * 0.3 & & & & & \\
\hline $23465+1705$ & EGB8 & 2011.879 & 1 & 1.075 & 0.007 & $85.2^{*}$ & 0.3 & 1.10 & & & & \\
\hline $23468+1227$ & TDT4187 & 2012.920 & 1 & 0.490 & 0.007 & $176.0^{*}$ & * 0.9 & & & & & \\
\hline $23470+1726$ & TDT4190 & 2012.980 & 1 & 0.551 & 0.007 & 33.2 & 1.0 & & & & & \\
\hline $23475+1729$ & TDT4195 & 2012.941 & 1 & 0.744 & 0.007 & 135.5 & 0.4 & & & & & \\
\hline $23485+3608$ & COU944 & 2011.828 & 1 & 0.196 & 0.040 & $102.6^{*}$ & $* 8.0$ & & & & & \\
\hline $23489+1109$ & HEI414 & 2011.751 & 1 & 1.368 & 0.007 & $76.6^{*}$ & 0.3 & & & & & \\
\hline $23491+1915$ & COU343 & 2012.854 & 1 & 0.151 & 0.040 & $113.7^{*}$ & $* 8.0$ & & & & & \\
\hline $23491+1915$ & COU343 & 2011.828 & 1 & 0.195 & 0.040 & $132.6^{*}$ & $* 8.0$ & 0.10 & & & & \\
\hline $23497+4159$ & COU1350 & 2012.868 & 1 & 0.361 & 0.007 & $195.0^{*}$ & * 0.8 & & & & & \\
\hline $23498+2741$ & $\mathrm{~A} 424$ & 2012.840 & 1 & 0.182 & 0.040 & $165.2^{*}$ & $* 8.0$ & & & Sca2002b & 0.02 & -0.3 \\
\hline $23502+1940$ & COU344 & 2011.828 & 1 & 0.215 & 0.008 & $148.1^{*}$ & $* 2.0$ & 0.10 & & Msn2001a & 0.08 & $10.8^{Q}$ \\
\hline $23502+1940$ & COU344 & 2012.873 & 1 & 0.218 & 0.007 & $147.5^{*}$ & * 1.2 & & & Msn2001a & 0.08 & $14.2^{Q}$ \\
\hline $23505-0205$ & RST4733 & 2011.751 & 1 & 0.856 & 0.007 & $52.0^{*}$ & 0.6 & & & & & \\
\hline $23509+4730$ & A794 & 2011.759 & 1 & 0.810 & 0.007 & $9.7^{*}$ & 0.6 & & & & & \\
\hline $23512+4433$ & YSC77 & 2012.868 & 1 & 0.874 & 0.010 & 99.2 & 0.3 & & & & & \\
\hline $23512+4433$ & YSC77 & 2012.868 & 1 & 0.883 & 0.007 & 101.4 & 0.3 & 4.41 & & & & \\
\hline $23518-0637$ & A2700 & 2011.751 & 1 & 0.232 & 0.007 & $170.6^{*}$ & * 0.5 & & & Doc $2009 \mathrm{~g}$ & 0.05 & 3.0 \\
\hline $23520+1252$ & A 1247 & 2011.751 & 1 & 0.413 & 0.010 & $257.0^{*}$ & $* 2.5$ & & & USN2002 & 0.10 & 12.2 \\
\hline $23520+1252$ & A 1247 & 2012.870 & 0 & 0.331 & 0.007 & 257.4 & 2.3 & & & USN2002 & 0.01 & 13.2 \\
\hline $23520+1252$ & A1247 & 2012.873 & 1 & 0.396 & 0.014 & $256.5^{*}$ & $* 2.3$ & & & USN2002 & 0.08 & 14.3 \\
\hline $23526+1057$ & YSC17 & 2011.879 & 1 & 0.587 & 0.007 & 172.6 & 1.1 & & & & & \\
\hline $23526+1057$ & YSC17 & 2012.873 & 1 & 0.579 & 0.007 & 173.6 & 0.3 & & & & & \\
\hline $23528+3841$ & A1496 & 2011.759 & 1 & 0.377 & 0.016 & $156.0^{*}$ & * 1.5 & & & & & \\
\hline $23528+3841$ & A1496 & 2011.830 & 1 & 0.454 & 0.007 & 153.8 & 0.3 & 1.95 & & & & \\
\hline $23528+3841$ & A1496 & 2011.830 & 1 & 0.413 & 0.010 & $153.5^{*}$ & * 0.4 & & & & & \\
\hline $23529+0533$ & TDT4249 & 2012.920 & 1 & 0.824 & 0.013 & $178.2^{*}$ & * 0.4 & & & & & \\
\hline $23545+2533$ & OCC9031 & 2011.828 & 1 & 0.105 & 0.047 & 10.2 & 7.2 & & & & & \\
\hline $23551+2023$ & YSC140 & 2012.854 & 1 & 0.160 & 0.040 & 108.3 & 8.0 & & & & & \\
\hline $23561+2520$ & $\mathrm{~A} 426$ & 2012.854 & 1 & 0.355 & 0.007 & $288.5^{*}$ & * 0.5 & & & Hrt2001b & -0.04 & -3.8 \\
\hline
\end{tabular}




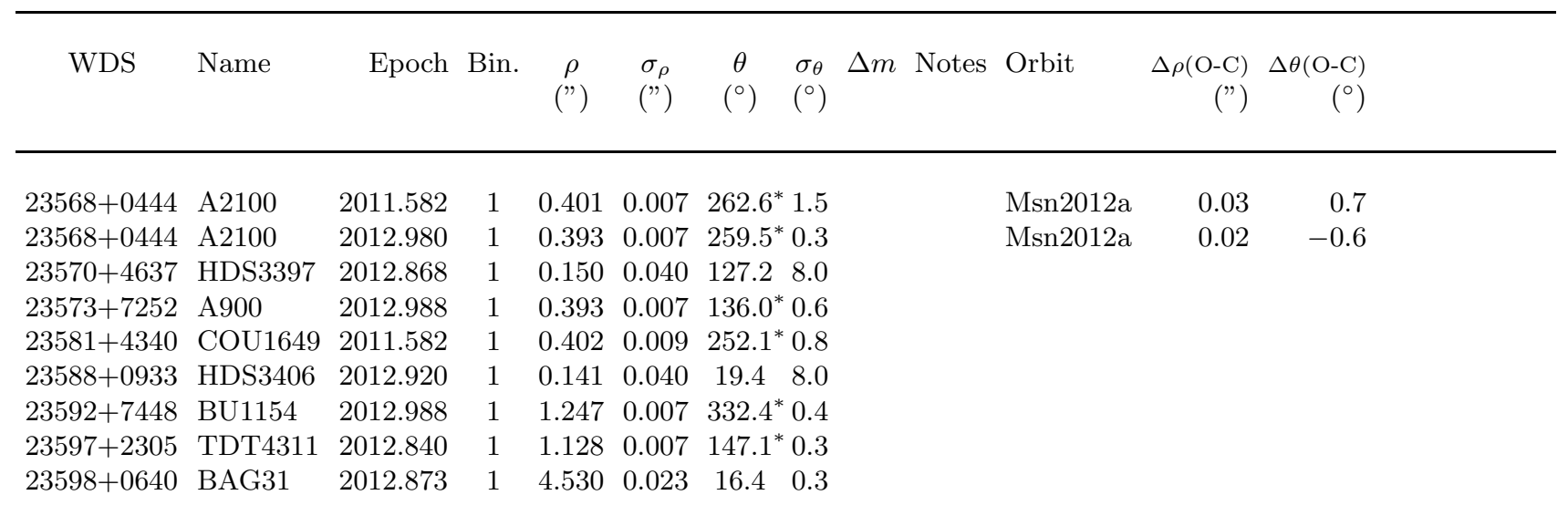

Notes: In column 7 , the superscript ${ }^{*}$ indicates that the position angle $\theta$ could be determined without the $180^{\circ}$ ambiguity.

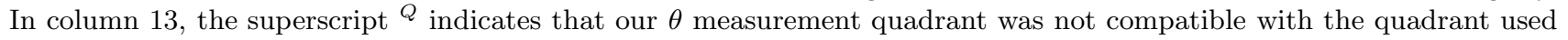
for computing the orbit. 